\title{
Arrow Spaces: A Unified Algebraic Approach to Euclidean Geometry and Inner Product Spaces
}

\author{
HUSSIN M. ALBAHBOH \\ hmalbahboh@mix.wvu.edu
}

Follow this and additional works at: https://researchrepository.wvu.edu/etd

Part of the Other Physical Sciences and Mathematics Commons

\section{Recommended Citation}

ALBAHBOH, HUSSIN M., "Arrow Spaces: A Unified Algebraic Approach to Euclidean Geometry and Inner Product Spaces" (2021). Graduate Theses, Dissertations, and Problem Reports. 8307. https://researchrepository.wvu.edu/etd/8307

This Dissertation is protected by copyright and/or related rights. It has been brought to you by the The Research Repository @ WVU with permission from the rights-holder(s). You are free to use this Dissertation in any way that is permitted by the copyright and related rights legislation that applies to your use. For other uses you must obtain permission from the rights-holder(s) directly, unless additional rights are indicated by a Creative Commons license in the record and/ or on the work itself. This Dissertation has been accepted for inclusion in WVU Graduate Theses, Dissertations, and Problem Reports collection by an authorized administrator of The Research Repository @ WVU. For more information, please contact researchrepository@mail.wvu.edu. 
Arrow Spaces: A Unified Algebraic Approach to Euclidean Geometry and Inner Product Spaces.

\author{
Hussin Albahboh \\ Dissertation submitted to the \\ Eberly College of Arts and Sciences \\ at West Virginia University \\ in partial fulfillment of the requirements \\ for the degree of
}
Doctor of Philosophy
in
Mathematics

\author{
Harry Gingold, Ph.D Co-Chair \\ Jocelyn Quaintance, Ph.D Co-Chair \\ Dening Li, Ph.D \\ Harumi Hattori, Ph.D \\ Jerzy Wojciechowski, Ph.D
}

Department of Mathematics

Morgantown, WV

2021

Keywords: Axiomatic Geometry; Euclidean Geometry; Affine Geometry; Point; Arrow; Line; Plane; Angle; Arrow Space; Vectors; Pre-inner Product

Space; Inner Product Space; Complex Hilbert Space; Similarity; Trigonometry; Trigonometric Formulas; Sine Theorem; Cosine Theorem. Copyright 2021 Hussin Albahboh 


\title{
ABSTRACT \\ Arrow Spaces: A Unified Algebraic Approach to Euclidean Geometry and Inner Product Spaces.
}

\author{
Hussin Albahboh
}

Given a postulated set of points, an algebraic system of axioms is proposed for an "arrow space". An arrow is defined to be an ordered set of two points $(T, H)$, named respectively Tail and Head. The set of arrows is an arrow space. The arrow space is axiomatically endowed with an arrow space "pre-inner product" which is analogous to the inner product of an inner product vector space over $\mathbb{R}$. Using this arrow space pre-inner product, various properties of the arrow space are derived and contrasted with the properties of a vector space over $\mathbb{R}$. The axioms of a vector space and its associated inner product are derived as theorems that follow from the axioms of an arrow space since vectors are rigorously shown to be equivalence classes of arrows. With arrow space's tools, Hilbert's axioms of Euclidean plane geometry follow as theorems in arrow spaces. Applications of using an arrow space to solve geometric problems in affine geometry are provided. Examples are provided to equip complex Hilbert spaces with a structure analogous to the structure of Euclidean geometry and trigonometry. 


\section{Acknowledgements}

While working on this dissertation, I have received a great deal of support and encouragement. This journey would not be possible without the support of my family and friends, professors, and referees. I would like to express my deepest appreciation to my supervisor, Professor Harry Gingold, who made this work possible. His continuous support, patience, and expertise proved invaluable to the completion of this dissertation. I am extremely grateful to Professor Jocelyn Quaintance for reading the manuscript and providing comments and ingenious suggestions that contributed greatly to the perfection of this work.

My sincere thanks are extended to Professors Dening Li, Harumi Hattori, and Jerzy Wojciechowski, for being amazing teachers and serving in my Ph.D. committee. I would also like to acknowledge the referees of our Paper [1] for providing insightful feedback. My sincere gratitude also goes out to my academic advisers, Professors Harvey Diamond, Jessica Deshler, and Vicki Sealey for their professional guidance. Many thanks to Professor Bernard Brooks for reading chapter 3 in the manuscript and providing grammatical feedback. And to every teacher who took part in my education, I am forever grateful for the opportunities you provided me with.

Special thanks to The Libyan Ministry of High Education and Scientific Research and The Mathematics Department at West Virginia University for funding my graduate school studies.

Finally, my deeply sincere and heartfelt gratitude goes out to my family for their unwavering support throughout this journey. I am forever indebted to my parents for giving me the opportunities that made me who I am. Special thanks to my wife and children for their unparalleled words of encouragement. You always believed in me and stood by my side. I cannot forget to thank my brothers and sisters, friends, and colleagues who encouraged me every step of the way. 


\section{Contents}

0.1 Introduction $\ldots \ldots \ldots \ldots \ldots \ldots \ldots \ldots \ldots$

1 Arrow Spaces and Vector Spaces 9

1.1 Arrow Spaces . . . . . . . . . . . . . . . . . . . . . 9 9

1.2 How Arrows Differ From Vectors . . . . . . . . . . . . . . 14

1.3 Similarities Between Arrow and Vector Spaces _ . . . . . . . . 17

1.4 Lines in an Arrow Space . . . . . . . . . . . . . . . . . . . . 21

1.4.1 Existence of Line . . . . . . . . . . . . . . . . 22

1.4.2 Equivalence Relation on $\mathscr{P}_{l_{A}} \ldots \ldots \ldots \ldots \ldots$

1.4.3 Existence of Parallel Arrow in $\mathscr{P}_{l_{A}} \ldots \ldots \ldots \ldots$

2 Turning An Arrow Space into a Vector Space 41

2.1 Existence of Parallel Arrow in $\mathscr{P}_{A} \ldots \ldots \ldots \ldots \ldots$

2.2 An Equivalence Relation on Arrow Spaces . . . . . . . . . . . . . . 49

2.3 The Associated Vector Space . . . . . . . . . . . . . . . . 56

2.4 Applications of Arrow Spaces to Affine Geometry . . . . . . . . . 65

3 Euclidean Geometry in Arrow Spaces $\quad 69$

3.1 Betweenness properties In Arrow Spaces . . . . . . . . . . . . . . . 69

3.2 Angles and trigonometry in arrow spaces . . . . . . . . . . . 79

3.3 Pasch's Axiom in Arrow Spaces . . . . . . . . . . . . . . . . . . 89

3.4 Plane Separation Theorem and Convex sets in Arrow Spaces . . . . 93

3.5 Line Circle Intersection Property in Arrow Spaces . . . . . . . . . . 101

3.6 Triangles in Arrow Spaces . . . . . . . . . . . . . . . . . . . 114

3.7 Similarity of Triangles. . . . . . . . . . . . . . . . . . 120

3.8 Axioms of Congruence in Arrow Spaces . . . . . . . . . . . . . . 124

4 Trigonometry in Complex Inner Product Spaces 128

4.1 An Appropriate Definition of The Cosine of An Angle in Complex Hilbert Spaces . . . . . . . . . . . . . . . . . . . . . 128

4.2 Cosine and Sine Theorems in Complex Hilbert Spaces _. . . . . 132 
4.3 Similarity of Triangles in Complex Hilbert Spaces. . . . . . . . . . 137

4.4 Addition Formulas in Complex Hilbert Spaces . . . . . . . . . . . . 140

5 Appendices 148

.1 Appendix A: Euclid's Axioms . . . . . . . . . . . . . . . . . . . 148

.2 Appendix B: Euclid's Common Notions . . . . . . . . . . . . . . 148

.3 Appendix C: Hilbert's Axioms of Plane Geometry . . . . . . . . . 149

.4 Appendix D: Axioms of Vector Spaces and Axioms of The Real Numbers . . . . . . . . . . . . . . . . . . 150

$\begin{array}{lr}\text { Bibliography } & \mathbf{1 5 2}\end{array}$ 


\subsection{Introduction}

An arrow is a fundamental object of mathematics and physics that is manifested graphically as a line segment with a direction. Indeed, the first appearance of a an arrow heads back to about circa 62,000 years before the common era. Another fundamental concept of mathematics and physics is the vector. Vectors are abstract algebraic quantities that are often represented by arrows. In physics there are applications where vectors are forces and the arrow is a pictorial portrayal of this force acting on a point mass. We learn from Crowe [13] that in 1687 Issac Newton used the main diagonal of the parallelogram to represent the resultant of two forces, with the understanding that the addition operation of two such forces must be commutative. This leads to the widely accepted axiom that the addition of vectors is commutative. But as we will soon see, the addition of arrows is not.

Consider three "points" $A, B$, and $C$. Let an arrow be an ordered pair of "points" $(T, H)$, where $T$ stands for Tail and $H$ stands for Head. Two arrows can be added together if and only if the head of the first is equal to the tail of the second. Consider the five distinct arrows $\overrightarrow{A B}, \overrightarrow{B C}, \overrightarrow{A C}, \overrightarrow{B A}, \overrightarrow{B B}$, and $\overrightarrow{A A}$. Define the addition operation $+_{A}$ of two arrows $\overrightarrow{A B}+{ }_{A} \overrightarrow{B C}:={ }_{A} \overrightarrow{A C}$. Notice that $\overrightarrow{B C}+{ }_{A} \overrightarrow{A B} \neq \neq_{A} \overrightarrow{A C}$ since $\overrightarrow{B C}+{ }_{A} \overrightarrow{A B}$ is undefined. Thus, $+_{A}$ is not commutative. The nature of this noncommutativity is subtle since both $\overrightarrow{A B}+{ }_{A} \overrightarrow{B A}$ and $\overrightarrow{B A}+{ }_{A} \overrightarrow{A B}$ are defined with $\overrightarrow{A B}+{ }_{A} \overrightarrow{B A}={ }_{A} \overrightarrow{A A}$ and $\overrightarrow{B A}+{ }_{A} \overrightarrow{A B}={ }_{A} \overrightarrow{B B}$, yet $\overrightarrow{A A} \neq{ }_{A} \overrightarrow{B B}$. This difference in the addition operation highlights the fact that the vector, although geometrically represented by arrow, cannot and should not be construed to be the same entity as the arrow. As we eventually see in Section 2.3, the arrow is in fact a precursor of the vector since the vector represents an equivalence class of arrows.

Although $+_{A}$ is limited to certain pairs of arrows, the addition of arrows, (when well defined), clearly encodes the starting point and the ending point within its resulting summation. As an example of the meaning of this sentence, take three points $A, B$, and $C$ and form Triangle $A B C$. We describe various paths around Triangle $A B C$ via arrow addition as shown by the three paths illustrated in Figure 0.1.

$$
\begin{aligned}
& \overrightarrow{A B}+{ }_{A} \overrightarrow{B C}+{ }_{A} \overrightarrow{C A}={ }_{A} \overrightarrow{A A}, \quad \overrightarrow{B A}+{ }_{A} \overrightarrow{A C}+{ }_{A} \overrightarrow{C B}={ }_{A} \overrightarrow{B B} \\
& \overrightarrow{C B}+{ }_{A} \overrightarrow{B A}+{ }_{A} \overrightarrow{A C}={ }_{A} \overrightarrow{C C} .
\end{aligned}
$$



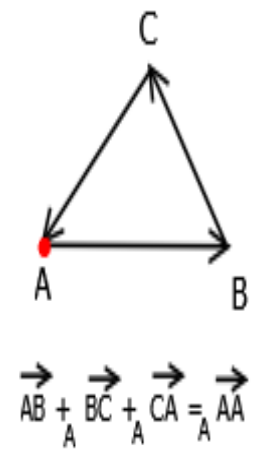
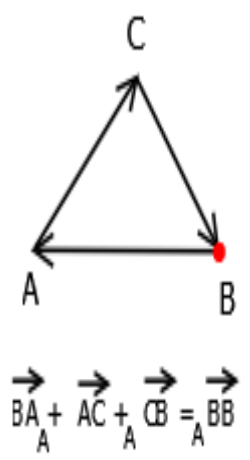
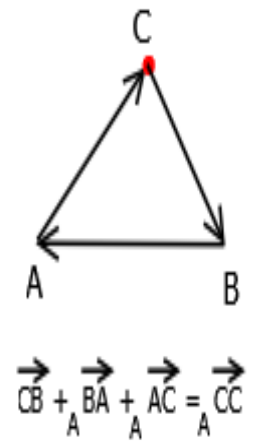

Figure 1: An illustration of three different directed paths on a triangle ABC which starts and ends at the red point.

The first begins and ends at Vertex $A$, the second begins and ends at Vertex $B$, while the third begins and ends at Vertex $C$. Let $\overrightarrow{0}$ denote the identity element of the given linear space. The equation $V+{ }_{V} W+{ }_{V} Z=\overrightarrow{0}$ is a manifestation of the three arrow relations in (1). If the points $A, B$, and $C$ are all distinct, then the vector equation $V+{ }_{V} W+{ }_{V} Z=\overrightarrow{0}$ cannot tell which of the three relations in (1) is our ultimate destination. Such information is often indispensable since the goal of many problems in geometry, calculus, and mathematical physics is to determine the final destination given an initial point of departure. Here is just a sample of three such problems.

- In elementary calculus a time dependent arrow $\overrightarrow{O P}(t)$ with tail at a coordinate system origin and head at the position of a point mass defines a definite trajectory in space. If $\overrightarrow{O P}(t)$ is differentiable at time $t$, its derivative $\frac{d \overrightarrow{O P}(t)}{d t}$ is yet another important time dependent arrow with its tail at the head of $\overrightarrow{O P}(t)$. The arrow $\frac{d \overrightarrow{O P}(t)}{d t}$ is a "tangent" arrow and it delineates the direction of a point mass along its trajectory at the time $t$ as $t$ increases; see Section 10.7 of [25].

- In another example from elementary calculus, a vector field is graphically manifested as an "arrow field', namely a collection of arrows parameterized by the point $P$ which is the tail of arrow $\overrightarrow{P F}$. The arrow $\overrightarrow{P F}$ represents the force acting on the unit point mass $P$; see Section 13.1 of [25]

- Finally, for the role of arrows in arrows in phase space analysis see Section 9.1 of [10], Section 2.8 of [11], and Sections 1.1 and 1.2 of [21].

Our approach of an arrow as an ordered set of points $(T, H)$ provides the framework to specifically determine such a final destination. 
The collection of such ordered points, which we call an arrow space, also imparts a geometric framework that solves many problems of affine geometry without resorting to a fundamental axiom of affine spaces: given a point $A$ and a vector $V$, there exists a unique point $B$ which corresponding to the "group action $+_{G A}$ " such that $B=A+{ }_{G A} V$. This axiom was advanced in the first half of the 20th century by $\mathrm{H}$. Weyl and K. O. Friedrichs, see [14]. It is the heart of the definition of an affine space, (see Definition 2.1 in [17]). The Weyl/Friedrich axiom guarantees the existence of a unique arrow $\overrightarrow{A B}$ "representing" the vector $V$ such that the tail of the arrow is the initial point $A$ and the head of the arrow is the point $B$ obtained via the group action $+_{G A}$. We remark that in the context of affine geometry, the the three relations in (1) are encapsulated by Chelsea's identity; see Section 2.3 of [17]).

One of the main goals of this dissertation is to propose a rigorous axiomatic setting of arrow spaces and their properties which is not found in either current calculus and linear algebra textbooks such as [2], [3], [4], [8], [9], [12], [25], or in the vast collection of vector analysis literature listed by Crowe [13]. Our axiomatic treatment brings out the rich structure of an arrow space that we did not encounter in the literature and uses concepts and nomenclature from set theory, algebra, and inner product spaces. In this axiomatic treatment, vectors originate naturally from arrows rather than vice versa. The fundamental nature of arrows having length and direction is being brought out in full force by the modern notion of an arrow space equipped with a pre-inner product defined on any pair of arrows. The axioms of the set of real numbers together with the supremum axiom are adopted in order guarantee that the real number line has no "holes". Hence, calculus and its theorems are readily available for use when discussing properties of the arrow space and its associated pre-inner product. The modern set of axioms of vector analysis are derived as theorems from our axioms of an arrow space by viewing vectors as equivalence classes of arrows, where two arrows are equivalent if and only if they possess same length and same direction; the concept of same direction is captured by requiring that a certain arrow pre-inner product be equal to 1 .

The notion of a vector as an equivalence class of arrows which share the same length and direction is predated by Giusto Bellavitis. According to Crowe [13], in 1835 Bellavitis publishes his first exposition on systems of equipollences. This system has some features in common with the now traditional vector analysis, as is suggested in his definition of equipollent; two straight lines are called equipollent if they are equal, parallel and directed in the same sense. His lines behave in exactly the same manner as complex numbers behave, but it is important to note that he viewed his lines as essentially geometric entities, not as geometric representations of algebraic entities. Compare also with [24].

We stress that the entities relied upon in our axiomatic treatment are algebraic. 
Geometric entities like point, line, ball, sphere, etc. are used for the "tangible" representations of algebraic entities. This is in contrast to point of view espoused by the proponents of axiomatic Euclidean geometry [20]. Thus, one may view this current work as an attempt to propose an expanded Euclidean geometry that is supplemented by arrows and vectors and has much in common with linear algebra, with inner product spaces, and with their confirmed foundation.

We would like to emphasize throughout this work that Hilbert's axioms of Euclidean Plane Geometry [[20], Chapter 2], see Appendix .3 for a full list of these axioms, hold true as theorems that follow from the axiomatic setting of arrow spaces equipped with a pre-inner product. All of the aforementioned theorems involve coordinate free proofs. One of these axioms is the so called Side-Angle-Side S.A.S. congruence of triangles. Euclid proves the S.A.S. theorem using his superposition method, which allows for moving a triangle so that it lies on top of another triangle. Evidently, Euclid did not include such an assumption in his axioms or common notions, see a full list of Euclid's axioms and common notions in Appendix .2 and .1 , respectively. However, in his set of axioms, Hilbert considers the S.A.S. to be an axiom. Hartshorne justifies Euclid's methods in a plane geometry over an ordered field by showing that there exist enough rigid motions. Thus, the existence of enough rigid motions is essentially equivalent to the statement of S.A.S, see [20] Proposition 17.1 on page 150 . In this work, we show that the S.A.S. can be proved and need not be postulated in arrow spaces. We prove it as a special case of the S.A.S similarity theorem of triangles. In fact, we prove all of the similarity theorem of triangles. The proofs of these theorems do not require Euclid's method of superposition nor the rigid motions. They rely solely on the properties of the pre-inner product of arrow spaces.

We use the $=$ symbol in three different ways: $={ }_{P}$ Equality between points, $=_{A}$ equality between arrows and $=_{V}$, and equality between vectors. We use the + operation in two different ways: addition for arrows $+_{A}$ and addition for vectors (in $\left.\mathscr{P}_{v}\right)+_{V}$. The symbol $\langle-,-\rangle_{A}$ denotes the pre inner product of arrows which will be extended to define an inner product of vectors and denoted by $\langle-,-\rangle_{V}$.

The order of work in this dissertation is as follows. In Chapter 1, Section 1.1 rigorously defines the notion of an arrow space $\mathscr{P}_{A}$. Starting with a postulated set of points $\mathscr{P}$, we define arrows as ordered pairs of points $\overrightarrow{A B}=(A, B)$. We say that two points $A, B$ are equal, denoted by $A={ }_{P} B$, if $A$ and $B$ refer to a single point. Based on this definition of equal points, equal arrows can be defined as $\overrightarrow{A B}={ }_{A} \overrightarrow{C D}$ iff $A={ }_{P} C$ and $B={ }_{P} D$. An arrow addition, denoted by $+_{A}$, is then introduced. Two arrows can be added if and only if they have a point in common, namely $\overrightarrow{A B}+{ }_{A} \overrightarrow{B C}={ }_{A} \overrightarrow{A C}$. The definition of $+_{A}$ implies if $A \neq_{P} B$, then $\overrightarrow{A B}+{ }_{A} \overrightarrow{B A} \neq_{A} \overrightarrow{B A}+{ }_{A} \overrightarrow{A B}$. Furthermore, motivated by the axioms and properties of inner product spaces, we define an arrow 
pre-inner product, denoted by $\langle-,-\rangle_{A}$, on $\mathscr{P}_{A}$. This enables us to define the measure of an arrow, denoted $\|.\|_{A}$, an arrow scalar multiplication $(t) \overrightarrow{A B}$, a line, betweenness of points, and more.

Sections 1.2 and 1.3 are devoted to making comparisons between arrows spaces and vectors spaces. Section 1.2 emphasizes the differences between arrows and vectors by focusing on how the operation of arrow addition $+_{A}$ deviates from that of vector addition. Surprisingly, arrow addition is non-commutative. A similar comparison is then applied to the respective operations of scalar multiplication. Section 1.3 focuses on the similarities between arrow spaces and vector spaces. We begin by showing that $+_{A}$, like its vector counterpart, is associative, and that both vector spaces and arrow spaces have the notion of an additive identity and an additive inverse. We also show that arrow scalar multiplication is also associative and then go on to showcase those properties of arrow scalar multiplication which have analogs in terms of vector space scalar multiplication.

In Section 1.4, we use arrow scalar multiplication to define the notion of a line in an arrow space $\mathscr{P}_{A}$ and show that given any two distinct points $A$ and $B$, there exists a unique line, denoted by $l_{A B}$, containing $A$ and $B$; see Theorem 1.11 . We then restrict our attention to the set of points that lie on a line $l$, namely the set of points $\mathscr{P}_{l}$. For $\mathscr{P}_{l_{A}}$, the arrow space associated with the line $l$, we define a relation $\mathfrak{R}_{l}$ as follows: we say that $\overrightarrow{A B} \Re_{l} \overrightarrow{C D}$ if and only if either $A={ }_{P} B$ and $C={ }_{P} D$, or

$$
\|\overrightarrow{A B}\|_{A}=\|\overrightarrow{C D}\|_{A} \text { and }\left\langle\frac{\overrightarrow{A B}}{\|\overrightarrow{A B}\|_{A}}, \frac{\overrightarrow{C D}}{\|\overrightarrow{C D}\|_{A}}\right\rangle_{A}=1
$$

From the second condition of this relation we can extract a definition of parallelism which mimics Euclid's notion of parallel lines; see Subsection 5.3. But first we prove that $\Re_{l}$ is an equivalence relation by showing that if $\overrightarrow{A B} \mathfrak{R}_{l} \overrightarrow{C D}$ and $\overrightarrow{E F} \mathfrak{R}_{l} \overrightarrow{G H}$, then $\langle\overrightarrow{A B}, \overrightarrow{E F}\rangle_{A}=\langle\overrightarrow{C D}, \overrightarrow{G H}\rangle_{A}$; see Theorem 1.12. We then exploit the definition of a line and the equivalence relation $\Re_{l}$ to prove that given any arrow $\overrightarrow{A B}$ and any point $P$ in $P_{l_{A}}$, there exists a unique parallel arrow $\overrightarrow{P K}$ (likewise a unique arrow $\overrightarrow{L P}$ ) such that $\overrightarrow{A B} \Re_{l} \overrightarrow{P K}$ (likewise $\overrightarrow{A B} \Re_{l} \overrightarrow{L P}$ ); see Theorem 1.15 .

In Chapter 2, we return to an arbitrary arrow space $\mathscr{P}_{A}$. We also prove in Section 2.1 the analog of the parallel axiom in a general arrow space. In Section 2.2, we define the relation $\mathfrak{R}$ in the context of $\mathscr{P}_{A}$; see Definition 11 . But in order to prove that $\Re$ is an equivalence relation we have to postulate Theorem 1.12 as Axiom 4. The obtained equivalence classes, denoted by $[-]$, will become vectors and turns $\mathscr{P}_{A}$ into a vector space $\mathscr{P}_{v}$. Of course we need to show that $\mathscr{P}_{v}$ satisfies all the axioms of a vector space. This means we need to convert arrow addition into vector addition, 
${ }_{V}$. Key to this conversion is the existence of a unique parallel arrow through a fixed given point, namely the analog of Theorem 1.12, which we prove for an arbitrary arrow space in Theorem 2.2. By using Theorem 2.2, we define $+_{V}$ as follows: given two vectors $[\overrightarrow{A B}]$ and $[\overrightarrow{C D}]$, and an arbitrary point $P$, let $\overrightarrow{L P}$ be the unique arrow such that $\overrightarrow{A B} \Re \overrightarrow{L P}$, and let $\overrightarrow{P K}$ be the unique arrow such that $\overrightarrow{C D} \Re \overrightarrow{P K}$. (The existence and uniqueness of the two arrows $\overrightarrow{L P}$ and $\overrightarrow{P K}$ are guaranteed by Theorem 2.2). Then,

$$
[\overrightarrow{A B}]+_{V}[\overrightarrow{C D}]=_{V}[\overrightarrow{L P}]+_{V}[\overrightarrow{P K}]=_{V}\left[\overrightarrow{L P}+{ }_{A} \overrightarrow{P K}\right]=_{V}[\overrightarrow{L K}]
$$

see Figure 1.1.
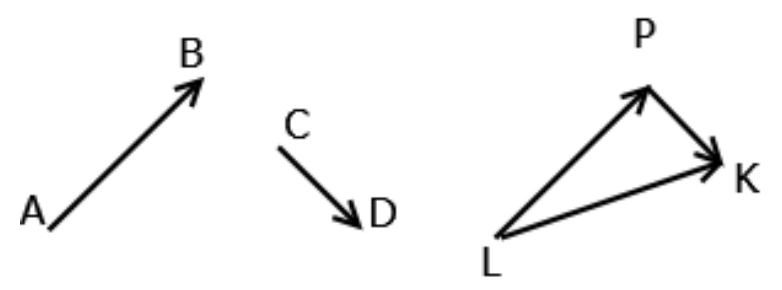

Figure 2: Adding equivalence classes of arrows.

Also arrow scalar multiplication is used to define a vector scalar multiplication as follows:

$$
t[\overrightarrow{A B}]=V[(t) \overrightarrow{A B}]
$$

In Section 2.3 we prove that the set of equivalence classes of arrows, namely $\mathscr{P}_{v}$, with the operations of vector addition and vector scalar multiplication as defined above fulfills all the axioms of vector space. Then, in Section 2.4 we demonstrate how the tools of this arrow spaces can be applicable to the field of affine geometry by solving two problems through the context of the vector space $\mathscr{P}_{v}$ associated with the arrow space $\mathscr{P}_{A}$. The first problem is to show that for a given line $l_{O G}$, for every point $P \notin l_{O G}$, there exists a unique point $W \in l_{O G}$ such that $\langle\overrightarrow{W O}, \overrightarrow{W P}\rangle_{A}=0$; see Theorem 2.12. The other application is related to the barycentric coordinates of an affine space [[16], Page 22]. We show that given a set $\left\{P_{i}\right\}_{i=1}^{n}$ of distinct points in $\mathscr{P}$ and a finite set of real numbers $\left\{\lambda_{i}\right\}_{i=1}^{n}$ such that $\sum_{i=1}^{n} \lambda_{i}=1$, for a fixed coordinate free origin $O$, there exists a unique point $M$ such that $\sum_{i=1}^{n}\left[\left(\lambda_{i}\right) \overrightarrow{O P}_{i}\right]={ }_{V}[\overrightarrow{O M}]$. Furthermore, the point $M$ is independent from the choice of the origin $O$.

In Chapter 3, we focus on Euclidean geometry in an arrow space by proving Hilbert's axioms of plane geometry that we have not treated in Chapters 1 and 2. In Section 3.1, we prove the first three axioms of betweenness, see Theorems 3.1, 3.4, and 3.3. Since the proof of the fourth axiom of betweenness (Pasch's axiom, see Theorem 3.1) requires the notion of the cosine function, Theorem 3.1 is presented in 
Section 3.3 which comes after introducing angles and the Cosine function in Section 3.2. An angle between two rays $\overrightarrow{A B}_{\infty}, \overrightarrow{A C}_{\infty}$ is the set of all points of the two rays which is not a convex set if the points $A, B$ and $C$ do not lie on the line $l_{A B}$. However, we are still able to associate with every angle a convex set of points. This is done in Section 3.4, see Remark 3.3, where we implement Theorem 3.1 (Pasch's axiom) to prove the Plane Separation Theorem in arrow space (Theorem 3.10). Then, Theorem 3.10 is employed to construct such convex set.

Section 3.5 is devoted to the Line Circle Intersection Theorem, see Theorem 3.12. In Section 3.6, we prove the laws of sines and cosines for arrow spaces, see Theorem 3.17 and 3.18, respectively. The similarity theorems of triangles in arrow spaces are included in Section 3.7, see Theorems 3.19, 3.20, and 3.21. Theorem 3.19, the S.A.S similarity Theorem, implies the S.A.S. congruence theorem if $s=1$, which represents the analog sixth axiom of congruence in arrow spaces. The other five axioms of congruence of Hilbert are proved in Section 3.8.

A list of the Axioms of an Arrow Space: Listed below is our set of axioms for an arrow space. Axioms 0 through 4 are required to set up the tools needed to construct an arrow space, derive all the axioms of vector spaces, and show that most of Hilbert's axioms of Euclidean Geometry follow as theorems in arrow spaces. Since some of Hilbert's axioms of Euclidean plane geometry, such as Pasch's axiom of betweenness and the fourth axiom of congruence, hold true only in a plane, we need to ensure the presence of a plane in an arrow space. Axiom 5 ensures the presence of planes in an arrow space. This helps us to prove the axioms (from Hilbert's axioms of Euclidean Geometry) that take place in a plane.

- Axiom 0. There exists a set of points $\mathscr{P}$.

- Axiom 1. There exists a mapping $\langle-,-\rangle_{A}: \mathscr{P}_{A} \times \mathscr{P}_{A} \longrightarrow \mathbb{R}$ such that

1. (positive definiteness)

$$
\langle\overrightarrow{A B}, \overrightarrow{A B}\rangle_{A} \geq 0 \text { and }\langle\overrightarrow{A B}, \overrightarrow{A B}\rangle_{A}=0 \text { if and only if } A={ }_{P} B
$$

2. (symmetry)

$$
\langle\overrightarrow{A B}, \overrightarrow{C D}\rangle_{A}=\langle\overrightarrow{C D}, \overrightarrow{A B}\rangle_{A}
$$

3. (arrow addition linearity)

$$
\left\langle\overrightarrow{A B}+{ }_{A} \overrightarrow{B C}, \overrightarrow{L M}\right\rangle_{A}=\langle\overrightarrow{A B}, \overrightarrow{L M}\rangle_{A}+\langle\overrightarrow{B C}, \overrightarrow{M R}\rangle_{A}
$$

4. (negation rule)

$$
\langle-\overrightarrow{A B}, \overrightarrow{C D}\rangle_{A}=(-1)\langle\overrightarrow{A B}, \overrightarrow{C D}\rangle_{A}
$$


- Axiom 2. (scalar multiplication linearity) For $\overrightarrow{A B}$ and $\overrightarrow{C D}$ any two arrows of $\mathscr{P}_{A}$ and $t \in \mathbb{R}$, we have

$$
\langle(t) \overrightarrow{A B}, \overrightarrow{C D}\rangle_{A}=t\langle\overrightarrow{A B}, \overrightarrow{C D}\rangle_{A}
$$

- Axiom 3. Let $l$ be a line containing the points $A$ and $B$. There exists a one-toone correspondence between the set of real numbers and the set of points that lie on $l$.

- Axiom 4. Given $\overrightarrow{A B}, \overrightarrow{C D}, \overrightarrow{E F}$, and $\overrightarrow{G H}$ such that $\overrightarrow{A B} \Re \overrightarrow{C D}$ and $\overrightarrow{E F} \Re \overrightarrow{G H}$, see Figure 2.5, then

$$
\langle\overrightarrow{A B}, \overrightarrow{E F}\rangle_{A}=\langle\overrightarrow{C D}, \overrightarrow{G H}\rangle_{A},
$$

where the relation $\Re$ is given in Definition 11 .

- Axiom 5. Given two distinct points $A$ and $B$, there exists a point $C$ that is different than $A$ and $B$ such that

$$
\frac{\langle\overrightarrow{A B}, \overrightarrow{A C}\rangle_{A}}{\|\overrightarrow{A B}\|_{A}\|\overrightarrow{A C}\|_{A}} \neq \pm 1
$$

Finally, Chapter 4, which includes the content of [1], serves as an example of the intimate relation between Euclidean geometry and inner product spaces. Even in a complex inner product space endowed with a general inner product, the similarity theorems, the congruence theorems of Euclid and fundamental trigonometry relations like the sine theorem, carry over from Euclidean geometry. This, with an appropriate definition of the cosine of an angle. See also [19]. 


\section{Chapter 1}

\section{Arrow Spaces and Vector Spaces}

In this chapter, we consider a set of points and establish the framework of arrow spaces. Then, we explore the similarities and differences between arrows and vectors. The rest of this chapter is dedicated to an arrow space whose underline set of points form a line.

\subsection{Arrow Spaces}

In this section, we rigorously define the notion of an arrow and an arrow space. The definition of an arrow and its associated arrow space depends on a postulated set of points.

Axiom 0. There exists a set of points $\mathscr{P}$.

We will label individual points with Roman letters and with a slight abuse of notation denote $\mathscr{P}=\{A, B, C, \ldots\}$. This labeling convention will allow us to denote equality among the elements of $\mathscr{P}$.

Definition 1. Let $\mathscr{P}=\{A, B, C, \ldots\}$ be a set of points. Let $A, B \in \mathscr{P}$. We define $A={ }_{P} B$ if and only if $A$ and $B$ refer to a single point. Otherwise, we write $A \neq{ }_{P} B$ meaning that $A$ and $B$ refer to two distinct points.

We can now define an arrow as an ordered pair of points.

Definition 2. Let $\mathscr{P}=\{A, B, C, \ldots\}$ be a set of points. Let

$$
\mathscr{P} \times \mathscr{P}=\{(A, B) \mid A, B \in \mathscr{P}\}
$$

be the Cartesian product of $\mathscr{P}$. Given any two points $A, B \in \mathscr{P}$, we define an arrow, denoted by $\overrightarrow{A B}$, to be the ordered pair $(A, B)$. The two points $A$ and $B$ are the tail and the head of the arrow $\overrightarrow{A B}$ respectively. If $A={ }_{P} B$, then $(A, B)=(A, A)$ and we denote 
the associated arrow by $\overrightarrow{A A}$. We call the set of all arrows, whose tails and heads are the points of a set $\mathscr{P}$, an arrow space and denote it by $\mathscr{P}_{A}$.

In the following definition we define equality among arrows.

Definition 3. Let $\overrightarrow{A B}, \overrightarrow{C D}$ be two arrows in $\mathscr{P}_{A}$. We put $\overrightarrow{A B}={ }_{A} \overrightarrow{C D}$ if and only if $A={ }_{P} C$ and $B={ }_{P} D$. If $A \neq{ }_{P} C$ or $B \neq{ }_{P} D$, we say that the two arrows are different and write $\overrightarrow{A B} \neq_{A} \overrightarrow{C D}$.

Next, comes a technical definition, the negation of an arrow.

Definition 4. Given the arrow $\overrightarrow{A B}$, we define $-\overrightarrow{A B}$ as $-\overrightarrow{A B}={ }_{A} \overrightarrow{B A}$; see Figure 3.1.

Note that if $A \neq_{P} B$, then $\overrightarrow{A B} \neq_{A}-\overrightarrow{A B}$.

In order to use arrows as a tool for solving various problems in affine geometry, and since arrows are actually precursors to vectors (see Section 2.2), we want to be able to manipulate them in a way that is reminiscent of the way we manipulate vectors. This means we need to define the binary operation of arrow addition and the notion of scalar multiplication acting on an arrow. Arrow addition is rather straightforward as seen by the following definition.

Definition 5. Let $\overrightarrow{A B}, \overrightarrow{B C}$ be any two arrows in $\mathscr{P}_{A}$. We define arrow addition, denoted by $+_{A}$, of $\overrightarrow{A B}$ and $\overrightarrow{B C}$ as $\overrightarrow{A B}+{ }_{A} \overrightarrow{B C}={ }_{A} \overrightarrow{A C}$.
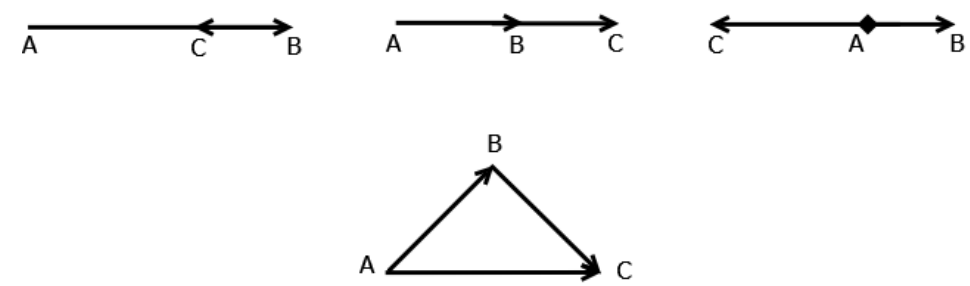

Figure 1.1: An illustration of Definition 5 where $\overrightarrow{A B}+{ }_{A} \overrightarrow{B C}={ }_{A} \overrightarrow{A C}$.

However, to define the notion scalar multiplication in an arrow space we need metric notions of length and distance, along with the Euclidean notion of an angle. These crucial notions are algebraically captured by a postulated pre-inner product, namely a symmetric, positive definite "bilinear" mapping $\langle-,-\rangle_{A}: \mathscr{P}_{A} \times \mathscr{P}_{A} \longrightarrow \mathbb{R}$. This pre-inner product will be a tool to define measure of an arrow, a line, and the betweenness notion of points that lie on a line.

Axiom 1. There exists a mapping $\langle-,-\rangle_{A}: \mathscr{P}_{A} \times \mathscr{P}_{A} \longrightarrow \mathbb{R}$ such that 
1. (positive definiteness)

$$
\langle\overrightarrow{A B}, \overrightarrow{A B}\rangle_{A} \geq 0 \text { and }\langle\overrightarrow{A B}, \overrightarrow{A B}\rangle_{A}=0 \text { if and only if } A={ }_{P} B
$$

2. (symmetry)

$$
\langle\overrightarrow{A B}, \overrightarrow{C D}\rangle_{A}=\langle\overrightarrow{C D}, \overrightarrow{A B}\rangle_{A}
$$

3. (arrow addition linearity)

$$
\left\langle\overrightarrow{A B}+{ }_{A} \overrightarrow{B C}, \overrightarrow{L M}\right\rangle_{A}=\langle\overrightarrow{A B}, \overrightarrow{L M}\rangle_{A}+\langle\overrightarrow{B C}, \overrightarrow{M R}\rangle_{A}
$$

4. (negation rule)

$$
\langle-\overrightarrow{A B}, \overrightarrow{C D}\rangle_{A}=(-1)\langle\overrightarrow{A B}, \overrightarrow{C D}\rangle_{A}
$$

Regarding Axiom 1, we make two observations which will be quite useful for proofs in the thesis. First, Equation (1.2), when combined with Equation (1.3), implies that

$$
\begin{aligned}
\left\langle\overrightarrow{A B}+{ }_{A} \overrightarrow{B C}, \overrightarrow{L M}+{ }_{A} \overrightarrow{M R}\right\rangle_{A} & =\langle\overrightarrow{A B}, \overrightarrow{L M}\rangle_{A}+\langle\overrightarrow{A B}, \overrightarrow{M R}\rangle_{A} \\
& +\langle\overrightarrow{B C}, \overrightarrow{L M}\rangle_{A}+\langle\overrightarrow{B C}, \overrightarrow{M R}\rangle_{A} .
\end{aligned}
$$

Secondly, Equation (1.2), when combined with Equation (1.4), implies that

$$
\langle\overrightarrow{A B},-\overrightarrow{C D}\rangle_{A}=(-1)\langle\overrightarrow{A B}, \overrightarrow{C D}\rangle_{A}=\langle-\overrightarrow{A B}, \overrightarrow{C D}\rangle_{A}
$$

Intuitively, we associate $\overrightarrow{A A}$ with the "zero" arrow. Thus, we would like pre-inner product of Axiom 1 to behave correctly with respect to zero, namely that $\langle\overrightarrow{A A},-\rangle=$ 0 . This is indeed the case as evidenced by the following proposition.

Proposition 1.1. For any two arrows $\overrightarrow{A A}$ and $\overrightarrow{C D}$ of $\mathscr{P}_{A}$, we have $\langle\overrightarrow{A A}, \overrightarrow{C D}\rangle_{A}=0$.

Proof : Let $\overrightarrow{A A}$ and $\overrightarrow{C D}$ be any two arrows. Since

$$
\overrightarrow{A A}={ }_{A} \overrightarrow{A D}+{ }_{A} \overrightarrow{D A} \quad \text { and } \quad \overrightarrow{C D}={ }_{A} \overrightarrow{C A}+{ }_{A} \overrightarrow{A D}
$$

Definition 4 and Equations (1.5) and (1.6) imply that

$$
\begin{aligned}
\langle\overrightarrow{A A}, \overrightarrow{C D}\rangle_{A} & =\left\langle\overrightarrow{A D}+{ }_{A} \overrightarrow{D A}, \overrightarrow{C A}+{ }_{A} \overrightarrow{A D}\right\rangle_{A} \\
& =\langle\overrightarrow{A D}, \overrightarrow{C A}\rangle_{A}+\langle\overrightarrow{A D}, \overrightarrow{A D}\rangle_{A}+\langle\overrightarrow{D A}, \overrightarrow{C A}\rangle_{A}+\langle\overrightarrow{D A}, \overrightarrow{A D}\rangle_{A} \\
& =\langle\overrightarrow{A D}, \overrightarrow{C A}\rangle_{A}+\langle\overrightarrow{A D}, \overrightarrow{A D}\rangle_{A}-\langle\overrightarrow{A D}, \overrightarrow{C A}\rangle_{A}-\langle\overrightarrow{A D}, \overrightarrow{A D}\rangle_{A}=0
\end{aligned}
$$


The pre-inner product of Axiom 1 provides a way of defining the measure (or length) of any arrow.

Definition 6. For any arrow $\overrightarrow{A B} \in \mathscr{P}_{A}$, we define a measure, denoted $\|-\|_{A}$, as follows: $\|\overrightarrow{A B}\|_{A}=\sqrt{\langle\overrightarrow{A B}, \overrightarrow{A B}\rangle_{A}}$. If $\|\overrightarrow{A B}\|_{A}=1$, then we call this arrow a unit arrow.

The following lemma is an immediate consequence of Equation (1.1) and Definition 6.

Lemma 1.1. For any arrow $\overrightarrow{A B} \in \mathscr{P}_{A}$, we have $\|\overrightarrow{A B}\|_{A}=0$ if and only if $A={ }_{P} B$.

We take advantage of the pre-inner product and the measure to define the notion of scalar multiplication in an arrow space. To avoid confusion with the negation operation of Definition 4, we will always surround the scalar multiple with parenthesis.

Definition 7. Let $\overrightarrow{A B}$ be any arrow in $\mathscr{P}_{A}$ and $t \in \mathbb{R}$. If $A={ }_{P} B$, or $t=0$, we put $(t) \overrightarrow{A B}=\overrightarrow{A A}$. If $A \neq{ }_{P} B$ and $t \neq 0$, we put $(t) \overrightarrow{A B}={ }_{A} \overrightarrow{A D}$, where $D$ is a point in $\mathscr{P}$ such that

1. $\|\overrightarrow{A D}\|_{A}=|t|\|\overrightarrow{A B}\|_{A}$

2. $\left\langle\frac{\overrightarrow{A B}}{\|\overrightarrow{A B}\|_{A}}, \frac{\overrightarrow{A D}}{\|\overrightarrow{A D}\|_{A}}\right\rangle_{A}=1$ if $t>0$, and $\left\langle\frac{\overrightarrow{A B}}{\|\overrightarrow{A B}\|_{A}}, \frac{\overrightarrow{A D}}{\|\overrightarrow{A D}\|_{A}}\right\rangle_{A}=-1$ if $t<0$.

Observe that Definition 7 only deals with the existence of the point $D$. Proposition 1.5 shows that this point is unique.

Since we now have the notion of scalar multiplication, we require that the preinner product of Axiom 1 behaves correctly with respect to this operation. In other words, we have the following axiom.

Axiom 2. (scalar multiplication linearity) For $\overrightarrow{A B}$ and $\overrightarrow{C D}$ any two arrows of $\mathscr{P}_{A}$ and $t \in \mathbb{R}$, we have

$$
\langle(t) \overrightarrow{A B}, \overrightarrow{C D}\rangle_{A}=t\langle\overrightarrow{A B}, \overrightarrow{C D}\rangle_{A} .
$$

Observe that Equation (1.7), when combined with Equation (1.2) implies that

$$
\langle(t) \overrightarrow{A B},(s) \overrightarrow{C D}\rangle_{A}=t s\langle\overrightarrow{A B}, \overrightarrow{C D}\rangle_{A},
$$

a fact that will be extremely useful for the proofs in the thesis.

The following lemma shows the relationship between length, scalar multiplication, and negation. 
Lemma 1.2. For any arrow $\overrightarrow{A B} \in \mathscr{P}_{A}$ and any $t \in \mathbb{R}$ we have

1.

$$
\|(t) \overrightarrow{A B}\|_{A}=|t|\|\overrightarrow{A B}\|_{A}
$$

2.

$$
\|\overrightarrow{A B}\|_{A}=\|\overrightarrow{B A}\|_{A}
$$

\section{Proof :}

1. Definition 6 and Equation (1.8) imply that

$$
\|(t) \overrightarrow{A B}\|_{A}^{2}=\langle(t) \overrightarrow{A B},(t) \overrightarrow{A B}\rangle_{A}=t^{2}\langle\overrightarrow{A B}, \overrightarrow{A B}\rangle_{A}=t^{2}\|\overrightarrow{A B}\|_{A}^{2}
$$

Taking the positive square root of both sides of the preceding equation gives Equation (1.9).

2. Definitions 4 and 6, along with Equation (1.6), imply that

$$
\|\overrightarrow{B A}\|_{A}^{2}=\langle\overrightarrow{B A}, \overrightarrow{B A}\rangle_{A}=\langle-\overrightarrow{A B},-\overrightarrow{A B}\rangle_{A}=(-1)(-1)\langle\overrightarrow{A B}, \overrightarrow{A B}\rangle_{A}=\|\overrightarrow{A B}\|_{A}^{2}
$$

If we take the positive square root of both sides of the preceding equation we obtain Equation (1.10).

We end this section with a theorem which relates scalar multiplication with equality of arrows.

Theorem 1.1. Let $\overrightarrow{A B}$ be an arrow in $\mathscr{P}_{A}$ such that $A \neq_{P} B$. If $(a) \overrightarrow{A B}={ }_{A}$ (b) $\overrightarrow{A B}$ for some real numbers $a$ and $b$, then we must have $a=b$.

Proof : Let $\overrightarrow{A B}$ be an arrow such that $A \neq_{P} B$ and $(a) \overrightarrow{A B}={ }_{A}(b) \overrightarrow{A B}$ for some real numbers $a$ and $b$. If $a=0$, then it follows by Definition 7 that $(a) \overrightarrow{A B}={ }_{A}(0) \overrightarrow{A B}={ }_{A}$ $\overrightarrow{A A}=(b) \overrightarrow{A B}$. Since $A \neq_{P} B$, it follows again by Definition 7 that $b=0$, which means that $a=b$. Now, suppose that $a \neq 0$ and $b \neq 0$. Then, we have by Definition 6 that

$$
\|(a) \overrightarrow{A B}\|_{A}^{2}=\langle(a) \overrightarrow{A B},(a) \overrightarrow{A B}\rangle_{A}
$$

Lemma 1.2 implies that $\|(a) \overrightarrow{A B}\|_{A}^{2}=|a|^{2}\|\overrightarrow{A B}\|_{A}^{2}$. Also, it is given that $(a) \overrightarrow{A B}={ }_{A}$ (b) $\overrightarrow{A B}$. Thus, the Equation (1.11) can be rewritten as

$$
\left.|a|^{2}\|\overrightarrow{A B}\|_{A}^{2}=\langle(a) \overrightarrow{A B},(b) \overrightarrow{A B}\rangle_{A}=a b<\overrightarrow{A B}, \overrightarrow{A B}\right\rangle_{A}
$$

where the last equality follows from by Equation (1.8). Since $\langle\overrightarrow{A B}, \overrightarrow{A B}\rangle_{A}=\|\overrightarrow{A B}\|_{A}^{2}$ (by Definition 6), the above equation implies that $|a|^{2}=a^{2}=a b$, or that $a=b$. 


\subsection{How Arrows Differ From Vectors}

For this section and the next we assume that we have an arrow space $\mathscr{P}_{A}$ and an arbitrary real vector space $V$. We want to examine the differences between $\mathscr{P}_{A}$ and $V$ and conclude that arrows are different entities than vectors. As a case in point, we show that arrow addition is restricted to certain pairs of arrows (the head of the first arrow must be the same as the tail of the second arrow) and cannot be performed on any two arbitrary arrows of $\mathscr{P}_{A}$; see Proposition 1.3. This is a fundamental difference from vector addition which is always defined for any two vectors in $V$. We also show in Proposition 1.4 that arrow addition is not commutative, a fact which draws a clear distinction between arrows and vectors. More differences between arrows and vectors will be further demonstrated.

We begin our analysis of the differences between arrows and vectors by recalling the well known fact that for any vector $v \in V$, it is always true that $(-1) v=-v$. However, this analog will not hold for arrows as witnessed by the fact that the two expressions $-\overrightarrow{A B}$ and $(-1) \overrightarrow{A B}$ in $\mathscr{P}_{A}$ are not the same.

Proposition 1.2. For any $A \neq_{P} B$, we have $-\overrightarrow{A B} \neq_{A}(-1) \overrightarrow{A B}$.
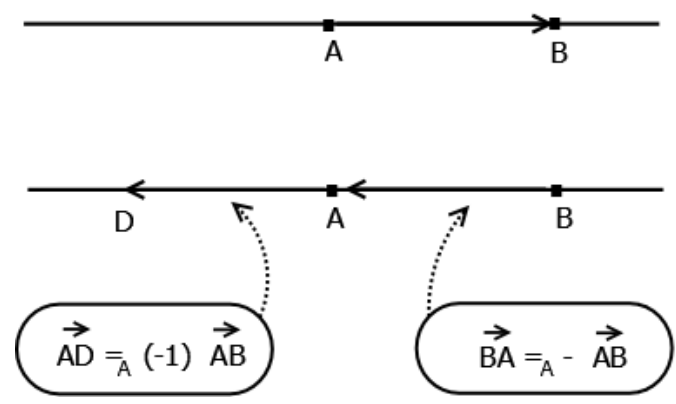

Figure 1.2: An illustration of Proposition 1.2. Note that $(-1) \overrightarrow{A B}$ and $-\overrightarrow{A B}$ have different heads and tails.

Proof : Definition 4 implies that $-\overrightarrow{A B}={ }_{A} \overrightarrow{B A}$, while Definition 7 implies that $(-1) \overrightarrow{A B}={ }_{A} \overrightarrow{A D}$, where $D \in \mathscr{P}$. Since $A \neq_{P} B$, an application of Definition 3 shows that $\overrightarrow{B A} \neq \neq_{A} \overrightarrow{A D}$, which means that $-\overrightarrow{A B} \neq_{A}(-1) \overrightarrow{A B}$.

Next, we turn our attention to the ways in which arrow addition differs from that of vector addition. The addition of arrows, as given by Definition 5, seems natural. If we have an arrow $\overrightarrow{A B}$ which has $B$ as its head, and at the same time $B$ is the tail of another arrow $\overrightarrow{B C}$, then the resultant arrow obtained from adding these two arrows 
is the arrow that has its tail as the tail of the first arrow and its head as the head of the second arrow, namely $\overrightarrow{A C}$; see Figure 1.1. But this natural definition has some drawbacks. The first drawback is that arrow addition is not defined for arbitrary pairs of arrows.

Proposition 1.3. The operation $+_{A}$ given by Definition 5 is not well defined for arbitrary elements of $\mathscr{P}_{A} \times \mathscr{P}_{A}$.

Proof : This is an immediate consequence of Definition 5 since $\overrightarrow{A B}+{ }_{A} \overrightarrow{D C}$ is not well defined if $B \neq_{P} D$.

Proposition 1.3 demonstrates a fundamental difference between arrow addition and vector addition since vector addition is defined for any element of $V \times V$. Another fundamental difference between these two operations involves commutativity. Vector addition is commutative binary operation. However, this is surprisingly not the case for arrow addition as demonstrated by the following proposition. For this reason alone, arrows and vectors should not be thought of as a single notion.

Proposition 1.4. The operation $+_{A}$ given by Definition 5 is not commutative.

Proof : If $A$ and $B$ are two distinct points of $\mathscr{P}$, then Definition 5 implies that

$$
\overrightarrow{A B}+{ }_{A} \overrightarrow{B A}={ }_{A} \overrightarrow{A A} \quad \text { and } \quad \overrightarrow{B A}+{ }_{A} \overrightarrow{A B}={ }_{A} \overrightarrow{B B}
$$

If $\overrightarrow{A B}+{ }_{A} \overrightarrow{B A}={ }_{A} \overrightarrow{B A}+{ }_{A} \overrightarrow{A B}$, then we would have $\overrightarrow{A A}={ }_{A} \overrightarrow{B B}$ which is impossible by Definition 3 as $A \neq_{P} B$. Therefore, $\overrightarrow{A B}+{ }_{A} \overrightarrow{B A} \neq_{A} \overrightarrow{B A}+{ }_{A} \overrightarrow{A B}$ implying that $+_{A}$ is noncommutative.

We now turn our attention to differences between the corresponding operations of scalar multiplication. As was the case for arrow addition, we will discover that certain notions involving scalar multiplication in an arrow space are not well defined. First we show that the property $s(v+w)=s v+s w$, where $s \in \mathbb{R}$ and $v, w \in V$, does not necessarily hold in $\mathscr{P}_{A}$.

Theorem 1.2. Let $A, B$, and $C$ be three distinct points of $\mathscr{P}$. Consider the two arrows $\overrightarrow{A B}, \overrightarrow{B C}$ in $\mathscr{P}_{A}$. For any $s \neq 1,(s)\left(\overrightarrow{A B}+{ }_{A} \overrightarrow{B C}\right)$ and $(s) \overrightarrow{A B}+{ }_{A}(s) \overrightarrow{B C}$ are not equivalent. In particular, $(s) \overrightarrow{A B}+{ }_{A}(s) \overrightarrow{B C}$ is not well defined.

Proof : First, if $s=0$, then Definitions 5 and 7 imply that

$$
(s)\left(\overrightarrow{A B}+{ }_{A} \overrightarrow{B C}\right)={ }_{A}(0) \overrightarrow{A C}={ }_{A} \overrightarrow{A A}
$$

On the other hand, let us "evaluate" expression $(s) \overrightarrow{A B}+{ }_{A}(s) \overrightarrow{B C}$. Definition 7 implies that $(0) \overrightarrow{A B}={ }_{A} \overrightarrow{A A}$ and that $(0) \overrightarrow{B C}={ }_{A} \overrightarrow{B B}$. Since $A \neq{ }_{P} B, \overrightarrow{A A}+{ }_{A} \overrightarrow{B B}$ is not well defined in the sense of Definition 5. 
Now, assume $s \neq 0,1$. It follows by Definitions 5 and 7 that

$$
\text { (s) }\left(\overrightarrow{A B}+{ }_{A} \overrightarrow{B C}\right)={ }_{A}(s) \overrightarrow{A C}={ }_{A} \overrightarrow{A M}
$$

for some points $C$ and $M$ in $\mathscr{P}$. On the other hand, Definition 7 implies that that (s) $\overrightarrow{A B}={ }_{A} \overrightarrow{A D}$ for some point $D$ in $\mathscr{P}$ with $D \neq{ }_{P} B$ (as $s \neq 1$; see Theorem 23), and that $(s) \overrightarrow{B C}={ }_{A} \overrightarrow{B E}$ for some point $E$ in $\mathscr{P}$. Thus, $(s) \overrightarrow{A B}+{ }_{A}(s) \overrightarrow{B C}={ }_{A} \overrightarrow{A D}+{ }_{A} \overrightarrow{B E}$, and by Definition 5 , as $D \neq_{P} B$, the right side is undefined.

Next, we show that the property $(s+t) v=s v+t v$, where $s, t \in \mathbb{R}$ and $v \in V$, also fails to hold in $\mathscr{P}_{A}$.

Theorem 1.3. Given an arrow $\overrightarrow{A B}$ in $\mathscr{P}_{A}$ with $A \neq \neq_{P} B$, let $s, t \in \mathbb{R}$ with $s \neq 0$. The two expressions $(s+t)(\overrightarrow{A B})$ and $(s) \overrightarrow{A B}+{ }_{A}(t) \overrightarrow{A B}$ are not equivalent. However, if $s=0$, then $(s+t)(\overrightarrow{A B})={ }_{A}(s) \overrightarrow{A B}+{ }_{A}(t) \overrightarrow{A B}$.

Proof : First assume that $s=0$. Definition 7 implies that that

$$
(t) \overrightarrow{A B}={ }_{A} \overrightarrow{A D}
$$

for some point $D$ in $\mathscr{P}$ and that

$$
(s) \overrightarrow{A B}={ }_{A} \overrightarrow{A A}
$$

Equation (1.14) implies that

$$
(s+t)(\overrightarrow{A B})={ }_{A}(t) \overrightarrow{A B}={ }_{A} \overrightarrow{A D}
$$

However, Equations (1.14) and (1.15) imply that

$$
(s) \overrightarrow{A B}+{ }_{A}(t) \overrightarrow{A B}={ }_{A} \overrightarrow{A A}+{ }_{A} \overrightarrow{A D}={ }_{A} \overrightarrow{A D}
$$

The desired equality follows by Equations (1.16) and (1.17).

Now suppose that $s \neq 0$ and let $(s) \overrightarrow{A B}={ }_{A} \overrightarrow{A C}$ and $(t) \overrightarrow{A B}={ }_{A} \overrightarrow{A D}$ for some points $C$ and $D$ in $\mathscr{P}$, with $C \neq_{P} A$. The right hand side of the expression

$$
(s) \overrightarrow{A B}+{ }_{A}(t) \overrightarrow{A B}={ }_{A} \overrightarrow{A C}+{ }_{A} \overrightarrow{A D},
$$

is undefined according to Definition 5. On the other hand, if we put $s+t=r$ for some real number $r$, then Definition 7 implies that

$$
(s+t)(\overrightarrow{A B})={ }_{A}(r) \overrightarrow{A B}={ }_{A} \overrightarrow{A E}
$$

where $E$ is a point in $\mathscr{P}$. Thus, the expression in Equation (1.19) is well defined while the right hand side of the Equation (1.18) is not well defined which makes it impossible to compare the two left hand sides of these equations.

Corollary 1.1. For any arrow $\overrightarrow{A B}$ in $\mathscr{P}_{A}$ with $A \neq_{P} B, \overrightarrow{A B}+_{A}(-1) \overrightarrow{A B}$ is not well defined. 


\subsection{Similarities Between Arrow and Vector Spaces}

So far we have investigated some axioms of vector space $V$ which do not hold in $\mathscr{P}_{A}$. In this section, we turn our attentions to those axioms of $V$ which have corresponding analogs in $\mathscr{P}_{A}$. We first show that arrow addition $+_{A}$ is indeed associative.

Theorem 1.4. Let $\overrightarrow{A B}, \overrightarrow{B C}, \overrightarrow{C D}$ be arrows in $\mathscr{P}_{A}$. Then,

$$
\left(\overrightarrow{A B}+{ }_{A} \overrightarrow{B C}\right)+{ }_{A} \overrightarrow{C D}={ }_{A} \overrightarrow{A B}+{ }_{A}\left(\overrightarrow{B C}+{ }_{A} \overrightarrow{C D}\right)
$$

that is the addition $+_{A}$ is associative.
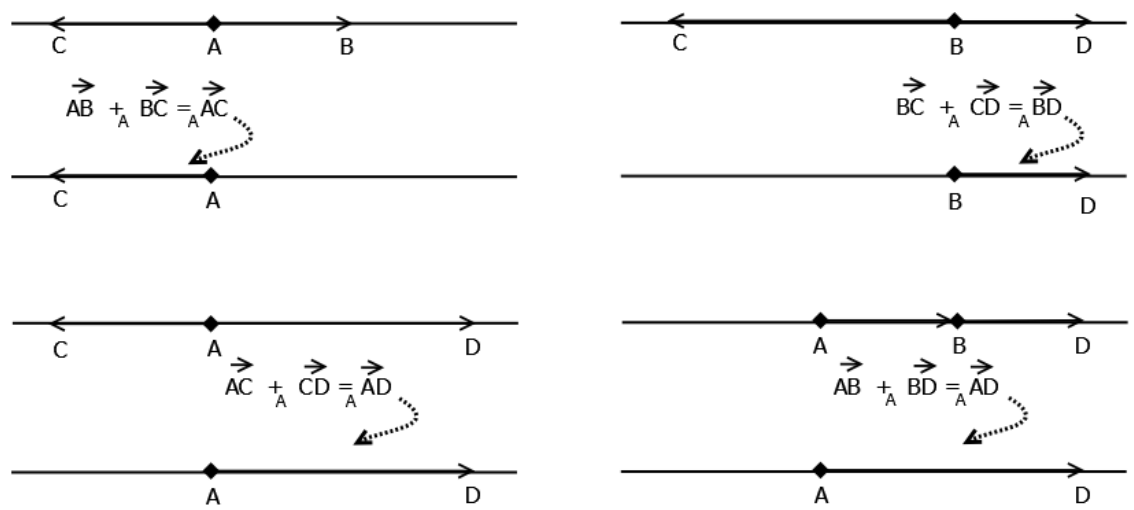

Figure 1.3: An illustration of Theorem 1.4. The left section of this figure explains $\left(\overrightarrow{A B}+{ }_{A} \overrightarrow{B C}\right)+{ }_{A} \overrightarrow{C D}$, while the right one stands for $\overrightarrow{A B}+{ }_{A}\left(\overrightarrow{B C}+{ }_{A} \overrightarrow{C D}\right)$.

Proof : Given three arrows $\overrightarrow{A B}, \overrightarrow{B C}$, and $\overrightarrow{C D}$ in $\mathscr{P}_{A}$, Definition 5 implies that

$$
\left(\overrightarrow{A B}+{ }_{A} \overrightarrow{B C}\right)+{ }_{A} \overrightarrow{C D}={ }_{A} \overrightarrow{A C}+{ }_{A} \overrightarrow{C D}={ }_{A} \overrightarrow{A D}
$$

A similar calculation shows that

$$
\overrightarrow{A B}+{ }_{A}\left(\overrightarrow{B C}+{ }_{A} \overrightarrow{C D}\right)={ }_{A} \overrightarrow{A B}+{ }_{A} \overrightarrow{B D}={ }_{A} \overrightarrow{A D}
$$

Combining the Equations (1.20) and (1.21) finishes the proof.

A similar result regarding associativity occurs for arrow scalar multiplication. But in order to prove the associativity of arrow scalar multiplication we will need the following proposition. This proposition is important in its own right since it will be crucial to proving the existence of line between any two points; see Subsection 5.1.

Proposition 1.5. Let $\overrightarrow{A B}$ and $\overrightarrow{A D}$ be two arrows of $\mathscr{P}_{A}$ such that $\|\overrightarrow{A B}\|_{A}=\|\overrightarrow{A D}\|_{A}$ and $\frac{\langle\overrightarrow{A B}, \overrightarrow{A D}\rangle_{A}}{\|\overrightarrow{A B}\|_{A}\|\overrightarrow{A D}\|_{A}}=1$. Then, $B={ }_{P} D$. 
Proof : Assume by way of contradiction, that $B \neq_{P} D$. By hypothesis we have $\|\overrightarrow{A B}\|_{A}=\|\overrightarrow{A D}\|_{A}$ and $\frac{\langle\overrightarrow{A B}, \overrightarrow{A D}\rangle_{A}}{\left\|\left.\overrightarrow{A B}\right|_{A}\right\| \overrightarrow{A D} \|_{A}}=1$, which implies that

$$
\langle\overrightarrow{A B}, \overrightarrow{A D}\rangle_{A}=\langle\overrightarrow{A D}, \overrightarrow{A D}\rangle_{A}
$$

An application of Axiom 1 and Definition 4 to Equation (1.21) yields

$$
\langle\overrightarrow{D A}, \overrightarrow{A D}\rangle_{A}+\langle\overrightarrow{A B}, \overrightarrow{A D}\rangle_{A}=\langle\overrightarrow{D B}, \overrightarrow{A D}\rangle_{A}=0
$$

Since $\overrightarrow{A D}={ }_{A}-\overrightarrow{D A}$ (see Definition 4), the preceding equation is equivalent to

$$
\langle\overrightarrow{D B}, \overrightarrow{D A}\rangle_{A}=0
$$

On the other hand, since $\|\overrightarrow{A B}\|_{A}=\|\overrightarrow{A D}\|_{A}$ we can rewrite Equation (1.21) as

$$
\langle\overrightarrow{A B}, \overrightarrow{A D}\rangle_{A}=\langle\overrightarrow{A B}, \overrightarrow{A B}\rangle_{A}
$$

Then, a similar argument shows that

$$
\langle\overrightarrow{D B}, \overrightarrow{A B}\rangle_{A}=0
$$

Adding Equations (1.22) and (1.23) and using Axiom 1 gives us

$$
\langle\overrightarrow{D B}, \overrightarrow{D A}\rangle_{A}+\langle\overrightarrow{D B}, \overrightarrow{A B}\rangle_{A}=\langle\overrightarrow{D B}, \overrightarrow{D B}\rangle_{A}=0
$$

By Definition 6, Equation (1.24) means that $\|\overrightarrow{D B}\|_{A}^{2}=0$, that is $\|\overrightarrow{D B}\|_{A}=0$. But by Lemma 1.1 , the equation $\|\overrightarrow{D B}\|_{A}=0$ implies that $D={ }_{P} B$ which contradicts our assumption that $B \neq_{P} D$. Thus, we conclude that we must have $B={ }_{P} D$.

Theorem 1.5. For any $s, t \in \mathbb{R}$ and any arrow $\overrightarrow{A B}$ in $\mathscr{P}_{A}$, $(s t) \overrightarrow{A B}={ }_{A}(s)((t) \overrightarrow{A B})$, that is the arrow scalar multiplication is associative.

Proof : If $A={ }_{P} B$ or at least one of the real numbers $s$ or $t$ is zero, then it follows directly from Definition 7 that $(s t) \overrightarrow{A B}={ }_{A}(s)((t) \overrightarrow{A B})={ }_{A} \overrightarrow{A A}$. Now, let us assume $A \neq \neq_{P} B$ and $s, t \neq 0$. Let

$$
\begin{gathered}
(t) \overrightarrow{A B}={ }_{A} \overrightarrow{A C} \\
(s)((t) \overrightarrow{A B})={ }_{A}(s) \overrightarrow{A C}={ }_{A} \overrightarrow{A D}
\end{gathered}
$$

and

$$
(s t) \overrightarrow{A B}={ }_{A} \overrightarrow{A F}
$$


for some points $C, D$, and $F$ on the line $l_{A B}$. We want to show that $\overrightarrow{A D}={ }_{A} \overrightarrow{A F}$. By Definition 7 and Equations (1.25) and (1.26) we have

$$
\|\overrightarrow{A C}\|_{A}=|t|\|\overrightarrow{A B}\|_{A}, \quad\|\overrightarrow{A D}\|_{A}=|s|\|\overrightarrow{A C}\|_{A}
$$

which implies that

$$
\|\overrightarrow{A D}\|_{A}=|s t|\|\overrightarrow{A B}\|_{A}
$$

Also, Definition 7 and Equation (1.27) imply that

$$
\|\overrightarrow{A F}\|_{A}=|s t|\|\overrightarrow{A B}\|_{A}
$$

We get from Equations (1.28) and (1.29) that

$$
\|\overrightarrow{A F}\|_{A}=\|\overrightarrow{A D}\|_{A}
$$

Next, we show that $\left\langle\frac{\overrightarrow{A D}}{\|\overrightarrow{A D}\|_{A}}, \frac{\overrightarrow{A F}}{\|\overrightarrow{A F}\|_{A}}\right\rangle_{A}=1$. By Equations (1.26), (1.27), and since $\|\overrightarrow{A F}\|_{A}=\|\overrightarrow{A D}\|_{A}=|s t|\|\overrightarrow{A B}\|_{A}$, we have

$$
\left\langle\frac{\overrightarrow{A D}}{\|\overrightarrow{A D}\|_{A}}, \frac{\overrightarrow{A F}}{\|\overrightarrow{A F}\|_{A}}\right\rangle_{A}=\left\langle\frac{s((t) \overrightarrow{A B})}{|s t|\|\overrightarrow{A B}\|_{A}}, \frac{(s t) \overrightarrow{A B}}{|s t|\|\overrightarrow{A B}\|_{A}}\right\rangle_{A}
$$

Using Equation (1.8) and Definition 6, the preceding equation simplifies to

$$
\left\langle\frac{\overrightarrow{A D}}{\|\overrightarrow{A D}\|_{A}}, \frac{\overrightarrow{A F}}{\|\overrightarrow{A F}\|_{A}}\right\rangle_{A}=\frac{(s t)^{2}\langle\overrightarrow{A B}, \overrightarrow{A B}\rangle_{A}}{|s t|^{2}\|\overrightarrow{A B}\|_{A}^{2}}=1
$$

But then Proposition 1.5 implies that $D={ }_{P} F$ as desired.

Recall that vector space $V$ has a unique vector $\mathbf{0}$ which is the identity element with respect to addition, i.e. $v+\mathbf{0}=v=\mathbf{0}+v=v$ for all $v \in V$. If we think of $\overrightarrow{A A}$ as an analog to the zero vector, then the following theorem implies that $\overrightarrow{A A}$ is a left additive identity for arrows that have the point $A$ as a tail.

Theorem 1.6. Given an arrow $\overrightarrow{A B}$ of $\mathscr{P}_{A}$, there is a a unique arrow $\overrightarrow{A A}$ (similarly, a unique arrow $\overrightarrow{B B}$ ) such that

$$
\overrightarrow{A A}+{ }_{A} \overrightarrow{A B}={ }_{A} \overrightarrow{A B}
$$

(and similarly $\overrightarrow{A B}+{ }_{A} \overrightarrow{B B}={ }_{A} \overrightarrow{A B}$ ). 
Proof : Let $\overrightarrow{A B}$ be an arrow in $\mathscr{P}_{A}$. Since $\overrightarrow{A A}$ is well defined by Definition 2, Definition 5 implies that $\overrightarrow{A A}+{ }_{A} \overrightarrow{A B}={ }_{A} \overrightarrow{A B}$. Now if there exists another arrow $\overrightarrow{C D}$ such that

$$
\overrightarrow{C D}+{ }_{A} \overrightarrow{A B}={ }_{A} \overrightarrow{A B}
$$

then since $\overrightarrow{A B}$ is fixed, Definition 5 implies that $D={ }_{P} A$. Thus, Equation (1.33) becomes

$$
\overrightarrow{C A}+{ }_{A} \overrightarrow{A B}={ }_{A} \overrightarrow{C B}
$$

Since Equations (1.33) and (1.34) are equivalent, comparing them yields $\overrightarrow{C B}={ }_{A} \overrightarrow{A B}$, which means (by Definition 3) that we must have $C={ }_{P} A$. Therefore, $\overrightarrow{C D}={ }_{A} \overrightarrow{A A}$.

For any $v \in V$, there is a unique element $-v$ (the additive inverse of $v$ ) such that $v+(-v)=-v+v=0$. The following theorem is the arrow space analog of the additive inverse.

Theorem 1.7. Let $\overrightarrow{A A}$ be fixed in $\mathscr{P}_{A}$. For any arrow $\overrightarrow{A B}$ in $\mathscr{P}_{A}$ there exists a unique arrow $\overrightarrow{B A}$ such that

$$
\overrightarrow{A B}+{ }_{A} \overrightarrow{B A}={ }_{A} \overrightarrow{A A}
$$

Proof : Let $\overrightarrow{A A}$ be fixed. For any point $B \in \mathscr{P}, \overrightarrow{A B}$ is in $\mathscr{P}_{A}$. By Definition $2, \overrightarrow{B A}$ is also in $\mathscr{P}_{A}$, and Definition 5 implies that

$$
\overrightarrow{A B}+{ }_{A} \overrightarrow{B A}={ }_{A} \overrightarrow{A A}
$$

Now, if exists another arrow $\overrightarrow{C D}$ such that

$$
\overrightarrow{A B}+{ }_{A} \overrightarrow{C D}={ }_{A} \overrightarrow{A A}
$$

then since $\overrightarrow{A B}$ is fixed, the only way for $\overrightarrow{A B}+{ }_{A} \overrightarrow{C D}$ to be defined is to have $C={ }_{P} B$. Thus, if we change $C$ into $B$ in the Equation (1.36) and use Definition 5, we would have

$$
\overrightarrow{A B}+{ }_{A} \overrightarrow{B D}={ }_{A} \overrightarrow{A D}
$$

Since Equations (1.36) and (1.37) are equivalent, comparing them yields $\overrightarrow{A D}={ }_{A} \overrightarrow{A A}$ which means ( by Definition 3), that $D={ }_{P} A$. Thus, $\overrightarrow{C D}={ }_{A} \overrightarrow{B A}$.

The following proposition, the arrows space analog of the vector space property $s 0=0$, is a direct consequence of Definition 7 .

Proposition 1.6. For any arrow $\overrightarrow{A A}$ in $\mathscr{P}_{A}$ and any $s \in \mathbb{R},(s) \overrightarrow{A A}={ }_{A} \overrightarrow{A A}$. 
In a vector space $V$, given $v \in V$, there is a unique scalar, denoted 1 , such that $1 v=v$. The arrow scalar multiplication identity in an arrow space $\mathscr{P}_{A}$ can also be identified as follows.

Theorem 1.8. For any arrow $\overrightarrow{A B}$ in an arrow space $\mathscr{P}_{A},(1) \overrightarrow{A B}={ }_{A} \overrightarrow{A B}$.

Proof : If $A={ }_{P} B$, then the result follows from Proposition 1.6. So assume $A \neq{ }_{P} B$. Lemma 1.1 implies that $\|\overrightarrow{A B}\|_{A} \neq 0$. Definition 7 implies that $(1) \overrightarrow{A B}=\overrightarrow{A D}$ for some point $D$ such that

$$
\|\overrightarrow{A D}\|=\|(1) \overrightarrow{A B}\|_{A}=\|\overrightarrow{A B}\|_{A}
$$

and

$$
\left\langle\frac{\overrightarrow{A D}}{\|\overrightarrow{A D}\|}, \frac{\overrightarrow{A B}}{\|\overrightarrow{A B}\|_{A}}\right\rangle_{A}=1
$$

From Equations (1.38), and (1.39) we would like to conclude that $D={ }_{P} B$. But this will follow from the uniqueness of the point $D$ as referenced by the remark that followed Definition 7.

In a vector space $V$, multiplication by the scalar 0 always returns the zero vector, i.e. $0 v=\mathbf{0}$ for any $v \in V$. We end this section with the following proposition which provides the arrow space analog, where arrows of the form $\overrightarrow{A A}$ correspond to the zero vector.

Proposition 1.7. For any arrow $\overrightarrow{A B}$ in $\mathscr{P}_{A}$, $(0) \overrightarrow{A B}={ }_{A} \overrightarrow{A A}$.

Proof : Let $\overrightarrow{A B}$ be an arrow in $\mathscr{P}_{A}$. By Definition 7, (0) $\overrightarrow{A B}={ }_{A} \overrightarrow{A A}$.

\subsection{Lines in an Arrow Space}

The line is a crucial concept of Euclidean geometry since it appears within three of Euclid's original five axioms. Because an arrow space is to be the precursor of the Euclidean plane reinterpreted as a two-dimensional vector space, we will need to define what is meant by a line $l$ within an abstract arrow space $\mathscr{P}_{A}$. For any fixed $\overrightarrow{A B} \in \mathscr{P}_{A}$, we can use scalar multiplication to define a set of points with $\mathscr{P}_{A}$ determined by the "direction" of $\overrightarrow{A B}$. This aforementioned set of points in $\mathscr{P}_{A}$ is the line $l$. This set of points on $l$, namely $\mathscr{P}_{l}$, gives rise to a one-dimensional arrow space $\mathscr{P}_{l_{A}}$ contained within $\mathscr{P}_{A}$. When considered as an independent arrow space apart from $\mathscr{P}_{A}$, the arrow space $\mathscr{P}_{l_{A}}$ has the property that parallel postulate of the Euclidean plane is no longer an axiom but a theorem; see Subsection 1.4.3. This surprising result justifies an analysis of $\mathscr{P}_{l_{A}}$ in its own right and is focus of the two subsections contained within this section. But all of this analysis hinges on the definition and existence of a line within an arrow space. Thus, we begin with this pivotal definition. 
Definition 8. Let $\mathscr{P}_{A}$ be a given arrow space. Given a fixed point $A$ and an arrow $\overrightarrow{A B} \in \mathscr{P}_{A}$ with $A \neq_{P} B$, a line containing $A$ and $B$, denoted by $l_{A B}$ or $l$, is the set of all heads of arrows $\overrightarrow{A C}$ such that there exists $t \in \mathbb{R}$ with $\overrightarrow{A C}={ }_{A}(t) \overrightarrow{A B}$. The set of points on line $l$ is denoted by $\mathscr{P}_{l}$. The set of arrows determined by $\mathscr{P}_{l}$ is the arrow space $\mathscr{P}_{l_{A}}$.

To justify the existence of the line $l$ between any two distinct points $A$ and $B$ of $\mathscr{P}$, we consider the next axiom which matches points on a line with the real numbers.

Axiom 3. Let $l$ be a line containing the points $A$ and $B$. There exists a one-to-one correspondence between the set of real numbers and the set of points that lie on $l$.

Axiom 3 shows that the line $l_{A B}$ is a nonempty set of $\mathscr{P}$; see the proof of Theorem 1.11. Furthermore, since the real line is a totally ordered set, Axiom 3 provides a geometric intuition of order (or betweenness) of points in $\mathscr{P}_{l}$ associated with the line $l$. The official algebraic definition of this geometric intuition is provided below.

Definition 9. Let $A, B$, and $C$ be any three distinct points on a line $l$. We say that the point $B$ is between $A$ and $C$ if $\left\langle\frac{\overrightarrow{B A}}{\|\overrightarrow{B A}\|_{A}}, \frac{\overrightarrow{B C}}{\|\overrightarrow{B C}\|_{A}}\right\rangle_{A}=-1$. If $B$ is between $A$ and $C$, we write $A \prec B \prec C$.

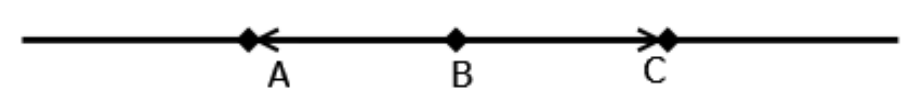

Figure 1.4: A depiction of $A \prec B \prec C$, where $A, B$, and $C$ are three points on the line $l$.

The notion of betweenness provided by Definition 9, along with the correspondence between the real line and the line $l$ determined by $\overrightarrow{A B}$, allows us to illustrate an abstract arrow $\overrightarrow{A B}$ as a directed finite line segment which starts at the tail $A$ and ends at head $B$, where the direction is recorded by the placement of an arrowhead on the head point $B$. We have been using this illustration convention throughout the previous sections even though we had no concept of betweenness and an arrow $\overrightarrow{A B}$, in all rigor, should have been depicted as two discrete points $A$ and $B$.

\subsubsection{Existence of Line}

We are interested in proving an arrow space analog of Euclid's first axiom, namely that given any two distinct points, there exists a unique line which contains the two given points; see Theorem 1.11. Before we do this, we introduce a proposition which 
is used in the proof of Theorem 1.11. This proposition provides a pre-inner product constraint for two unit arrows which lie on the same line, namely that the unit arrows have the same direction (pre-inner product is 1) or that the unit arrows have opposite directions (pre-inner product is -1 ).

Lemma 1.3. If $A, B, M$, and $L$ are four distinct points of line $l_{A B}$, then

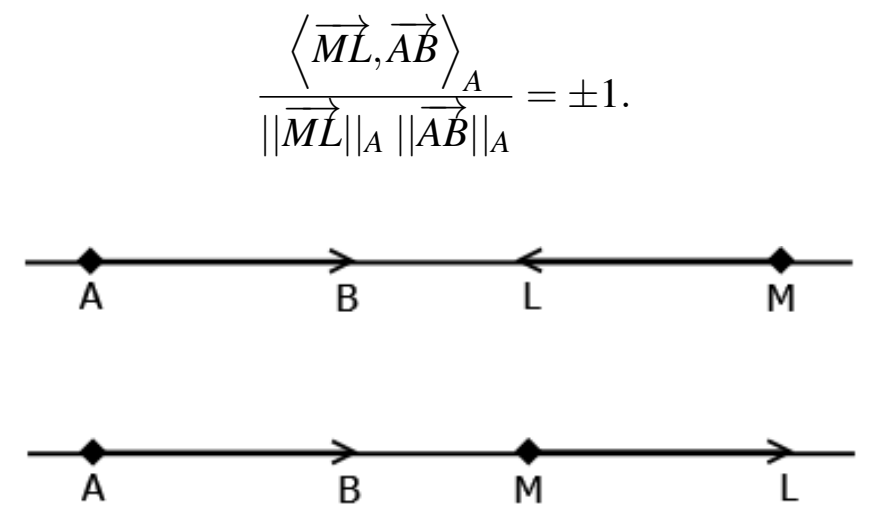

Figure 1.5: Points on the line $l_{A B}$ illustrating Equation (1.40).

Proof : Let $A, B, M$, and $L$ be four distinct points of line $l_{A B}$. By Definition 8 there exists $t_{M}, t_{L} \in \mathbb{R}$ such that

$$
\overrightarrow{A M}={ }_{A}\left(t_{M}\right) \overrightarrow{A B}, \quad \overrightarrow{A L}={ }_{A}\left(t_{L}\right) \overrightarrow{A B}
$$

which when combined with Definition 4 implies that

$$
\overrightarrow{M A}={ }_{A}-\left(\left(t_{M}\right) \overrightarrow{A B}\right)
$$

Since $\overrightarrow{M A}+{ }_{A} \overrightarrow{A L}={ }_{A} \overrightarrow{M L}$, we find through various applications of Axioms 1 and 2 that

$$
\frac{\langle\overrightarrow{M L}, \overrightarrow{A B}\rangle_{A}}{\|\overrightarrow{M L}\|_{A}\|\overrightarrow{A B}\|_{A}}=\frac{\left(-t_{M}+t_{L}\right)\langle\overrightarrow{A B}, \overrightarrow{A B}\rangle_{A}}{\left\|\overrightarrow{M A}+{ }_{A} \overrightarrow{A L}\right\|_{A}\|\overrightarrow{A B}\|_{A}}
$$

We can also simplify the denominator of Equation (1.43) in a similar manner, namely

$$
\begin{aligned}
\left\|\overrightarrow{M A}+{ }_{A} \overrightarrow{A L}\right\|_{A} & =\sqrt{\left\langle\overrightarrow{M A}+{ }_{A} \overrightarrow{A L}, \overrightarrow{M A}+{ }_{A} \overrightarrow{A L}\right\rangle_{A}} \\
& =\sqrt{\left(t_{M}^{2}-2 t_{M} t_{L}+t_{L}^{2}\right)\langle\overrightarrow{A B}, \overrightarrow{A B}\rangle_{A}}=\sqrt{\left(t_{L}-t_{M}\right)^{2}}\|\overrightarrow{A B}\|_{A}
\end{aligned}
$$


Equation (1.44) implies that

$$
\left.\left\|\overrightarrow{M A}+{ }_{A} \overrightarrow{A L}||_{A}\right\| \overrightarrow{A B}\left\|_{A}=\left|t_{L}-t_{M}\right|\right\| \overrightarrow{A B}\right|_{A} ^{2}
$$

Plugging the result of Equation (1.45) (notice that $t_{L}-t_{M} \neq 0$ since otherwise we would have $L={ }_{P} M$ which is a contradiction to the hypotheses of this lemma) into the right hand side of the Equation (1.43) yields

$$
\frac{\langle\overrightarrow{M L}, \overrightarrow{A B}\rangle_{A}}{\|\overrightarrow{M L}\|_{A}\|\overrightarrow{A B}\|_{A}}=\frac{\left(t_{L}-t_{M}\right)\|\overrightarrow{A B}\|_{A}^{2}}{\left|t_{L}-t_{M}\right|\|\overrightarrow{A B}\|_{A}^{2}}= \pm 1
$$

We next provide a pre-inner product condition for determining when a given point lies on a fixed line; see Theorems 1.9 and 1.10. Since the set of points $\mathscr{P}_{l}$ of $l_{A B}$ is contained within underlying the set of points $\mathscr{P}$ of $\mathscr{P}_{A}$, we can examine the following situation. Suppose for some point $D \in \mathscr{P}$ distinct from $A$ and $B$, we have $\frac{\langle\overrightarrow{A B}, \overrightarrow{A D}\rangle_{A}}{\|\overrightarrow{A B}\|_{A}\|\overrightarrow{A D}\|_{A}}= \pm 1$, then $D \in \mathscr{P}_{l}$, see Figures 1.6 and 1.9.

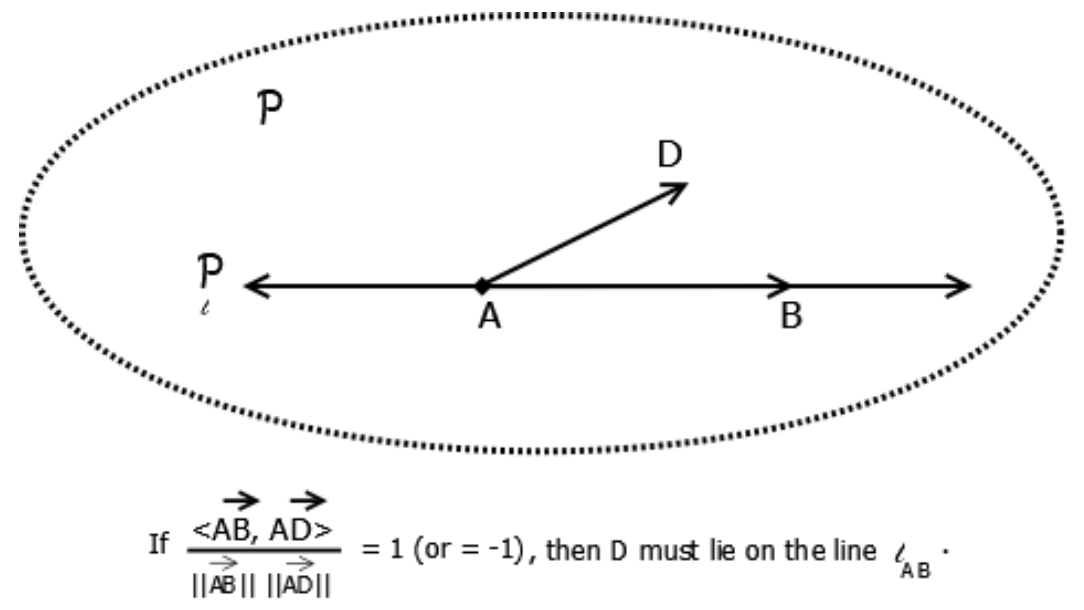

Figure 1.6: An illustration for when a given point lies on a fixed line.

Theorem 1.9. Let $A, B, D$ be three distinct points of $\mathscr{P}$ such that $\frac{\langle\overrightarrow{A B}, \overrightarrow{A D}\rangle_{A}}{\|\overrightarrow{A B}\|_{A}\|\overrightarrow{A D}\|_{A}}=1$. Then

1.

$$
\frac{\langle\overrightarrow{A B}, \overrightarrow{B D}\rangle}{\|\overrightarrow{A B}\|_{A}\|\overrightarrow{B D}\|_{A}}= \pm 1
$$


2. either

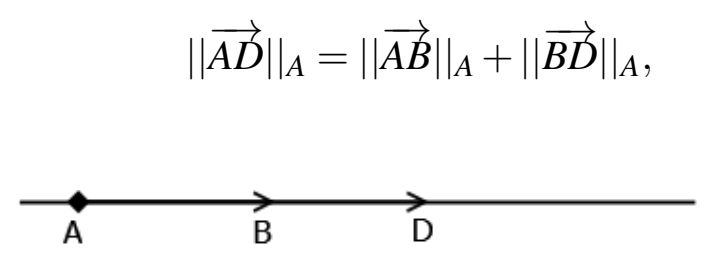

Figure 1.7: A depiction of three points $A, B, D$ where $\|\overrightarrow{A D}\|_{A}=\|\overrightarrow{A B}\|_{A}+\|\overrightarrow{B D}\|_{A}$.

or

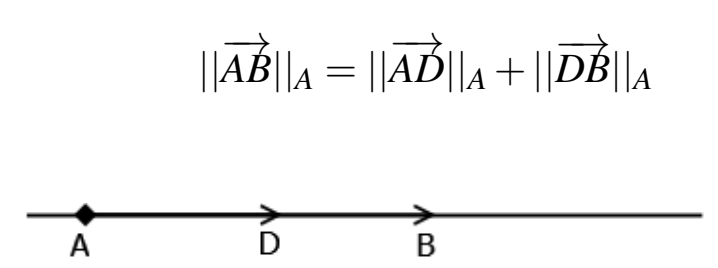

Figure 1.8: A depiction of three points $A, B, D$ where $\|\overrightarrow{A B}\|_{A}=\|\overrightarrow{A D}\|_{A}+\|\overrightarrow{D B}\|_{A}$.

3. there exists $t_{D} \in \mathbb{R}$ with $t_{D}>0$ such that $\overrightarrow{A D}={ }_{A}\left(t_{D}\right) \overrightarrow{A B}$; thus the point $D$ lies on the line $l_{A B}$; see Figure 5.4.

\section{Proof :}

1. First notice that it is not given that $A, B$, and $D$ lie on the same line; in fact, we need to prove this. So let $A, B$, and $D$ be three distinct points of $\mathscr{P}$ such that

$$
\frac{\langle\overrightarrow{A B}, \overrightarrow{A D}\rangle_{A}}{\|\overrightarrow{A B}\|_{A}\|\overrightarrow{A D}\|_{A}}=1
$$

Since $\overrightarrow{A D}={ }_{A} \overrightarrow{A B}+{ }_{A} \overrightarrow{B D}$, applications of Axiom 1 and Definition 6 to Equation (1.46) implies that

$$
1=\frac{\left\langle\overrightarrow{A B}, \overrightarrow{A B}+{ }_{A} \overrightarrow{B D}\right\rangle_{A}}{\|\overrightarrow{A B}\|_{A}\|\overrightarrow{A D}\|_{A}}=\frac{\|\overrightarrow{A B}\|_{A}^{2}+\langle\overrightarrow{A B}, \overrightarrow{B D}\rangle_{A}}{\|\overrightarrow{A B}\|_{A}\|\overrightarrow{A D}\|_{A}}
$$

Multiplying both sides in the above by $\|\overrightarrow{A B}\|_{A}\|\overrightarrow{A D}\|_{A}$ yields

$$
\|\overrightarrow{A B}\|_{A}^{2}+\langle\overrightarrow{A B}, \overrightarrow{B D}\rangle_{A}=\|\overrightarrow{A B}\|_{A}\|\overrightarrow{A D}\|_{A}
$$


Similar calculations show that

$$
\|\overrightarrow{A D}\|_{A}=\sqrt{\left\langle\overrightarrow{A B}+{ }_{A} \overrightarrow{B D}, \overrightarrow{A B}+{ }_{A} \overrightarrow{B D}\right\rangle_{A}}=\sqrt{\|\overrightarrow{A B}\|_{A}^{2}+2\langle\overrightarrow{A B}, \overrightarrow{B D}\rangle_{A}+\|\overrightarrow{B D}\|_{A}^{2}}
$$

If we combine Equations (1.47) and (1.48), we get

$$
\|\overrightarrow{A B}\|_{A}^{2}+\langle\overrightarrow{A B}, \overrightarrow{B D}\rangle_{A}=\|\overrightarrow{A B}\|_{A} \sqrt{\|\overrightarrow{A B}\|_{A}^{2}+2\langle\overrightarrow{A B}, \overrightarrow{B D}\rangle_{A}+\|\overrightarrow{B D}\|_{A}^{2}}
$$

By squaring both sides of the above, we get

$$
\begin{aligned}
\|\overrightarrow{A B}\|_{A}^{4} & +2\|\overrightarrow{A B}\|_{A}^{2}\langle\overrightarrow{A B}, \overrightarrow{B D}\rangle_{A}+\langle\overrightarrow{A B}, \overrightarrow{B D}\rangle^{2} \\
& =\|\overrightarrow{A B}\|_{A}^{4}+2\|\overrightarrow{A B}\|_{A}^{2}\langle\overrightarrow{A B}, \overrightarrow{B D}\rangle_{A}+\|\overrightarrow{A B}\|_{A}^{2}\|\overrightarrow{B D}\|_{A}^{2},
\end{aligned}
$$

which is equivalent to

$$
\langle\overrightarrow{A B}, \overrightarrow{B D}\rangle^{2}=\|\overrightarrow{A B}\|_{A}^{2}\|\overrightarrow{B D}\|_{A}^{2}
$$

Taking the square root of both sides of the above gives

$$
\langle\overrightarrow{A B}, \overrightarrow{B D}\rangle= \pm\|\overrightarrow{A B}\|_{A}\|\overrightarrow{B D}\|_{A}
$$

Since $A \neq_{P} B, B \neq_{P} D$, it follows by Lemma 1.1 that $\|\overrightarrow{A B}\|_{A} \neq 0,\|\overrightarrow{B D}\|_{A} \neq 0$. Hence, if we divide both sides of the Equation (1.50) by $\|\overrightarrow{A B}\|_{A}\|\overrightarrow{B D}\|_{A}$, we get the desired equation, that is

$$
\frac{\langle\overrightarrow{A B}, \overrightarrow{B D}\rangle}{\|\overrightarrow{A B}\|_{A}\|\overrightarrow{B D}\|_{A}}= \pm 1
$$

2. To prove the second part of the theorem we return to Equation (1.46) and work on expanding the numerator $\langle\overrightarrow{A B}, \overrightarrow{A D}\rangle_{A}$ via Axiom 1 and the fact that $\overrightarrow{A D}={ }_{A}$ $\overrightarrow{A B}+{ }_{A} \overrightarrow{B D}$ and $\overrightarrow{A B}={ }_{A} \overrightarrow{A D}+{ }_{A} \overrightarrow{D B}$. Equation (1.46) may then be rewritten as

$$
\begin{aligned}
\|\overrightarrow{A B}\|_{A}\|\overrightarrow{A D}\|_{A} & =\langle\overrightarrow{A B}, \overrightarrow{A D}\rangle_{A}=\left\langle\overrightarrow{A D}+_{A} \overrightarrow{D B}, \overrightarrow{A B}+{ }_{A} \overrightarrow{B D}\right\rangle_{A} \\
& =\langle\overrightarrow{A D}, \overrightarrow{A B}\rangle_{A}+\langle\overrightarrow{A D}, \overrightarrow{B D}\rangle_{A}+\langle\overrightarrow{D B}, \overrightarrow{A B}\rangle_{A}+\langle\overrightarrow{D B}, \overrightarrow{B D}\rangle_{A} \\
& =\langle\overrightarrow{A D}, \overrightarrow{A D}\rangle_{A}+\langle\overrightarrow{D B}, \overrightarrow{A B}\rangle_{A}+\langle\overrightarrow{D B}, \overrightarrow{B D}\rangle_{A} \\
& =\langle\overrightarrow{A D}, \overrightarrow{A D}\rangle_{A}+\langle\overrightarrow{D B}, \overrightarrow{A B}\rangle_{A}-\langle\overrightarrow{B D}, \overrightarrow{B D}\rangle_{A} \\
& =\|\overrightarrow{A D}\|_{A}^{2}+\langle\overrightarrow{D B}, \overrightarrow{A B}\rangle_{A}-\|\overrightarrow{B D}\|_{A}^{2}
\end{aligned}
$$


The next step is to manipulate Equation (1.50) through Definition 4 and repeated applications of Axiom 1. Definition 4 implies that $\overrightarrow{B D}={ }_{A}-\overrightarrow{D B}$. Thus, it follows by Equation (1.6) and Equation (1.2) that

$$
\langle\overrightarrow{A B}, \overrightarrow{B D}\rangle=\langle\overrightarrow{B D}, \overrightarrow{A B}\rangle=-\langle\overrightarrow{A B}, \overrightarrow{D B}\rangle
$$

This means that we can rewrite Equation (1.50) (noticing that $\|\overrightarrow{D B}\|_{A}=\|\overrightarrow{B D}\|_{A}$ by Equation (1.10)) as follows

$$
\langle\overrightarrow{D B}, \overrightarrow{A B}\rangle=\mp\|\overrightarrow{A B}\|_{A}\|\overrightarrow{D B}\|_{A}
$$

If we combine Equations (1.52) and (1.53), we get

$$
\|\overrightarrow{A D}\|_{A}^{2} \mp\|\overrightarrow{A B}\|_{A}\|\overrightarrow{D B}\|_{A}-\|\overrightarrow{D B}\|_{A}^{2}=\|\overrightarrow{A B}\|_{A}\|\overrightarrow{A D}\|_{A}
$$

Rearranging the above equation yields

$$
\|\overrightarrow{A D}\|_{A}^{2}-\|\overrightarrow{D B}\|_{A}^{2}=\|\overrightarrow{A B}\|_{A}\|\overrightarrow{A D}\|_{A} \pm\|\overrightarrow{A B}\|_{A}\|\overrightarrow{D B}\|_{A}
$$

that is,

$$
\left(\|\overrightarrow{A D}\|_{A}-\|\overrightarrow{D B}\|_{A}\right)\left(\|\overrightarrow{A D}\|_{A}+\|\overrightarrow{D B}\|_{A}\right)=\|\overrightarrow{A B}\|_{A}\left(\|\overrightarrow{A D}\|_{A} \pm\|\overrightarrow{D B}\|_{A}\right)
$$

Now, Equation (1.54) tells us that either

$$
\begin{aligned}
& \|\overrightarrow{A D}\|_{A}-\|\overrightarrow{B D}\|_{A}=\|\overrightarrow{A B}\|_{A}, \quad \text { or } \\
& \|\overrightarrow{A D}\|_{A}+\|\overrightarrow{D B}\|_{A}=\|\overrightarrow{A B}\|_{A},
\end{aligned}
$$

as desired.

3. Notice that, when proving Part 2 either Equation (1.55) or Equation (1.56), is valid. We will show one case, the case of Equation (1.55) being valid, and leave the case of Equation (1.56) to the reader. Since $A \neq_{P} B$, Lemma 1.1 implies that $\|\overrightarrow{A B}\|_{A} \neq 0$. Hence, Equation (1.55) becomes $\|\overrightarrow{A D}\|_{A}=\|\overrightarrow{A B}\|_{A}\left(1+\frac{\|\overrightarrow{B D}\|_{A}}{\|\left.\overrightarrow{A B}\right|_{A}}\right)$. If we put

$$
t_{D}=1+\frac{\|\overrightarrow{B D}\|_{A}}{\|\overrightarrow{A B}\|_{A}},
$$

then we can rewrite the preceding equation as $\|\overrightarrow{A D}\|_{A}=\left(t_{D}\right)\|\overrightarrow{A B}\|_{A}$. Notice that, $t_{D}>0$ as $\|\overrightarrow{B D}\|_{A},\|\overrightarrow{A B}\|_{A}>0$. Hence, by Equation (1.9) we have

$$
\|\overrightarrow{A D}\|_{A}=\left(t_{D}\right)\|\overrightarrow{A B}\|_{A}=\left\|\left(t_{D}\right) \overrightarrow{A B}\right\|_{A}
$$


Also, by Equations (1.6) and (1.9) we have

$$
\frac{\left\langle\overrightarrow{A D},\left(t_{D}\right) \overrightarrow{A B}\right\rangle}{\|\overrightarrow{A D}\|_{A}\left\|\left(t_{D}\right) \overrightarrow{A B}\right\|_{A}}=\frac{t_{D}\langle\overrightarrow{A D}, \overrightarrow{A B}\rangle}{t_{D}\|\overrightarrow{A D}\|_{A}\|\overrightarrow{A B}\|_{A}}=\frac{\langle\overrightarrow{A D}, \overrightarrow{A B}\rangle}{\|\overrightarrow{A D}\|_{A}\|\overrightarrow{A B}\|_{A}}=1
$$

where the last equality follows by the hypothesis of this theorem. By Definition 7 we have

$$
\left(t_{D}\right) \overrightarrow{A B}={ }_{A} \overrightarrow{A K}
$$

for some point $K$, and by Definition 8 the point $K$ lies on the line $l_{A B}$. Equation (1.57) implies that $\|\overrightarrow{A D}\|=\|\overrightarrow{A K}\|$ while Equation (1.58) implies that $\frac{\langle\overrightarrow{A D}, \overrightarrow{A K}\rangle}{\|\overrightarrow{A D}\|_{A}\|\overrightarrow{A K}\|_{A}}=1$. Thus, Proposition 1.5 is applicable and shows that $D={ }_{P} K$, a point on $l_{A B}$.

An analogous result occurs when the postulated inner product in the hypothesis of Theorem 1.9 is set equal to -1 .

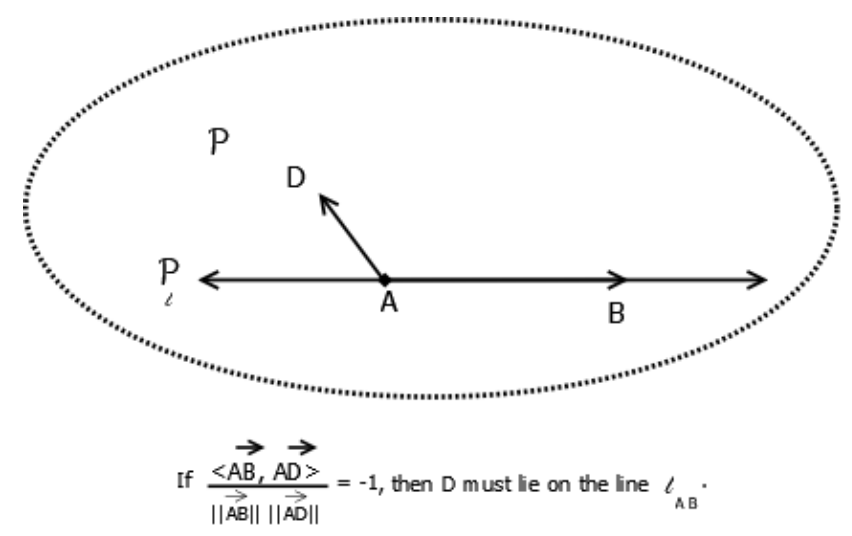

Figure 1.9: An illustration of Theorem 1.10.

Theorem 1.10. Let $A, B$, and $D$ be three distinct points of $\mathscr{P}$ such that $\frac{\langle\overrightarrow{A B}, \overrightarrow{A D}\rangle_{A}}{\|\overrightarrow{A B}\|_{A}\|\overrightarrow{A D}\|_{A}}=$ -1 . Then

1.

$$
\frac{\langle\overrightarrow{A B}, \overrightarrow{B D}\rangle}{\|\overrightarrow{A B}\|_{A}\|\overrightarrow{B D}\|_{A}}= \pm 1
$$

2.

$$
\|\overrightarrow{A D}\|_{A}=-\|\overrightarrow{A B}\|_{A}+\|\overrightarrow{B D}\|_{A}
$$




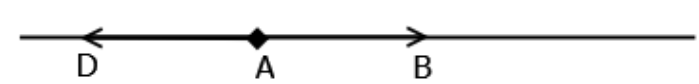

Figure 1.10: A depiction of three points $A, B, D$ where $\|\overrightarrow{A D}\|_{A}=-\|\overrightarrow{A B}\|_{A}+\|\overrightarrow{B D}\|_{A}$.

3. there exists $t_{D} \in \mathbb{R}$ with $t_{D}<0$ such that $\overrightarrow{A D}={ }_{A}\left(t_{D}\right) \overrightarrow{A B}$; thus the point $D$ lies on the line $l_{A B}$; see Figure 1.9.

Proof : The proof of Part 1 is the same as the proof of Part 1 of Theorem 1.9 and is left to the reader. The proof of Part 2 is slightly more subtle. Since $\langle\overrightarrow{A B}, \overrightarrow{A D}\rangle_{A}=$ $-\|\overrightarrow{A B}\|_{A}\|\overrightarrow{A D}\|_{A}$, Equation (1.54) becomes

$$
\left(\|\overrightarrow{A D}\|_{A}-\|\overrightarrow{D B}\|_{A}\right)\left(\|\overrightarrow{A D}\|_{A}+\|\overrightarrow{D B}\|_{A}\right)=-\|\overrightarrow{A B}\|_{A}\left(\|\overrightarrow{A D}\|\left\|_{A} \mp\right\| \overrightarrow{D B} \|_{A}\right)
$$

A priori, it seems like Equation (1.60) has two possibilities. However, if we choose the minus sign option, Equation (1.60) becomes

$$
\left(\|\overrightarrow{A D}\|_{A}-\|\overrightarrow{D B}\|_{A}\right)\left(\|\overrightarrow{A D}\|_{A}+\|\overrightarrow{D B}\|_{A}\right)=-\|\overrightarrow{A B}\|_{A}\left(\|\overrightarrow{A D}\|_{A}-\|\overrightarrow{D B}\|_{A}\right)
$$

which leads to the contradiction of $-\|\overrightarrow{A B}\|=\|\overrightarrow{A D}\|_{A}+\|\overrightarrow{D B}\|_{A}$. Thus, Equation (1.60) really is

$$
\left(\|\overrightarrow{A D}\|_{A}-\|\overrightarrow{D B}\|_{A}\right)\left(\|\overrightarrow{A D}\|_{A}+\|\overrightarrow{D B}\|_{A}\right)=-\|\overrightarrow{A B}\|_{A}\left(\|\overrightarrow{A D}\|_{A}+\|\overrightarrow{D B}\|_{A}\right)
$$

which is equivalent to

$$
\|\overrightarrow{A D}\|_{A}-\|\overrightarrow{B D}\|_{A}=-\|\overrightarrow{A B}\|_{A}
$$

For the proof of Part 3 we need to first rewrite Equation (1.61) as

$$
\|\overrightarrow{A D}\|_{A}+\|\overrightarrow{A B}\|_{A}=\|\overrightarrow{B D}\|_{A}
$$

Equation (1.62) shows that $\|\overrightarrow{A B}\|_{A} \leq\|\overrightarrow{B D}\|_{A}$, which in turn implies

$$
1 \leq \frac{\|\overrightarrow{B D}\|_{A}}{\|\overrightarrow{A B}\|_{A}}
$$

Also, Equation (1.61) implies that $-\|\overrightarrow{A D}\|_{A}=\|\overrightarrow{A B}\|_{A}\left(1-\frac{\|\overrightarrow{B D}\|_{A}}{\|\overrightarrow{A B}\|_{A}}\right)$. If we put

$$
t_{D}=1-\frac{\|\overrightarrow{B D}\|_{A}}{\|\overrightarrow{A B}\|_{A}}
$$


then we can rewrite the preceding equation as $-\|\overrightarrow{A D}\|_{A}=\left(t_{D}\right)\|\overrightarrow{A B}\|_{A}$. Equation (1.63) implies $t_{D} \leq 0$. However if $t_{D}=0$, we get a contradiction since by hypothesis $A$ is distinct from $D$. So in fact $t_{D}<0$. Then

$$
\frac{\left\langle\overrightarrow{A D},\left(t_{D}\right) \overrightarrow{A B}\right\rangle}{\|\overrightarrow{A D}\|_{A}\left\|\left(t_{D}\right) \overrightarrow{A B}\right\|_{A}}=\frac{t_{D}\langle\overrightarrow{A D}, \overrightarrow{A B}\rangle}{\left|t_{D}\right|\|\overrightarrow{A D}\|_{A}\|\overrightarrow{A B}\|_{A}}=-\frac{\langle\overrightarrow{A D}, \overrightarrow{A B}\rangle}{\|\overrightarrow{A D}\|_{A}\|\overrightarrow{A B}\|_{A}}
$$

Since by the hypothesis $\frac{\langle\overrightarrow{A D}, \overrightarrow{A B}\rangle}{\|\overrightarrow{A D}\|_{A}|| \overrightarrow{A B} \|_{A}}=-1$, the equation in the above simplifies to

$$
\frac{\left\langle\overrightarrow{A D},\left(t_{D}\right) \overrightarrow{A B}\right\rangle}{\|\overrightarrow{A D}\|_{A}\left\|\left(t_{D}\right) \overrightarrow{A B}\right\|_{A}}=1
$$

Furthermore

$$
\|\overrightarrow{A D}\|_{A}=-\left(t_{D}\right)\|\overrightarrow{A B}\|_{A}=\left|t_{D}\right|\|\overrightarrow{A B}\|_{A}=\left\|\left(t_{D}\right) \overrightarrow{A B}\right\|_{A}
$$

and the rest of the argument proceeds analogously to proof of Part 3 of Theorem 1.9.

Finally we are in the position to prove the arrow space equivalent of Euclid's first axiom, namely that for any two distinct points there exists a unique line that contains the two points.

Theorem 1.11. (The Axiom II of Incidence) Given any two distinct points $A$ and $B$ of $\mathscr{P}$, there exists a unique line $l_{A B}$ containing $A$ and $B$.

Proof : Let $A$ and $B$ be two distinct points of $\mathscr{P}$. It follows by Axiom 3 that the set $\left\{F \mid \overrightarrow{A F}={ }_{A}(t) \overrightarrow{A B}\right.$, where $\left.t \in \mathbb{R}\right\}$ is non-empty. By Definition 8 this set forms a line containing $A$ and $B$. To show the uniqueness part of this theorem, let $l_{A B}=$ $\left\{F \mid \overrightarrow{A F}={ }_{A}(t) \overrightarrow{A B}\right.$, where $\left.t \in \mathbb{R}\right\}$ and $l_{M L}=\left\{K \mid \overrightarrow{M K}={ }_{A}(s) \overrightarrow{M L}\right.$, where $\left.s \in \mathbb{R}\right\}$ be two lines each of which contains $A$ and $B$, where $M$ and $L$ are any two distinct points that lie on $l_{A B}$ other than $A$ and $B$. By Definition 8 there exists $t_{M}, t_{L} \in \mathbb{R}$ such that

$$
\overrightarrow{A M}={ }_{A}\left(t_{M}\right) \overrightarrow{A B}, \overrightarrow{A L}=_{A}\left(t_{L}\right) \overrightarrow{A B}
$$

To show that $l_{A B}=l_{M L}$, let $D \in l_{M L}$. It follows by Definition 8 that there exists $s_{D} \in \mathbb{R}$ with

$$
\overrightarrow{M D}={ }_{A}\left(s_{D}\right) \overrightarrow{M L}
$$

See Figure 1.11.

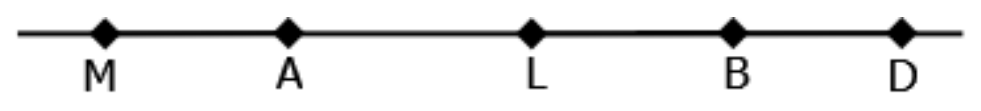

Figure 1.11: The relationship between points $M, A, L, B$, and $D$ on a line $l_{A B}$. 
In order to show that $D \in l_{A B}$, we need to find some $t_{D} \in \mathbb{R}$ such that $\overrightarrow{A D}={ }_{A}$ $\left(t_{D}\right) \overrightarrow{A B}$. Since $A, B, M$, and $L$ satisfy the hypotheses of Lemma 1.3 , we have

$$
\frac{\langle\overrightarrow{M L}, \overrightarrow{A B}\rangle_{A}}{\|\overrightarrow{M L}\|_{A}\|\overrightarrow{A B}\|_{A}}= \pm 1
$$

which is equivalent to

$$
\langle\overrightarrow{M L}, \overrightarrow{A B}\rangle_{A}= \pm\|\overrightarrow{M L}\|_{A}\|\overrightarrow{A B}\|_{A}
$$

Now, since $\overrightarrow{A D}={ }_{A} \overrightarrow{A M}+{ }_{A} \overrightarrow{M D}$, it follows by Equation (1.5) and Definition 6 that

$$
\frac{\langle\overrightarrow{A D}, \overrightarrow{A B}\rangle_{A}}{\|\overrightarrow{A D}\|_{A}\|\overrightarrow{A B}\|_{A}}=\frac{\langle\overrightarrow{A M}, \overrightarrow{A B}\rangle_{A}+\langle\overrightarrow{M D}, \overrightarrow{A B}\rangle_{A}}{\sqrt{\langle\overrightarrow{A D}, \overrightarrow{A D}\rangle_{A}}\|\overrightarrow{A B}\|_{A}}
$$

The numerator of the right hand side in the Equation (1.69) can be rewritten, using Equations (1.66), (1.67), (1.68), Definition (6), and Equation (1.8) as follows

$$
\begin{aligned}
\langle\overrightarrow{A M}, \overrightarrow{A B}\rangle_{A}+\langle\overrightarrow{M D}, \overrightarrow{A B}\rangle_{A} & =t_{M}\|\overrightarrow{A B}\|_{A}^{2}+s_{D}\langle\overrightarrow{M L}, \overrightarrow{A B}\rangle_{A} \\
& =t_{M}\|\overrightarrow{A B}\|_{A}^{2} \pm s_{D}\|\overrightarrow{M L}\|_{A}\|\overrightarrow{A B}\|_{A}
\end{aligned}
$$

Next, we simplify the denominator of the right hand side of the Equation (1.69) through a series of similar calculations. Equations (1.5) and (1.2) imply that

$$
\langle\overrightarrow{A D}, \overrightarrow{A D}\rangle=\langle\overrightarrow{A M}, \overrightarrow{A M}\rangle_{A}+2\langle\overrightarrow{M D}, \overrightarrow{A M}\rangle_{A}+\langle\overrightarrow{M D}, \overrightarrow{M D}\rangle_{A}
$$

Using Equations (1.66), (1.67), (1.68), Definition 6, and Equation (1.8), the preceding equation can be rearranged as follows

$$
\begin{aligned}
\langle\overrightarrow{A D}, \overrightarrow{A D}\rangle & =t_{M}^{2}\|\overrightarrow{A B}\|_{A}^{2}+2 t_{M} s_{D}\langle\overrightarrow{M L}, \overrightarrow{A B}\rangle_{A}+s_{D}^{2}\|\overrightarrow{M L}\|_{A}^{2} \\
& =t_{M}^{2}\|\overrightarrow{A B}\|_{A}^{2} \pm 2 t_{M} s_{D}\|\overrightarrow{M L}\|_{A}\|\overrightarrow{A B}\|_{A}+s_{D}^{2}\|\overrightarrow{M L}\|_{A}^{2} \\
& =\left(t_{M}\|\overrightarrow{A B}\|_{A} \pm s_{D}\|\overrightarrow{M L}\|_{A}\right)^{2} .
\end{aligned}
$$

This means that the denominator of the right hand side of the Equation (1.69) is

$$
\sqrt{\langle\overrightarrow{A D}, \overrightarrow{A D}\rangle_{A}}\|\overrightarrow{A B}\|_{A}=\left|t_{M}\|\overrightarrow{A B}\|_{A}^{2} \pm s_{D}\|\overrightarrow{M L}\|_{A}\|\overrightarrow{A B}\|_{A}\right|
$$


By Combining Equations (1.69), (1.70), and (1.72) we find that

$$
\frac{\langle\overrightarrow{A D}, \overrightarrow{A B}\rangle_{A}}{\|\overrightarrow{A D}\|_{A} \|\left.\overrightarrow{A B}\right|_{A}}=\frac{t_{M}\|\overrightarrow{A B}\|_{A}^{2} \pm s_{D}\|\overrightarrow{M L}\|_{A}\|\overrightarrow{A B}\|_{A}}{\left|t_{M}\|\overrightarrow{A B}\|_{A}^{2} \pm s_{D}\|\overrightarrow{M L}\|_{A}\|\overrightarrow{A B}\|_{A}\right|}= \pm 1
$$

Now, by means of Theorem 1.9 and 1.10 , we conclude that $D$ is a point on $l_{A B}$. This means that $l_{A B} \subseteq l_{M L}$, and a similar argument shows that $l_{M L} \subseteq l_{A B}$ to conclude that $l_{A B}=l_{M L}$, as desired.

After confirming the existence of a line, we conclude from Axiom 3 the following corollary which is an analog to the second axiom of incidence from Hilbert's axioms of plane geometry, see Appendix .3. However, the third axiom of incidence, see Axiom 5, will be postulated in arrow spaces.

Corollary 1.2. (The Axiom I2 of Incidence) There exist at least two points on a line.

Proof : Let $l$ be a line in an arrow space. Then, it follows by Axiom 3 that for every $t \in \mathbb{R}$ exists a point $P$ that uniquely corresponds to $t$.

Theorem 1.11 and Definition 8 affirm that a point $P$ on a line can be described as a head of an arrow $(t) \overrightarrow{A B}$ where $A$ and $B$ are any two arbitrary distinct points on $l$. We derive the following proposition which provides a description of the pre-inner product of any two unit arrows that emanate from the same point and lie on a line.

Proposition 1.8. Let $A, B$, and $C$ be three distinct points on a line l. Then, it is always true that

$$
\left\langle\frac{\overrightarrow{A B}}{\|\overrightarrow{A B}\|_{A}}, \frac{\overrightarrow{A C}}{\|\overrightarrow{A C}\|_{A}}\right\rangle_{A}= \pm 1
$$

Proof: By the uniqueness of line from Theorem 1.11 and by means of Definition 8 we can write $\overrightarrow{A B}={ }_{A}(t) \overrightarrow{A C}$ for some real number $t$. Then, since $A \neq_{P} B$, we have by Lemma 1.1 that $t \neq 0$. Now, since $\overrightarrow{A B}={ }_{A}(t) \overrightarrow{A C}$ we use Equation (1.8) to write

$$
\left\langle\frac{\overrightarrow{A B}}{\|\overrightarrow{A B}\|_{A}}, \frac{\overrightarrow{A C}}{\|\overrightarrow{A C}\|_{A}}\right\rangle_{A}=\frac{\langle\overrightarrow{A B}, \overrightarrow{A C}\rangle_{A}}{\|\overrightarrow{A B}\|_{A}\|\overrightarrow{A C}\|_{A}}=\frac{\langle(t) \overrightarrow{A C}, \overrightarrow{A C}\rangle_{A}}{\|(t) \overrightarrow{A C}\|_{A}\|\overrightarrow{A C}\|_{A}},
$$

which simplifies by Equations (1.8), (1.9), and Definition 6 to

$$
\left\langle\frac{\overrightarrow{A B}}{\|\overrightarrow{A B}\|_{A}}, \frac{\overrightarrow{A C}}{\|\overrightarrow{A C}\|_{A}}\right\rangle_{A}=\frac{(t)\langle\overrightarrow{A C}, \overrightarrow{A C}\rangle_{A}}{|(t)|\|\overrightarrow{A C}\|_{A}\|\overrightarrow{A C}\|_{A}}=\frac{(t)\|\overrightarrow{A C}\|_{A}^{2}}{|(t)|\|\overrightarrow{A C}\|_{A}^{2}}= \pm 1
$$

where the product is 1 if $t>0$, and is -1 if $t<0$. 


\subsubsection{Equivalence Relation on $\mathscr{P}_{l_{A}}$}

Now, that we have the definition of the unique $l$ determined by $\overrightarrow{A B}$, we can restrict the pre-inner product of $\mathscr{P}_{A}$ to $\mathscr{P}_{l_{A}}$ and use this restricted pre-inner product to define an equivalence relation on the arrows of $\mathscr{P}_{l_{A}}$ which geometrically captures when two arrows have equal length and direction. Because the pre-inner product on $\mathscr{P}_{l_{A}}$ is the restriction of the pre-inner product of $\mathscr{P}_{A}$ to those arrows of $\mathscr{P}_{l_{A}}$, we will also use $\langle-,-\rangle_{A}$ to denoted the pre-inner product of $\mathscr{P}_{l_{A}}$.

The aforementioned construction is an internal construction for a pre-inner product on $\mathscr{P}_{l_{A}}$ reminiscent of describing the restricted the inner product structure of direct summand in a vector space. We could also describe an external construction for developing pre-inner product on $\mathscr{P}_{l_{A}}$. Instead of using restricted pre-inner product from $\mathscr{P}_{A}$, we focus on $\mathscr{P}_{l_{A}}$ as a separate arrow space and apply the constructions of Section 1.1 with a new pre-inner product, which denote as $\langle-,-\rangle_{A_{l}}$. This new pre-inner product must obey Axioms 1 and 2 and thus gives rises to a new definition on $P_{l_{A}}$ of scalar multiplication, which is denoted as $(a)_{A_{l}} \overrightarrow{A B}$.

Either construction provides the arrow space $\mathscr{P}_{l_{A}}$ with a pre-inner product structure which is compatible with the propositions and theorems of this and the next subsection. It is a matter of taste as to which construction is preferred by the reader. For simplicity, we will write our results using the notation of the internal/restricted construction, but the reader should note that substitution of $\langle-,-\rangle_{A}$ with $\langle-,-\rangle_{A_{l}}$ and $(a) \overrightarrow{A B}$ with $(a)_{A_{l}} \overrightarrow{A B}$ will not change the validity of the results.

The algebraic definition of an equivalence relation on an arrow space $\mathscr{P}_{l_{A}}$ is provided below and will be essential to proving the existence of a unique line between any two points and the existence of a parallel arrow. See Theorems 1.11 and 1.15 respectively.

Definition 10. Let $\overrightarrow{A B}$ and $\overrightarrow{C D}$ be two arrows in $\mathscr{P}_{l_{A}}$. We say that $\overrightarrow{A B} \Re_{l} \overrightarrow{C D}$, if and only if, either $A={ }_{P} B$ and $C={ }_{P} D$, or

1. $\|\overrightarrow{A B}\|_{A}=\|\overrightarrow{C D}\|_{A}$, and

2. $\left\langle\frac{\overrightarrow{A B}}{\|\overrightarrow{A B}\|_{A}}, \frac{\overrightarrow{C D}}{\|\overrightarrow{C D}\|_{A}}\right\rangle_{A}=1$

The following proposition and corollary are immediate consequences of Definition 10. They will play a role ins the proof of Theorem 1.12 .

Proposition 1.9. If $\overrightarrow{A B} \Re_{l} \overrightarrow{C D}$ with $A={ }_{P} B$, then $C={ }_{P} D$. 
Corollary 1.3. If $\overrightarrow{A B} \Re_{l} \overrightarrow{C D}$ with $A \neq_{P} B$, then $C \neq_{P} D$.

Our next goal is to show that $\mathfrak{R}_{l}$ is an equivalence relation. To do so we need to introduce the following two theorems.

Theorem 1.12. Let $l_{O P}$ be a line and $\mathscr{P}_{l}$ be the set of points on this line. Let $\overrightarrow{A B}, \overrightarrow{C D}, \overrightarrow{E F}$, and $\overrightarrow{G H}$ be in $\mathscr{P}_{l_{A}}$ such that $\overrightarrow{A B} \Re_{l} \overrightarrow{C D}$ and $\overrightarrow{E F} \Re_{l} \overrightarrow{G H}$. Then

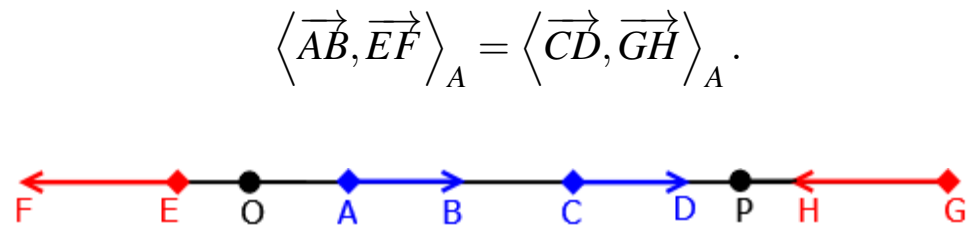

Figure 1.12: An illustration of Theorem 1.12. Note that the equivalence between arrows is color coded where equivalent arrows share the same color.

Proof : Let $\overrightarrow{A B}, \overrightarrow{C D}, \overrightarrow{E F}$, and $\overrightarrow{G H}$ be in $\mathscr{P}_{l_{A}}$ such that $\overrightarrow{A B} \Re_{l} \overrightarrow{C D}$ and $\overrightarrow{E F} \Re_{l} \overrightarrow{G H}$. The proof of this theorem will be divided into two parts. First, if $A={ }_{P} B$ (similarly if $C={ }_{P} D$ ), then since $\overrightarrow{A B} \mathfrak{R}_{l} \overrightarrow{C D}$, Proposition 1.9 implies that $C={ }_{P} D$. Hence, Proposition 1.1 implies that

$$
\langle\overrightarrow{A B}, \overrightarrow{E F}\rangle_{A}=\langle\overrightarrow{A A}, \overrightarrow{E F}\rangle_{A}=0 \text { and }\langle\overrightarrow{C D}, \overrightarrow{G H}\rangle_{A}=\langle\overrightarrow{C C}, \overrightarrow{G H}\rangle_{A}=0
$$

Therefore, $\langle\overrightarrow{A B}, \overrightarrow{E F}\rangle_{A}=\langle\overrightarrow{C D}, \overrightarrow{G H}\rangle_{A}=0$. A similar argument follows if $E={ }_{P} F$ (or $G={ }_{P} H$ ).

Secondly, we consider the case where $A \neq_{P} B, C \neq_{P} D, E \neq_{P} F$, and $G \neq_{P} H$. Then, none of the quantities $\|\overrightarrow{A B}\|_{A},\|\overrightarrow{C D}\|_{A},\|\overrightarrow{E F}\|_{A}$, and $\|\overrightarrow{G H}\|_{A}$ is zero. Our proof technique is to write all of the quantities that appear in Definition 10 in terms of $\overrightarrow{O P}$. Since $\overrightarrow{A B}={ }_{A} \overrightarrow{A O}+{ }_{A} \overrightarrow{O B}$, Definition 6 and Equation (1.5) imply that

$$
\begin{aligned}
\|\overrightarrow{A B}\|_{A}^{2} & =\left\|\overrightarrow{A O}+{ }_{A} \overrightarrow{O B}\right\|_{A}^{2}=\left\langle\overrightarrow{A O}+{ }_{A} \overrightarrow{O B}, \overrightarrow{A O}+{ }_{A} \overrightarrow{O B}\right\rangle_{A} \\
& =\langle\overrightarrow{A O}, \overrightarrow{A O}\rangle_{A}+2\langle\overrightarrow{A O}, \overrightarrow{O B}\rangle_{A}+\langle\overrightarrow{O B}, \overrightarrow{O B}\rangle_{A}
\end{aligned}
$$

Since $A$ and $B$ are points in $\mathscr{P}_{l}$, by Definition 8 there exist $a, b \in \mathbb{R}$ such that

$$
\overrightarrow{O A}={ }_{A}(a) \overrightarrow{O P}, \quad \overrightarrow{O B}={ }_{A}(b) \overrightarrow{O P}
$$

It follows by Definition 4 that

$$
\overrightarrow{A O}={ }_{A}-((a) \overrightarrow{O P})
$$


Now, if we substitute Equation (1.77) and the second equation from (1.76) into the right hand side of the Equation (1.75) and use Equations (1.8) and (1.6), we get

$$
\|\overrightarrow{A B}\|_{A}^{2}=\left(a^{2}-2 a b+b^{2}\right)\langle\overrightarrow{O P}, \overrightarrow{O P}\rangle_{A}=(a-b)^{2}\|\overrightarrow{O P}\|_{A}^{2}
$$

Taking the positive square root of both sides in the above yields

$$
\|\overrightarrow{A B}\|_{A}=|a-b|\|\overrightarrow{O P}\|
$$

Similarly, we can write

$$
\overrightarrow{C D}={ }_{A} \overrightarrow{C O}+{ }_{A} \overrightarrow{O D}, \overrightarrow{E F}={ }_{A} \overrightarrow{E O}+{ }_{A} \overrightarrow{O F} \text {, and } \overrightarrow{G H}={ }_{A} \overrightarrow{G O}+{ }_{A} \overrightarrow{O H}
$$

Since $\overrightarrow{C D}, \overrightarrow{E F}$, and $\overrightarrow{G H}$ are in $\mathscr{P}_{l_{A}}$ we see that the points $C, D, E, F, G$, and $H$ lie on the line $l_{O P}$. Hence, by Definition 8 there are real numbers $c, d, e, f, g$, and $h$ such that

$$
\begin{aligned}
& \overrightarrow{O C}={ }_{A}(c) \overrightarrow{O P}, \overrightarrow{O D}={ }_{A}(d) \overrightarrow{O P}, \overrightarrow{O E}={ }_{A}(e) \overrightarrow{O P} \\
& \overrightarrow{O F}={ }_{A}(f) \overrightarrow{O P}, \overrightarrow{O G}={ }_{A}(g) \overrightarrow{O P}, \overrightarrow{O H}={ }_{A}(h) \overrightarrow{O H}
\end{aligned}
$$

If we repeat the steps that led to Equations (1.75) through (1.78) we obtain

$$
\|\overrightarrow{C D}\|_{A}=|c-d|\|\overrightarrow{O P}\|,\|\overrightarrow{E F}\|_{A}=|e-f|\|\overrightarrow{O P}\|,\|\overrightarrow{G H}\|_{A}=|g-h|\|\overrightarrow{O P}\| .
$$

Calculations similar to those used to derive Equation (1.78) show that

$$
\langle\overrightarrow{A B}, \overrightarrow{E F}\rangle_{A}=(a-b)(e-f)\langle\overrightarrow{O P}, \overrightarrow{O P}\rangle_{A},\langle\overrightarrow{C D}, \overrightarrow{G H}\rangle_{A}=(c-d)(g-h)\langle\overrightarrow{O P}, \overrightarrow{O P}\rangle_{A}
$$

We want to emphasize that none of the quantities $|a-b|,|c-d|,|e-f|$, and $|g-h|$ is zero. This is because, for example, $|a-b|=0$ means $a=b$. But by the two equations in (1.76) $a=b$ would imply that $\overrightarrow{O A}={ }_{A} \overrightarrow{O B}$. If this is true, then we would have, by Definition 3, that $A={ }_{P} B$. This is a contradiction to our the assumption of $A \neq{ }_{P} B$. The same argument applies for $|c-d|,|e-f|$, and $|g-h|$.

Now, by Equations (1.78), (1.81), and (1.82), we have

$$
\frac{\langle\overrightarrow{A B}, \overrightarrow{E F}\rangle_{A}}{\|\overrightarrow{A B}\|_{A}\|\overrightarrow{E F}\|_{A}}=\frac{(a-b)(e-f)}{|a-b||e-f|}
$$

and

$$
\frac{\langle\overrightarrow{C D}, \overrightarrow{G H}\rangle_{A}}{\|\overrightarrow{C D}\|_{A}\|\overrightarrow{G H}\|_{A}}=\frac{(c-d)(g-h)}{|c-d||g-h|}
$$


In a similar manner we obtain

$$
\frac{\langle\overrightarrow{A B}, \overrightarrow{C D}\rangle_{A}}{\|\overrightarrow{A B}\|_{A}\|\overrightarrow{C D}\|_{A}}=\frac{(a-b)(c-d)}{|a-b||c-d|}
$$

and

$$
\frac{\langle\overrightarrow{E F}, \overrightarrow{G H}\rangle_{A}}{\|\overrightarrow{E F}\|_{A}\|\overrightarrow{G H}\|_{A}}=\frac{(e-f)(g-h)}{|e-f||g-h|}
$$

Since $\overrightarrow{A B} \Re_{l} \overrightarrow{C D}$, Definition 10 implies that $\frac{\langle\overrightarrow{A B}, \overrightarrow{C D}\rangle_{A}}{\|\overrightarrow{A B}\|_{A}\|\overrightarrow{C D}\|_{A}}=1$. Hence, we conclude from Equation (1.85) that $\frac{(a-b)(c-d)}{|a-b||c-d|}=1$, which means that

$$
(a-b)(c-d)>0 .
$$

A similar argument shows that

$$
(e-f)(g-h)>0 \text {. }
$$

The signs of the products $(a-b)(e-f)$ and $(c-d)(g-h)$ in Equations (1.83) and (1.84) will decide whether the right hand side of each of these two equations is 1 or -1 . We want to prove that the products $(a-b)(e-f)$ and $(c-d)(g-h)$ have the same sign. To do so, we set up the following table.

\begin{tabular}{|c|c|c|c|c|}
\hline$a-b$ & + & + & - & - \\
\hline$c-d$ & + & + & - & - \\
\hline$e-f$ & + & - & + & - \\
\hline$g-h$ & + & - & + & - \\
\hline$(a-b)(e-f)$ & + & - & - & + \\
\hline$(c-d)(g-h)$ & + & - & - & + \\
\hline
\end{tabular}

We conclude from the preceding table that the two products $(a-b)(e-f)$ and $(c-d)(g-h)$ are either both positive or negative. This means that in Equations (1.83) and (1.84) we have

$$
\frac{\langle\overrightarrow{A B}, \overrightarrow{E F}\rangle_{A}}{\|\overrightarrow{A B}\|_{A}\|\overrightarrow{E F}\|_{A}}=\frac{\langle\overrightarrow{C D}, \overrightarrow{G H}\rangle_{A}}{\|\overrightarrow{C D}\|_{A}\|\overrightarrow{G H}\|_{A}}= \pm 1
$$

Now, since $\overrightarrow{A B} \Re_{l} \overrightarrow{C D}$ and $\overrightarrow{E F} \Re_{l} \overrightarrow{G H}$, we have that $\|\overrightarrow{A B}\|_{A}=\|\overrightarrow{C D}\|_{A}$ and $\|\overrightarrow{E F}\|_{A}=$ $\|\overrightarrow{G H}\|_{A}$. Thus, Equation (1.89) simplifies to the desired conclusion, that is

$$
\langle\overrightarrow{A B}, \overrightarrow{E F}\rangle_{A}=\langle\overrightarrow{C D}, \overrightarrow{G H}\rangle_{A}
$$


With aid of Theorem 1.12 we prove the following result which will be used directly in proving that $\mathfrak{R}_{l}$ is an equivalence relation.

Theorem 1.13. Let $l_{O P}$ be a line and $\mathscr{P}_{l}$ be the set of points on this line. Let $\overrightarrow{A B}, \overrightarrow{C D}, \overrightarrow{E F}$ be three arrows in $\mathscr{P}_{l_{A}}$ such that $\overrightarrow{A B} \mathfrak{R}_{l} \overrightarrow{C D}$ and $\overrightarrow{E F} \mathfrak{R}_{l} \overrightarrow{C D}$. Then $\overrightarrow{A B} \mathfrak{R}_{l} \overrightarrow{E F}$.

Proof : First, if $A={ }_{P} B$ (similarly, if $C={ }_{P} D$ or $E={ }_{P} F$ ), then since $\overrightarrow{A B} \Re_{l} \overrightarrow{C D}$, it follows by Proposition 1.9 that $C={ }_{P} D$. Similarly, $C={ }_{P} D$ and $\overrightarrow{E F} \Re_{l} \overrightarrow{C D}$ means that $E={ }_{P} F$. We conclude that if $A={ }_{P} B$, then $E={ }_{P} F$, which means by Definition 10 that $\overrightarrow{A B} \Re_{l} \overrightarrow{E F}$.

Suppose now that $A \neq_{P} B, C \neq_{P} D$, and $E \neq_{P} F$. It follows by Lemma 1.1 that

$$
\|\overrightarrow{A B}\|_{A} \neq 0,\|\overrightarrow{C D}\|_{A} \neq 0, \text { and }\|\overrightarrow{E F}\|_{A} \neq 0 .
$$

Since $\overrightarrow{A B} \Re_{l} \overrightarrow{C D}$ and $\overrightarrow{E F} \Re_{l} \overrightarrow{C D}$, Definition 10 implies that

$$
\left\langle\frac{\overrightarrow{A B}}{\|\overrightarrow{A B}\|_{A}}, \frac{\overrightarrow{C D}}{\|\overrightarrow{C D}\|_{A}}\right\rangle_{A}=\left\langle\frac{\overrightarrow{E F}}{\|\overrightarrow{E F}\|_{A}}, \frac{\overrightarrow{C D}}{\|\overrightarrow{C D}\|_{A}}\right\rangle_{A}=1
$$

and

$$
\|\overrightarrow{A B}\|_{A}=\|\overrightarrow{C D}\|_{A}, \quad\|\overrightarrow{C D}\|_{A}=\|\overrightarrow{E F}\|_{A} .
$$

Furthermore, since $\overrightarrow{A B} \Re_{l} \overrightarrow{C D}$ and $\overrightarrow{E F} \Re_{l} \overrightarrow{C D}$, it follows by Theorem 1.12 that

$$
\langle\overrightarrow{A B}, \overrightarrow{E F}\rangle_{A}=\langle\overrightarrow{C D}, \overrightarrow{C D}\rangle_{A}=\|\overrightarrow{C D}\|_{A}^{2} \neq 0
$$

Dividing both sides in Equation (1.93) by $\|\overrightarrow{C D}\|_{A}^{2}$ yields $\frac{\langle\overrightarrow{A B}, \overrightarrow{E F}\rangle_{A}}{\|\overrightarrow{C D}\|_{A}^{2}}=1$. This is equivalent to $\frac{\langle\overrightarrow{A B}, \overrightarrow{E F}\rangle_{A}}{\|\overrightarrow{A B}\|_{A}\|\overrightarrow{E F}\|_{A}}=1$, as $\|\overrightarrow{A B}\|_{A}=\|\overrightarrow{C D}\|_{A}=\|\overrightarrow{E F}\|_{A}$ by (1.92), which means by Definition 10 that $\overrightarrow{A B} \Re_{l} \overrightarrow{E F}$.

Now, we show that $\mathfrak{R}_{l}$ is an equivalence relation.

Theorem 1.14. The relation $\mathfrak{N}_{l}$ in Definition 10 is an equivalence relation on $\mathscr{P}_{l_{A}}$.

Proof : We first show that the relation $\Re_{l}$ is reflexive, that is $\overrightarrow{A B} \Re_{l} \overrightarrow{A B}$. Let $\overrightarrow{A B} \in$ $\mathscr{P}_{l_{A}}$. If $A={ }_{P} B$, then it follows directly by Definition 6 that $\overrightarrow{A B} \Re_{l} \overrightarrow{A B}$. Now, suppose that $A \neq_{P} B$. Then, Definition 6 and Lemma 1.2 imply that

$$
\left\langle\frac{\overrightarrow{A B}}{\|\overrightarrow{A B}\|_{A}}, \frac{\overrightarrow{A B}}{\|\overrightarrow{A B}\|_{A}}\right\rangle_{A}=\left\|\frac{\overrightarrow{A B}}{\|\overrightarrow{A B}\|_{A}}\right\|_{A}^{2}=1
$$


Thus, $\overrightarrow{A B} \Re_{l} \overrightarrow{A B}$ for any $\overrightarrow{A B}$ in $\mathscr{P}_{l_{A}}$. Next, we show that $\mathfrak{R}_{l}$ is symmetric. Let $\overrightarrow{A B} \Re_{l} \overrightarrow{C D}$. If $A={ }_{P} B$ (similarly, if $C={ }_{P} D$ ), then since $\overrightarrow{A B} \mathfrak{R}_{l} \overrightarrow{C D}$, it follows by Proposition 1.9 that $C={ }_{P} D$. Now, since $C={ }_{P} D$ and $A={ }_{P} B$, it follows by Definition 10 that $\overrightarrow{C D} \Re_{l} \overrightarrow{A B}$. Now, if $A \neq_{P} B, C \neq_{P} D$, and $\overrightarrow{A B} \Re_{l} \overrightarrow{C D}$, then by Definition 10 we have

$$
\|\overrightarrow{A B}\|_{A}=\|\overrightarrow{C D}\|_{A}
$$

and

$$
\left\langle\frac{\overrightarrow{A B}}{\|\overrightarrow{A B}\|_{A}}, \frac{\overrightarrow{C D}}{\|\overrightarrow{C D}\|_{A}}\right\rangle_{A}=1
$$

But by Equation (1.2) we have

$$
\left\langle\frac{\overrightarrow{A B}}{\|\overrightarrow{A B}\|_{A}}, \frac{\overrightarrow{C D}}{\|\overrightarrow{C D}\|_{A}}\right\rangle_{A}=\left\langle\frac{\overrightarrow{C D}}{\|\overrightarrow{C D}\|_{A}}, \frac{\overrightarrow{A B}}{\|\overrightarrow{A B}\|_{A}}\right\rangle_{A}=1
$$

It follows by the Equations (1.94), (1.96), and Definition 10 that $\overrightarrow{C D} \Re_{l} \overrightarrow{A B}$ which means that $\Re_{l}$ is symmetric. Now, let $\overrightarrow{A B}, \overrightarrow{C D}, \overrightarrow{E F}$ be three arrows in $\mathscr{P}_{l_{A}}$ such that $\overrightarrow{A B} \mathfrak{R}_{l} \overrightarrow{C D}$ and $\overrightarrow{C D} \Re_{l} \overrightarrow{E F}$. The transitivity of $\mathfrak{R}_{l}$, that is $\overrightarrow{A B} \mathfrak{R}_{l} \overrightarrow{E F}$, follows immediately from the reflexivity of $\Re_{l}$ and Theorem 1.13 . Therefore, $\Re_{l}$ is an equivalence relation on $\mathscr{P}_{l_{A}}$.

\subsubsection{Existence of Parallel Arrow in $\mathscr{P}_{l_{A}}$}

Now we introduce, in an arrow space $\mathscr{P}_{l_{A}}$, the following important theorem which is the analog of the parallel axiom in Euclidean geometry.

Theorem 1.15. (Existence of a Unique Parallel Arrow) Given an arrow $\overrightarrow{A B}$ on a line $l_{A B}$ with $A \neq_{P} B$ and any point $P$ on $l_{A B}$, there exists a unique point $K$ on $l_{A B}$ (and $a$ unique point $K^{\prime}$ on $l_{A B}$ ) such that $\overrightarrow{A B} \Re_{l} \overrightarrow{P K}$ (likewise $\overrightarrow{A B} \Re_{l} \overrightarrow{K^{\prime} P}$ ). See Figure 1.13.

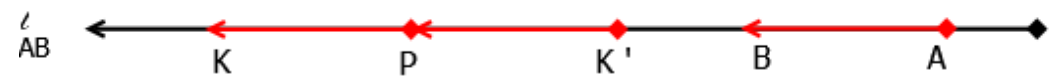

Figure 1.13: Existence of a parallel arrow.

Proof : Let $\overrightarrow{A B}$ be an arrow of $\mathscr{P}_{l_{A}}$ with $A \neq_{P} B$. Let $P \in l_{A B}$ be any point. By Definition 8 and Theorem 1.1 there exists a unique $t \in \mathbb{R}$ such that

$$
\overrightarrow{A P}={ }_{A}(t) \overrightarrow{A B}
$$


By using Definition 4 this can be rewritten as

$$
\overrightarrow{P A}={ }_{A}-((t) \overrightarrow{A B})
$$

We are looking for some real number $s \in \mathbb{R}$ and a point $K \in l_{A B}$ such that

$$
\begin{gathered}
\overrightarrow{A K}={ }_{A}(s) \overrightarrow{A B}, \\
\|\overrightarrow{A B}\|_{A}=\|\overrightarrow{P K}\|_{A},
\end{gathered}
$$

and

$$
\left\langle\frac{\overrightarrow{A B}}{\|\overrightarrow{A B}\|_{A}}, \frac{\overrightarrow{P K}}{\|\overrightarrow{P K}\|_{A}}\right\rangle_{A}=1
$$

For the fixed points $A, B, P$, Equation (1.100) indicates that we should start with $\|\overrightarrow{A B}\|_{A}=\|\overrightarrow{P K}\|_{A}$ and uniquely solve for $K$ in a manner that satisfies Equations (1.99), (1.100), and (1.101). This will involve writing all the quantities in Equation (1.100) in terms of $\overrightarrow{A B}$. Since $\overrightarrow{P K}=\overrightarrow{P A}+\overrightarrow{A K}$, we have

$$
\begin{aligned}
\|\overrightarrow{A B}\|_{A}^{2} & =\|\overrightarrow{P K}\|_{A}^{2}=\left\|\overrightarrow{P A}+{ }_{A} \overrightarrow{A K}\right\|_{A}^{2} \cdot=\left\langle\overrightarrow{P A}+{ }_{A} \overrightarrow{A K}, \overrightarrow{P A}+_{A} \overrightarrow{A K}\right\rangle_{A} \\
& =\langle\overrightarrow{P A}, \overrightarrow{P A}\rangle_{A}+2\langle\overrightarrow{P A}, \overrightarrow{A K}\rangle_{A}+\langle\overrightarrow{A K}, \overrightarrow{A K}\rangle_{A} \\
& =(t-s)^{2}\|\overrightarrow{A B}\|_{A}^{2} .
\end{aligned}
$$

Since $A \neq_{P} B$, Theorem 1.1 implies that $\|\overrightarrow{A B}\|_{A} \neq 0$. Hence, we can divide both sides in Equation (1.102) by $\|\overrightarrow{A B}\|_{A}^{2}$ to get $(t-s)^{2}=1$. Taking the square root of both sides of the later equation yields

$$
t-s= \pm 1
$$

The above equation gives us two values of $s$, namely

$$
\begin{aligned}
& s_{0}=t+1, \\
& s_{1}=t-1 .
\end{aligned}
$$

Notice that, the number $t$ is fixed by the Equation (1.97) which yields the uniqueness of $s_{0}, s_{1}$ in Equations (1.104) and (1.105). The uniqueness of $s_{0}$ and $s_{1}$, in conjunction with Axiom 3, implies the uniqueness of $K$ and $K^{\prime}$. Therefore, we express our claim as follows: there exists a unique point $K$ of $l_{A B}$ given by the equation

$$
\overrightarrow{A K}={ }_{A}\left(s_{0}\right) \overrightarrow{A B}
$$


where $s_{0}=t+1$. B construction this point satisfies Equation (1.100). It remains to confirm that this $K$ also satisfies Equation (1.101). Indeed, since $\|\overrightarrow{P K}\|_{A}=\|\overrightarrow{A B}\|_{A}$, we have

$$
\left\langle\frac{\overrightarrow{A B}}{\|\overrightarrow{A B}\|_{A}}, \frac{\overrightarrow{P K}}{\|\overrightarrow{P K}\|_{A}}\right\rangle_{A}=\left\langle\frac{\overrightarrow{A B}}{\|\overrightarrow{A B}\|_{A}}, \frac{\overrightarrow{P K}}{\|\overrightarrow{A B}\|_{A}}\right\rangle_{A}=\frac{\langle\overrightarrow{A B}, \overrightarrow{P K}\rangle_{A}}{\langle\overrightarrow{A B}, \overrightarrow{A B}\rangle_{A}}
$$

Hence, in order to prove the Equation (1.101), it is clear from the Equation (1.107) that we need only to show that

$$
\langle\overrightarrow{A B}, \overrightarrow{P K}\rangle_{A}=\langle\overrightarrow{A B}, \overrightarrow{A B}\rangle_{A}
$$

Now, since $\overrightarrow{P K}=\overrightarrow{P A}+{ }_{A} \overrightarrow{A K}$, by Equation (1.5) we have

$$
\langle\overrightarrow{A B}, \overrightarrow{P K}\rangle_{A}=\left\langle\overrightarrow{A B}, \overrightarrow{P A}+_{A} \overrightarrow{A K}\right\rangle_{A}=\langle\overrightarrow{A B}, \overrightarrow{P A}\rangle_{A}+\langle\overrightarrow{A B}, \overrightarrow{A K}\rangle_{A}
$$

Since $\overrightarrow{A K}={ }_{A}\left(s_{0}\right) \overrightarrow{A B}={ }_{A}(t+1) \overrightarrow{A B}$ and $\overrightarrow{P A}={ }_{A}-((t) \overrightarrow{A B})$, the right hand side in the above becomes

$$
\begin{aligned}
\langle\overrightarrow{A B}, \overrightarrow{P K}\rangle_{A} & =\langle\overrightarrow{A B},-((t) \overrightarrow{A B})\rangle_{A}+\langle\overrightarrow{A B},(t+1) \overrightarrow{A B}\rangle_{A} \\
& =-t\langle\overrightarrow{A B}, \overrightarrow{A B}\rangle_{A}+(t+1)\langle\overrightarrow{A B}, \overrightarrow{A B}\rangle_{A}
\end{aligned}
$$

where the final equality follows from Equation (1.8). Rearranging the above yields $\langle\overrightarrow{A B}, \overrightarrow{P K}\rangle_{A}=\langle\overrightarrow{A B}, \overrightarrow{A B}\rangle_{A}$. This means that Equation (1.108) holds and combining Equations (1.107) and (1.108) yields Equation (1.101) as desired. 


\section{Chapter 2}

\section{Turning An Arrow Space into a Vector Space}

In this chapter we consider $\mathscr{P}$ to be any infinite set of points (including a set of points of a line). All definitions, axioms, and results from Chapter 1 will be considered here unless otherwise is stated. Then, all the axioms of vector spaces, see Appendix .4, follow from the setting that we established. Also, as an application of arrow spaces, we implement these tools to solve some problems from affine geometry.

\subsection{Existence of Parallel Arrow in $\mathscr{P}_{A}$}

We proved in Section 1.4.3 that the analog of the parallel axiom holds in an arrow space $\mathscr{P}_{l_{A}}$. The main goal in this section is to prove this theorem for an arbitrary arrow space, that is, $\mathscr{P}_{A}$. We will use Theorem 2.12 to prove this theorem. We also need to introduce the following proposition in order to reach this goal.

Proposition 2.1. Let $A, B$, and $C$ be three distinct points that do not lie on the same line with $\|\overrightarrow{A B}\|_{A}=\|\overrightarrow{A C}\|_{A}$. Then, we have

$$
\|\overrightarrow{C B}\|_{A}^{2}=-2\langle\overrightarrow{A C}, \overrightarrow{C B}\rangle_{A}
$$

Proof : Using Definition 5, 6, Equations 1.6, 1.5, and 1.2, we can write

$$
\begin{aligned}
\|\overrightarrow{A B}\|_{A}^{2} & =\langle\overrightarrow{A B}, \overrightarrow{A B}\rangle_{A}=\left\langle\overrightarrow{A C}+{ }_{A} \overrightarrow{C B}, \overrightarrow{A C}+{ }_{A} \overrightarrow{C B}\right\rangle_{A} \\
& =\langle\overrightarrow{A C}, \overrightarrow{A C}\rangle_{A}+\langle\overrightarrow{C B}, \overrightarrow{C B}\rangle_{A}+2\langle\overrightarrow{A C}, \overrightarrow{C B}\rangle \\
& =\|\overrightarrow{A C}\|_{A}^{2}+\|\overrightarrow{C B}\|_{A}^{2}+2\langle\overrightarrow{A C}, \overrightarrow{C B}\rangle_{A} .
\end{aligned}
$$




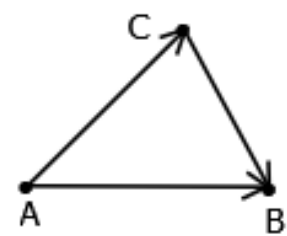

Figure 2.1: An illustration of Proposition 2.1.

Since by hypothesis $\|\overrightarrow{A B}\|_{A}=\|\overrightarrow{A C}\|_{A}$, the preceding equation simplifies to

$$
\|\overrightarrow{C B}\|_{A}^{2}=-2\langle\overrightarrow{A C}, \overrightarrow{C B}\rangle_{A},
$$

as desired.

Remark 2.1. 1. With the hypothesis of Proposition 2.1 and by following a similar argument to its proof, a similar result to Equation 2.1 holds, that is,

$$
\|\overrightarrow{C B}\|_{A}^{2}=-2\langle\overrightarrow{A B}, \overrightarrow{B C}\rangle_{A}
$$

2. Combining the preceding equation and Equation 2.1 in Proposition 2.1 yields

$$
\langle\overrightarrow{A B}, \overrightarrow{B C}\rangle_{A}=\langle\overrightarrow{A C}, \overrightarrow{C B}\rangle_{A}
$$

Then, applications of Definition 4 and Equation 1.6 to the preceding equation gives

$$
\langle\overrightarrow{B A}, \overrightarrow{B C}\rangle_{A}=\langle\overrightarrow{C A}, \overrightarrow{C B}\rangle_{A}
$$

The following result is an analog of Pythagorean Theorem in Euclidean geometry. We state and prove it in terms of arrows.

Theorem 2.1. Let $A, B$, and $C$ be three distinct points that do not lie on the same line. Then,

$$
\langle\overrightarrow{A C}, \overrightarrow{A B}\rangle_{A}=0
$$

If and only if,

$$
\|\overrightarrow{B C}\|_{A}^{2}=\|\overrightarrow{A B}\|_{A}^{2}+\|\overrightarrow{A C}\|_{A}^{2}
$$

Proof : Notice that, according to Definition 4 and Equation 1.6, we have

$$
\langle\overrightarrow{A C}, \overrightarrow{A B}\rangle_{A}=0 \text { if and only if }\langle\overrightarrow{A C}, \overrightarrow{B A}\rangle_{A}=0
$$




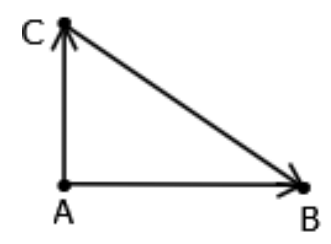

Figure 2.2: An illustration of Theorem 2.1.

Then, using Definition 5, 6, Equations 1.6, 1.5, and 1.2, we can write

$$
\begin{aligned}
\|\overrightarrow{B C}\|_{A}^{2} & =\langle\overrightarrow{B C}, \overrightarrow{B C}\rangle_{A}=\left\langle\overrightarrow{B A}+{ }_{A} \overrightarrow{A C}, \overrightarrow{B A}+{ }_{A} \overrightarrow{A C}\right\rangle_{A} \\
& =\langle\overrightarrow{B A}, \overrightarrow{B A}\rangle_{A}+\langle\overrightarrow{A C}, \overrightarrow{A C}\rangle_{A}+2\langle\overrightarrow{A C}, \overrightarrow{B A}\rangle_{A} \\
& =\|\overrightarrow{B A}\|_{A}^{2}+\|\overrightarrow{A C}\|_{A}^{2}+2\langle\overrightarrow{A C}, \overrightarrow{B A}\rangle_{A} .
\end{aligned}
$$

The preceding equation and Equation 2.6 show that $\langle\overrightarrow{A C}, \overrightarrow{A B}\rangle_{A}=0$, If and only if, $\langle\overrightarrow{A C}, \overrightarrow{B A}\rangle_{A}=0$, If and only if, $\|\overrightarrow{B C}\|_{A}^{2}=\|\overrightarrow{A B}\|_{A}^{2}+\|\overrightarrow{A C}\|_{A}^{2}$, where the equality $\|\overrightarrow{A B}\|_{A}^{2}=\|\overrightarrow{B A}\|_{A}^{2}$ follows from Equation 1.10.

Now, we can prove the existence of parallel arrow in $\mathscr{P}_{A}$.

Theorem 2.2. (The Analog of The Parallel Axiom P, Existence of a Unique Parallel Arrow) Given an arrow $\overrightarrow{A B}$ of $\mathscr{P}_{A}$ with $A \neq_{P} B$, and a point $P \in \mathscr{P}$, there exists a unique point $K$ (and a unique point $K^{\prime}$ ) in $\mathscr{P}$ such that,

$$
\|\overrightarrow{P K}\|_{A}=\|\overrightarrow{A B}\|_{A} \text { and }\left\langle\frac{\overrightarrow{A B}}{\|\overrightarrow{A B}\|_{A}}, \frac{\overrightarrow{P K}}{\|\overrightarrow{P K}\|_{A}}\right\rangle_{A}=1
$$

where $P, K$ are the tail and head of $\overrightarrow{P K}$, respectively, (likewise, $\left\|\overrightarrow{K^{\prime} P}\right\|_{A}=\|\overrightarrow{A B}\|_{A}$ $\left\langle\frac{\overrightarrow{A B}}{\|\overrightarrow{A B}\|_{A}}, \frac{\overrightarrow{K^{\prime} P}}{\left\|\overrightarrow{K^{\prime} P}\right\|_{A}}\right\rangle_{A}=1$, where $K^{\prime}, P$ are the tail and head of $\overrightarrow{K^{\prime} P}$, respectively.)

Proof : First, we show the existence part of the theorem. Consider the line $l_{A B}$ and the point $P$. Then, an application of Theorem 2.12 yields that there exists a unique point $W \in l_{A B}$ such that

$$
\langle\overrightarrow{W P}, \overrightarrow{W B}\rangle_{A}=0
$$




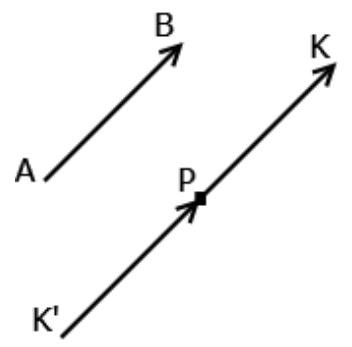

Figure 2.3: A depiction of Theorem 2.2, where $\overrightarrow{P K}$ and $\overrightarrow{K^{\prime} P}$ are equivalent to $\overrightarrow{A B}$.

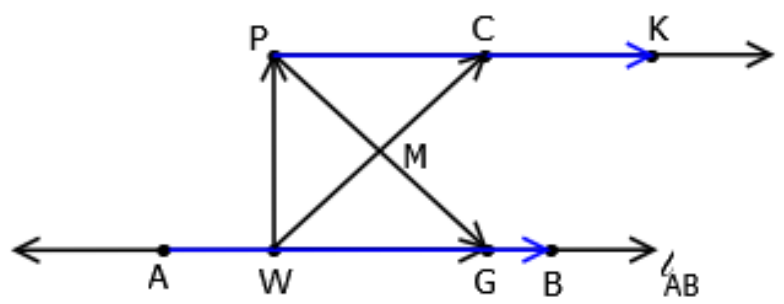

Figure 2.4: A depiction of the proof of Theorem 2.2.

Notice that, since $P \notin l_{A B}, W \in l_{A B}$, then we have $P \neq{ }_{P} W$. This means, according to Lemma 1.1, that $\|\overrightarrow{W P}\|_{A} \neq 0$. Now, let $G \in l_{A B}$ be a point given by

$$
\overrightarrow{W G}={ }_{A}(s) \overrightarrow{W B}, \text { where } s=\frac{\|\overrightarrow{W B}\|_{A}}{\|\overrightarrow{W P}\|_{A}} .
$$

Since $s$ is nonnegative, Equations 2.9 and 1.9 imply that

$$
\|\overrightarrow{W G}\|_{A}=\|(s) \overrightarrow{W B}\|_{A}=s\|\overrightarrow{W B}\|_{A}=\frac{\|\overrightarrow{W P}\|_{A}}{\|\overrightarrow{W B}\|_{A}}\|\overrightarrow{W B}\|_{A}=\|\overrightarrow{W P}\|_{A} .
$$

Notice also that since $P \notin l_{A B}, G \in l_{A B}$, then we have $P \neq_{P} G$. So, let

$$
\overrightarrow{G M}={ }_{A}\left(\frac{1}{2}\right) \overrightarrow{G P}
$$

Then, by means of Definition 8 , we have $M \in l_{G P}$ and $M \notin l_{A B}$ as $P \notin l_{A B}$. Moreover, since $P \neq{ }_{P} G$, we have by Lemma 1.1 that $M \neq{ }_{P} G$. Also, according to Definition 3 and since $\overrightarrow{G M}={ }_{A}\left(\frac{1}{2}\right) \overrightarrow{G P}$, we have that $M \neq_{P} P$. We conclude that

$$
W \neq_{P} M
$$

Thus, consider the line $l_{W M}$ and let $C$ be a point on $l_{W M}$ that is given by

$$
\overrightarrow{W C}={ }_{A}(2) \overrightarrow{W M}
$$


Since we have from the preceding argument that $M \notin l_{A B}$, it follows from the preceding equation and Definition 8 that $C \notin l_{A B}$, too.

Next, we show that $C \neq_{P} P$. Assume, by way of contradiction, that $C={ }_{P} P$. Then, Equation 2.13 would imply that

$$
\overrightarrow{W C}={ }_{A} \overrightarrow{W P}={ }_{A}(2) \overrightarrow{W M}
$$

This means, according to Definition 8, that

$$
M, W, P \in l_{W P}
$$

On the other hand, Definition 8 and Equation 2.11 imply that

$$
M, G, P \in l_{G P} \text {. }
$$

It follows by Equations 2.16, 2.15, and Theorem 1.11 that $M, G, P \in l_{W G}=l_{A B}$, which contradicts the hypothesis that $P \notin l_{A B}$. Thus, we must have $P \neq{ }_{P} C$, as desired. Now, we claim that

$$
\langle\overrightarrow{P C}, \overrightarrow{P W}\rangle_{A}=0, \text { and }\|\overrightarrow{P C}\|_{A}=\|\overrightarrow{W G}\|_{A}
$$

We first show that $\langle\overrightarrow{P C}, \overrightarrow{P W}\rangle_{A}=0$. Using Definitions 5, 6, and Equations 1.5, 1.8, and 2.13, we can write

$$
\begin{aligned}
\langle\overrightarrow{P C}, \overrightarrow{P W}\rangle_{A} & =\left\langle\overrightarrow{P W}+_{A} \overrightarrow{W C}, \overrightarrow{P W}\right\rangle_{A}=\|\overrightarrow{P W}\|_{A}^{2}+\langle\overrightarrow{W C}, \overrightarrow{P W}\rangle_{A} \\
& =\|\overrightarrow{P W}\|_{A}^{2}+2\langle\overrightarrow{W M}, \overrightarrow{P W}\rangle_{A} .
\end{aligned}
$$

Applications of Definition 5, Equation 1.5, to the term $\langle\overrightarrow{W M}, \overrightarrow{P W}\rangle_{A}$ in the right hand side of the above, yield

$$
\begin{aligned}
\langle\overrightarrow{P C}, \overrightarrow{P W}\rangle_{A} & =\|\overrightarrow{P W}\|_{A}^{2}+2\left[\langle\overrightarrow{W G}, \overrightarrow{P W}\rangle_{A}+\langle\overrightarrow{G M}, \overrightarrow{P W}\rangle_{A}\right] \\
& =\|\overrightarrow{P W}\|_{A}^{2}+2\langle\overrightarrow{G M}, \overrightarrow{P W}\rangle_{A}
\end{aligned}
$$

where

$$
\langle\overrightarrow{W G}, \overrightarrow{P W}\rangle_{A}=0
$$

follows from Equation 2.8 and Corollary 2.2. By Equations 2.11 and 1.8, the preceding equation can be rewritten as

$$
\langle\overrightarrow{P C}, \overrightarrow{P W}\rangle_{A}=\|\overrightarrow{P W}\|_{A}^{2}+2\left(\frac{1}{2}\right)\langle\overrightarrow{G P}, \overrightarrow{P W}\rangle_{A}=\|\overrightarrow{P W}\|_{A}^{2}+\langle\overrightarrow{G P}, \overrightarrow{P W}\rangle_{A}
$$


Since we have by Equation 2.10 that $\|\overrightarrow{W G}\|_{A}=\|\overrightarrow{W P}\|_{A}$, then it follows by Proposition 2.1 that the preceding equation simplifies to

$$
\langle\overrightarrow{P C}, \overrightarrow{P W}\rangle_{A}=\|\overrightarrow{P W}\|_{A}^{2}-\frac{1}{2}\|\overrightarrow{G P}\|_{A}^{2}
$$

Noticing that $\|\overrightarrow{W G}\|_{A}=\|\overrightarrow{W P}\|_{A}$, Theorem 2.1 implies that

$$
\|\overrightarrow{P W}\|_{A}^{2}=\frac{1}{2}\|\overrightarrow{G P}\|_{A}^{2}
$$

This means that Equation 2.19 is equivalent to $\langle\overrightarrow{P C}, \overrightarrow{P W}\rangle_{A}=0$, as desired.

Let's now confirm the second part of Equation 2.17, that is, we want to show that $\|\overrightarrow{P C}\|_{A}=\|\overrightarrow{W G}\|_{A}$. Notice that, $C \notin l_{W P}$ (otherwise, Equation 2.13 would imply Equation 2.15 which contradicts that fact that $P \neq{ }_{P} C$ which we just proved). Thus, the three points $W, P, C$ do not lie on the same line. Hence, since we just showed that $\langle\overrightarrow{P C}, \overrightarrow{P W}\rangle_{A}=0$, we can use Theorem 2.1 to conclude that

$$
\|\overrightarrow{W C}\|_{A}^{2}=\|\overrightarrow{W P}\|_{A}^{2}+\|\overrightarrow{C P}\|_{A}^{2}
$$

Also, Equation 2.8 and Theorem 2.1 imply that

$$
\|\overrightarrow{G P}\|_{A}^{2}=\|\overrightarrow{W G}\|_{A}^{2}+\|\overrightarrow{W P}\|_{A}^{2}
$$

If we subtract Equation 2.22 from Equation 2.21, we get

$$
\|\overrightarrow{W C}\|_{A}^{2}-\|\overrightarrow{G P}\|_{A}^{2}=\|\overrightarrow{C P}\|_{A}^{2}-\|\overrightarrow{W G}\|_{A}^{2}
$$

The preceding equation implies that to show $\|\overrightarrow{P C}\|_{A}=\|\overrightarrow{W G}\|_{A}$, it is enough to show that $\|\overrightarrow{W C}\|_{A}=\|\overrightarrow{G P}\|_{A}$. Indeed, using Definitions 5, 6, and Equation 1.8, we can write

$$
\begin{aligned}
\|\overrightarrow{W C}\|_{A}^{2}-\|\overrightarrow{G P}\|_{A}^{2} & =\langle\overrightarrow{W C}, \overrightarrow{W C}\rangle_{A}-\langle\overrightarrow{G P}, \overrightarrow{G P}\rangle_{A} \\
& =2\langle\overrightarrow{W M}, \overrightarrow{W M}\rangle_{A}-2\langle\overrightarrow{G M}, \overrightarrow{G M}\rangle_{A}
\end{aligned}
$$

The first term in the right hand sided of preceding equation can be rewritten, using Definitions 5, 6, and Equations 1.5, as follows

$$
2\langle\overrightarrow{W M}, \overrightarrow{W M}\rangle_{A}=2\left[\|\overrightarrow{W G}\|_{A}^{2}+2\langle\overrightarrow{W G}, \overrightarrow{G M}\rangle_{A}+\langle\overrightarrow{G M}, \overrightarrow{G M}\rangle_{A}\right]
$$


So, combining the preceding equation with Equation 2.24 and using Equations 2.11, 1.8 show that

$$
\begin{aligned}
\|\overrightarrow{W C}\|_{A}^{2}-\|\overrightarrow{G P}\|_{A}^{2} & =2\left[\|\overrightarrow{W G}\|_{A}^{2}+2\langle\overrightarrow{W G}, \overrightarrow{G M}\rangle_{A}\right] \\
& =2\left[\|\overrightarrow{W G}\|_{A}^{2}+2\left\langle\overrightarrow{W G},\left(\frac{1}{2}\right) \overrightarrow{G P}\right\rangle_{A}\right] \\
& =2\left[\|\overrightarrow{W G}\|_{A}^{2}+\langle\overrightarrow{W G}, \overrightarrow{G P}\rangle_{A}\right] .
\end{aligned}
$$

According to Proposition 2.1, we have that

$$
\langle\overrightarrow{W G}, \overrightarrow{G P}\rangle_{A}=-\frac{1}{2}\|\overrightarrow{G P}\|_{A}^{2}
$$

Thus, Equation 2.25 becomes

$$
\|\overrightarrow{W C}\|_{A}^{2}-\|\overrightarrow{G P}\|_{A}^{2}=2\left[\|\overrightarrow{W G}\|_{A}^{2}-\frac{1}{2}\|\overrightarrow{G P}\|_{A}^{2}\right]
$$

Since $\|\overrightarrow{W G}\|_{A}^{2}=\|\overrightarrow{P W}\|_{A}^{2}$ (by Equation 2.10), Equation 2.20 means that

$$
\|\overrightarrow{W G}\|_{A}^{2}=\frac{1}{2}\|\overrightarrow{G P}\|_{A}^{2}
$$

Therefore, Equation 2.27 simplifies to $\|\overrightarrow{W C}\|_{A}^{2}-\|\overrightarrow{G P}\|_{A}^{2}=0$. This is equivalent, by means of Equation 2.23, to $\|\overrightarrow{C P}\|_{A}^{2}-\|\overrightarrow{W G}\|_{A}^{2}=0$. That is, $\|\overrightarrow{C P}\|_{A}^{2}=\|\overrightarrow{W G}\|_{A}^{2}$. Taking the positive square root of both sides of the above and using Equation 1.10 yield

$$
\|\overrightarrow{P C}\|_{A}=\|\overrightarrow{W G}\|_{A}
$$

as desired. The next step is to show that

$$
\frac{\langle\overrightarrow{P C}, \overrightarrow{W G}\rangle_{A}}{\|\overrightarrow{P C}\|_{A}\|\overrightarrow{W G}\|_{A}}=1
$$

If we use Definitions 5, 6, and Equations 1.5, 2.18, and 2.29, we can write

$$
\frac{\langle\overrightarrow{P C}, \overrightarrow{W G}\rangle_{A}}{\|\overrightarrow{P C}\|_{A}\|\overrightarrow{W G}\|_{A}}=\frac{\langle\overrightarrow{P W}, \overrightarrow{W G}\rangle_{A}+{ }_{A}\langle\overrightarrow{W C}, \overrightarrow{W G}\rangle_{A}}{\|\overrightarrow{W G}\|_{A}^{2}}=\frac{\langle\overrightarrow{W C}, \overrightarrow{W G}\rangle_{A}}{\|\overrightarrow{W G}\|_{A}^{2}}
$$

We rewrite the numerator of the right hand side of the above, using Definitions 5, 6, and Equations 2.13, 1.5, 1.8 as follows.

$$
\begin{aligned}
\langle\overrightarrow{W C}, \overrightarrow{W G}\rangle_{A} & =2\langle\overrightarrow{W M}, \overrightarrow{W G}\rangle_{A}=2\left[\langle\overrightarrow{W G}, \overrightarrow{W G}\rangle_{A}+\langle\overrightarrow{G M}, \overrightarrow{W G}\rangle_{A}\right] \\
& =2\left[\|\left.\overrightarrow{W G}\right|_{A} ^{2}+\frac{1}{2}\langle\overrightarrow{G P}, \overrightarrow{W G}\rangle_{A}\right]=2\|\overrightarrow{W G}\|_{A}^{2}+\langle\overrightarrow{G P}, \overrightarrow{W G}\rangle_{A} .
\end{aligned}
$$


By means of Equation 2.26, the preceding equation is equivalent to

$$
\langle\overrightarrow{W C}, \overrightarrow{W G}\rangle_{A}=2\|\overrightarrow{W G}\|_{A}^{2}-\frac{1}{2}\|\overrightarrow{G P}\|_{A}^{2}
$$

Since we have by Equation 2.28 that $\|\overrightarrow{W G}\|_{A}^{2}=\frac{1}{2}\|\overrightarrow{G P}\|_{A}^{2}$, the preceding equation simplifies to

$$
\langle\overrightarrow{W C}, \overrightarrow{W G}\rangle_{A}=2\|\overrightarrow{W G}\|_{A}^{2}-\|\overrightarrow{W G}\|_{A}^{2}=\|\overrightarrow{W G}\|_{A}^{2} .
$$

If we plug in the preceding equation into Equation 2.31, we get

$$
\frac{\langle\overrightarrow{P C}, \overrightarrow{W G}\rangle_{A}}{\|\overrightarrow{P C}\|_{A}\|\overrightarrow{W G}\|_{A}}=\frac{\|\overrightarrow{W G}\|_{A}^{2}}{\|\overrightarrow{W G}\|_{A}^{2}}=1
$$

Notice that, by means of Definition 11, the preceding equation and Equation 2.29 imply that

$$
\overrightarrow{P C} \mathfrak{R} \overrightarrow{W G}
$$

Now, since $P \neq{ }_{P} C$, consider the line $l_{P C}$ and let $K$ be a point on the line $l_{P C}$ such that

$$
\overrightarrow{P K}=(t) \overrightarrow{P C}, \text { where } t=\frac{\|\overrightarrow{A B}\|_{A}}{\|\overrightarrow{W G}\|_{A}}
$$

We claim that

$$
\frac{\langle\overrightarrow{P K}, \overrightarrow{A B}\rangle_{A}}{\|\overrightarrow{P K}\|_{A}\|\overrightarrow{A B}\|_{A}}=1 \text { and }\|\overrightarrow{P K}\|_{A}=\|\overrightarrow{A B}\|_{A}
$$

Indeed, since $t=\frac{\|\overrightarrow{A B}\|_{A}}{\|\left.\overrightarrow{W G}\right|_{A}}>0$ and using Equation 1.9, we have

$$
\|\overrightarrow{P K}\|_{A}=\|(t) \overrightarrow{P C}\|_{A}=t\|\overrightarrow{P C}\|_{A}=\frac{\|\overrightarrow{A B}\|_{A}}{\|\overrightarrow{W G}\|_{A}}\|\overrightarrow{P C}\|_{A}=\|\overrightarrow{A B}\|_{A}
$$

where $\|\overrightarrow{P C}\|_{A}=\|\overrightarrow{W G}\|_{A}$ follows from Equation 2.29. To show that $\frac{\langle\overrightarrow{P K}, \overrightarrow{A B}\rangle_{A}}{\|\overrightarrow{P K}\|_{A}\|\overrightarrow{A B}\|_{A}}=1$, notice that according to Lemma 1.3 and since $W \neq_{P} G, A \neq_{P} B$, we have

$$
\frac{\langle\overrightarrow{W G}, \overrightarrow{A B}\rangle_{A}}{\|\overrightarrow{W G}\|_{A}\|\overrightarrow{A B}\|_{A}}= \pm 1 .
$$


Now, using Equations 1.8, 1.9, and 2.34 (notice that $t>0$ ), we can write

$$
\frac{\langle\overrightarrow{P K}, \overrightarrow{A B}\rangle_{A}}{\|\overrightarrow{P K}\|_{A}\|\overrightarrow{A B}\|_{A}}=\frac{t\langle\overrightarrow{P C}, \overrightarrow{A B}\rangle_{A}}{t\|\overrightarrow{P C}\|_{A}\|\overrightarrow{A B}\|_{A}}=\frac{\langle\overrightarrow{P C}, \overrightarrow{A B}\rangle_{A}}{\|\overrightarrow{P C}\|_{A}\|\overrightarrow{A B}\|_{A}} .
$$

Axiom 4 and the relation in 2.33 , we can rewrite the preceding equation as follows.

$$
\frac{\langle\overrightarrow{P K}, \overrightarrow{A B}\rangle_{A}}{\|\overrightarrow{P K}\|_{A}\|\overrightarrow{A B}\|_{A}}=\frac{\langle\overrightarrow{P C}, \overrightarrow{A B}\rangle_{A}}{\|\overrightarrow{P C}\|_{A}\|\overrightarrow{A B}\|_{A}}=\frac{\langle\overrightarrow{W G}, \overrightarrow{A B}\rangle_{A}}{\|\overrightarrow{W G}\|_{A}\|\overrightarrow{A B}\|_{A}}= \pm 1
$$

where the last equality follows from Equation 2.36. Thus, if

$$
\frac{\langle\overrightarrow{P K}, \overrightarrow{A B}\rangle_{A}}{\|\overrightarrow{P K}\|_{A}\|\overrightarrow{A B}\|_{A}}=1
$$

then we are done for the existence part. If

$$
\frac{\langle\overrightarrow{P K}, \overrightarrow{A B}\rangle_{A}}{\|\overrightarrow{P K}\|_{A}\|\overrightarrow{A B}\|_{A}}=-1
$$

then let $\overrightarrow{P K^{\prime}}=(-1) \overrightarrow{P K}$ and then by the preceding Equation, we should have

$$
\frac{\left\langle\overrightarrow{P K^{\prime}}, \overrightarrow{A B}\right\rangle_{A}}{\left\|\overrightarrow{P K^{\prime}}\right\|_{A}\|\overrightarrow{A B}\|_{A}}=\frac{-\langle\overrightarrow{P K}, \overrightarrow{A B}\rangle_{A}}{\|\overrightarrow{P K}\|_{A}\|\overrightarrow{A B}\|_{A}}=1 .
$$

Also,

$$
\left\|\overrightarrow{P K^{\prime}}\right\|_{A}=\|(-1) \overrightarrow{P K}\|_{A}=\|\overrightarrow{A B}\|_{A}
$$

where the last equality follows from Equation 2.35.

Secondly, we show that this point, that is the point $K$ is unique. Let $N$ be a point such that $\overrightarrow{P N}$ satisfies Equation 2.7 as $\overrightarrow{P K}$. Then, Proposition 1.5 would imply that $K={ }_{P} N$, as desired.

\subsection{An Equivalence Relation on Arrow Spaces}

For an arbitrary arrow space $\mathscr{P}_{A}$, we need to extend the definition of an equivalence relation on a line introduced in Section 1.4 in Chapter 1, which means restating Definition 10 in the context of an arbitrary arrow space and defining $\mathfrak{R}$. However, we 
need to make some changes to show that $\Re$ is an equivalence relation. In Section 1.4 in Chapter 1 we had all points lie on line $l_{O P}$. This restriction in direction allowed us to write all arrows as a scalar multiple of a fixed arrow $\overrightarrow{O P}$ and directly prove Theorem 1.12, a result key to proving the transitivity of $\Re_{l}$; see Theorem 1.13. However, if we try to prove the analog of Theorem 1.12 for a general arrow space we would face the difficulty expressing arrows in terms of one fixed arrow. Therefore, since this statement is crucial to prove transitivity of the relation $\Re$, we express it as an axiom, namely Axiom 4. Once $\Re$ is shown to be an equivalence relation we can supplement $\mathscr{P}_{A}$ with vector algebra and form the associated vector space $\mathscr{P}_{v}$. The construction of $\mathscr{P}_{v}$ from $\mathscr{P}_{A}$ can also be applied to $\mathscr{P}_{l_{A}}$, where Axiom 4 must be replaced by Theorems 1.12. Thus, less axioms are required in the case of line than the general case.

We start by defining a relation $\Re$ on $\mathscr{P}_{A}$.

Definition 11. Let $\overrightarrow{A B}$ and $\overrightarrow{C D}$ be two arrows in $\mathscr{P}_{A}$. We say that $\overrightarrow{A B} \Re \overrightarrow{C D}$ if and only if, either $A={ }_{P} B$ and $C={ }_{P} D$, or

1. $\|\overrightarrow{A B}\|_{A}=\|\overrightarrow{C D}\|_{A}$, and

2. $\left\langle\frac{\overrightarrow{A B}}{\|\overrightarrow{A B}\|_{A}}, \frac{\overrightarrow{C D}}{\|\overrightarrow{C D}\|_{A}}\right\rangle_{A}=1$.

We need the following axiom to prove transitivity of $\Re$.

Axiom 4. Given $\overrightarrow{A B}, \overrightarrow{C D}, \overrightarrow{E F}$, and $\overrightarrow{G H}$ such that $\overrightarrow{A B} \Re \overrightarrow{C D}$ and $\overrightarrow{E F} \Re \overrightarrow{G H}$, then

$$
\langle\overrightarrow{A B}, \overrightarrow{E F}\rangle_{A}=\langle\overrightarrow{C D}, \overrightarrow{G H}\rangle_{A}
$$
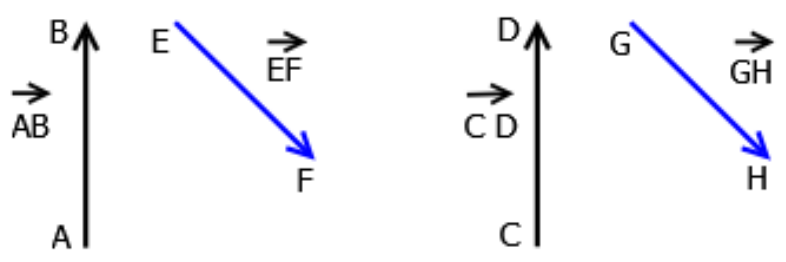

Figure 2.5: An illustration of Axiom 4 where equivalent arrows share the same color.

The proof of the following theorem is technically the same as that of Theorem 1.13, where Equation (2.39) of Axiom 4 is to be used instead of Theorem 1.12.

Theorem 2.3. Let $\overrightarrow{A B}, \overrightarrow{C D}, \overrightarrow{E F}$ be three arrows in $\mathscr{P}_{A}$ such that $\overrightarrow{A B} \Re \overrightarrow{C D}$ and $\overrightarrow{C D} \Re \overrightarrow{E F}$. Then, $\overrightarrow{A B} \Re \overrightarrow{E F}$. 
Since the proof that $\Re$ is an equivalence relation is analogous to that used to show that $\mathfrak{R}_{l}$ was a equivalence relation, we will skip the proof of the following theorem.

Theorem 2.4. The relation $\mathfrak{R}$ in Definition 11 is an equivalence relation on $\mathscr{P}_{A}$.

We are now ready to introduce vectors.

Definition 12. Consider the family of all equivalence classes that are obtained from Theorem 2.4 and denote it by $\mathscr{P}_{v}$. We call each equivalence class $[\overrightarrow{A B}] \in \mathscr{P}_{v}$ a vector and denote $[\overrightarrow{A B}]$ by $v$. In particular, if $A={ }_{P} B$, the equivalence class $[\overrightarrow{A A}]$ is the zero vector and is denoted $\overrightarrow{0}$.

Example 1. The space of points $\mathbb{R}^{n}$ viewed as a Cartesian space is of great interest in theory and applications. Since for $a, b \in \mathbb{R}$, addition $a+b$ and multiplication $a b$ are well defined, given any two points $A=\left(a_{1}, a_{2}, \cdots, a_{n}\right)$ and $B=\left(b_{1}, b_{2}, \cdots, b_{n}\right)$ in $\mathbb{R}^{n}$ and given any $s \in \mathbb{R}$, the operations

$$
A+B:=\left(a_{1}+b_{1}, a_{2}+b_{2}, \cdots, a_{n}+b_{n}\right), \quad s A:=\left(s a_{1}, s a_{2}, \cdots, s a_{n}\right)
$$

preceded the notions of a vector space with the definite set of axioms accepted today. In particular,

$$
\overrightarrow{A B}:=\left(b_{1}-a_{1}, b_{2}-a_{2}, \cdots, b_{n}-a_{n}\right) .
$$

By using the definitions of (2.40) and (2.41), given an arrow $\overrightarrow{A B}$ and a point $T=$ $\left(t_{1}, t_{2}, \cdots, t_{n}\right)$, there exists a unique point $H=\left(h_{1}, h_{2}, \cdots, h_{n}\right)$ such that $\overrightarrow{T H}$ is in the same equivalence class as $\overrightarrow{A B}$. The coordinates of $H=\left(h_{1}, h_{2}, \cdots, h_{n}\right)$ are determined by

$$
h_{j}=t_{j}+\left(b_{j}-a_{j}\right), j=1,2, \cdots, n
$$

Also for any two arbitrary arrows $\overrightarrow{A B}$ and $\overrightarrow{T H}$ in $\mathbb{R}^{n}$, the arrow pre-inner product is defined as

$$
\langle\overrightarrow{A B}, \overrightarrow{T H}\rangle_{A}:=\sum_{j=1}^{n}\left(b_{j}-a_{j}\right)\left(h_{j}-t_{j}\right) .
$$

Hence, Theorem 2.2 is superfluous and is supplanted by (2.42). Axiom 4 can then be derived as a consequence of (2.41), (2.42), and (2.43).

A comparison with the analog in affine spaces is noteworthy. Consider the Cartesian product $\mathbb{L}^{n} \times \mathbb{V}^{n}$, where $\mathbb{L}^{n}$ is viewed as a Cartesian space with elements

$$
\left(a_{1}, a_{2}, \cdots, a_{n}\right) \in \mathbb{L}^{n},
$$


and $\mathbb{V}^{n}$ is viewed as the vector space $\mathbb{V}^{n}=\mathbb{R}^{n}$ over the field $\mathbb{R}$ with elements that are vectors $v=\left\langle v_{1}, v_{2}, \cdots, v_{n}\right\rangle \in \mathbb{R}^{n}$. Then, the mapping from $\mathbb{L}^{n} \times \mathbb{V}^{n}$ into $\mathbb{L}^{n}$ is given by

$$
T+{ }_{G A} v:=H, \quad \text { where } \quad h_{j}=t_{j}+v_{j}, j=1,2, \cdots, n .
$$

Next, we use Theorem 2.2 and Definition 5 to define an addition of the equivalence classes (vector addition). We use different notations for equality and addition of equivalence classes than that of arrows, namely $=_{V}$ and $+_{V}$, respectively.

Definition 13. Let $[\overrightarrow{A B}]$ and $[\overrightarrow{C D}] \in \mathscr{P}_{v}$. Let $P$ be any point in $\mathscr{P}$. Apply Theorem 2.2 and consider the two unique arrows $\overrightarrow{K P}$ and $\overrightarrow{P L}$ such that $\overrightarrow{K P} \Re \overrightarrow{A B}$ and $\overrightarrow{P L} \Re \overrightarrow{C D}$. We define $[\overrightarrow{A B}]+{ }_{V}[\overrightarrow{C D}]:={ }_{V}\left[\overrightarrow{K P}+{ }_{A} \overrightarrow{P L}\right]={ }_{V}[\overrightarrow{K L}]$.

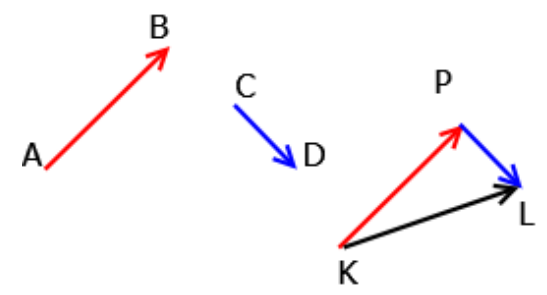

Figure 2.6: An illustration of vector addition where equivalent arrows share the same color.

Now we want to show that vector addition as given by Definition 13 is independent of the choice of the point $P$. This will be an easy task after we introduce the three following results.

Lemma 2.1. Let $\left\{P_{1}, K_{1}, L_{1}\right\}$ and $\left\{P_{2}, K_{2}, L_{2}\right\}$ be subsets of $\mathscr{P}$, and let $\overrightarrow{K_{1} P_{1}}, \overrightarrow{P_{1} L_{1}}$, $\overrightarrow{K_{2} P_{2}}$, and $\overrightarrow{P_{2} L_{2}}$ be elements of $\mathscr{P}_{A}$ be such that $\overrightarrow{K_{1} P_{1}} \Re \overrightarrow{K_{2} P_{2}}$ and $\overrightarrow{P_{1} L_{1}} \Re \overrightarrow{P_{2} L_{2}}$; see Figure 2.7. Then,

$$
\left\|\overrightarrow{K_{1} L_{1}}\right\|_{A}=\left\|\overrightarrow{K_{2} L_{2}}\right\|_{A}
$$

Proof : Since $\overrightarrow{K_{1} P_{1}} \Re \overrightarrow{K_{2} P_{2}}$ and $\overrightarrow{P_{1} L_{1}} \Re \overrightarrow{P_{2} L_{2}}$, Definition 11 implies that

$$
\left\|\overrightarrow{K_{1} P_{1}}\right\|_{A}=\left\|\overrightarrow{K_{2} P_{2}}\right\|_{A} \quad \text { and } \quad\left\|\overrightarrow{P_{1} L_{1}}\right\|_{A}=\left\|\overrightarrow{P_{2} L_{2}}\right\|_{A},
$$

which is equivalent to

$$
\left\langle\overrightarrow{K_{1} P_{1}}, \overrightarrow{K_{1} P_{1}}\right\rangle_{A}=\left\langle\overrightarrow{K_{2} P_{2}}, \overrightarrow{K_{2} P_{2}}\right\rangle_{A} \text { and }\left\langle\overrightarrow{P_{1} L_{1}}, \overrightarrow{P_{1} L_{1}}\right\rangle_{A}=\left\langle\overrightarrow{P_{2} L_{2}}, \overrightarrow{P_{2} L_{2}}\right\rangle_{A}
$$

Since $\overrightarrow{K_{1} P_{1}} \Re \overrightarrow{K_{2} P_{2}}$ and $\overrightarrow{P_{1} L_{1}} \Re \overrightarrow{P_{2} L_{2}}$ Axiom 4 and Equation (1.2) imply that

$$
\left\langle\overrightarrow{K_{1} P_{1}}, \overrightarrow{P_{1} L_{1}}\right\rangle_{A}=\left\langle\overrightarrow{K_{2} P_{2}}, \overrightarrow{P_{2} L_{2}}\right\rangle_{A}=\left\langle\overrightarrow{P_{2} L_{2}}, \overrightarrow{K_{2} P_{2}}\right\rangle_{A} .
$$



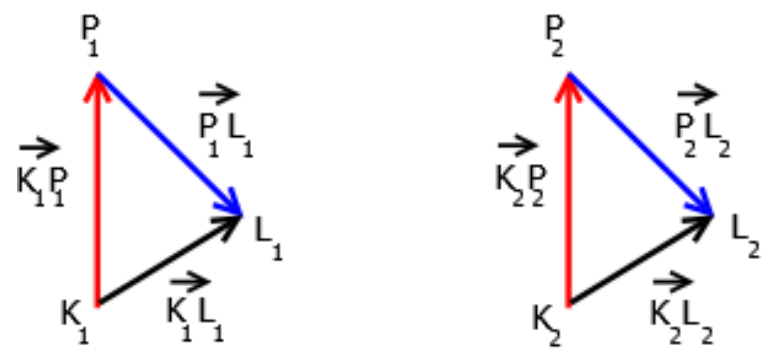

Figure 2.7: An illustration of Lemma 2.1 where equivalent arrows share the same color.

Now, by Definitions 5 and 6 and Equations (2.45), (2.46), and (1.5) we have

$$
\begin{aligned}
\left\|\overrightarrow{K_{2} L_{2}}\right\|_{A}^{2} & =\left\langle\overrightarrow{K_{2} P_{2}}+{ }_{A} \overrightarrow{P_{2} L_{2}}, \overrightarrow{K_{2} P_{2}}+{ }_{A} \overrightarrow{P_{2} L_{2}}\right\rangle_{A} \\
& =\left\langle\overrightarrow{K_{2} P_{2}}, \overrightarrow{K_{2} P_{2}}\right\rangle_{A}+2\left\langle\overrightarrow{K_{2} P_{2}}, \overrightarrow{P_{2} L_{2}}\right\rangle_{A}+\left\langle\overrightarrow{P_{2} L_{2}}, \overrightarrow{P_{2} L_{2}}\right\rangle_{A} \\
& =\left\langle\overrightarrow{K_{1} P_{1}}, \overrightarrow{K_{1} P_{1}}\right\rangle_{A}+2\left\langle\overrightarrow{K_{1} P_{1}}, \overrightarrow{P_{1} L_{1}}\right\rangle_{A}+\left\langle\overrightarrow{P_{1} L_{1}}, \overrightarrow{P_{1} L_{1}}\right\rangle_{A} \\
& =\left\langle\overrightarrow{K_{1} P_{1}}+{ }_{A} \overrightarrow{P_{1} L_{1}}, \overrightarrow{K_{1} P_{1}}+{ }_{A} \overrightarrow{P_{1} L_{1}}\right\rangle=\left\|\overrightarrow{K_{1} L_{1}}\right\|_{A}^{2} .
\end{aligned}
$$

Taking the positive square root of both sides of Equation (2.47) gives the desired result.

Theorem 2.5. Let $\left\{P_{1}, K_{1}, L_{1}\right\}$ and $\left\{P_{2}, K_{2}, L_{2}\right\}$ be subsets of $\mathscr{P}$, and let $\overrightarrow{K_{1} P_{1}}$, $\overrightarrow{P_{1} L_{1}}, \overrightarrow{K_{2} P_{2}}$, and $\overrightarrow{P_{2} L_{2}}$ be elements of $\mathscr{P}_{A}$ be such that $\overrightarrow{K_{1} P_{1}} \Re \overrightarrow{K_{2} P_{2}}$ and $\overrightarrow{P_{1} L_{1}} \Re \overrightarrow{P_{2} L_{2}}$; see Figure 2.7. Then,

$$
\frac{\left\langle\overrightarrow{K_{1} L_{1}}, \overrightarrow{K_{2} L_{2}}\right\rangle_{A}}{\left\|\overrightarrow{K_{1} L_{1}}\right\|_{A}\left\|\overrightarrow{K_{2} L_{2}}\right\|_{A}}=1 .
$$

Proof : Observe that Equation (2.48) is equivalent to

$$
\left\langle\overrightarrow{K_{1} L_{1}}, \overrightarrow{K_{2} L_{2}}\right\rangle_{A}=\left\|\overrightarrow{K_{1} L_{1}}\right\|_{A}|| \overrightarrow{K_{2} L_{2}} \|_{A} .
$$

Also we have by Lemma 2.1 that $\left\|\overrightarrow{K_{1} L_{1}}\right\|_{A}=\left\|\overrightarrow{K_{2} L_{2}}\right\|_{A}$. By using this and Definition 6, Equation (2.49) becomes

$$
\left\langle\overrightarrow{K_{1} L_{1}}, \overrightarrow{K_{2} L_{2}}\right\rangle_{A}=\left\|\overrightarrow{K_{1} L_{1}}\right\|_{A}^{2}=\left\langle\overrightarrow{K_{1} L_{1}}, \overrightarrow{K_{1} L_{1}}\right\rangle_{A} .
$$

Thus, to prove this theorem it is enough to show that Equation (2.50) holds. Now, by Definition 6 and Equation (1.5) we have 


$$
\begin{aligned}
\left\langle\overrightarrow{K_{1} L_{1}}, \overrightarrow{K_{2} L_{2}}\right\rangle_{A} & =\left\langle\overrightarrow{K_{1} P_{1}}+{ }_{A} \overrightarrow{P_{1} L_{1}}, \overrightarrow{K_{2} P_{2}}+{ }_{A} \overrightarrow{P_{2} L_{2}}\right\rangle_{A} \\
& =\left\langle\overrightarrow{K_{1} P_{1}}, \overrightarrow{K_{2} P_{2}}\right\rangle_{A}+2\left\langle\overrightarrow{K_{1} P_{1}}, \overrightarrow{P_{2} L_{2}}\right\rangle_{A}+\left\langle\overrightarrow{P_{1} L_{1}}, \overrightarrow{P_{2} L_{2}}\right\rangle_{A} .
\end{aligned}
$$

Since $\Re$ is reflexive, we have $\overrightarrow{K_{1} P_{1}} \Re \overrightarrow{K_{1} P_{1}}$. Furthermore since both $\overrightarrow{P_{1} L_{1}} \Re \overrightarrow{P_{2} L_{2}}$ and $\overrightarrow{K_{1} P_{1}} \Re \overrightarrow{K_{2} P_{2}}$, Axiom 4 implies that

$$
\left\langle\overrightarrow{K_{1} P_{1}}, \overrightarrow{P_{2} L_{2}}\right\rangle_{A}=\left\langle\overrightarrow{K_{1} P_{1}}, \overrightarrow{P_{1} L_{1}}\right\rangle_{A},
$$

and that

$$
\left\langle\overrightarrow{K_{1} P_{1}}, \overrightarrow{K_{2} P_{2}}\right\rangle=\left\langle\overrightarrow{K_{1} P_{1}}, \overrightarrow{K_{1} P_{1}}\right\rangle .
$$

Similarly, since $\overrightarrow{P_{1} L_{1}} \Re \overrightarrow{P_{1} L_{1}}$ and since both $\overrightarrow{K_{1} P_{1}} \Re \overrightarrow{K_{2} P_{2}}$ and $\overrightarrow{P_{1} L_{1}} \Re \overrightarrow{P_{2} L_{2}}$, Axiom 4 implies that

$$
\left\langle\overrightarrow{P_{1} L_{1}}, \overrightarrow{K_{2} P_{2}}\right\rangle_{A}=\left\langle\overrightarrow{P_{1} L_{1}}, \overrightarrow{K_{1} P_{1}}\right\rangle_{A}
$$

and that

$$
\left\langle\overrightarrow{P_{1} L_{1}}, \overrightarrow{P_{2} L_{2}}\right\rangle_{A}=\left\langle\overrightarrow{P_{1} L_{1}}, \overrightarrow{P_{1} L_{1}}\right\rangle_{A} .
$$

Substitution Equations (2.52) through (2.55) into the right hand side of Equation (2.51) yields

$$
\begin{aligned}
\left\langle\overrightarrow{K_{1} L_{1}}, \overrightarrow{K_{2} L_{2}}\right\rangle_{A} & =\left\langle\overrightarrow{K_{1} P_{1}}, \overrightarrow{K_{1} P_{1}}\right\rangle_{A}+2\left\langle\overrightarrow{K_{1} P_{1}}, \overrightarrow{P_{1} L_{1}}\right\rangle_{A}+\left\langle\overrightarrow{P_{1} L_{1}}, \overrightarrow{P_{1} L_{1}}\right\rangle_{A} \\
& =\left\langle\overrightarrow{K_{1} L_{1}}, \overrightarrow{K_{1} L_{1}}\right\rangle_{A},
\end{aligned}
$$

which is exactly Equation (2.50).

Corollary 2.1. Let $\left\{P_{1}, K_{1}, L_{1}\right\}$ and $\left\{P_{2}, K_{2}, L_{2}\right\}$ be subsets of $\mathscr{P}$, and let $\overrightarrow{K_{1} P_{1}}$, $\overrightarrow{P_{1} L_{1}}, \overrightarrow{K_{2} P_{2}}$, and $\overrightarrow{P_{2} L_{2}}$ be elements of $\mathscr{P}_{A}$ be such that $\overrightarrow{K_{1} P_{1}} \Re \overrightarrow{K_{2} P_{2}}$ and $\overrightarrow{P_{1} L_{1}} \Re \overrightarrow{P_{2} L_{2}}$; see Figure 2.7. Then, $\overrightarrow{K_{1} L_{1}} \Re \overrightarrow{K_{2} L_{2}}$.

Proof : By Lemma 2.1 we have

$$
\left\|\overrightarrow{K_{1} L_{1}}\right\|_{A}=\left\|\overrightarrow{K_{2} L_{2}}\right\|_{A}
$$

By Theorem 2.5 we have

$$
\frac{\left\langle\overrightarrow{K_{1} L_{1}}, \overrightarrow{K_{2} L_{2}}\right\rangle_{A}}{\left\|\vec { K _ { 1 } L _ { 1 } } \left|\left\|_{A}|| \overrightarrow{K_{2} L_{2}}\right\|_{A}\right.\right.}=1 .
$$

Equations (2.56), (2.57) imply that $\overrightarrow{K_{1} L_{1}} \Re \overrightarrow{K_{2} L_{2}}$, as desired. 
Next, we use Corollary 2.1 to prove that the addition of vectors in Definition 13 is independent from the choice of the point $P$.

Theorem 2.6. Given any two equivalence classes $[\overrightarrow{A B}],[\overrightarrow{C D}] \in \mathscr{P}_{v},[\overrightarrow{A B}]+{ }_{V}[\overrightarrow{C D}]$ is independent of the choice of the point P; see Figure 2.8.

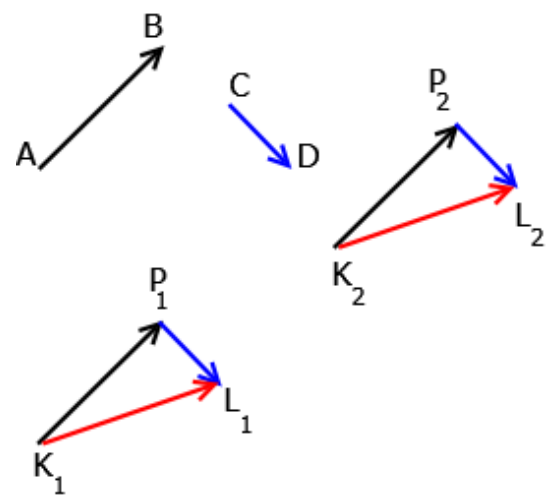

Figure 2.8: Independence of vector addition; both calculations yield the same vector addition result.

Proof : Let $[\overrightarrow{A B}],[\overrightarrow{C D}] \in \mathscr{P}_{v}$, and let $P_{1}, P_{2}$ be any two distinct points in $\mathscr{P}$. Using Theorem 2.2 let $\overrightarrow{K_{1} P_{1}}$ and $\overrightarrow{P_{1} L_{1}}$ be such that $\overrightarrow{K_{1} P_{1}} \Re \overrightarrow{A B}$ and $\overrightarrow{P_{1} L_{1}} \Re \overrightarrow{C D}$; and also let $\overrightarrow{K_{2} P_{2}}$ and $\overrightarrow{P_{2} L_{2}}$ be such that $\overrightarrow{K_{2} P_{2}} \Re \overrightarrow{A B}$ and $\overrightarrow{P_{2} L_{2}} \Re \overrightarrow{C D}$ (see Figure (2.8)). Then, since $\Re$ is transitive, it follows that

$$
\overrightarrow{K_{1} P_{1}} \Re \overrightarrow{K_{2} P_{2}} \text {, and } \overrightarrow{P_{1} L_{1}} \Re \overrightarrow{P_{2} L_{2}} \text {. }
$$

To show that Definition 13 is independent of the choice of any point, it is enough to show that $\overrightarrow{K_{1} L_{1}} \Re \overrightarrow{K_{2} L_{2}}$. This is an immediate result of Corollary 2.1 .

Now, we define vector scalar multiplication

Definition 14. Let $t \in \mathbb{R}$ and $u=[\overrightarrow{A B}]$ be any vector in $\mathscr{P}_{v}$, where $\overrightarrow{A B}$ in $\mathscr{P}_{A}$ is some representative of an equivalence class. We define the scalar multiplication

$$
t u=t[\overrightarrow{A B}]:=_{V}[(t) \overrightarrow{A B}]
$$

In order to show that Definition 14 is independent of the choice of the arrow $\overrightarrow{A B}$, we need the following lemma.

Lemma 2.2. For any two arrows $\overrightarrow{A B}, \overrightarrow{C D}$ of $\mathscr{P}_{A}$ and any $t \in \mathbb{R}$, if $\overrightarrow{A B} \Re \overrightarrow{C D}$, then $(t) \overrightarrow{A B} \Re(t) \overrightarrow{C D}$ 
Proof : If $A={ }_{P} B$, then since $\overrightarrow{A B} \Re \overrightarrow{C D}$, the analog of Proposition 1.9 implies that $C={ }_{P} D$. Then, for any $t \in \mathbb{R}$ we have by Definition 7 that

$$
(t) \overrightarrow{A B}={ }_{A}(t) \overrightarrow{A A}={ }_{A} \overrightarrow{A A}, \text { and }(t) \overrightarrow{C D}={ }_{A}(t) \overrightarrow{C C}={ }_{A} \overrightarrow{C C}
$$

Since Definition 11 implies that $\overrightarrow{A A} \Re \overrightarrow{C C}$, we conclude that $(t) \overrightarrow{A B} \Re(t) \overrightarrow{C D}$. Now, let $\overrightarrow{A B}, \overrightarrow{C D} \in \mathscr{P}_{A}$, with $A \neq_{P} B, C \neq_{P} D$, such that $\overrightarrow{A B} \Re \overrightarrow{C D}$. If $t=0$, it follows by Definition 7 that

$$
(t) \overrightarrow{A B}={ }_{A}(0) \overrightarrow{A B}={ }_{A} \overrightarrow{A A}, \text { and }(t) \overrightarrow{C D}={ }_{A}(0) \overrightarrow{C D}={ }_{A} \overrightarrow{C C}
$$

So once again we conclude that $(t) \overrightarrow{A B} \Re(t) \overrightarrow{C D}$. We now consider that $\overrightarrow{A B}, \overrightarrow{C D} \in$ $\mathscr{P}_{A}$, with $A \neq_{P} B, C \neq_{P} D$, such that $\overrightarrow{A B} \mathfrak{R} \overrightarrow{C D}$ and $t \neq 0$. Definition 11 implies that

$$
\|\overrightarrow{A B}\|_{A}=\|\overrightarrow{C D}\|_{A}
$$

and that

$$
\left\langle\frac{\overrightarrow{A B}}{\|\overrightarrow{A B}\|_{A}}, \frac{\overrightarrow{C D}}{\|\overrightarrow{C D}\|_{A}}\right\rangle_{A}=1
$$

Now, by Lemma 1.2 and Equation (2.59) we have

$$
\|(t) \overrightarrow{A B}\|_{A}=|t|\|\overrightarrow{A B}\|_{A}=|t|\|\overrightarrow{C D}\|_{A}=\|(t) \overrightarrow{C D}\|_{A}
$$

Also by Lemma 1.2, Equation (1.8), and Equation (2.60) we have

$$
\left\langle\frac{(t) \overrightarrow{A B}}{\|(t) \overrightarrow{A B}\|_{A}}, \frac{(t) \overrightarrow{C D}}{\|(t) \overrightarrow{C D}\|_{A}}\right\rangle_{A}=\left\langle\frac{(t) \overrightarrow{A B}}{|t|\|\overrightarrow{A B}\|_{A}}, \frac{(t) \overrightarrow{C D}}{|t|\|\overrightarrow{C D}\|_{A}}\right\rangle_{A}=1
$$

Combining (2.61) and (2.62) with Definition 11 shows that

$$
(t) \overrightarrow{A B} \Re(t) \overrightarrow{C D}
$$

\subsection{The Associated Vector Space}

In this section, we show that $\mathscr{P}_{v}$ with the operations of addition and scalar multiplication provided by Definitions 13 and 14 respectively, satisfy all the axioms of vector space. Definitions 13 and 14 imply that $\mathscr{P}_{v}$ is closed under these two operations. We now prove the remaining eight axioms of a vector space. We start by showing that the addition $+_{V}$ is commutative. 
Theorem 2.7. The addition $+_{v}$ is commutative, that is for any $[\overrightarrow{A B}],[\overrightarrow{C D}]$ in $\mathscr{P}_{v}$ we have

$$
[\overrightarrow{A B}]+{ }_{V}[\overrightarrow{C D}]=_{V}[\overrightarrow{C D}]+_{V}[\overrightarrow{A B}]
$$

Proof : Let $P_{1}$ be any point in $\mathscr{P}$ and use Theorem 2.2 to find the arrows $\overrightarrow{K_{1} P_{1}}$ and $\overrightarrow{P_{1} L_{1}}$ such that (see Figure (2.9) below)

$$
\overrightarrow{K_{1} P_{1}} \Re \overrightarrow{A B} \quad \text { and } \quad \overrightarrow{P_{1} L_{1}} \Re \overrightarrow{C D}
$$

Then, by Definition 13 we have

$$
[\overrightarrow{A B}]+_{V}[\overrightarrow{C D}]={ }_{V}\left[\overrightarrow{K_{1} P_{1}}+{ }_{A} \overrightarrow{P_{1} L_{1}}\right]={ }_{V}\left[\overrightarrow{K_{1} L_{1}}\right]
$$

On the other hand, let $P_{2}$ be any point and use Theorem 2.2 to find the arrows $\overrightarrow{K_{2} P_{2}}$ and $\overrightarrow{P_{2} L_{2}}$ such that (see Figure (2.9) below)

$$
\overrightarrow{P_{2} L_{2}} \Re \overrightarrow{A B} \quad \text { and } \quad \overrightarrow{K_{2} P_{2}} \Re \overrightarrow{C D}
$$

Then, we have again by Definition 13 that
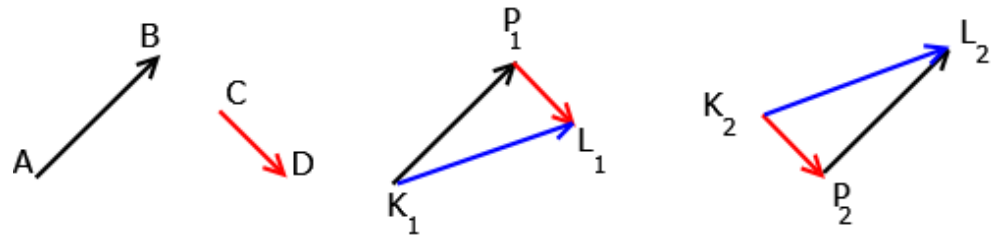

Figure 2.9: An illustration of Theorem 2.7, where equivalent arrows share the same color.

$$
[\overrightarrow{C D}]+_{V}[\overrightarrow{A B}]=_{V}\left[\overrightarrow{K_{2} P_{2}}+{ }_{A} \overrightarrow{P_{2} L_{2}}\right]={ }_{V}\left[\overrightarrow{K_{2} L_{2}}\right]
$$

Equations (2.65) and (2.67) imply that we need to prove

$$
\overrightarrow{K_{1} L_{1}} \Re \overrightarrow{K_{2} L_{2}}
$$

By Equations (2.64) and (2.66), and the transitivity of $\Re$, we have

$$
\overrightarrow{K_{1} P_{1}} \Re \overrightarrow{P_{2} L_{2}} \quad \text { and } \quad \overrightarrow{P_{1} L_{1}} \Re \overrightarrow{K_{2} P_{2}}
$$

An application of Corollary 2.1 to Equation (2.69) yields the desired result. 
We show also that the $+_{V}$ is associative and identify the additive identity as $[\overrightarrow{P P}]$ where $P$ is any point in $\mathscr{P}$.

Theorem 2.8. For any $[\overrightarrow{A B}],[\overrightarrow{C D}]$, and $[\overrightarrow{E F}]$ in $\mathscr{P}_{v}$ we have

1. the addition $+_{V}$ is associative, that is

$$
\left([\overrightarrow{A B}]+_{V}[\overrightarrow{C D}]\right)++_{V}[\overrightarrow{E F}]=_{V}[\overrightarrow{A B}]+_{V}\left([\overrightarrow{C D}]+_{V}[\overrightarrow{E F}]\right)
$$

2. for any point $P$ and any $[\overrightarrow{A B}]$ we have $[\overrightarrow{A B}]+_{V}[\overrightarrow{P P}]=_{V}[\overrightarrow{A B}]$.

Proof : (1) Let $P_{1}$ be any point in $\mathscr{P}$. By using Axiom 5, we let $\overrightarrow{K_{1} P_{1}}$ and $\overrightarrow{P_{1} L_{1}}$ be in $\mathscr{P}_{A}$ such that

$$
\overrightarrow{K_{1} P_{1}} \Re \overrightarrow{A B} \quad \text { and } \quad \overrightarrow{P_{1} L_{1}} \Re \overrightarrow{C D}
$$

Also, by an application of Theorem 2.2 to the arrow $\overrightarrow{E F}$ and the point $L_{1}$, we get the arrow $\overrightarrow{L_{1} T_{1}}$ such that

$$
\overrightarrow{L_{1} T_{1}} \Re \overrightarrow{E F}
$$

See Figure 2.10.
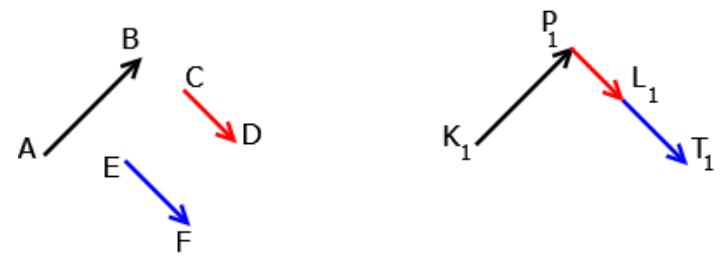

Figure 2.10: An illustration used in the proof of the associativity of vector addition. As usual, arrow equivalence is denoted via color coding.

Now, by Definition 13 and Theorem 1.4 we have

$$
\begin{aligned}
\left([\overrightarrow{A B}]+_{V}[\overrightarrow{C D}]\right)+_{V}[\overrightarrow{E F}] & ={ }_{V}\left[\overrightarrow{K_{1} P_{1}}+{ }_{A} \overrightarrow{P_{1} L_{1}}\right]+{ }_{V}\left[\overrightarrow{L_{1} T_{1}}\right] \\
& ={ }_{V}\left[\left(\overrightarrow{K_{1} P_{1}}+{ }_{A} \overrightarrow{P_{1} L_{1}}\right)+{ }_{A} \overrightarrow{L_{1} T_{1}}\right] \\
& ={ }_{V}\left[\overrightarrow{K_{1} P_{1}}+{ }_{A}\left(\overrightarrow{P_{1} L_{1}}+{ }_{A} \overrightarrow{L_{1} T_{1}}\right)\right] \\
& ={ }_{V}\left[\overrightarrow{K_{1} P_{1}}\right]+{ }_{V}\left[\overrightarrow{P_{1} L_{1}}+{ }_{A} \overrightarrow{L_{1} T_{1}}\right] \\
& ={ }_{V}[\overrightarrow{A B}]+{ }_{V}\left([\overrightarrow{C D}]+{ }_{V}[\overrightarrow{E F}]\right) .
\end{aligned}
$$

Thus, the addition $+_{V}$ is associative.

(2) Let $P$ be any point and $[\overrightarrow{A B}]$ be any given vector. By Theorem 2.2, there exists a unique arrow, say $\overrightarrow{K P}$, such that $\overrightarrow{K P} \Re \overrightarrow{A B}$. By Definition 13 we have

$$
[\overrightarrow{A B}]+_{V}[\overrightarrow{P P}]=_{V}\left[\overrightarrow{K P}+{ }_{A} \overrightarrow{P P}\right]={ }_{V}[\overrightarrow{K P}]={ }_{V}[\overrightarrow{A B}]
$$


Next, we prove that for any $[\overrightarrow{A B}]$ in $\mathscr{P}_{v}$, the additive inverse is $[-\overrightarrow{A B}]$.

Theorem 2.9. For any $[\overrightarrow{A B}]$ in $\mathscr{P}_{v}$ we have

$$
[\overrightarrow{A B}]+_{V}[-\overrightarrow{A B}]=_{V}[\overrightarrow{A A}] .
$$

Proof : By Definition 4 we have $-\overrightarrow{A B}={ }_{A} \overrightarrow{B A}$, and since $\Re$ is reflexive we have $-\overrightarrow{A B} \Re \overrightarrow{B A}$. It follows by Definitions 5 and 13 and that

$$
[\overrightarrow{A B}]+_{V}[-\overrightarrow{A B}]=_{V}[\overrightarrow{A B}]+{ }_{V}[\overrightarrow{B A}]={ }_{V}\left[\overrightarrow{A B}+{ }_{A} \overrightarrow{B A}\right]=_{V}[\overrightarrow{A A}] .
$$

In the following theorem we prove that vector scalar multiplication is associative and distributive.

Theorem 2.10. For any $[\overrightarrow{A B}]$ in $\mathscr{P}_{v}$ and $s, t \in \mathbb{R}$ we have

1. $t s[\overrightarrow{A B}]={ }_{V} t[(s) \overrightarrow{A B}]$,

2. for any arrow $\overrightarrow{A B}$ we have $(t+s)[\overrightarrow{A B}]={ }_{V} t[\overrightarrow{A B}]+{ }_{V} s[\overrightarrow{A B}]$.

Proof : (1) Let $t, s \in \mathbb{R}$ and $\overrightarrow{A B}$ be any arrow of $\mathscr{P}$. By Definition 14 we have

$$
s[\overrightarrow{A B}]={ }_{V}[(s) \overrightarrow{A B}] .
$$

An application of Definition 14 to Equation (2.71) yields

$$
\operatorname{ts}[\overrightarrow{A B}]={ }_{V} t[(s) \overrightarrow{A B}] .
$$

(2) Let $K$ and $L$ be some points that lie on the line $l_{A B}$ such that

$$
\text { (t) } \overrightarrow{A B}={ }_{A} \overrightarrow{A K} \quad \text { and } \quad(s) \overrightarrow{A B}={ }_{A} \overrightarrow{A L}
$$

By Definition 14 and Equation (2.72) we have

$$
t[\overrightarrow{A B}]+{ }_{V} s[\overrightarrow{A B}]=_{V}[(t) \overrightarrow{A B}]+_{V}[(s) \overrightarrow{A B}]=_{V}[\overrightarrow{A K}]+{ }_{V}[\overrightarrow{A L}] .
$$

If we use Theorem 2.2 for the arrow $\overrightarrow{A L}$ and the point $K$, we get the arrow $\overrightarrow{K D}$ where

$$
\overrightarrow{A L} \Re \overrightarrow{K D} \text {. }
$$

Thus, by Definition 13 we can rewrite Equation (2.73) as follows

$$
t[\overrightarrow{A B}]+{ }_{V} s[\overrightarrow{A B}]=_{V}\left[\overrightarrow{A K}+{ }_{A} \overrightarrow{K D}\right]={ }_{V}[\overrightarrow{A D}] .
$$


On the other hand, by Definition 14 we have

$$
(t+s)[\overrightarrow{A B}]=_{V}[(t+s) \overrightarrow{A B}]
$$

From Equations (2.75) and (2.76), it suffices to show that

$$
\overrightarrow{A D} \Re(t+s) \overrightarrow{A B}
$$

Various applications of Definition 6 and Equation (1.5) show that

$$
\begin{aligned}
\|\overrightarrow{A D}\|_{A}^{2} & =\langle\overrightarrow{A D}, \overrightarrow{A D}\rangle_{A}=\left\langle\overrightarrow{A K}+{ }_{A} \overrightarrow{K D}, \overrightarrow{A K}+{ }_{A} \overrightarrow{K D}\right\rangle_{A} \\
& =\langle\overrightarrow{A K}, \overrightarrow{A K}\rangle_{A}+2\langle\overrightarrow{A K}, \overrightarrow{K D}\rangle_{A}+\langle\overrightarrow{K D}, \overrightarrow{K D}\rangle_{A}
\end{aligned}
$$

Moreover, Equations (1.8) and (2.72) and Definition 6 imply that

$$
\langle\overrightarrow{A K}, \overrightarrow{A K}\rangle_{A}=\langle(t) \overrightarrow{A B},(t) \overrightarrow{A B}\rangle_{A}=t^{2}\langle\overrightarrow{A B}, \overrightarrow{A B}\rangle_{A}=t^{2}\|\overrightarrow{A B}\|_{A}^{2}
$$

Equations (2.72) and (2.74) show that

$$
\overrightarrow{K D} \Re(s) \overrightarrow{A B}
$$

and since $\mathfrak{R}$ is reflexive we have

$$
\overrightarrow{A K} \mathfrak{R} \overrightarrow{A K}
$$

Then, an application of Axiom 4 implies that

$$
\langle\overrightarrow{A K}, \overrightarrow{K D}\rangle_{A}=\langle\overrightarrow{A K},(s) \overrightarrow{A B}\rangle_{A}=\langle(t) \overrightarrow{A B},(s) \overrightarrow{A B}\rangle_{A}=t s\|\overrightarrow{A B}\|_{A}^{2}
$$

Similarly, we find that

$$
\langle\overrightarrow{K D}, \overrightarrow{K D}\rangle_{A}=s^{2}\|\overrightarrow{A B}\|_{A}^{2}
$$

If we plug the Equations (2.79), (2.82), and (2.83) into Equation (2.78), we get

$$
\|\overrightarrow{A D}\|_{A}^{2}=t^{2}\|\overrightarrow{A B}\|_{A}^{2}+2 t s\|\overrightarrow{A B}\|_{A}^{2}+s^{2}\|\overrightarrow{A B}\|_{A}^{2}=(t+s)^{2}\|\overrightarrow{A B}\|_{A}^{2}
$$

Taking the positive square root of both sides in the above yields

$$
\|\overrightarrow{A D}\|_{A}=(t+s)\|\overrightarrow{A B}\|_{A}
$$


Furthermore Equation (1.8), Lemma 1.2, and Definition 5 imply that

$$
\begin{aligned}
\frac{\langle(t+s) \overrightarrow{A B}, \overrightarrow{A D}\rangle_{A}}{\|(t+s) \overrightarrow{A B}\|_{A}\|\overrightarrow{A D}\|_{A}} & =\frac{(t+s)\langle\overrightarrow{A B}, \overrightarrow{A D}\rangle_{A}}{|t+s|\|\overrightarrow{A B}\|_{A}\|\overrightarrow{A D}\|_{A}} \\
& =\frac{(t+s)}{|t+s|} \frac{\left\langle\overrightarrow{A B}, \overrightarrow{A K}+{ }_{A} \overrightarrow{K D}\right\rangle_{A}}{\|\overrightarrow{A B}\|_{A}\|\overrightarrow{A D}\|_{A}} \\
& =\frac{(t+s)}{|t+s|}\left(\frac{\langle\overrightarrow{A B}, \overrightarrow{A K}\rangle_{A}}{\|\overrightarrow{A B}\|_{A}\|\overrightarrow{A D}\|_{A}}+\frac{\langle\overrightarrow{A B}, \overrightarrow{K D}\rangle_{A}}{\|\overrightarrow{A B}\|_{A}\|\overrightarrow{A D}\|_{A}}\right) .
\end{aligned}
$$

By definition,

$$
\langle\overrightarrow{A B}, \overrightarrow{A K}\rangle_{A}=\langle\overrightarrow{A B},(t) \overrightarrow{A B}\rangle_{A}=t\langle\overrightarrow{A B}, \overrightarrow{A B}\rangle_{A}
$$

and by an application of Axiom 4

$$
\langle\overrightarrow{A B}, \overrightarrow{K D}\rangle_{A}=\langle\overrightarrow{A B},(s) \overrightarrow{A B}\rangle_{A}=s\langle\overrightarrow{A B}, \overrightarrow{A B}\rangle_{A}
$$

If we plug Equations (2.84), (2.86), and (2.87) into Equation (2.85), we get

$$
\begin{aligned}
\frac{\langle(t+s) \overrightarrow{A B}, \overrightarrow{A D}\rangle_{A}}{\|(t+s) \overrightarrow{A B}\|_{A}\|\overrightarrow{A D}\|_{A}} & =\frac{(t+s)}{|t+s|}\left(\frac{t\langle\overrightarrow{A B}, \overrightarrow{A B}\rangle_{A}}{\|\overrightarrow{A B}\|_{A}\|\overrightarrow{A D}\|_{A}}+\frac{s\langle\overrightarrow{A B}, \overrightarrow{A B}\rangle_{A}}{\|\overrightarrow{A B}\|_{A}\|\overrightarrow{A D}\|_{A}}\right) \\
& =\frac{(t+s)^{2}}{|t+s|^{2}} \frac{\langle\overrightarrow{A B}, \overrightarrow{A B}\rangle_{A}}{\|\overrightarrow{A B}\|_{A}^{2}}=1,
\end{aligned}
$$

Equations (2.84) and (2.88) imply that $\overrightarrow{A D} \Re(t+s) \overrightarrow{A B}$ as desired.

Finally we show another distributive property of vector scalar multiplication and identify the scalar multiplicative identity as the real number 1.

Theorem 2.11. For any $[\overrightarrow{A B}],[\overrightarrow{C D}]$ in $\mathscr{P}_{v}$ and any $t \in \mathbb{R}$ we have

1. $t\left([\overrightarrow{A B}]+{ }_{V}[\overrightarrow{C D}]\right)={ }_{V} t[\overrightarrow{A B}]+{ }_{V} t[\overrightarrow{C D}]$,

2. $1[\overrightarrow{A B}]={ }_{V}[\overrightarrow{A B}]$.

Proof : (1) We first prove that

$$
t\left([\overrightarrow{A B}]+{ }_{V}[\overrightarrow{C D}]\right)=_{V} t[\overrightarrow{A B}]+{ }_{V} t[\overrightarrow{C D}]
$$


Using Theorem 2.2 for the arrow $\overrightarrow{C D}$ and the point $B$ we get the arrow $\overrightarrow{B K}$ such that $\overrightarrow{C D} \Re \overrightarrow{B K}$

Definitions 13 and 14 imply that left hand side of Equation (2.89) is

$$
t\left([\overrightarrow{A B}]+{ }_{V}[\overrightarrow{C D}]\right)=_{V} t\left[\overrightarrow{A B}+{ }_{A} \overrightarrow{B K}\right]=_{V} t[\overrightarrow{A K}]=[(t) \overrightarrow{A K}]=[\overrightarrow{A H}]
$$

for some point $H$ that lies on the line $l_{A K}$ with

$$
(t) \overrightarrow{A K} \Re \overrightarrow{A H}
$$

It follows from the preceding relation and Lemma 1.2 that

$$
|t|\|\overrightarrow{A K}\|_{A}=_{A}\|\overrightarrow{A H}\|_{A}
$$

Now, for the right hand side of the Equation (2.89) let

$$
(t) \overrightarrow{A B}={ }_{A} \overrightarrow{A M} \quad \text { and } \quad(t) \overrightarrow{C D}={ }_{A} \overrightarrow{C N}
$$

for some points $M$ and $N$ that lie on the lines $l_{A B}$ and $l_{C D}$, respectively. Then, Equation (2.89) becomes

$$
t[\overrightarrow{A B}]+_{V} t[\overrightarrow{C D}]=_{V}[(t) \overrightarrow{A B}]+_{V}[(t) \overrightarrow{C D}]=_{V}[\overrightarrow{A M}]+_{V}[\overrightarrow{C N}]
$$

Applying Theorem 2.2 to the arrow $\overrightarrow{C N}$ and the point $M$ gives the unique arrow $\overrightarrow{M L}$ such that

$$
\overrightarrow{M L} \Re \overrightarrow{C N}
$$

It follows by Definition 13 and Equations (2.94) and (2.95) that

$$
t[\overrightarrow{A B}]+_{V} t[\overrightarrow{C D}]={ }_{V}\left[\overrightarrow{A M}+{ }_{A} \overrightarrow{M L}\right]={ }_{V}[\overrightarrow{A L}]
$$

To complete the proof we need to show that

$$
\overrightarrow{A H} \Re \overrightarrow{A L}
$$

By Definitions 5 and 6 and and Equation (1.5) we have

$$
\begin{aligned}
\|\overrightarrow{A L}\|_{A}^{2} & =\langle\overrightarrow{A L}, \overrightarrow{A L}\rangle_{A}=\left\langle\overrightarrow{A M}+{ }_{A} \overrightarrow{M L}, \overrightarrow{A M}+{ }_{A} \overrightarrow{M L}\right\rangle_{A} \\
& =\langle\overrightarrow{A M}, \overrightarrow{A M}\rangle_{A}+2\langle\overrightarrow{A M}, \overrightarrow{M L}\rangle_{A}+\langle\overrightarrow{M L}, \overrightarrow{M L}\rangle_{A}
\end{aligned}
$$


Equations (1.8) and (2.93) imply that

$$
\langle\overrightarrow{A M}, \overrightarrow{A M}\rangle_{A}=\langle(t) \overrightarrow{A B},(t) \overrightarrow{A B}\rangle_{A}=t^{2}\langle\overrightarrow{A B}, \overrightarrow{A B}\rangle_{A}
$$

Since Equations (2.93) and (2.95) imply that $\overrightarrow{M L} \mathfrak{R}(t) \overrightarrow{C D}$, we also find that

$$
\langle\overrightarrow{M L}, \overrightarrow{M L}\rangle_{A}=\langle(t) \overrightarrow{C D},(t) \overrightarrow{C D}\rangle_{A}=t^{2}\langle\overrightarrow{C D}, \overrightarrow{C D}\rangle_{A}=t^{2}\langle\overrightarrow{B K}, \overrightarrow{B K}\rangle_{A},
$$

where the last equality follows by Axiom 4 and Equation (2.90). Similar calculations show that

$$
\langle\overrightarrow{A M}, \overrightarrow{M L}\rangle_{A}=t^{2}\langle\overrightarrow{A B}, \overrightarrow{C D}\rangle_{A}=t^{2}\langle\overrightarrow{A B}, \overrightarrow{B K}\rangle_{A}=t^{2}\langle\overrightarrow{B K}, \overrightarrow{A B}\rangle_{A}
$$

If we plug Equations (2.99), (2.100), and (2.101) into Equation (2.98) and use (1.5), we get

$$
\begin{aligned}
\|\overrightarrow{A L}\|_{A}^{2} & =t^{2}\langle\overrightarrow{A B}, \overrightarrow{A B}\rangle_{A}+2 t^{2}\langle\overrightarrow{A B}, \overrightarrow{B K}\rangle_{A}+t^{2}\langle\overrightarrow{B K}, \overrightarrow{B K}\rangle_{A} \\
& =t^{2}\left\langle\overrightarrow{A B}+{ }_{A} \overrightarrow{B K}, \overrightarrow{A B}+{ }_{A} \overrightarrow{B K}\right\rangle_{A}=t^{2}\langle\overrightarrow{A K}, \overrightarrow{A K}\rangle_{A}=t^{2}\|\overrightarrow{A K}\|_{A}^{2}
\end{aligned}
$$

Taking the positive square root yields $\|\overrightarrow{A L}\|_{A}=|t|\|\overrightarrow{A K}\|_{A}$. We conclude from the preceding calculation and Equation (2.92) that

$$
|t|\|\overrightarrow{A K}\|_{A}={ }_{A}\|\overrightarrow{A H}\|_{A}=\|\overrightarrow{A L}\|_{A}
$$

Now, by the Equations (1.8), (2.91), and (2.102), we have

$$
\frac{\langle\overrightarrow{A H}, \overrightarrow{A L}\rangle_{A}}{\|\overrightarrow{A H}\|_{A}\|\overrightarrow{A L}\|_{A}}=\frac{\langle(t) \overrightarrow{A K}, \overrightarrow{A L}\rangle_{A}}{|t|^{2}\|\overrightarrow{A K}\|_{A}^{2}}=\frac{t \overrightarrow{A K}, \overrightarrow{A L}\rangle_{A}}{|t|^{2}\|\overrightarrow{A K}\|_{A}^{2}}
$$

To simplify the numerator of the right term in Equation (2.103) we use Definition 5 and Equation (1.5) as follows.

$$
\begin{aligned}
\langle\overrightarrow{A K}, \overrightarrow{A L}\rangle_{A} & =\left\langle\overrightarrow{A B}+_{A} \overrightarrow{B K}, \overrightarrow{A M}+{ }_{A} \overrightarrow{M L}\right\rangle_{A} \\
& =\langle\overrightarrow{A B}, \overrightarrow{A M}\rangle_{A}+\langle\overrightarrow{A B}, \overrightarrow{M L}\rangle_{A}+\langle\overrightarrow{B K}, \overrightarrow{A M}\rangle_{A}+\langle\overrightarrow{B K}, \overrightarrow{M L}\rangle_{A}
\end{aligned}
$$

By using Equations (1.8) and (2.93), we can write

$$
\langle\overrightarrow{A B}, \overrightarrow{A M}\rangle_{A}=\langle\overrightarrow{A B},(t) \overrightarrow{A B}\rangle_{A}=t\langle\overrightarrow{A B}, \overrightarrow{A B}\rangle_{A}
$$


Since Equations (2.93) and (2.95) imply that $\overrightarrow{M L} \Re(t) \overrightarrow{C D}$, an application of Axiom 4 shows that

$$
\langle\overrightarrow{A B}, \overrightarrow{M L}\rangle_{A}=\langle\overrightarrow{A B},(t) \overrightarrow{C D}\rangle_{A}
$$

Furthermore, by Equation (2.90) we have $\overrightarrow{C D} \Re \overrightarrow{R K}$. Then, it follows by Lemma 2.2 that

$$
(t) \overrightarrow{C D} \Re(t) \overrightarrow{B K}
$$

Thus, Equation (2.106) is equivalent, to

$$
\langle\overrightarrow{A B}, \overrightarrow{M L}\rangle_{A}=\langle\overrightarrow{A B},(t) \overrightarrow{B K}\rangle_{A}=t\langle\overrightarrow{A B}, \overrightarrow{B K}\rangle_{A}
$$

Similarly, we may derive that

$$
\langle\overrightarrow{B K}, \overrightarrow{M L}\rangle_{A}=t\langle\overrightarrow{B K}, \overrightarrow{B K}\rangle_{A}
$$

Also, using Equation (1.8) and noticing that $(t) \overrightarrow{A B}={ }_{A} \overrightarrow{A M}$, we can write

$$
\langle\overrightarrow{B K}, \overrightarrow{A M}\rangle_{A}=\langle\overrightarrow{B K},(t) \overrightarrow{A B}\rangle_{A}=t\langle\overrightarrow{B K}, \overrightarrow{A B}\rangle_{A}
$$

Plugging in Equations (2.105), (2.108), (2.109), and (2.110) into Equation (2.104), yields

$$
\langle\overrightarrow{A K}, \overrightarrow{A L}\rangle_{A}=t\left(\langle\overrightarrow{A B}, \overrightarrow{A B}\rangle_{A}+\langle\overrightarrow{A B}, \overrightarrow{B K}\rangle_{A}+\langle\overrightarrow{B K}, \overrightarrow{A B}\rangle_{A}+\langle\overrightarrow{B K}, \overrightarrow{B K}\rangle_{A}\right)
$$

This can be simplified (via Definitions 5 and 6 and Equation (1.5)) to

$$
\langle\overrightarrow{A K}, \overrightarrow{A L}\rangle_{A}=t\|\overrightarrow{A K}\|_{A}^{2}
$$

Now, by Equations (2.103) and (2.111) we obtain

$$
\frac{\langle\overrightarrow{A H}, \overrightarrow{A L}\rangle_{A}}{\|\overrightarrow{A H}\|_{A}\|\overrightarrow{A L}\|_{A}}=\frac{t\langle\overrightarrow{A K}, \overrightarrow{A L}\rangle_{A}}{|t|^{2}\|\overrightarrow{A K}\|_{A}^{2}}=\frac{t^{2}\|\overrightarrow{A K}\|_{A}^{2}}{|t|^{2}\|\overrightarrow{A K}\|_{A}^{2}}=1 .
$$

Equations (2.102) and (2.112) imply that $\overrightarrow{A H} \Re \overrightarrow{A L}$ which means that Equation (2.97) holds as desired.

(2) We show that for any $[\overrightarrow{A B}]$ in $\mathscr{P}_{v}$ we have

$$
1[\overrightarrow{A B}]={ }_{V}[\overrightarrow{A B}]
$$

Now, by Definition 14 we have $1[\overrightarrow{A B}]={ }_{V}[(1) \overrightarrow{A B}]$. Thus, in order to prove Equation (2.113), we need to show that $\overrightarrow{A B} \Re(1) \overrightarrow{A B}$. By Theorem 1.8 we have (1) $\overrightarrow{A B}={ }_{A} \overrightarrow{A B}$, and since $\Re$ is reflexive, it follows that $\overrightarrow{A B} \Re(1) \overrightarrow{A B}$. 
Remark 2.2. Axioms 1, 2, and 4 show that $\langle-,-\rangle_{A}$ induces an inner product $\langle-,-\rangle_{V}$ on $\mathscr{P}_{\text {v }}$, where

$$
\langle[\overrightarrow{A B}],[\overrightarrow{C D}]\rangle_{V}:=\langle\overrightarrow{A B}, \overrightarrow{C D}\rangle_{A}
$$

\subsection{Applications of Arrow Spaces to Affine Geometry}

We close this chapter by showing how the structure of an arrow space provides a useful approach to affine geometry by solving two particular problems using the constructions that we have built so far. We start with the following theorem which defines the existence of a projection of a given point onto a given line.

Theorem 2.12. Let $O$ and $G$ be two distinct points of $\mathscr{P}$. Let $l_{O G}$ be the line containing $O$ and $G$. Given any point $P \notin l_{O G}$, there exists a unique point $W \in l_{O G}$ such that

$$
\langle\overrightarrow{W O}, \overrightarrow{W P}\rangle_{A}=0 .
$$
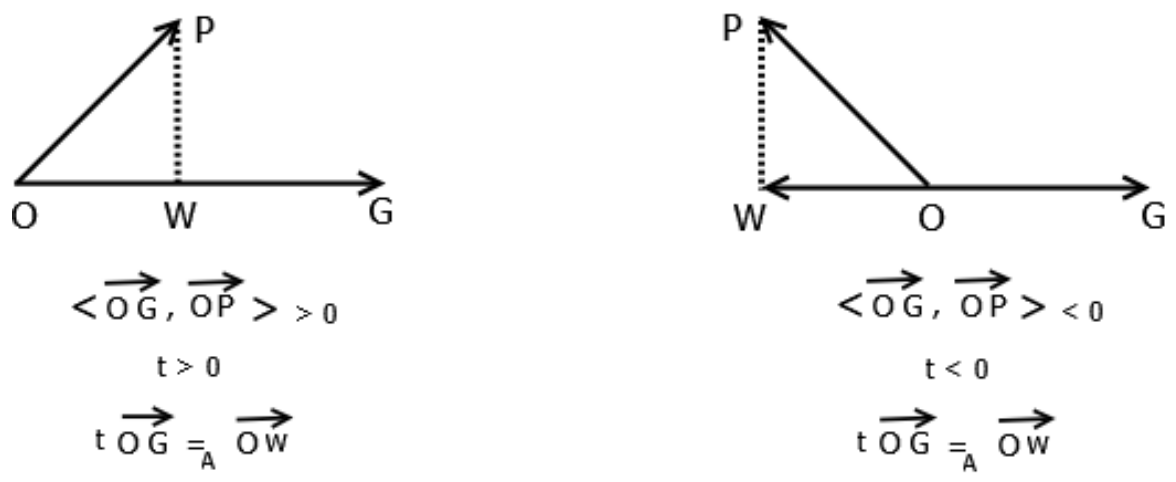

Figure 2.11: An illustration of Theorem 2.12, the existence of a point $W$ where $\langle\overrightarrow{W O}, \overrightarrow{W P}\rangle_{A}=0$.

Proof : Let $O$ and $G$ be two distinct points of $\mathscr{P}$. Let $P$ be any point of $\mathscr{P}$ such that $P \notin l_{O G}$. We want to find $t \in \mathbb{R}$ where

$$
\text { (t) } \overrightarrow{O G}={ }_{A} \overrightarrow{O W},
$$

for some $W \in l_{O G}$ which satisfies Equation (2.114); see Figure (2.11). By Definition 4 we can rewrite Equation (2.115) as

$$
-((t) \overrightarrow{O G})={ }_{A} \overrightarrow{W O}
$$


This means that Equation (2.114) is equivalent to

$$
\langle-((t) \overrightarrow{O G}), \overrightarrow{W P}\rangle_{A}=0
$$

Notice that if $t=0$, then it follows by Definition 7 and Proposition 1.1 that $O={ }_{P} W$ and the preceding equation holds trivially. For $t \neq 0$, we have by Definition 5 and Equations (1.8), (1.5), and (2.116) that

$$
\begin{aligned}
0 & =\langle-((t) \overrightarrow{O G}), \overrightarrow{W P}\rangle_{A}=-t\left\langle\overrightarrow{O G}, \overrightarrow{W O}+_{A} \overrightarrow{O P}\right\rangle_{A} \\
& =-t\langle\overrightarrow{O G}, \overrightarrow{W O}\rangle_{A}-t\langle\overrightarrow{O G}, \overrightarrow{O P}\rangle_{A} \\
& =-t\langle\overrightarrow{O G},-((t) \overrightarrow{O G})\rangle_{A}-t\langle\overrightarrow{O G}, \overrightarrow{O P}\rangle_{A} \\
& =t^{2}\langle\overrightarrow{O G}, \overrightarrow{O G}\rangle_{A}-t\langle\overrightarrow{O G}, \overrightarrow{O P}\rangle_{A}=0 .
\end{aligned}
$$

Solving the preceding equation for $t$ yields

$$
t=\frac{\langle\overrightarrow{O G}, \overrightarrow{O P}\rangle_{A}}{\langle\overrightarrow{O G}, \overrightarrow{O G}\rangle_{A}} .
$$

The two quantities $\langle\overrightarrow{O G}, \overrightarrow{O P}\rangle_{A}$ and $\langle\overrightarrow{O G}, \overrightarrow{O G}\rangle_{A}$ are uniquely determined as $O, G$, and $P$ are fixed. Hence, the $t$ in Equation (2.118) is unique. Therefore, Equation (2.115) implies that there exists a unique point $W \in l_{O G}$ such that Equation (2.114) holds.

The Equation (2.114) can be extended to hold for any arrow $\overrightarrow{W P}$ where $P \in l_{O G}$.

Corollary 2.2. Let $l_{O G}$ be a line and $P$ be any point with $P \notin l_{O G}$. Let $W \in l_{O G}$ such that

$$
\langle\overrightarrow{W O}, \overrightarrow{W P}\rangle_{A}=0
$$

Then, for any $B \in l_{O G}$ we have

$$
\langle\overrightarrow{W B}, \overrightarrow{W P}\rangle_{A}=0
$$

Proof : If $B={ }_{P} W$ or $B={ }_{P} O$, then Equation (2.120) holds as we have by Equation (2.119) that $\langle\overrightarrow{W O}, \overrightarrow{W P}\rangle_{A}=0$. Now, assume that $B \neq{ }_{P} W$ and $B \neq{ }_{P} O$. Since $W \in l_{O G}$ then it follows by Theorem 1.11 that $l_{O G}$ and $l_{O W}$ are the same line. Thus, since $B \in l_{O G}$ it is also true that $B \in l_{W O}$. This means by Definition 8 that there exists $t \neq 0$ 
(as $B \neq_{P} W$ and $B \neq_{P} O$ ) such that $\overrightarrow{W B}={ }_{A}(t) \overrightarrow{W O}$. Now it follows by the preceding equation, Equation (1.6), and Equation (2.119) that

$$
\begin{aligned}
\langle\overrightarrow{W B}, \overrightarrow{W P}\rangle_{A} & =\langle(t) \overrightarrow{W O}, \overrightarrow{W P}\rangle_{A} \\
& =t\langle\overrightarrow{W O}, \overrightarrow{W P}\rangle_{A} \\
& =t(0)=0,
\end{aligned}
$$

which ends the proof of this corollary.

Next, we define the barycentric coordinates of a point $M$ in $\mathscr{P}$, a concept crucial to the definition of affine maps (see Definitions 2.2 and 2.6 of [17]). To do so we fix an origin $O$ in $\mathscr{P}$ and introduce the following definition

Definition 15. Let $\left(P_{i}\right)_{i=1}^{i=n}$ be a fixed sequence of points in $\mathscr{P}$ and let $\left(\lambda_{i}\right)_{i=1}^{n}$ be a fixed sequence of scalars with $\sum_{i=1}^{i=n} \lambda_{i}=1$. Let $O$ be a given origin of $\mathscr{P}$. Let $M$ be a point of $\mathscr{P}$ such that $[\overrightarrow{O M}]={ }_{V} \sum_{i=1}^{i=n} \lambda_{i}\left[\overrightarrow{O P}_{i}\right]$. The real numbers $\lambda_{i} \geq 0, i=1, \ldots, n$, the barycentric coordinates of a point $M$, and $M$ is the barycenter of $\left\{P_{i}\right\}_{i=1}^{i=n}$.

To show that Definition 15 is well defined and independent of the chosen origin, we need the following theorem.

Theorem 2.13. Let $\left\{P_{i}\right\}_{i=1}^{n}$ be a family of distinct points in $\mathscr{P}$. Let $O$ be a coordinate free origin and $\left\{\lambda_{i}\right\}_{i=1}^{n}$ be a family of real numbers such that $\sum_{i=1}^{n} \lambda_{i}=1$. There exists a unique point $M$ of $\mathscr{P}$ such that $\sum_{i=1}^{n}\left[\left(\lambda_{i}\right) \overrightarrow{O P}_{i}\right]={ }_{V}[\overrightarrow{O M}]$. Moreover, the point $M$ is independent from the choice of $O$. That is, if $\tilde{O}$ is any other point and if

$$
\sum_{i=1}^{n}\left[\left(\lambda_{i}\right) \overrightarrow{\tilde{O} P_{i}}\right]=_{V}[\overrightarrow{\tilde{O} L}]
$$

then we must have $L={ }_{P}$ M. See Figure 2.12.

Proof : Let $\left(\lambda_{i}\right) \overrightarrow{O P}_{i}={ }_{A} \overrightarrow{O Q_{i}}, 1 \leq i \leq n$, for some points $\left\{Q_{i}\right\}_{i=1}^{n}$. The existence and uniqueness of a point $M$ such that

$$
\sum_{i=1}^{n}\left[\left(\lambda_{i}\right) \overrightarrow{O P}_{i}\right]={ }_{V} \sum_{i=1}^{n}\left(\lambda_{i}\right)\left[\overrightarrow{O P}_{i}\right]={ }_{V} \sum_{i=1}^{n}\left[\overrightarrow{O Q_{i}}\right]=_{V}[\overrightarrow{O M}]
$$

follows from $n-1$ applications Definition 13 as follows

$$
(n-1) \text { times }\left\{\begin{array}{l}
{\left[\overrightarrow{O Q_{1}}\right]+_{V}\left[\overrightarrow{O Q_{2}}\right]={ }_{V}\left[\overrightarrow{O Q_{1}}+_{A} \overrightarrow{Q_{1} R_{1}}\right]={ }_{V}\left[\overrightarrow{O R_{1}}\right],{ }_{V}\left[\overrightarrow{O Q_{3}}\right]={ }_{V}\left[\overrightarrow{O R_{1}}+_{A} \overrightarrow{R_{1} R_{2}}\right]={ }_{V}\left[\overrightarrow{O R_{2}}\right],} \\
: \\
{\left[\overrightarrow{O R_{n-2}}\right]+_{V}\left[\overrightarrow{O Q_{n}}\right]=_{V}\left[\overrightarrow{O R_{n-2}}+{ }_{A} \overrightarrow{R_{n-2} M}\right]=_{V}[\overrightarrow{O M}],}
\end{array}\right.
$$


where $\left\{R_{i}\right\}_{i=1}^{n-1}$ (we put $M=R_{n-1}$ ) are the unique points that we get when we apply Theorem 2.2 in Definition 13; see Figure 2.12.

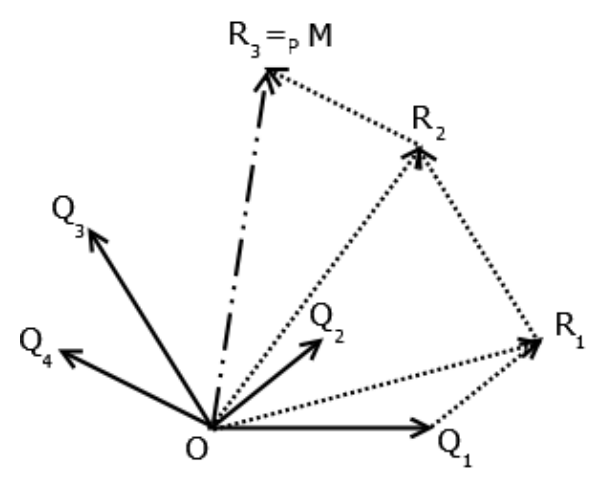

Figure 2.12: $\sum_{i=1}^{n}\left[\left(\lambda_{i}\right) \overrightarrow{O P}_{i}\right]={ }_{V} \sum_{i=1}^{n}\left[\overrightarrow{O Q_{i}}\right]={ }_{V}[\overrightarrow{O M}], n=4$.

To show that $M$ is independent of the choice of $O$ notice that by Definitions 5 and Theorem 2.11(1) we have

$$
[\overrightarrow{O M}]={ }_{V} \sum_{i=1}^{n} \lambda_{i}\left[\overrightarrow{O P}_{i}\right]={ }_{V} \sum_{i=1}^{n} \lambda_{i}\left[\overrightarrow{O \tilde{O}}+_{A} \overrightarrow{\tilde{O P}}\right]={ }_{V}[\overrightarrow{O O}] \sum_{i=1}^{n} \lambda_{i}+{ }_{V} \sum_{i=1}^{n} \lambda_{i}\left[\overrightarrow{\tilde{O} P_{i}}\right]
$$

for any point $\tilde{O}$ other than $O$. Since $\sum_{i=1}^{n} \lambda_{i}=1$, by Theorem 2.10(2) and Equations (2.122) and (2.123) we find that

$$
[\overrightarrow{O M}]={ }_{V}[\overrightarrow{O \tilde{O}}]+_{V}[\overrightarrow{\tilde{O} L}]={ }_{V}\left[\overrightarrow{O \tilde{O}}+{ }_{A} \overrightarrow{\tilde{O L}}\right]={ }_{V}[\overrightarrow{O L}]
$$

where $[\overrightarrow{\tilde{O L}}]={ }_{V} \sum_{i=1}^{n}\left(\lambda_{i}\right)\left[\overrightarrow{\tilde{O} P_{i}}\right]$ for some point $L$. This means that $\overrightarrow{O L} \Re \overrightarrow{O M}$ which implies by Proposition 1.5 that $L={ }_{P} M$, as desired. 


\section{Chapter 3}

\section{Euclidean Geometry in Arrow Spaces}

We have seen in Section 2.4 how arrow spaces can be utilized to do affine geometry. In this chapter, we shift our focus to Euclidean plane geometry. We already established the existence of a unique line that contains two given distinct points (Theorem 1.11) and the existence of a unique parallel arrow ( Theorem 2.2). Keeping in mind the definitions and results from previous chapters, we will fully prove Hilbert's axioms of Euclidean plane geometry in arrow spaces.

\subsection{Betweenness properties In Arrow Spaces}

It is well known that Hilbert provided four axioms of betweenness in order to make Euclid's work more rigorous, see [20] page 73. In this section, we prove three of the betweenness axioms in arrow spaces. The fourth is Pasch's axiom and is dealt with in Section 3.3, see Theorem 3.1. First, we provide an alternative formula for Definition 9 of betweenness in arrow spaces which also stands as an analog to the first axiom of betweenness.

Theorem 3.1. (The Axiom Bl of Betweenness) Let $A$ and $B$ be any two distinct points. Then, for any $t \in(0,1)$ such that $(t) \overrightarrow{A B}={ }_{A} \overrightarrow{A C}, C$ must be between $A$ and $B$, that is, $A \prec C \prec B$.

Proof : Let $A \neq_{P} B$ and $C$ be any points such that $(t) \overrightarrow{A B}={ }_{A} \overrightarrow{A C}$ for some $t \in(0,1)$. The preceding equation is equivalent, by Definition 4 , to

$$
-((t) \overrightarrow{A B})={ }_{A} \overrightarrow{C A}
$$

We need to show that $C$ is between $A$ and $B$, that is, we want to show (by Definition 9) that

$$
\frac{\langle\overrightarrow{C A}, \overrightarrow{C B}\rangle_{A}}{\|\overrightarrow{C A}\|_{A}\|\overrightarrow{C B}\|_{A}}=-1
$$


Now, by Definition 5, Lemma 1.9, and Equations (1.5) and (3.1) we have (notice that $t>0)$

$$
\begin{aligned}
\frac{\langle\overrightarrow{C A}, \overrightarrow{C B}\rangle_{A}}{\|\overrightarrow{C A}\|_{A}\|\overrightarrow{C B}\|_{A}} & =\frac{\left\langle\overrightarrow{C A}, \overrightarrow{C A}+_{A} \overrightarrow{A B}\right\rangle_{A}}{\|\overrightarrow{C A}\|_{A}\|\overrightarrow{C B}\|_{A}}=\frac{\langle\overrightarrow{C A}, \overrightarrow{C A}\rangle_{A}+\langle\overrightarrow{C A}, \overrightarrow{A B}\rangle_{A}}{\|\overrightarrow{C A}\|_{A}\|\overrightarrow{C B}\|_{A}} \\
& =\frac{\langle-((t) \overrightarrow{A B}),-((t) \overrightarrow{A B})\rangle_{A}}{\|-((t) \overrightarrow{A B})\|_{A}\|\overrightarrow{C B}\|_{A}}+\frac{\langle-((t) \overrightarrow{A B}), \overrightarrow{A B}\rangle_{A}}{\|-((t) \overrightarrow{A B})\|_{A}\|\overrightarrow{C B}\|_{A}} \\
& =\frac{t^{2}\langle\overrightarrow{A B}, \overrightarrow{A B}\rangle_{A}}{t\|\overrightarrow{A B}\|_{A}\|\overrightarrow{C B}\|_{A}}-\frac{t\langle\overrightarrow{A B}, \overrightarrow{A B}\rangle_{A}}{t\|\overrightarrow{A B}\|_{A}\|\overrightarrow{C B}\|_{A}} \\
& =\frac{t\|\overrightarrow{A B}\|_{A}-\|\overrightarrow{A B}\|_{A}}{\|\overrightarrow{C B}\|_{A}}=(t-1) \sqrt{\frac{\langle\overrightarrow{A B}, \overrightarrow{A B}\rangle_{A}}{\langle\overrightarrow{C B}, \overrightarrow{C B}\rangle_{A}}}
\end{aligned}
$$

Rearranging the denominator of the right hand side of the above using Definition 5 and 6, Equations (1.2), (1.5), and (3.1) again yields

$$
\begin{aligned}
\langle\overrightarrow{C B}, \overrightarrow{C B}\rangle_{A} & =\left\langle\overrightarrow{C A}+_{A} \overrightarrow{A B}, \overrightarrow{C A}+{ }_{A} \overrightarrow{A B}\right\rangle_{A} \\
& =\|\overrightarrow{C A}\|_{A}+2\langle\overrightarrow{C A}, \overrightarrow{A B}\rangle_{A}+\|\overrightarrow{A B}\|_{A}^{2} \\
& =t^{2}\|\overrightarrow{A B}\|_{A}^{2}-2 t\langle\overrightarrow{A B}, \overrightarrow{A B}\rangle_{A}+\|\overrightarrow{A B}\|_{A}^{2} \\
& =\|\overrightarrow{A B}\|_{A}^{2}\left(t^{2}-2 t+1\right) .
\end{aligned}
$$

If we plug in the preceding equation into the right hand side of Equation (3.3) noticing that $0<t<1$, we get

$$
\begin{aligned}
\frac{\langle\overrightarrow{C A}, \overrightarrow{C B}\rangle_{A}}{\|\overrightarrow{C A}\|_{A}\|\overrightarrow{C B}\|_{A}} & =(t-1) \sqrt{\frac{\|\overrightarrow{A B}\|_{A}^{2}}{\|\overrightarrow{A B}\|_{A}^{2}\left(t^{2}-2 t+1\right)}}=\frac{t-1}{\sqrt{(t-1)^{2}}} \\
& =\frac{t-1}{|t-1|}=-1 .
\end{aligned}
$$

This means that Equation (3.2) holds ending the proof of this Theorem.

Now, we prove the converse of the preceding Theorem.

Theorem 3.2. Let $A, B$, and $C$ be three distinct points such that $A \prec C \prec B$. Then, there exists a unique real number $t \in(0,1)$ such that $(t) \overrightarrow{A B}={ }_{A} \overrightarrow{A C}$. 
Proof : Let $A \prec C \prec B$. This means by Definition 9 that $\frac{\langle\overrightarrow{C A}, \overrightarrow{C B}\rangle_{A}}{\|\overrightarrow{C A}\|_{A}|| \overrightarrow{C B} \|_{A}}=-1$. Then, it follows by Theorem 1.10 Part 3 that there exists a unique negative real number

$$
t_{B}=1-\frac{\|\overrightarrow{A B}\|_{A}}{\|\overrightarrow{C B}\|_{A}}
$$

and

$$
\left(t_{B}\right) \overrightarrow{C B}={ }_{A} \overrightarrow{C A}, t_{B}<0 .
$$

Also, by Part 2 from Theorem 1.10, we have

$$
\|\overrightarrow{C A}\|_{A}=\|\overrightarrow{A B}\|_{A}-\|\overrightarrow{B C}\|_{A}=\left(1-\frac{\|\overrightarrow{B C}\|_{A}}{\|\overrightarrow{A B}\|_{A}}\right)\|\overrightarrow{A B}\|_{A}
$$

If we put $t_{0}=1-\frac{\|\overrightarrow{B C}\|_{A}}{\|\left.\overrightarrow{A B}\right|_{A}}$, then we have by Part 2 from Theorem 1.10 that $1>\frac{\|\overrightarrow{B C}\|_{A}}{\|\overrightarrow{A B}\|_{A}}$. So, we conclude that

$$
0<t_{0}<1
$$

Now, we show that

$$
\overrightarrow{A C} \mathfrak{R}\left(t_{0}\right) \overrightarrow{A B}
$$

Notice that since $t_{0}>0$ and by Equation (3.6) we have

$$
\left\|\left(t_{0}\right) \overrightarrow{A B}\right\|_{A}=t_{0}\left\|\left.\overrightarrow{A B}\right|_{A}=\left(1-\frac{\|\left.\overrightarrow{B C}\right|_{A}}{\|\overrightarrow{A B}\|_{A}}\right)\right\| \overrightarrow{A B}\left\|_{A}=\right\| \overrightarrow{C A}\left\|_{A}=\right\| \overrightarrow{A C} \|_{A}
$$

Also, by Definition 4 and Equation (3.5) we have

$$
\left(-t_{B}\right) \overrightarrow{C B}={ }_{A} \overrightarrow{A C}
$$

where $-t_{B}>0$. Now, since $t_{0}>0$ and using Equations (1.8), (3.10), and Lemma 1.9 we can write

$$
\frac{\left\langle\overrightarrow{A C},\left(t_{0}\right) \overrightarrow{A B}\right\rangle_{A}}{\|\overrightarrow{A C}\|_{A}\left\|\left(t_{0}\right) \overrightarrow{A B}\right\|_{A}}=\frac{-t_{0} t_{B}\langle\overrightarrow{C B}, \overrightarrow{A B}\rangle_{A}}{t_{0}\left|-t_{B}\right|\|\overrightarrow{C B}\|_{A}\|\overrightarrow{A B}\|_{A}}=\frac{\langle\overrightarrow{C B}, \overrightarrow{A B}\rangle_{A}}{\|\overrightarrow{C B}\|_{A}\|\overrightarrow{A B}\|_{A}}
$$

Applying Definition 5 and Equation (1.5) to the right hand side in the above yields

$$
\frac{\left\langle\overrightarrow{A C},\left(t_{0}\right) \overrightarrow{A B}\right\rangle_{A}}{\|\overrightarrow{A C}\|_{A}\left\|\left(t_{0}\right) \overrightarrow{A B}\right\|_{A}}=\frac{\langle\overrightarrow{C B}, \overrightarrow{A C}\rangle_{A}+\langle\overrightarrow{C B}, \overrightarrow{C B}\rangle_{A}}{\|\overrightarrow{C B}\|_{A}\|\overrightarrow{A B}\|_{A}}
$$


This can be rewritten, using Equations (1.8), (3.10) and Definition 6, as follows

$$
\begin{gathered}
\frac{\left\langle\overrightarrow{A C},\left(t_{0}\right) \overrightarrow{A B}\right\rangle_{A}}{\|\overrightarrow{A C}\|_{A}\left\|\left(t_{0}\right) \overrightarrow{A B}\right\|_{A}}=\frac{\left\langle\overrightarrow{C B},\left(-t_{B}\right) \overrightarrow{C B}\right\rangle_{A}+\langle\overrightarrow{C B}, \overrightarrow{C B}\rangle_{A}}{\|\overrightarrow{C B}\|\left\|_{A}\right\| \overrightarrow{A B} \|_{A}} \\
=\frac{\left(-t_{B}\right)\|\overrightarrow{C B}\|_{A}^{2}+\|\overrightarrow{C B}\|_{A}^{2}}{\|\overrightarrow{C B}\|_{A}\|\overrightarrow{A B}\|_{A}} .
\end{gathered}
$$

By rearranging the preceding equation and by 3.4 we have

$$
\begin{aligned}
\frac{\left\langle\overrightarrow{A C},\left(t_{0}\right) \overrightarrow{A B}\right\rangle_{A}}{\|\overrightarrow{A C}\|_{A}\left\|\left(t_{0}\right) \overrightarrow{A B}\right\|_{A}} & =\left(1-t_{B}\right) \frac{\|\overrightarrow{C B}\|_{A}}{\|\overrightarrow{A B}\|_{A}} \\
& =\left(1-\left(1-\frac{\|\overrightarrow{A B}\|_{A}}{\|\overrightarrow{C B}\|_{A}}\right)\right) \frac{\|\overrightarrow{C B}\|_{A}}{\|\overrightarrow{A B}\|_{A}}=1 .
\end{aligned}
$$

Combining Equations (3.11) and (3.9) with Definition 10 shows that Equation (3.8) holds, that is, $\overrightarrow{A C} \Re\left(t_{0}\right) \overrightarrow{A B}$. Using Definition 7, let

$$
\left(t_{0}\right) \overrightarrow{A B}={ }_{A} \overrightarrow{A D}
$$

for some point $D$. Then, it follows by Equation (3.8) that $\overrightarrow{A C} \Re \overrightarrow{A D}$. Hence, Proposition 1.5 implies that $C={ }_{P} D$. Therefore, Equation (3.12) becomes $\left(t_{0}\right) \overrightarrow{A B}={ }_{A} \overrightarrow{A D}$, where $0<t<1$ (by Equation (3.7)) which ends the proof of this Theorem.

Next, let $A, B$, and $C$ be three distinct points on a line. Consider the three pairs of arrows each of which emanate from the same point, that is,

$$
\{\overrightarrow{A B}, \overrightarrow{A C}\},\{\overrightarrow{C A}, \overrightarrow{C B}\} \text {, and }\{\overrightarrow{B A}, \overrightarrow{B C}\}
$$

Then, we show that one and only one of the pre-inner products

$$
\frac{\langle\overrightarrow{A B}, \overrightarrow{A C}\rangle_{A}}{\|\overrightarrow{A B}\|_{A}\|\overrightarrow{A C}\|_{A}}, \frac{\langle\overrightarrow{C A}, \overrightarrow{C B}\rangle_{A}}{\|\overrightarrow{C A}\|_{A}\|\overrightarrow{C B}\|_{A}} \text {, and } \frac{\langle\overrightarrow{B A}, \overrightarrow{B C}\rangle_{A}}{\|\overrightarrow{B A}\|_{A}\|\overrightarrow{B C}\|_{A}}
$$

is equal to $(-1)$. Notice that, in an arrow space, by means of Definition 9 this result affirms that one and only one of the three points lie between the other two. This is the third betweenness axiom in Hilbert's Axioms of Euclidean Plane Geometry. This result is a consequence of Proposition 1.8.

Theorem 3.3. (The Axiom B3 of Betweenness) Given three distinct points on a line, then one and only one of them is between the other two. 
Proof : Let $A, B$, and $C$ be three distinct points on a line $l$. Then, by the uniqueness of line from Theorem 1.11 and by means of Definition 8 , we can write $\overrightarrow{A B}={ }_{A}(t) \overrightarrow{A C}$ for some real number $t$. Moreover, since $A \neq{ }_{P} B$, we have by Lemma 1.1 that $t \neq 0$. Now, it follows by Proposition 1.8 that

$$
\frac{\langle\overrightarrow{A B}, \overrightarrow{A C}\rangle_{A}}{\|\overrightarrow{A B}\|_{A}\|\overrightarrow{A C}\|_{A}}= \pm 1
$$

The above equation proposes two possible cases.

Case 1. We start with case where

$$
\frac{\langle\overrightarrow{A B}, \overrightarrow{A C}\rangle_{A}}{\|\overrightarrow{A B}\|_{A}\|\overrightarrow{A C}\|_{A}}=-1
$$

By Definition 9, the preceding equation implies that $B \prec A \prec C$, that is, at least one of the three points lies between the other two. Then, it follows by Part 2 from Theorem 1.10 that

$$
\|\overrightarrow{B C}\|_{A}=\|\overrightarrow{B A}\|_{A}+\|\overrightarrow{A C}\|_{A}
$$

We show that it is the only point that lies between the other two. Since we have by Proposition 1.8 that the pre-inner product of any two unit arrows, that emanate from the same point and lie on a line, must be either 1 or -1 , we need only to show that

$$
\frac{\langle\overrightarrow{C A}, \overrightarrow{C B}\rangle_{A}}{\|\overrightarrow{C A}\|_{A}\|\overrightarrow{C B}\|_{A}}=1
$$

and

$$
\frac{\langle\overrightarrow{B A}, \overrightarrow{B C}\rangle_{A}}{\|\overrightarrow{B A}\|_{A}\|\overrightarrow{B C}\|_{A}}=1
$$

The proof of the two preceding equations, namely (3.16) and (3.17), is the same. So, we only prove Equation (3.16). Now, we can use Definition 5 and Equation (1.5) to write the left hand side of Equation (3.16) as follows

$$
\frac{\langle\overrightarrow{C A}, \overrightarrow{C B}\rangle_{A}}{\|\overrightarrow{C A}\|_{A}\|\overrightarrow{C B}\|_{A}}=\frac{\left\langle\overrightarrow{C A}, \overrightarrow{C A}+_{A} \overrightarrow{A B}\right\rangle_{A}}{\|\overrightarrow{C A}\|_{A}\|\overrightarrow{C B}\|_{A}}=\frac{\langle\overrightarrow{C A}, \overrightarrow{C A}\rangle_{A}+\langle\overrightarrow{C A}, \overrightarrow{A B}\rangle_{A}}{\|\overrightarrow{C A}\|_{A}\|\overrightarrow{C B}\|_{A}}
$$

This is equivalent, by Definition 6 and Equation (1.6), to

$$
\frac{\langle\overrightarrow{C A}, \overrightarrow{C B}\rangle_{A}}{\|\overrightarrow{C A}\|_{A}\|\overrightarrow{C B}\|_{A}}=\frac{\|\overrightarrow{C A}\|_{A}^{2}-\langle\overrightarrow{A C}, \overrightarrow{A B}\rangle_{A}}{\|\overrightarrow{C A}\|_{A}\|\overrightarrow{C B}\|_{A}}
$$


If we split the right hand side into two fractions and multiply the second fraction by $\frac{\|\overrightarrow{A B}\|_{A}}{\|\overrightarrow{A B}\|_{A}}$, we get

$$
\frac{\langle\overrightarrow{C A}, \overrightarrow{C B}\rangle_{A}}{\|\overrightarrow{C A}\|_{A}\|\overrightarrow{C B}\|_{A}}=\frac{\|\overrightarrow{C A}\|_{A}^{2}}{\|\overrightarrow{C A}\|_{A}\|\overrightarrow{C B}\|_{A}}+\frac{-\langle\overrightarrow{A C}, \overrightarrow{A B}\rangle_{A}\|\overrightarrow{A B}\|_{A}}{\|\overrightarrow{C A}\|_{A}\|\overrightarrow{C B}\|_{A}\|\overrightarrow{A B}\|_{A}}
$$

This simplifies by Equation (3.14) to

$$
\frac{\langle\overrightarrow{C A}, \overrightarrow{C B}\rangle_{A}}{\|\overrightarrow{C A}\|_{A}\|\overrightarrow{C B}\|_{A}}=\frac{\|\overrightarrow{C A}\|_{A}}{\|\overrightarrow{C B}\|_{A}}+\frac{\|\overrightarrow{A B}\|_{A}}{\|\overrightarrow{C B}\|_{A}}=\frac{\|\overrightarrow{C A}\|_{A}+\|\overrightarrow{A B}\|_{A}}{\|\overrightarrow{C B}\|_{A}}
$$

Now, by Equation (3.15), the above equation becomes

$$
\frac{\langle\overrightarrow{C A}, \overrightarrow{C B}\rangle_{A}}{\|\overrightarrow{C A}\|_{A}\|\overrightarrow{C B}\|_{A}}=\frac{\|\overrightarrow{C B}\|_{A}}{\|\overrightarrow{C B}\|_{A}}=1
$$

This ends the proof of the first case; and now we deal with the second one.

Case 2. We consider the case where

$$
\frac{\langle\overrightarrow{A B}, \overrightarrow{A C}\rangle_{A}}{\|\overrightarrow{A B}\|_{A}\|\overrightarrow{A C}\|_{A}}=1
$$

Notice that we have, by means of Proposition 1.8, that

$$
\frac{\langle\overrightarrow{B A}, \overrightarrow{B C}\rangle_{A}}{\|\overrightarrow{B A}\|_{A}\|\overrightarrow{B C}\|_{A}}= \pm 1
$$

Now, if

$$
\frac{\langle\overrightarrow{B A}, \overrightarrow{B C}\rangle_{A}}{\|\overrightarrow{B A}\|_{A}\|\overrightarrow{B C}\|_{A}}=-1
$$

then a similar argument to the one that we provided in the first case of this proof would lead to the conclusion that we must have

$$
\frac{\langle\overrightarrow{C A}, \overrightarrow{C B}\rangle_{A}}{\|\overrightarrow{C A}\|_{A}\|\overrightarrow{C B}\|_{A}}=1
$$


Then, by means of Equation (3.20) and Definition 9, Equations (3.18), (3.20), and (3.21) mean that we only have $A \prec B \prec C$, as desired. If not, that is, if

$$
\frac{\langle\overrightarrow{B A}, \overrightarrow{B C}\rangle_{A}}{\|\overrightarrow{B A}\|_{A}\|\overrightarrow{B C}\|_{A}}=1
$$

then to finish the proof of this theorem and since both right hand sides of Equations (3.18) and (3.22) is 1 , we need to show that

$$
\frac{\langle\overrightarrow{C A}, \overrightarrow{C B}\rangle_{A}}{\|\overrightarrow{C A}\|_{A}\|\overrightarrow{C B}\|_{A}}=-1 \text {. }
$$

Indeed, applying Part 2 in Theorem 1.9 to Equation 3.22 yields

$$
\|\overrightarrow{C B}\|_{A}=\|\overrightarrow{A B}\|_{A}-\|\overrightarrow{C A}\|_{A}
$$

Also, we can use Definition 5 and Equation (1.5) to write the left hand side of Equation (3.23) as follows

$$
\frac{\langle\overrightarrow{C A}, \overrightarrow{C B}\rangle_{A}}{\|\overrightarrow{C A}\|_{A}\|\overrightarrow{C B}\|_{A}}=\frac{\left\langle\overrightarrow{C A}, \overrightarrow{C A}+{ }_{A} \overrightarrow{A B}\right\rangle_{A}}{\|\overrightarrow{C A}\|_{A}\|\overrightarrow{C B}\|_{A}}=\frac{\langle\overrightarrow{C A}, \overrightarrow{C A}\rangle_{A}+\langle\overrightarrow{C A}, \overrightarrow{A B}\rangle_{A}}{\|\overrightarrow{C A}\|_{A}\|\overrightarrow{C B}\|_{A}}
$$

This is equivalent, by Definition 6 and Equation (1.6), to

$$
\frac{\langle\overrightarrow{C A}, \overrightarrow{C B}\rangle_{A}}{\|\overrightarrow{C A}\|_{A}\|\overrightarrow{C B}\|_{A}}=\frac{\|\overrightarrow{C A}\|_{A}^{2}-\langle\overrightarrow{A C}, \overrightarrow{A B}\rangle_{A}}{\|\overrightarrow{C A}\|_{A}\|\overrightarrow{C B}\|_{A}}
$$

If we split the right hand side of the preceding equation into two fractions and multiply the second fraction by $\frac{\|\overrightarrow{A B}\|_{A}}{\|\overrightarrow{A B}\|_{A}}$, we get

$$
\frac{\langle\overrightarrow{C A}, \overrightarrow{C B}\rangle_{A}}{\|\overrightarrow{C A}\|_{A}\|\overrightarrow{C B}\|_{A}}=\frac{\|\overrightarrow{C A}\|_{A}^{2}}{\|\overrightarrow{C A}\|_{A}\|\overrightarrow{C B}\|_{A}}-\frac{\langle\overrightarrow{A C}, \overrightarrow{A B}\rangle_{A}\|\overrightarrow{A B}\|_{A}}{\|\overrightarrow{C A}\|_{A}\|\overrightarrow{C B}\|_{A}\|\overrightarrow{A B}\|_{A}}
$$

This simplifies by Equation (3.18) to

$$
\frac{\langle\overrightarrow{C A}, \overrightarrow{C B}\rangle_{A}}{\|\overrightarrow{C A}\|_{A}\|\overrightarrow{C B}\|_{A}}=\frac{\|\overrightarrow{C A}\|_{A}}{\|\overrightarrow{C B}\|_{A}}-\frac{\|\overrightarrow{A B}\|_{A}}{\|\overrightarrow{C B}\|_{A}}=\frac{\|\overrightarrow{C A}\|_{A}-\|\overrightarrow{A B}\|_{A}}{\|\overrightarrow{C B}\|_{A}} .
$$

Now, by Equation (3.24), the above equation becomes

$$
\frac{\langle\overrightarrow{C A}, \overrightarrow{C B}\rangle_{A}}{\|\overrightarrow{C A}\|_{A}\|\overrightarrow{C B}\|_{A}}=\frac{-\|\overrightarrow{C B}\|_{A}}{\|\overrightarrow{C B}\|_{A}}=-1
$$


This ends the proof of this theorem.

Next, we provide a criterion by which one can tell if a point is between two other points on a line.

Proposition 3.1. Let $B, C$ and $D$ be three distinct points on a line. If

$$
\|\overrightarrow{B C}\|_{A}=\|\overrightarrow{B D}\|_{A}+\|\overrightarrow{D C}\|_{A}
$$

then $B \prec D \prec C$.

Proof : First notice that since the points $B, C$ and $D$ are distinct, then it follows by Lemma 1.1 that

$$
\|\overrightarrow{B C}\|_{A} \neq 0,\|\overrightarrow{B D}\|_{A} \neq 0,\|\overrightarrow{D C}\|_{A} \neq 0
$$

Also, since $B, C$ and $D$ are distinct, Proposition 1.8 implies that

$$
\frac{\langle\overrightarrow{B D}, \overrightarrow{B C}\rangle_{A}}{\|\overrightarrow{B D}\|_{A}\|\overrightarrow{B C}\|_{A}}= \pm 1, \frac{\langle\overrightarrow{C D}, \overrightarrow{C B}\rangle_{A}}{\|\overrightarrow{C D}\|_{A}\|\overrightarrow{C B}\|_{A}}= \pm 1, \text { and } \frac{\langle\overrightarrow{D C}, \overrightarrow{D B}\rangle_{A}}{\|\overrightarrow{D C}\|_{A}\|\overrightarrow{D B}\|_{A}}= \pm 1
$$

If

$$
\frac{\langle\overrightarrow{B D}, \overrightarrow{B C}\rangle_{A}}{\|\overrightarrow{B D}\|_{A}\|\overrightarrow{B C}\|_{A}}=-1, \text { or } \frac{\langle\overrightarrow{C D}, \overrightarrow{C B}\rangle_{A}}{\|\overrightarrow{C D}\|_{A}\|\overrightarrow{C B}\|_{A}}=-1
$$

then we have by Part 2 in Theorem 1.10 that

$$
\left\|\left.\overrightarrow{B C}\right|_{A}=\right\| \overrightarrow{C D}\left\|_{A}-\right\| \overrightarrow{B D} \|_{A} \text {, or }\|\overrightarrow{B C}\|_{A}=\|\overrightarrow{B D}\|_{A}-\|\overrightarrow{D C}\|_{A}
$$

But the two equations in the above are impossible under the hypothesis of this proposition. Indeed, adding up the first and the second part of Equation (3.29) separately to Equation (3.25) yields

$$
\|\overrightarrow{B C}\|_{A}=\|\overrightarrow{C D}\|_{A}, \text { or }\|\overrightarrow{B C}\|_{A}=\|\overrightarrow{B D}\|_{A}
$$

Combining each of the preceding equations, separately, with Equation (3.25) gives

$$
\|\overrightarrow{B D}\|_{A}=0, \text { or }\|\overrightarrow{C D}\|_{A}=0
$$

each of the above equations contradicts Equation (3.26). Thus, the two equations in 3.28 are impossible. So, we must have

$$
\frac{\langle\overrightarrow{B D}, \overrightarrow{B C}\rangle_{A}}{\|\overrightarrow{B D}\|_{A}\|\overrightarrow{B C}\|_{A}}=1, \text { and } \frac{\langle\overrightarrow{C D}, \overrightarrow{C B}\rangle_{A}}{\|\overrightarrow{C D}\|_{A}\|\overrightarrow{C B}\|_{A}}=1
$$


Now, by means of Definition 9, equations (3.27), (3.30), and Theorem 3.3 we must have

$$
\frac{\langle\overrightarrow{D C}, \overrightarrow{D B}\rangle_{A}}{\|\overrightarrow{D C}\|_{A}\|\overrightarrow{D B}\|_{A}}=-1
$$

Combining the preceding equation and Definition 9 shows that $B \prec D \prec C$, as desired.

The following theorem embodies the second axiom of betweenness in Hilbert's Axioms of Euclidean Plane Geometry. This axiom says that for any two distinct points $A, B$ there exists a point $C$ such that $A \prec B \prec C$. In fact, in an arrow space, as a reflection of Axiom 3, there are infinitely many points $C$. We reformulate and prove this axiom using the language of arrow spaces as follows.

Theorem 3.4. (The Axiom B2 of Betweenness) Let $A, B$ be two distinct points. Then, for any $t>1$ and any point $C$ such that $\overrightarrow{A C}={ }_{A}(t) \overrightarrow{A B}$ we have

$$
\frac{\langle\overrightarrow{B A}, \overrightarrow{B C}\rangle_{A}}{\|\overrightarrow{B A}\|_{A}\|\overrightarrow{B C}\|_{A}}=-1
$$

Proof : Let $A, B$ be two distinct points and $l_{A B}$ be the line that contains them. Then, it follows by Definition 8 that, for any $t>1$ and any point $C$ with

$$
\overrightarrow{A C}={ }_{A}(t) \overrightarrow{A B}
$$

such point $C$ lies on $l_{A B}$. Moreover, by Definition 9, Equations (1.8) and (1.9) and since $t>1$ we have

$$
\frac{\langle\overrightarrow{A C}, \overrightarrow{A B}\rangle_{A}}{\|\overrightarrow{A C}\|_{A}\|\overrightarrow{A B}\|_{A}}=\frac{\langle(t) \overrightarrow{A B}, \overrightarrow{A B}\rangle_{A}}{\|(t) \overrightarrow{A B}\|_{A}\|\overrightarrow{A B}\|_{A}}=\frac{t\langle\overrightarrow{A B}, \overrightarrow{A B}\rangle_{A}}{t\|\overrightarrow{A B}\|_{A}\|\overrightarrow{A B}\|_{A}}=1 .
$$

We also have by Equations (3.33) and (1.9) that

$$
\|\overrightarrow{A C}\|_{A}=\|(t) \overrightarrow{A B}\|_{A}=t\|\overrightarrow{A B}\|_{A}
$$

Dividing both sides of the above by $t$ and applying Equation (1.9) yields

$$
\left(\frac{1}{t}\right)\|\overrightarrow{A C}\|_{A}=\left\|\left(\frac{1}{t}\right) \overrightarrow{A C}\right\|_{A}=\|\overrightarrow{A B}\|_{A} .
$$

Also, by Equations (1.8), (1.9), and (3.34) we have

$$
\frac{\left\langle\left(\frac{1}{t}\right) \overrightarrow{A C}, \overrightarrow{A B}\right\rangle_{A}}{\left\|\left(\frac{1}{t}\right) \overrightarrow{A C}\right\|_{A}\|\overrightarrow{A B}\|_{A}}=\frac{\left(\frac{1}{t}\right)\langle\overrightarrow{A C}, \overrightarrow{A B}\rangle_{A}}{\left(\frac{1}{t}\right)\|\overrightarrow{A C}\|_{A}\|\overrightarrow{A B}\|_{A}}=\frac{\langle\overrightarrow{A C}, \overrightarrow{A B}\rangle_{A}}{\|\overrightarrow{A C}\|_{A}\|\overrightarrow{A B}\|_{A}}=1
$$


It follows by Equations (3.35), (3.36), and Proposition 1.5 that

$$
\overrightarrow{A B}={ }_{A}\left(\frac{1}{t}\right) \overrightarrow{A C}
$$

Since $t>1$, it follows that $0<\frac{1}{t}<1$. Then, according to Theorem 3.1, the preceding equation means that $A \prec B \prec C$, which by Definition 9 means that

$$
\frac{\langle\overrightarrow{B A}, \overrightarrow{B C}\rangle_{A}}{\|\overrightarrow{B A}\|_{A}\|\overrightarrow{B C}\|_{A}}=-1
$$

as desired.

We end this Section by giving another criterion, this time involving four distinct points, for a point being between two other points on a line. This fact will contribute to the proof of The Plane Separation Theorem, see Theorem 3.10 in Section 3.3.

Proposition 3.2. Let $A, B, C$ and $D$ be three distinct points on a line. If

$$
A \prec B \prec C, A \prec B \prec D \text {, and } A \prec D \prec C \text {, }
$$

then we must have $B \prec D \prec C$.

Proof : Notice that, by Definition 9, the three betweenness relations in 3.39 mean that

$$
\frac{\langle\overrightarrow{B A}, \overrightarrow{B C}\rangle_{A}}{\|\overrightarrow{A B}\|_{A}\|\overrightarrow{B C}\|_{A}}=\frac{\langle\overrightarrow{B A}, \overrightarrow{B D}\rangle_{A}}{\|\overrightarrow{B A}\|_{A}\|\overrightarrow{B D}\|_{A}}=\frac{\langle\overrightarrow{D A}, \overrightarrow{D C}\rangle_{A}}{\|\overrightarrow{D A}\|_{A}\|\overrightarrow{D C}\|_{A}}=-1
$$

The above equations imply by Part 2 from Theorem 1.10 that

$$
\begin{aligned}
& \|\overrightarrow{B C}\|_{A}=\|\overrightarrow{A C}\|_{A}-\|\overrightarrow{A B}\|_{A} \\
& \|\overrightarrow{B D}\|_{A}=\|\overrightarrow{A D}\|_{A}-\|\overrightarrow{A B}\|_{A} \\
& \|\overrightarrow{A D}\|_{A}=\|\overrightarrow{A C}\|_{A}-\|\overrightarrow{D C}\|_{A}
\end{aligned}
$$

Subtracting Equation (3.41) from Equation (3.42) gives

$$
\|\overrightarrow{B D}\|_{A}-\|\overrightarrow{B C}\|_{A}=\|\overrightarrow{A D}\|_{A}-\|\overrightarrow{A C}\|_{A}
$$

Combining Equations (3.43) and (3.44) and rearranging yields

$$
\begin{aligned}
\|\overrightarrow{B D}\|_{A}-\|\overrightarrow{B C}\|_{A} & =\|\overrightarrow{A D}\|_{A}-\|\overrightarrow{A C}\|_{A} \\
& =\|\overrightarrow{A C}\|_{A}-\|\overrightarrow{D C}\|_{A}-\|\overrightarrow{A C}\|_{A} \\
& =-\|\overrightarrow{D C}\|_{A},
\end{aligned}
$$


which is equivalent to

$$
\|\overrightarrow{B C}\|_{A}=\|\overrightarrow{B D}\|_{A}-\|\overrightarrow{D C}\|_{A}
$$

Now, since $B, C$, and $D$ are distinct points, it follows by the preceding equation and Proposition 3.1 that $B \prec D \prec C$, as desired.

\subsection{Angles and trigonometry in arrow spaces}

In [20] page 76, Hartshorne proves the Line Separation Theorem, while relying on the Plane Separation Theorem (3.10). We, on the other hand, prove the Line Separation Theorem (see Theorem 3.5) in arrow spaces by strictly using Axiom 3 and Theorem 1.1. While we still prove Theorem 3.10, our proof of the Line Separation Theorem is independent from Theorem 3.10. Regardless, the Plane Separation Theorem follows as an application of Theorem 3.1 (Pasch's Axiom) in our setting and in [20]. One of the main goals of this section is to introduce the Cosine function, a key tool needed to prove Theorem 3.1. In order to introduce the Cosine function, we first define a ray and then an angle between two rays. We begin this section with the Line Separation Theorem.

Theorem 3.5. (Line Separation Theorem in Arrow Spaces) Let $A$ and $B$ be two distinct points and $l_{A B}$ be the line that contains $A, B$. Then, the set of all points of $l \backslash\{A\}$ can be divided into two disjoint nonempty subsets $P_{+}, P_{-}$, the two sides of $A$ on $l$, where

$$
\begin{gathered}
P_{+}=\left\{P \in l \mid \overrightarrow{A P}={ }_{A}(t) \overrightarrow{A B}, t>0\right\}, \text { and } \\
P_{-}=\left\{P \in l \mid \overrightarrow{A P}={ }_{A}(t) \overrightarrow{A B}, t<0\right\} .
\end{gathered}
$$

Proof : First notice that by Axiom 3 each of $P_{+}$and $P_{-}$is nonempty. Next, we show that $P_{+}$and $P_{-}$do not have points in common. So, assume, by way of contradiction, that there exists a point $C \in\left(P_{+} \cap P_{-}\right)$. This means, by 3.46 and 3.47 , that there exist $t>0$ and $s<0$ such that

$$
\overrightarrow{A C}={ }_{A}(t) \overrightarrow{A B}, \overrightarrow{A C}=_{A}(s) \overrightarrow{A B}
$$

The equations in 3.48 imply that $(t) \overrightarrow{A B}={ }_{A}(s) \overrightarrow{A B}$ for some $s, t \neq 0$. Then, it follows according to Theorem 1.1 that $s=t$. This is a contradiction as $t>0$ and $s<0$. This means that the intersection of $P_{+}$and $P_{-}$is an empty set.

We learned from Proposition 1.8 that for any three distinct points $A, B$, and $C$ on a line $l$ we always have

$$
\left\langle\frac{\overrightarrow{A B}}{\|\overrightarrow{A B}\|_{A}}, \frac{\overrightarrow{A C}}{\|\overrightarrow{A C}\|_{A}}\right\rangle_{A}= \pm 1
$$


If $l$ is divided by $A$, then the following proposition rigorously tells us when this pre inner product is 1 and when it is -1 .

Proposition 3.3. Let $l_{A P}$ be a line that is divided by a point A. Given any two points $B, C$ other than $A$. Then,

1. if $B, C$ are in the same side of $A$, we have

$$
\left\langle\frac{\overrightarrow{A B}}{\|\overrightarrow{A B}\|_{A}}, \frac{\overrightarrow{A C}}{\|\overrightarrow{A C}\|_{A}}\right\rangle_{A}=1
$$

2. if $B, C$ are in the opposite side of $A$, we have

$$
\left\langle\frac{\overrightarrow{A B}}{\|\overrightarrow{A B}\|_{A}}, \frac{\overrightarrow{A C}}{\|\overrightarrow{A C}\|_{A}}\right\rangle_{A}=-1
$$

\section{Proof :}

1. If $B, C$ are in the same side of $A$, then it follows by Theorem 3.5 that there exist $s, t>0$ such that $\overrightarrow{A B}={ }_{A}(s) \overrightarrow{A P}$ and $\overrightarrow{A C}={ }_{A}(t) \overrightarrow{A P}$. Hence, since $s, t>0$, using Equations 1.9,1.8, and Definition 6, we have

$$
\left\langle\frac{\overrightarrow{A B}}{\|\overrightarrow{A B}\|_{A}}, \frac{\overrightarrow{A C}}{\|\overrightarrow{A C}\|_{A}}\right\rangle_{A}=\left\langle\frac{(s) \overrightarrow{A P}}{\|(s) \overrightarrow{A P}\|_{A}}, \frac{(t) \overrightarrow{A P}}{\|(t) \overrightarrow{A P}\|_{A}}\right\rangle_{A}=1
$$

2. If $B, C$ are in the opposite side of $A$, then it follows by Theorem 3.5 that there exist $s<0, t>0$ such that $\overrightarrow{A B}={ }_{A}(s) \overrightarrow{A P}$ and $\overrightarrow{A C}={ }_{A}(t) \overrightarrow{A P}$. Hence, since $s<0, t>0$, using Equations 1.9,1.8, and Definition 6, we have

$$
\left\langle\frac{\overrightarrow{A B}}{\|\overrightarrow{A B}\|_{A}}, \frac{\overrightarrow{A C}}{\|\overrightarrow{A C}\|_{A}}\right\rangle_{A}=\left\langle\frac{(s) \overrightarrow{A P}}{\|(s) \overrightarrow{A P}\|_{A}}, \frac{(t) \overrightarrow{A P}}{\|(t) \overrightarrow{A P}\|_{A}}\right\rangle_{A}=-1
$$

We now define a ray which will be used in the definition of an angle.

Definition 16. Let $A, B$ be two distinct points and $l$ be the line that contains $A, B$. A ray that emanates from $A$ and contains $B$, denoted by $\overrightarrow{A B}_{\infty}$, is the set

$$
\overrightarrow{A B}_{\infty}=\left\{P \in l \mid \overrightarrow{A P}=_{A}(t) \overrightarrow{A B}, t \geq 0\right\}
$$


Notice that, in the above definition, if $t=0$, then we would have by Definition 7 that

$$
\overrightarrow{A P}={ }_{A}(0) \overrightarrow{A B}={ }_{A} \overrightarrow{A A}
$$

This means by Definition 3 that $P={ }_{P} A$. Hence, we conclude from this and Definition 16 that $A \in \overrightarrow{A B}_{\infty}$.

Definition 17. (An Angle in arrow spaces) Let $A, B$, and $C$ be three points such that $A \neq B, A \neq C$. Consider the arrows $\overrightarrow{A B}, \overrightarrow{A C}$. An angle between the two rays $\overrightarrow{A B}_{\infty}$ and $\overrightarrow{A B}_{\infty}$ is the union of the two rays $\overrightarrow{A B}_{\infty}$ and $\overrightarrow{A B}_{\infty}$ and is denoted by $\angle C A B$ or $\angle B A C$.

Definition 18. Given an angle $\angle C A B$. We define the cosine of the angle $\angle C A B$, denoted by $\cos \angle C A B$, to be given by the equation

$$
\cos \angle C A B=\frac{\langle\overrightarrow{A C}, \overrightarrow{A B}\rangle_{A}}{\|\overrightarrow{A C}\|_{A}\|\overrightarrow{A B}\|_{A}} .
$$

Remark 3.1. In Definition 17, we mentioned that the two notations $\angle B A C, \angle C A B$ are to be used to refer to one angle. Now, we show that Definition 18 affirms that the cosine of each of $\angle B A C$ and $\angle C A B$ has the same value. This follows from Equation (1.2) which says that $\langle\overrightarrow{A B}, \overrightarrow{A C}\rangle_{A}=\langle\overrightarrow{A C}, \overrightarrow{A B}\rangle_{A}$. This means that without Equation (1.2) we wouldn't be able to use the two notations, namely $\angle B A C$ and $\angle C A B$, for one angle as we could end up with two different values for the cosine, see Section 4.1 for more details.

Proposition 3.4. Given an angle $\angle C A B$. Then, we have

$$
\cos \angle C A B=\cos \angle B A C .
$$

Proof : By Equation (1.2) and Definition 18 we have

$$
\cos \angle C A B=\frac{\langle\overrightarrow{A C}, \overrightarrow{A B}\rangle_{A}}{\left\|\left.\overrightarrow{A C}\right|_{A}\right\| \overrightarrow{A B} \|_{A}}=\frac{\langle\overrightarrow{A B}, \overrightarrow{A C}\rangle_{A}}{\left\|\left.\overrightarrow{A B}\right|_{A}\right\| \overrightarrow{A C} \|_{A}}=\cos \angle B A C .
$$

Next, we show that Definition 18, of the cosine of an angle $\angle C A B$ between two rays $\overrightarrow{A B}_{\infty}$ and $\overrightarrow{A C}_{\infty}$, does not depend on the choice of $B$ and $C$.

Proposition 3.5. Let $A, B$, and $C$ be three distinct points. Let $\overrightarrow{A B}_{\infty}$ and $\overrightarrow{A C}_{\infty}$ be two rays that emanate from $A$. Then, for any point $D \in \overrightarrow{A B}_{\infty}$ and any point $E \in \overrightarrow{A C}_{\infty}$, where $D, E$ are different than $A$ we have

$$
\cos \angle E A D=\cos \angle C A B .
$$




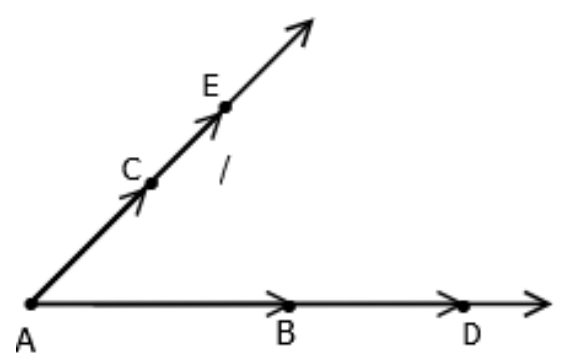

Figure 3.1: The independence of cosine of an angle from the choice of points on the rays, $\cos \angle E A D=\cos \angle C A B$.

Proof : Let $D \in \overrightarrow{A B}_{\infty}$ and $E \in \overrightarrow{A C}_{\infty}$ such that $D \neq_{P} A$ and $E \neq_{P} A$. Then, it follows by Definition 16 that there exist $s, t>0$ such that

$$
\overrightarrow{A D}={ }_{A}(s) \overrightarrow{A B}, \overrightarrow{A E}=_{A}(t) \overrightarrow{A C}
$$

Now, according to the preceding equation and Definition 18, we can write

$$
\cos \angle E A D=\frac{\langle\overrightarrow{A E}, \overrightarrow{A D}\rangle_{A}}{\|\overrightarrow{A E}\|_{A}\|\overrightarrow{A D}\|_{A}}=\frac{\langle(t) \overrightarrow{A C},(s) \overrightarrow{A B}\rangle_{A}}{\|(t) \overrightarrow{A C}\|_{A}\|(s) \overrightarrow{A B}\|_{A}}
$$

Then, by Equations (1.8) and (1.9) and since $s, t>0$, the desired equation holds, that is,

$$
\cos \angle E A D=\frac{(t s)\langle\overrightarrow{A C}, \overrightarrow{A B}\rangle_{A}}{|s||t|\|\overrightarrow{A C}\|_{A}\|\overrightarrow{A B}\|_{A}}=\frac{\langle\overrightarrow{A C}, \overrightarrow{A B}\rangle_{A}}{\|\overrightarrow{A C}\|_{A}\|\overrightarrow{A B}\|_{A}}=\cos \angle C A B .
$$

Remark 3.2. We can conclude from the preceding result (Proposition 3.5) that Definition 18 can be rewritten as $\cos \angle C A B=\langle\overrightarrow{A L}, \overrightarrow{A M}\rangle$, where $\overrightarrow{A L}, \overrightarrow{A M}$ are unit arrows, $L \in \overrightarrow{A B}_{\infty}, M \in \overrightarrow{A C}_{\infty}$

To justify Remark 3.2, we show in the following proposition that such two unit arrows exist.

Proposition 3.6. Let $\overrightarrow{A B}_{\infty}$ be a ray. Then, there exists a unique point $L \in \overrightarrow{A B}_{\infty}$ such that $\overrightarrow{A L}$ is a unit arrow, that is, $\|\overrightarrow{A L}\|_{A}=1$.

Proof : Notice that, by Definition 16, we have for any $D \in \overrightarrow{A B}_{\infty}$ with $D \neq_{p} A$ that

$$
\overrightarrow{A D}={ }_{A}(t) \overrightarrow{A B}, t>0
$$


Therefore, by the preceding equation and Equation (1.9) we have

$$
\|\overrightarrow{A D}\|_{A}=1 \Longleftrightarrow\|(t) \overrightarrow{A B}\|_{A}=1 \Longleftrightarrow t\|\overrightarrow{A B}\|_{A}=1 \Longleftrightarrow t=\frac{1}{\|\overrightarrow{A B}\|_{A}}
$$

Thus, since $A, B$ are fixed it follows that $0<t=\frac{1}{\|\overrightarrow{A B}\|_{A}}$ is unique; and so is the arrow $\overrightarrow{A L}={ }_{A}\left(\frac{1}{\|\overrightarrow{A B}\|_{A}}\right) \overrightarrow{A B}$, for some point $L \in \overrightarrow{A B}_{\infty}$. Also, we have

$$
\|\overrightarrow{A L}\|_{A}=\left\|\left(\frac{1}{\|\overrightarrow{A B}\|_{A}}\right) \overrightarrow{A B}\right\|_{A}=\left(\frac{1}{\|\overrightarrow{A B}\|_{A}}\right)\|\overrightarrow{A B}\|_{A}=1
$$

This ends the proof of the proposition.

In the following definition we introduce vertical angles.

Definition 19. Let $A, B$ and $C$ be three distinct points that do not lie on a line. Consider the angle $\angle C A B$. Let $D$ and $E$ be two points that lie on the opposite sides of $B$ and $C$ of the lines $l_{A B}, l_{A C}$, respectively. We call the angle $\angle E A D$ the vertical angle of the angle $\angle E A D$, see Figure 3.2.

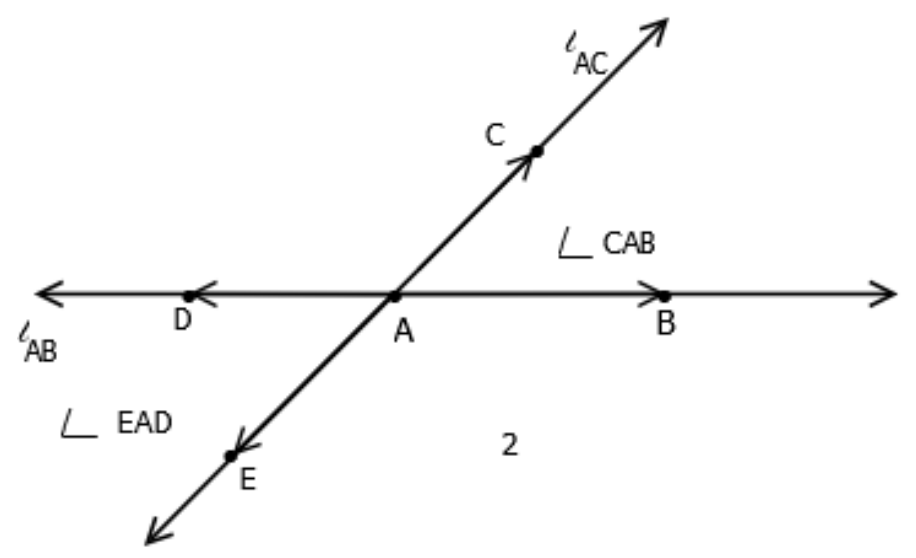

Figure 3.2: Two vertical angles

Now, we show that the cosine of any two vertical angles has the same value.

Theorem 3.6. Let $\angle C A B$ and $\angle E A D$ be two vertical angles, see Figure 3.2. Then, we have

$$
\cos \angle C A B=\cos \angle E A D \text {. }
$$


Proof : Since by Definition 19 we have that $D$ and $E$ lie on the opposite sides of $B$ and $C$ of the lines $l_{A B}, l_{A C}$, respectively, it follows by Definition 16 that there exist $s, t<0$ such that

$$
\overrightarrow{A E}={ }_{A}(s) \overrightarrow{A C}, \overrightarrow{A D}={ }_{A}(t) \overrightarrow{A B}
$$

Then, we have by Definition 18 and Equations (1.8) and (1.9) that

$$
\begin{aligned}
\cos \angle E A D= & \frac{\langle\overrightarrow{A E}, \overrightarrow{A D}\rangle_{A}}{\|\overrightarrow{A E}\|_{A}\|\overrightarrow{A D}\|_{A}}=\frac{\langle(s) \overrightarrow{A C},(t) \overrightarrow{A B}\rangle_{A}}{\|(s) \overrightarrow{A C}\|_{A}\|(t) \overrightarrow{A B}\|_{A}} \\
= & \frac{(s t)\langle\overrightarrow{A C}, \overrightarrow{A B}\rangle_{A}}{|s||t|\|\overrightarrow{A C}\|_{A}\|\overrightarrow{A B}\|_{A}}=\cos \angle C A B
\end{aligned}
$$

where $s t=|s||t|$ at $s, t<0$.

Next, we show that for any angle $\angle C A B$ it is always true that $|\cos \angle C A B| \leq 1$. This can be done after we prove the two following propositions. The first proposition deals with the case where $A, B, C$ lie on the same line.

Proposition 3.7. Given an angle $\angle C A B$, where $A, B$ and $C$ lie on the same line. Then, $\cos \angle C A B=1$ if $B, C$ are in the same side of $A$; and $\cos \angle C A B=-1$ if $B, C$ are in the opposite side of $A$.

Proof : The proof of this proposition follows directly from Proposition 3.3 after considering $A$ as a point of separation of the line $l_{A B}$.

The following proposition considers the case where the three points $A, B$, and $C$ do not lie on the same line.

Proposition 3.8. Let $A, B$, and $C$ be three distinct points that do not lie on the same line. Then, for any point $D \in l_{A B}$ with

$$
\overrightarrow{A D}={ }_{A}(t) \overrightarrow{A B}, t<0
$$

We have $\cos \angle C A B=-\cos \angle C A D$, see Figure 3.3.

Proof : By Definition 18, Equations (1.9), (1.8), and the hypothesis 3.51 we have

$$
\begin{aligned}
\cos \angle C A D & =\frac{\langle\overrightarrow{A C}, \overrightarrow{A D}\rangle_{A}}{\|\overrightarrow{A C}\|_{A}\|\overrightarrow{A D}\|_{A}}=\frac{\langle\overrightarrow{A C},(t) \overrightarrow{A B}\rangle_{A}}{\|\overrightarrow{A C}\|_{A}\|(t) \overrightarrow{A B}\|_{A}} \\
& =\frac{t\langle\overrightarrow{A C}, \overrightarrow{A B}\rangle_{A}}{|t|\|\overrightarrow{A C}\|_{A}\|\overrightarrow{A B}\|_{A}}=\frac{-\langle\overrightarrow{A C}, \overrightarrow{A B}\rangle_{A}}{\|\overrightarrow{A C}\|_{A}\|\overrightarrow{A B}\|_{A}}=-\cos \angle C A B
\end{aligned}
$$

where the negative sign follows from the fact that $t<0$ and so $\frac{t}{|t|}=-1$. 


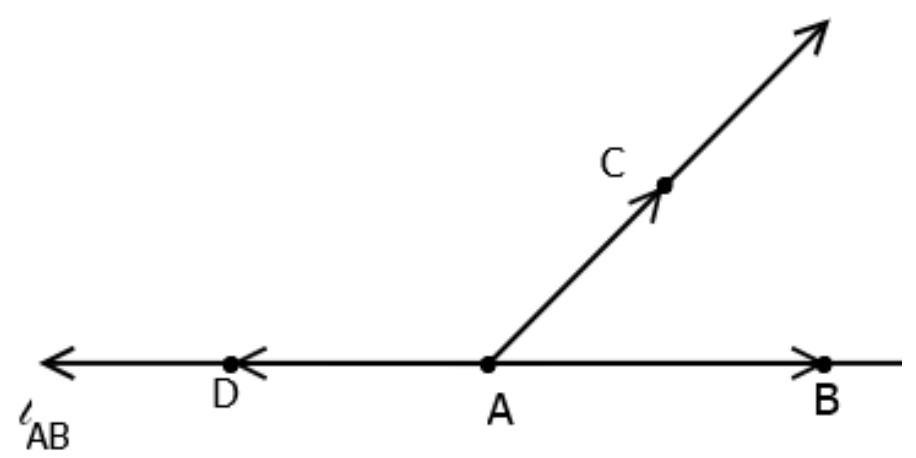

2

Figure 3.3: Two adjacent angles, $\angle C A B, \angle C A D$, for which $\cos \angle C A B=-\cos \angle C A D$

Theorem 3.7. Let $\angle C A B$ be any angle. Then, we have

$$
|\cos \angle C A B| \leq 1
$$

Proof : If $A, B$, and $C$ lie on the same line, then it follows from Proposition 3.7 that $\cos \angle C A B$ is either 1 or -1 . Now, let $A, B$, and $C$ be three distinct points that do not lie on a line. It follows by Proposition 3.6 that there are two points $L \in \overrightarrow{A B}_{\infty}, M \in \overrightarrow{A C}_{\infty}$ such that

$$
\|\overrightarrow{A L}\|_{A}=\|\overrightarrow{A M}\|_{A}=1
$$

Then, by means of Remark 3.2 we have

$$
\cos \angle C A B=\langle\overrightarrow{A L}, \overrightarrow{A M}\rangle .
$$

Now, applications of Equations (1.1), (1.2), (1.5), and Definitions 4, 5, 6 give

$$
\begin{aligned}
0 \leq\|\overrightarrow{L M}\|_{A}^{2} & =\langle\overrightarrow{L M}, \overrightarrow{L M}\rangle=\left\langle\overrightarrow{L A}+_{A} \overrightarrow{A M}, \overrightarrow{L A}+{ }_{A} \overrightarrow{A M}\right\rangle \\
& =\|\overrightarrow{L A}\|_{A}^{2}+2\langle\overrightarrow{L A}, \overrightarrow{A M}\rangle+\|\overrightarrow{A M}\|_{A}^{2}
\end{aligned}
$$

This simplifies, by Equations (3.53) and (1.6), to

$$
0 \leq 2-2\langle\overrightarrow{A L}, \overrightarrow{A M}\rangle
$$

Rearranging the preceding inequality and using Equation (3.54) yields

$$
\cos \angle C A B \leq 1
$$


On the other hand, it follows by Proposition 3.8 that

$$
-\cos \angle C A B=\cos \angle C A N \text {, }
$$

where $N$ is a point on the line $l_{A B}$ in the opposite side of the point $A$ with $(-1) \overrightarrow{A L}={ }_{A}$ $\overrightarrow{A N}$. Now, since Equation (3.55) applies for any angle, including the angle $\angle C A N$, we have from Equation (3.56) that

$$
-\cos \angle C A B=\cos \angle C A N \leq 1
$$

that is,

$$
-1 \leq \cos \angle C A B
$$

Combining Equations (3.55) and (3.57) yields

$$
-1 \leq \cos \angle C A B \leq 1,
$$

which means that $|\cos \angle C A B| \leq 1$, as desired.

Now, we are ready to prove, in arrow spaces, analogous results to Schwars Inequality and The Triangle Inequality, that we have learned in real inner product spaces. We start with a result in an arrow space that mimics Schwars Inequality.

Theorem 3.8. Let $\overrightarrow{A B}, \overrightarrow{B C}$ be two arrows in an arrow space $\mathscr{P}_{A}$. Then, we have

$$
|\langle\overrightarrow{B A}, \overrightarrow{B C}\rangle| \leq\|\overrightarrow{B A}\|_{A}\|\overrightarrow{B C}\|_{A}
$$

where the equality sign holds if and only if exists $t \neq 0$ such that $\overrightarrow{B A}={ }_{A}(t) \overrightarrow{B C}$.

Proof : If $A={ }_{P} B$ (similarly if $B={ }_{P} C$ ), then by Proposition 1.1 and Lemma 1.1 we have

$$
|\langle\overrightarrow{B A}, \overrightarrow{B C}\rangle|=|\langle\overrightarrow{A A}, \overrightarrow{A C}\rangle|=0=\|\overrightarrow{A A}\|_{A}\|\overrightarrow{A C}\|_{A}=\|\overrightarrow{B A}\|_{A}\|\overrightarrow{B C}\|_{A}
$$

If $A \neq \neq_{P} B, B \neq{ }_{A} C$, and $A={ }_{P} C$, then it follows by Definition 6 and Equation (1.6) that

$$
|\langle\overrightarrow{B A}, \overrightarrow{B C}\rangle|=|\langle\overrightarrow{B A}, \overrightarrow{B A}\rangle|=\|\overrightarrow{B A}\|_{A}\|\overrightarrow{A B}\|_{A}=\|\overrightarrow{B A}\|_{A}\|\overrightarrow{B C}\|_{A}
$$

Finally, if $A, B$ and $C$ are distinct points, then it follows by Theorem 3.7 and Definition 18 that

$$
|\cos \angle C A B|=\frac{\left|\langle\overrightarrow{B A}, \overrightarrow{B C}\rangle_{A}\right|}{\|\overrightarrow{B A}\|_{A}\|\overrightarrow{B C}\|_{A}} \leq 1 .
$$


This is equivalent to Equation (3.58), that is

$$
|\langle\overrightarrow{B A}, \overrightarrow{B C}\rangle| \leq\|\overrightarrow{B A}\|_{A}\|\overrightarrow{B C}\|_{A}
$$

For the equality portion of Equation (3.58), we consider only the case where $A, B$ and $C$ are distinct points as we already showed equality in the first two cases in this proof in which points are equal.

First, we show that if $|\langle\overrightarrow{B A}, \overrightarrow{B C}\rangle|=\|\overrightarrow{B A}\|_{A}\|\overrightarrow{B C}\|_{A}$, then exists $t \neq 0$ such that $\overrightarrow{B A}={ }_{A}(t) \overrightarrow{B C}$. Indeed, notice that

$$
|\langle\overrightarrow{B A}, \overrightarrow{B C}\rangle|=\|\overrightarrow{B A}\|_{A}\|\overrightarrow{B C}\|_{A} \Longrightarrow \frac{\langle\overrightarrow{B A}, \overrightarrow{B C}\rangle}{\|\overrightarrow{B A}\|_{A}\|\overrightarrow{B C}\|_{A}}= \pm 1
$$

The preceding equation implies by means of Part 3 from Theorems 1.9 and 1.10 that there exists $t \neq 0$ such that $\overrightarrow{B A}={ }_{A}(t) \overrightarrow{B C}$.

Secondly, if there exists $t \neq 0$ such that $\overrightarrow{B A}={ }_{A}(t) \overrightarrow{B C}$, then by Definition 6 , Equations (1.8) and (1.9) we have

$$
\frac{|\langle\overrightarrow{B A}, \overrightarrow{B C}\rangle|}{\|\overrightarrow{B A}\|_{A}\|\overrightarrow{B C}\|_{A}}=\frac{|(t)\langle\overrightarrow{B C}, \overrightarrow{B C}\rangle|}{|t|\|\overrightarrow{B C}\|_{A}\|\overrightarrow{B C}\|_{A}}=\frac{|t| \|\left.\overrightarrow{B C}\right|_{A} ^{2}}{|t|\|\overrightarrow{B C}\|_{A}\|\overrightarrow{B C}\|_{A}}=1
$$

that is,

$$
|\langle\overrightarrow{B A}, \overrightarrow{B C}\rangle|=\|\overrightarrow{B A}\|_{A}\|\overrightarrow{B C}\|_{A}
$$

Now, we prove the the Triangle Inequality version of arrow spaces.

Theorem 3.9. For any arrows $\overrightarrow{A B}, \overrightarrow{B C}$ in an arrow space $\mathscr{P}_{A}$ we have that

$$
\left\|\overrightarrow{A B}+\left.{ }_{A} \overrightarrow{B C}\right|_{A} \leq\right\| \overrightarrow{A B}\left\|_{A}+\right\| \overrightarrow{B C} \|_{A}
$$

where the equality sign holds if and only if $\overrightarrow{A B}={ }_{A} \overrightarrow{A A}$ ( similarly $\overrightarrow{B C}={ }_{A} \overrightarrow{B B}$ ) or $\overrightarrow{A B}={ }_{A}$ ( $t) \overrightarrow{B C}$ for some $t>0$.

Proof : Notice that by Definitions 6, 5, Equations (1.5), and (1.2) we have

$$
\begin{aligned}
\left\|\overrightarrow{A B}+{ }_{A} \overrightarrow{B C}\right\|_{A}^{2} & =\left\langle\overrightarrow{A B}+{ }_{A} \overrightarrow{B C}, \overrightarrow{A B}+{ }_{A} \overrightarrow{B C}\right\rangle \\
& =\|\overrightarrow{A B}\|_{A}^{2}+2\langle\overrightarrow{A B}, \overrightarrow{B C}\rangle+\|\overrightarrow{B C}\|_{A}^{2}
\end{aligned}
$$

Also, it is always true that

$$
2\langle\overrightarrow{A B}, \overrightarrow{B C}\rangle \leq 2|\langle\overrightarrow{A B}, \overrightarrow{B C}\rangle|
$$


Thus, combining the preceding inequality with Theorem 3.8 yields

$$
2\langle\overrightarrow{A B}, \overrightarrow{B C}\rangle \leq 2\|\overrightarrow{B A}\|_{A}\|\overrightarrow{B C}\|_{A}
$$

So, if we plug in the preceding inequality into the right hand side of Equation (3.60), we get

$$
\left\|\overrightarrow{A B}+{ }_{A} \overrightarrow{B C}\right\|_{A}^{2} \leq\|\overrightarrow{A B}\|_{A}^{2}+2\|\overrightarrow{B A}\|_{A}\|\overrightarrow{B C}\|_{A}+\|\overrightarrow{B C}\|_{A}^{2}
$$

Rearranging the above yields

$$
\left\|\overrightarrow{A B}+{ }_{A} \overrightarrow{B C}\right\|_{A}^{2} \leq\left(\|\overrightarrow{A B}\|_{A}+\|\overrightarrow{B C}\|_{A}\right)^{2} .
$$

Taking the positive square root of both sides of the preceding inequality gives the triangle inequality, that is

$$
\left\|\overrightarrow{A B}+\left.{ }_{A} \overrightarrow{B C}\right|_{A} \leq\right\| \overrightarrow{A B}\left\|_{A}+\right\| \overrightarrow{B C} \|_{A}
$$

Now, we show the equality part of the theorem.

First, suppose that

$$
\left\|\overrightarrow{A B}+\left.{ }_{A} \overrightarrow{B C}\right|_{A}=\right\| \overrightarrow{A B}\left\|_{A}+\right\| \overrightarrow{B C} \|_{A}
$$

Then, we consider the three possible cases as follows.

Case 1. If $A={ }_{P} B$ (similarly if $B={ }_{P} C$ ), we would have $\overrightarrow{A B}={ }_{A} \overrightarrow{A A}$ (similarly $\overrightarrow{B C}={ }_{A}$ $\overrightarrow{B B})$, as desired.

Case 2. If $A={ }_{P} C$, then Equation (3.61) would become

$$
\left\|\overrightarrow{A B}+{ }_{A} \overrightarrow{B A}\right\|_{A}=\|\overrightarrow{A B}\|_{A}+\|\overrightarrow{B A}\|_{A}
$$

By Definition 5 and Equation (1.10), the preceding equation is equivalent to

$$
\|\overrightarrow{A A}\|_{A}=2\|\overrightarrow{A B}\|_{A}
$$

This means by Lemma 1.1 that $\|\overrightarrow{A B}\|_{A}=0$ which means by the same Lemma that $A={ }_{P} B$. But then Definition 3 implies that $\overrightarrow{A B}={ }_{A} \overrightarrow{A A}$, as desired.

Case 3. The last case is when $A, B, C$ are all distinct. Then, by Proposition 3.1 , the Equation (3.61) means that $A \prec B \prec C$. This means by Definition 9 that

$$
\frac{\langle\overrightarrow{B A}, \overrightarrow{B C}\rangle_{A}}{\|\overrightarrow{B A}\|_{A}\|\overrightarrow{B C}\|_{A}}=-1 \text {. }
$$

Then, Part 3 in Theorem 1.10 shows that there exists $s<0$ such that $\overrightarrow{B A}={ }_{A}$ (s) $\overrightarrow{B C}$. This can be rewritten, using Definition 4 , as $\overrightarrow{A B}={ }_{A}(t) \overrightarrow{B C}$, where $t=-s>0$ as $s<0$, as desired. 
Secondly, we show that if $\overrightarrow{A B}={ }_{A} \overrightarrow{A A}$, or if exists $t>0$ such that $\overrightarrow{A B}={ }_{A}(t) \overrightarrow{B C}$, then Equation (3.61) holds. So, let's first consider that $\overrightarrow{A B}={ }_{A} \overrightarrow{A A}$. Then, Definition 3 implies that $B={ }_{P} A$. Thus, we can write with applications of Definition 5 and Lemma 1.1 the following

$$
\left.\left\|\overrightarrow{A B}+\left.{ }_{A} \overrightarrow{B C}\right|_{A}=\right\| \overrightarrow{A C}\right|_{A}=0+\|\overrightarrow{A C}\|_{A}=\|\overrightarrow{A A}\|_{A}+\|\overrightarrow{A C}\|_{A}=\|\overrightarrow{A B}\|_{A}+\|\overrightarrow{B C}\|_{A}
$$

On the other hand, assume that there exists $t>0$ such that $\overrightarrow{A B}={ }_{A}(t) \overrightarrow{B C}$. This can be rewritten, using Definition 4, as

$$
\overrightarrow{B A}={ }_{A}(-t) \overrightarrow{B C}, t>0
$$

Then, the preceding equation with Definition 6, Equations (1.8), and (1.9) lead to

$$
\frac{\langle\overrightarrow{B A}, \overrightarrow{B C}\rangle_{A}}{\|\overrightarrow{B A}\|_{A}\|\overrightarrow{B C}\|_{A}}=\frac{\langle(-t) \overrightarrow{B C}, \overrightarrow{B C}\rangle_{A}}{\|(-t) \overrightarrow{B C}\|_{A}\|\overrightarrow{B C}\|_{A}}=\frac{-t\|\overrightarrow{B C}\|_{A}^{2}}{|t|\|\overrightarrow{B C}\|_{A}\|\overrightarrow{B C}\|_{A}}=-1
$$

By means of Part 2 in Theorem 1.10, the preceding equation shows that

$$
\|\overrightarrow{A C}\|_{A}=\|\overrightarrow{A B}\|_{A}+\|\overrightarrow{B C}\|_{A}
$$

which is equivalent, by Definition 5, to

$$
\left\|\overrightarrow{A B}+\left.{ }_{A} \overrightarrow{B C}\right|_{A}=\right\| \overrightarrow{A B}\left\|_{A}+\right\| \overrightarrow{B C} \|_{A}
$$

That is, Equation (3.61) holds. This ends the proof of this theorem.

\subsection{Pasch's Axiom in Arrow Spaces}

In Section 3.1, we proved the first three axioms of betweenness. In this section, we show that the fourth axiom (Pasch's Axiom) also follows as a theorem in arrow spaces. Since this theorem holds true only in a plane, we need to give a definition of a plane in arrow spaces. The notion of a plane requires the presence of three distinct points that do not lie on the same line. Thus, we need to establish the existence of such points. This can be done in Proposition 3.9 after postulating the following axiom.

Axiom 5. Given two distinct points $A$ and $B$, there exists a point $C$ that is different than $A$ and $B$ such that

$$
\frac{\langle\overrightarrow{A B}, \overrightarrow{A C}\rangle_{A}}{\|\overrightarrow{A B}\|_{A}\|\overrightarrow{A C}\|_{A}} \neq \pm 1
$$

We derive the following proposition using Axiom 5 and Proposition 1.8. 
Proposition 3.9. (The Incidence Axiom I3 in Arrow Spaces) In an arrow space, there exist three distinct points that do not lie on the same line.

Proof : Let $A$ and $B$ be two distinct points in an arrow space. Using Theorem 1.11, consider the line $l_{A B}$. Then, Axiom 5 implies that there exists a third point $C$ such that $C \neq_{P} A, C \neq_{P} B$ and

$$
\frac{\langle\overrightarrow{A B}, \overrightarrow{A C}\rangle_{A}}{\|\overrightarrow{A B}\|_{A}\|\overrightarrow{A C}\|_{A}} \neq \pm 1
$$

An application of Proposition 3.9 to the preceding equation yields that the three points $A, B$, and $C$ do not lie on the same line.

Next, we introduce the following proposition which allows us to rigorously define a plane in arrow spaces.

Proposition 3.10. Let $A, B$ and $C$ be three distinct points in $\mathscr{P}_{A}$ such that $A, B$ and $C$ do not lie on the same line. Let $l_{A B}, l_{A C}$ be the two unique lines that are determined by $\{A, B\},\{A, C\}$, respectively. Then, for any $(s, t) \in \mathbb{R} \times \mathbb{R}$ there exists a unique point $F$ in $\mathscr{P}_{A}$ that corresponds to $(s, t)$. In particular, the point $A$ corresponds to $(0,0)$, points on $l_{A B}, l_{A C}$ correspond to $(0, b),(a, 0), a, b \in \mathbb{R}$, respectively.

Proof : Let $(s, t) \in \mathbb{R} \times \mathbb{R}$. Then we consider the three following cases.

1. If $(s, t)=(0,0)$, then using Definition 7, let $(s) \overrightarrow{A B}={ }_{A} \overrightarrow{A A},(t) \overrightarrow{A C}={ }_{A} \overrightarrow{A A}$. Hence, an application of Theorem 2.2 to $\overrightarrow{A A}$ and $A$ yields that the obtained point is $F={ }_{P} A$ such that $\overrightarrow{A A} \Re \overrightarrow{A A}$.

2. If $s=0, t \neq 0$ (the proof is similar if $s \neq 0, t=0$ ), then using Definition 7, let (s) $\overrightarrow{A B}={ }_{A} \overrightarrow{A A},(t) \overrightarrow{A C}={ }_{A} \overrightarrow{A E}$ for some point $E \in l_{A C}$. Again, an application of Theorem 2.2 to $\overrightarrow{A A}$ and $E$ yields that the obtained point is $F={ }_{P} E$ such that $\overrightarrow{A A} \Re \overrightarrow{E E}$, where $E \in l_{A C}$.

3. Finally, let $(s, t) \in \mathbb{R} \times \mathbb{R}$ be given such that $s \neq 0, t \neq 0$. Consider the arrows $\overrightarrow{A B}$ and $\overrightarrow{A C}$. Then, using Definition 7, let $\overrightarrow{A D}={ }_{A}(s) \overrightarrow{A B}, \overrightarrow{A E}={ }_{A}(t) \overrightarrow{A C}$. An application of Theorem 2.2 to $\overrightarrow{A D}$ and $E$ yields the existence and uniqueness of a point $F$ such that $\overrightarrow{D F} \Re \overrightarrow{A E}$. Note that since $A$ is fixed and by means of Theorem 2.2, the point $F$ is uniquely determined by the two given points $D$ and $E$, which are uniquely determined by $s, t$.

Corollary 3.1. Let $\alpha$ be the set of all points $F$ that are given by Proposition 3.10. Then, there exists a one to one correspondence $\psi: \mathbb{R} \times \mathbb{R}=\{(a, b) \mid a, b \in \mathbb{R}\} \rightarrow \alpha$. 
Proof : Proposition 3.10 implies that there exists a corresponding mapping $\psi: \mathbb{R} \times$ $\mathbb{R} \rightarrow \alpha$, which is given by $\psi(s, t):=F$, where $F$ is the associated point to $(s, t)$ that is given by Proposition 3.10. To show that $\psi$ is one to one, let $(s, t),(p, q) \in \mathbb{R} \times \mathbb{R}$ such that $(s, t) \neq(p, q)$. Let $\psi(s, t)=F, \psi(p, q)=H$, where $F, H$ are given by Proposition 3.10 such that $\overrightarrow{A L}={ }_{A}(s) \overrightarrow{A B}, \overrightarrow{A T}={ }_{A}(t) \overrightarrow{A C}, \overrightarrow{A M}={ }_{A}(p) \overrightarrow{A B}, \overrightarrow{A N}={ }_{A}(q) \overrightarrow{A C}$ and

$$
\overrightarrow{T F} \Re \overrightarrow{A L}, \overrightarrow{N H} \Re \overrightarrow{A M}
$$

as illustrated in Figure 3.4 bellow. We want to show that $\psi(s, t) \neq \psi(p, q)$, that

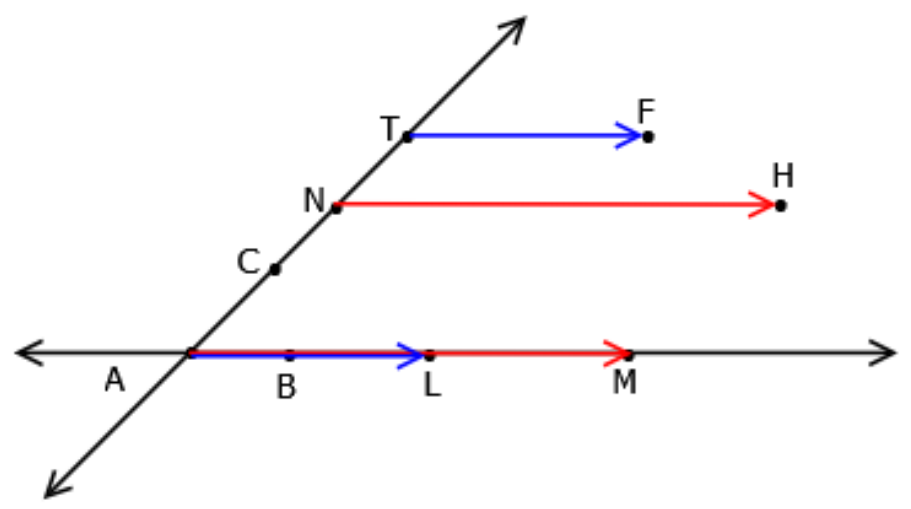

Figure 3.4: Construction of a plane

is, $F \neq_{P} H$. Assume, by way of contradiction, that $F={ }_{p} H$. that is, $\overrightarrow{N F}={ }_{A} \overrightarrow{N H}$. Therefore,

$$
\frac{\langle\overrightarrow{N F}, \overrightarrow{T F}\rangle_{A}}{\|\overrightarrow{N F}\|_{A}\|\overrightarrow{T F}\|_{A}}=\frac{\langle\overrightarrow{N H}, \overrightarrow{T F}\rangle_{A}}{\|\overrightarrow{N H}\|_{A}\|\overrightarrow{T F}\|_{A}} .
$$

Using Axiom (4), the relations in 3.63, and Proposition 1.8 (noticing that $A, L, M \in$ $l_{A B}$ ) we can rewrite the above equation as follows

$$
\frac{\langle\overrightarrow{N F}, \overrightarrow{T F}\rangle_{A}}{\|\overrightarrow{N F}\|_{A}\|\overrightarrow{T F}\|_{A}}=\frac{\langle\overrightarrow{A M}, \overrightarrow{A L}\rangle_{A}}{\|\overrightarrow{A M}\|_{A}\|\overrightarrow{A L}\|_{A}}= \pm 1 .
$$

This means, according to Theorems 1.9 and 1.10, that $N, T, F \in l_{A C}$. Since we have by 3.63 that $\overrightarrow{T F} \Re \overrightarrow{A L}$, it follows by Theorems 1.15 and 2.2 that $L \in l_{A C}$. Thus, we conclude that $A, L \in l_{A B}$ and $A, L \in l_{A C}$, which means by Theorem 1.11 that $l_{A C}=l_{A B}$. But this contradicts the hypothesis of this corollary as the three points $A, B, C$ do not lie on one line. We conclude that our assumption of $F={ }_{P} H$ is impossible and we must have $F \neq_{P} H$. Hence, $\psi$ is one-to-one, as desired. 
We can now define a plane in an arrow space if three distinct points are given such that they do not lie on the same line.

Definition 20. Let $A, B$ and $C$ be three distinct points in $\mathscr{P}_{A}$ such that $A, B$ and $C$ do not lie on the same line. We define a plane that is determined by the three points $A, B$ and $C$ to be the set $\alpha$ that is given by Corollary 3.1.

Corollary 3.2. There exists a one-to-one correspondence between any plane $\alpha$ and the Real Cartesian Plane $\mathbb{R} \times \mathbb{R}$.

Proof : Since we have by Corollary 3.1 that $\psi: \mathbb{R} \times \mathbb{R} \rightarrow \alpha$ is one-to-one and onto, it follows that $\varphi=(\psi)^{-1}: \alpha \rightarrow \mathbb{R} \times \mathbb{R}$ is also one-to-one and onto.

Theorem 3.1. (Pasch's Axiom, The Axiom B4 of Betweenness) Let A, B and C be three distinct points in a plane $\alpha$ such that these points do not lie on a line, and let $l$ be a line not containing any of $A, B$ and $C$. If l contains a point $D$ lying between $A$ and $B$, then it must also contain either a point lying between $A$ and $C$ or a point lying between $B$ and $C$.

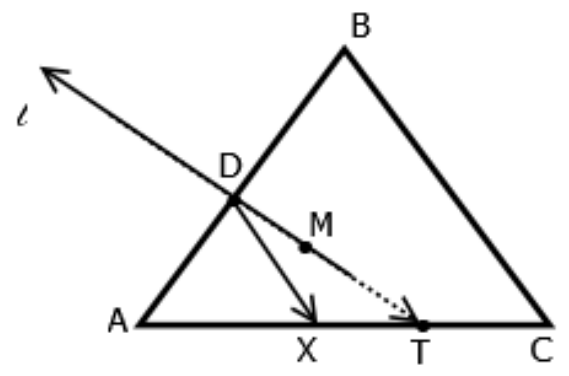

Figure 3.5: Pasch's Axiom

Proof : Consider the set of all points $X$ that lie between $A, C$ or between $B, C$. Let $s=\|\overrightarrow{B X}\|_{A}$. Then, since $X$ is either between $B, C$ or between $A, C$, we either have (by betweennes) $s \leq\|\overrightarrow{B C}\|_{A}$ or $s \leq\|\overrightarrow{B C}\|_{A}$ (by betweenness and Theorem 3.9), respectively. Thus, we have that

$$
0 \leq s \leq\|\overrightarrow{B C}\|_{A}+\|\overrightarrow{C A}\|_{A}
$$

Put $\theta_{X}:=\angle B D X$. Then, by Theorem 3.7 we have the following: as $\theta_{X}$ varies between

$$
\begin{gathered}
\theta_{X}=0 \Longleftrightarrow X={ }_{p} B \text { to } \theta_{X}=\pi \Longleftrightarrow X={ }_{P} A, \\
-1 \leq \cos \theta_{X}=\frac{\langle\overrightarrow{D B}, \overrightarrow{D X}\rangle_{A}}{\|\overrightarrow{D B}\|_{A}\|\overrightarrow{D X}\|_{A}} \leq 1
\end{gathered}
$$


Consider the point $D$ as a point of separation of the line $l$. Let $M$ be a point on $l$ other than $D$ (since the cosine of vertical angle are the same, we may pick $M$ in a suitable way) and put $\psi=\angle M D B$. Then, we have that $0 \leq \psi \leq \pi$. Thus, by continuity of the strictly monotone function $\cos \theta_{x}$ as $X$ varies, there exists a unique $0 \leq \theta_{X}=\psi \leq \pi$ that corresponds to a unique point $X={ }_{P} T$.

\subsection{Plane Separation Theorem and Convex sets in Ar- row Spaces}

As an application of Theorem 3.1 (Pasch's Axiom) in arrow spaces, we prove the so called Plane Separation Theorem. This theorem brings in the notion of dividing a plane into two sides. Having established this notion early on, we are later able to state Theorem 3.6 (a congruence axiom about laying off an angle in a given side of a line). We follow the proof that Hartshorne provides in [20] page 74, but in order for the proof to be consistent with our constructions in arrow spaces, we make some changes to the definition of the equivalence relation used in [20].

Theorem 3.10. (The Plane Separation Theorem In Arrow Spaces)

Let $\alpha$ be a plane and $l$ be a line in $\alpha$. Then, the set of points not lying on l can be divided into two non-empty sets $S_{1}, S_{2}$ with the following properties:

1-Two points $A, B$ not on l belong to the same set $\left(S_{1}\right.$ or $\left.S_{2}\right)$ if and only if for each $t \in(0,1)$ and each $D \in \alpha$ with $\overrightarrow{A D}={ }_{A}(t) \overrightarrow{A B}$ we must have $D \notin l$.

2-Two points $A, C$ not on l belong to the opposite sets (one in $S_{1}$ and the other in $\left.S_{2}\right)$ if and only if there exists $t_{0} \in(0,1)$ and $D_{0} \in \alpha$ with $\overrightarrow{A D_{0}}={ }_{A}\left(t_{0}\right) \overrightarrow{A B}$ and $D_{0} \in l$.

Proof : We define a relation $\sim$ on the set $\alpha \backslash l$ as follows: $A \sim B$ if either $A={ }_{P} B$ or for any $D \in \alpha$ and for any $t \in(0,1)$ with $\overrightarrow{A D}={ }_{A}(t) \overrightarrow{A B}$ we have $D \notin l$. We show that $\sim$ is an equivalence relation.

1. First, $\sim$ is reflexive since for each point $A \in \alpha \backslash l$ we have $A={ }_{P} A$ and so $A \sim A$.

2. We show that $\sim$ is symmetric. Let $A \sim B$. If $A={ }_{P} B$, then $B={ }_{P} A$ and by the definition of $\sim$ we have $B \sim A$. If $A \neq \neq_{P} B$, then let $t \in(0,1)$ and $D \in \alpha$ be such that

$$
\overrightarrow{B D}={ }_{A}(t) \overrightarrow{B A}, t \in(0,1)
$$

To show that $B \sim A$ we have to show that $D \notin l$. Since $t \in(0,1)$, it follows that 
$1-t \in(0,1)$. Now, we have by Equations (1.5), (1.6), and Definitions 5, 4 that

$$
\begin{aligned}
\|\overrightarrow{A D}\|_{A}^{2} & =\langle\overrightarrow{A D}, \overrightarrow{A D}\rangle_{A}=\left\langle\overrightarrow{A B}+_{A} \overrightarrow{B D}, \overrightarrow{A B}+_{A} \overrightarrow{B D}\right\rangle_{A} \\
& =\langle\overrightarrow{A B}, \overrightarrow{A B}\rangle_{A}+\left\langle\overrightarrow{A B}+_{A} \overrightarrow{B D}\right\rangle_{A}+\langle\overrightarrow{B D}, \overrightarrow{A B}\rangle_{A}+\langle\overrightarrow{B D}, \overrightarrow{B D}\rangle_{A} \\
& =\|\overrightarrow{A B}\|_{A}^{2}+2\langle\overrightarrow{A B}, \overrightarrow{B D}\rangle_{A}+\|\overrightarrow{B D}\|_{A}^{2} \\
& =\|\overrightarrow{A B}\|_{A}^{2}-2 t\langle\overrightarrow{A B}, \overrightarrow{A B}\rangle_{A}+t^{2}\|\overrightarrow{A B}\|_{A}^{2} \\
& =\left(1-2 t+t^{2}\right)\|\overrightarrow{A B}\|_{A}^{2} \\
& =(1-t)^{2}\|\overrightarrow{A B}\|_{A}^{2} .
\end{aligned}
$$

Taking the positive square root of both sides of the above yields

$$
\|\overrightarrow{A D}\|_{A}=(1-t)\|\overrightarrow{A B}\|_{A}
$$

This is equivalent, by Equation (1.9), to (notice that $1-t \in(0,1)$ )

$$
\|\overrightarrow{A D}\|_{A}=\|(1-t) \overrightarrow{A B}\|_{A}
$$

We also have by Equation (1.8) and since $(1-t)>0$ that

$$
\frac{\langle\overrightarrow{A D},(1-t) \overrightarrow{A B}\rangle_{A}}{\|\overrightarrow{A D}\|_{A}\|(1-t) \overrightarrow{A B}\|_{A}}=\frac{(1-t)\langle\overrightarrow{A D}, \overrightarrow{A B}\rangle_{A}}{(1-t)\|\overrightarrow{A D}\|_{A}\|\overrightarrow{A B}\|_{A}}=1
$$

It follows then by Equations (3.67), (3.68), and Proposition 1.5 that $\overrightarrow{A D}={ }_{A}$ $(1-t) \overrightarrow{A B}$. Then, since $(1-t) \in(0,1)$ and $A \sim B$ it follows by the definition of $\sim$ in this theorem that we must have $D \notin l$ which means by Equation (3.66) and the definition of $\sim$ again that $B \sim A$, as desired.

3. Finally, we prove that $\sim$ is transitive. So, let $A, B$, and $C$ be three points in $\alpha \backslash l$. If at least two of the three points are equal, then the transitivity of $\sim$ is immediate. This is because if we have, for example, $A={ }_{P} B$, then $A \sim B$ and $B \sim C$ would imply that $A \sim C$ as $A={ }_{P} B$. Now, let the three points be distinct such that

$$
A \sim B \text { and } B \sim C .
$$

We want to show that $A \sim C$. So, let

$$
\left.t \in(0,1) \text { and } D \in \alpha \backslash l \text {, with } \overrightarrow{A D}=_{A}(t) \overrightarrow{A C} \text { (that is } A \prec D \prec C\right) \text {. }
$$


Now, we have two possible cases regarding the three points $A, B$, and $C$ being on a line.

Case (1). The three points $A, B$, and $C$ do not lie on one line. In this case assume, by way of contradiction, that $D$ in Equation (3.69) lies on the line $l$. Now, since $A \sim B$ and $B \sim C$, then by the definition of $\sim$, we have $A, B, C \notin l$. By means of Theorem $3.1, \overrightarrow{A D}={ }_{A}(t) \overrightarrow{A C}$ with $t \in(0,1)$ implies that $D$ is between $A$ and $C$. Therefore, if $D \in l$, we would have by Theorem 3.1 that the line $l$ must contain a point, say $D^{\prime}$, which lies either between $A$ and $B$ or between $B$ and $C$, see Figure 3.6. But then this would mean that there exists $t^{\prime} \in(0,1)$ such that $\overrightarrow{A D^{\prime}}={ }_{A}\left(t^{\prime}\right) \overrightarrow{A B}$ or $\overrightarrow{B D^{\prime}}={ }_{A}\left(t^{\prime}\right) \overrightarrow{B C}$. Each of the preceding cases is impossible since each of the relations $A \sim B$ and $B \sim C$ implies that $D^{\prime} \notin l$, a contradiction. Therefore, our assumption $D \in l$ is false and we must have $D \notin l$ which means by Equation (3.70) and the definition of $\sim$ that $A \sim C$.

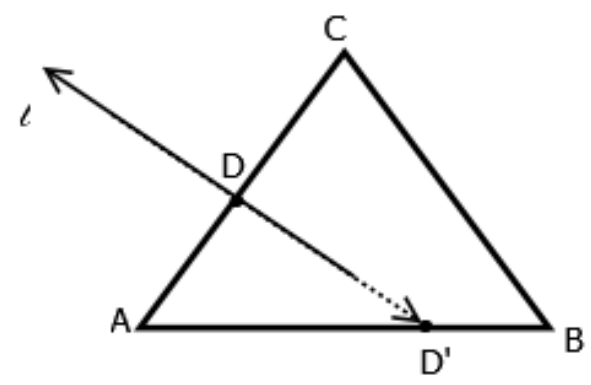

Figure 3.6: An illustration of Case 1 where the points $A, B$, and $C$ are not one the same line.

Case (2). The three points lie on the same line, say $l_{A B}$. Then, we also have another two cases, but this time regarding the betweenness. In particular, if the point $C$ lies between $A$ and $B$, then it follows by Theorem 3.2 that there exists $s \in(0,1)$ such that

$$
\overrightarrow{A C}={ }_{A}(s) \overrightarrow{A B}, s \in(0,1) .
$$

Then, we have by Equations (3.70), (3.71), and Theorem 1.5 that

$$
\overrightarrow{A D}={ }_{A}(t)(\overrightarrow{A C})={ }_{A}(t)((s) \overrightarrow{A B})=(t s) \overrightarrow{A B}, t s \in(0,1)
$$

But since we have by Equation (3.69) that $(A \sim B)$, it follows then by the definition of the relation $\sim$ that $D \notin l$. Combining this with Equation (3.70) and the definition of the relation $\sim$ implies that $A \sim C$, as desired.

On the other hand, if $C$ is not between $A$ and $B$, then we have by Theorem 3.3 that either $A$ or $B$ lies between the other two. So assume, without loss of 
generality, that

$$
A \prec B \prec C .
$$

This means by Theorem 3.2 that there exists $s \in(0,1)$ such that

$$
\overrightarrow{A B}={ }_{A}(s) \overrightarrow{A C}, s \in(0,1) .
$$

It follows by Equations (3.70), (3.74), and (1.9) that (notice that $t, s>0$ )

$$
\|\overrightarrow{A D}\|_{A}=\|(t) \overrightarrow{A C}\|_{A}=\frac{t}{s}\|(s) \overrightarrow{A C}\|_{A}=\frac{t}{s}\|\overrightarrow{A B}\|_{A}=\left\|\left(\frac{t}{s}\right) \overrightarrow{A B}\right\|_{A} .
$$

Also, we have by Equations (3.70), (3.74), (1.9), and (1.8) that

$$
\frac{\langle\overrightarrow{A D}, \overrightarrow{A B}\rangle_{A}}{\|\overrightarrow{A D}\|_{A}\|\overrightarrow{A B}\|_{A}}=\frac{\langle(t) \overrightarrow{A C},(s) \overrightarrow{A C}\rangle_{A}}{\|(t) \overrightarrow{A C}\|_{A}\|(s) \overrightarrow{A C}\|_{A}}=\frac{(t s)\langle\overrightarrow{A C}, \overrightarrow{A C}\rangle}{(t s)\|\overrightarrow{A C}\|_{A}^{2}}
$$

This simplifies by Definition 6 to

$$
\frac{\langle\overrightarrow{A D}, \overrightarrow{A B}\rangle_{A}}{\|\overrightarrow{A D}\|_{A}\|\overrightarrow{A B}\|_{A}}=\frac{\|\overrightarrow{A C}\|^{2}}{\|\overrightarrow{A C}\|_{A}^{2}}=1
$$

Thus, since $\frac{t}{s}>0$ the preceding equation and by 1.9 we have

$$
\frac{\left\langle\overrightarrow{A D},\left(\frac{t}{s}\right) \overrightarrow{A B}\right\rangle_{A}}{\|\overrightarrow{A D}\|_{A}\left\|\left(\frac{t}{s}\right) \overrightarrow{A B}\right\|_{A}}=\frac{\frac{t}{s}\langle\overrightarrow{A D}, \overrightarrow{A B}\rangle_{A}}{\frac{t}{s}\|\overrightarrow{A D}\|_{A}\|\overrightarrow{A B}\|_{A}}=1 .
$$

It follows now by Equations (3.75), (3.77), and Proposition 1.5 that

$$
\overrightarrow{A D}={ }_{A}\left(\frac{t}{s}\right) \overrightarrow{A B}
$$

Now, if $t<s$, then since $s, t>0$, we have $0<\frac{t}{s}<1$. Then, since $A \sim B$, Equation (3.78) and the definition of $\sim$ mean that $D \notin l$. Hence, Equation (3.70) with the definition of $\sim$ implies that $A \sim C$, as desired. The other possibility is that $s<t$, that is, $0<\frac{s}{t}<1$ as $s, t>0$. Now, by Equations (3.70), (3.74), and (1.9) we have

$$
\begin{aligned}
\|\overrightarrow{A B}\|_{A}=\|(s) \overrightarrow{A C}\|_{A} & =s\|\overrightarrow{A C}\|_{A}=\frac{s}{t}\|(t) \overrightarrow{A C}\|_{A} \\
& =\frac{s}{t}\|\overrightarrow{A D}\|_{A}=\left\|\left(\frac{s}{t}\right) \overrightarrow{A D}\right\|_{A} .
\end{aligned}
$$


Thus, since $\frac{s}{t}>0$ we have by Equations (3.76) and (1.9) that

$$
\frac{\left\langle\overrightarrow{A D},\left(\frac{s}{t}\right) \overrightarrow{A B}\right\rangle_{A}}{\|\overrightarrow{A D}\|_{A}\left\|\left(\frac{s}{t}\right) \overrightarrow{A B}\right\|_{A}}=\frac{\frac{s}{t}\langle\overrightarrow{A D}, \overrightarrow{A B}\rangle_{A}}{\frac{s}{t}\|\overrightarrow{A D}\|_{A}\|\overrightarrow{A B}\|_{A}}=1
$$

It follows now by Equations (3.75), (3.80), and Proposition 1.5 that

$$
\overrightarrow{A B}={ }_{A}\left(\frac{s}{t}\right) \overrightarrow{A D}
$$

but since $0<\frac{s}{t}<1$, the preceding equation means by Theorem 3.1

$$
A \prec B \prec D \text {. }
$$

Notice that, the current argument, that is the possibility of $s<t$, includes the following

$$
A \prec D \prec C, A \prec B \prec C \text {, and } A \prec B \prec D,
$$

that follow from the relations in (3.70), (3.73), and (3.82), respectively. By means of Proposition 3.2 the preceding equation implies that

$$
B \prec D \prec C .
$$

But since $B \sim C$, we will have by the definition of $\sim$ that $D \notin l$. This means by the definition of $\sim$ again and Equation (3.70) that $A \sim C$. Therefore, the relation $\sim$ is transitive; and so is equivalence relation.

This means that $\sim$ divides the set $\alpha \backslash l$ into a disjoint union of equivalence classes, and these equivalence classes will satisfy Part 1 of this theorem by definition. To finish the proof of this theorem, it is enough to show that there are exactly two equivalence classes $S_{1}, S_{2}$ for the relation $\sim$. Then, to say that there exists $t \in(0,1)$ and $D \in \alpha$ such that $\overrightarrow{A D}={ }_{A}(t) \overrightarrow{A C}$, which is equivalent to $A \nsim C$, will be the same as saying that $A, C$ belong to the opposite sets. Now, let $D \in \alpha \backslash l$. Then, this means that there is at least one equivalence class $S_{1}$. Given a point $A \in S_{1}$, let $D$ be any point on $l$ and use Theorem 3.4 to get a point $C$ such that

$$
\overrightarrow{A D}={ }_{A}(t) \overrightarrow{A C}, t \in(0,1)
$$

This means by the definition of the relation $\sim$ that $A \nsim C$. Thus, there must be at least two equivalence classes $S_{1}, S_{2}$. Finally, we show that there are at most two equivalence classes, that is, we show that if

$$
A \nsim C \text { and } B \nsim C \text {, then } A \sim B .
$$

There are two possible cases regarding the points $A, B$, and $C$ as follows. 
1. If $A, B$, and $C$ do not lie on one line, then since $A \nsim C$, we conclude that there exists a point on $l$ that is between $A$ and $C$. Also, since $B \nsim C$ we conclude that there exists a point on $l$ that is between $B$ and $C$. This means, by Theorem 3.1 that any point between $A$ and $B$ does not lie on $l$ which means by the definition of $\sim$ that $A \sim B$, as desired.

2. Consider the case in which $A, B$, and $C$ lie on one line, say $l_{A B}$. Then, let $D$ be any point on $l$ with $D \notin l_{A B}$ and use Theorem 3.4 to get a point $E$ such that

$$
\overrightarrow{D A}={ }_{A}(t) \overrightarrow{D E}, t \in(0,1)
$$

Then, we have that $A \sim E$. Now, since by hypothesis we have $A \nsim C$, and since we just got $A \sim E$, we conclude that $C \nsim E$ because the relation $\sim$ is transitive. Looking at the three points $B, C$, and $E$ that do not lie on one line, from $E \nsim C$ and $B \nsim C$, we conclude using case 1 that $B \sim E$. But, also $A \sim E$, so by the transitivity of the relation $\sim$, we have $A \sim B$, as desired.

Next, we state a definition of a convex set in terms of arrows.

Definition 21. Let $S$ be a nonempty set of points. We say that $S$ is a convex set if for any two distinct points $A, B \in S$, the following holds true

$$
\overrightarrow{A P}={ }_{A}(t) \overrightarrow{A B}, t \in(0,1) \text { implies } P \in S .
$$

Now, we prove that the two sets, $S_{1}, S_{2}$ in Theorem 3.10 are convex sets.

Proposition 3.11. Each of the two sets in The Plane Separation Theorem, namely $S_{1}, S_{2}$, is a convex set.

Proof : We prove the proposition for $S_{1}$ and the proof for $S_{2}$ should be the same. Let $A, B \in S_{1}$ and let $C$ be any point between $B$ and $A$. This means by Theorem 3.2 that there exists $t \in(0,1)$ such that

$$
\overrightarrow{B C}={ }_{A}(t) \overrightarrow{B A}, t \in(0,1)
$$

Assume, by way of contradiction, that $C \in S_{2}$. Then, since $B \in S_{1}$, this would mean that $B$ and $C$ are in different sides of $l$. It follows by the definition of the relation in Theorem 3.10 that there exists $s \in(0,1)$ and a point $D$ on $l$ such that

$$
\overrightarrow{B D}={ }_{A}(s) \overrightarrow{B C}, s \in(0,1)
$$

Combining Equations (3.89) and (3.90) and an application of Theorem 1.5 give

$$
\overrightarrow{B D}={ }_{A}(s) \overrightarrow{B C}={ }_{A}(s)((t) \overrightarrow{B A})={ }_{A}(s t)(\overrightarrow{B A})
$$


But since $s, t \in(0,1)$, it is also true that $s t \in(0,1)$. Then, Equation (3.91) together with the definition of $\sim$ would mean that $A \nsim B$ as $D \in l$. This is a contradiction to the hypothesis that $A, B \in S_{1}$ which means $A \sim B$. Therefore, the assumption that a point between $A, B \in S_{1}$ may be in $S_{2}$ is false. This means that if $A, B \in S_{1}$, then every point between $A B$ must be in $S_{1}$, too. Hence, Definition 21 implies that $S_{1}$ is a convex set.

Remark 3.3. Using our setting from this section and Section 3.3, we associate with each angle between two rays $\overrightarrow{A B}_{\infty}$ and $\overrightarrow{A C}_{\infty}$, that emanate from the same point, a convex set as follows. First, if $A, B$ and $C$ lie on the line $l_{A B}$, then this convex set would be the angle itself, that is the union of the two rays which is a convex set.

Secondly, if the points $A, B$ and $C$ do not lie on a line, then the following theorem describes the convex set that is associated with the two rays $\overrightarrow{A B}_{\infty}$ and $\overrightarrow{A C}_{\infty}$.

Theorem 3.11. Let $A, B$ and $C$ be three distinct points that do not lie on a line. Consider the two lines $l_{A B}, l_{A C}$. Let $S_{1}, S_{2}$ and $S_{1}^{\prime}, S_{2}^{\prime}$ be the two pairs of convex sets that we obtain from Theorem 3.10, and are associated with $l_{A B}, l_{A C}$, respectively, see Figure 3.7 and 3.8. If $C \in S_{1}$ and $B \in S_{1}^{\prime}$, then $S_{1} \cap S_{1}^{\prime}$ is nonempty and convex set, see Figure 3.9.

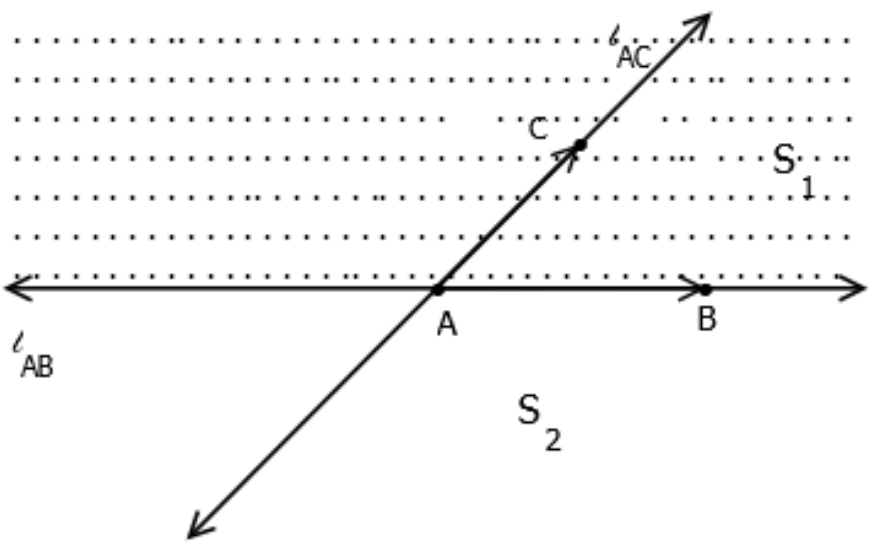

Figure 3.7: The convex set $S_{1}$, with respect to the line $l_{A B}$, that contains the point $C$. 


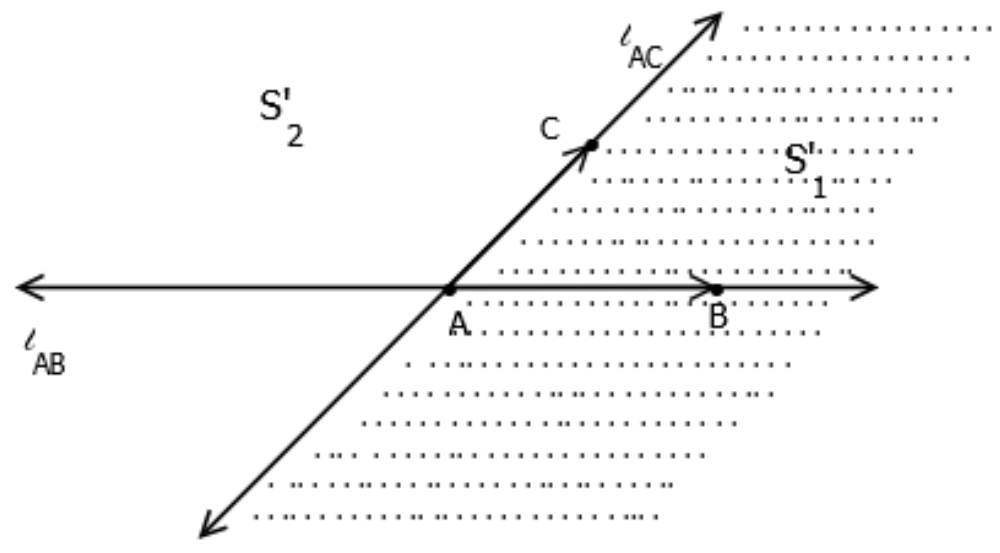

Figure 3.8: The convex set $S_{1}^{\prime}$, with respect to the line $l_{A C}$, that contains the point $B$.

Proof : Notice that, since $B \notin l_{A C}$ and $C \notin l_{A B}$ it follows by Theorem 3.10 that $B \in S_{1}^{\prime}$ or $B \in S_{2}^{\prime}$ and also either $C \in S_{1}$ or $C \in S_{2}$. So assume, without lose of generality, that $B \in S_{1}^{\prime}$ and $C \in S_{1}$. Then, set $S_{1} \cap S_{1}^{\prime}$ is nonempty because at least it contains all points that lie between $B, C$. Indeed, if $P$ is any points between $B, C$, then we have by Theorem 3.3 that $C$ cannot be between $B$ and $P$. Thus, since $C \in l_{A C}$ it follows by the definition of the relation $\sim$ in Theorem 3.10 that $P \sim B$, that is, $P \in S_{1}$. Similarly, $P \in S_{1}^{\prime}$; and so $P \in\left(S_{1} \cap S_{1}^{\prime}\right)$. On the other hand, the set $S_{1} \cap S_{1}^{\prime}$ is convex as by Proposition 3.11 each of the sets $S_{1}$ and $S_{2}$ is convex. Since the intersection of finite number of convex sets is a convex set, we conclude that $S_{1} \cap S_{1}^{\prime}$ is convex.

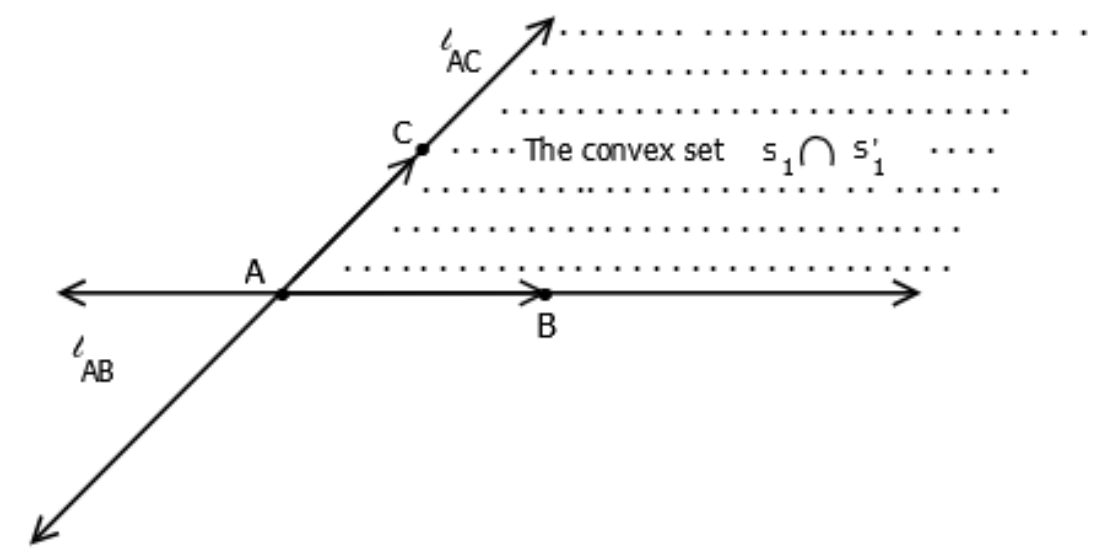

Figure 3.9: An illustration of the convex set that is associated with an angle $\angle C A B$ between two rays. 


\subsection{Line Circle Intersection Property in Arrow Spaces}

Our next mission in arrow spaces is to prove the well known geometrical property of Line-Circle Intersection. Hartshorne shows in [20] that in the Cartesian plane over an ordered field, this property is equivalent to the Circle-Circle intersection property. In order to prove the Line-Circle Intersection Property in an arrow space, we need to give a definition of a circle.

Definition 22. Let $C, A$ be any two distinct points. We define a circle, denoted by $\mathscr{C}$, with center $C$ and a radius $r=\|\overrightarrow{C A}\|_{A}>0$ to be the set $\mathscr{C}=\left\{P\|\| \overrightarrow{C P} \|_{A}=r\right\}$. If $\|\overrightarrow{C N}\|_{A}<r$ for some point $N$, we say that the point $N$ is inside the circle $\mathscr{C}$ and we say that a point $M$ is outside $\mathscr{C}$ if $\|\overrightarrow{C M}\|_{A}>r$, see Figure 3.10 .
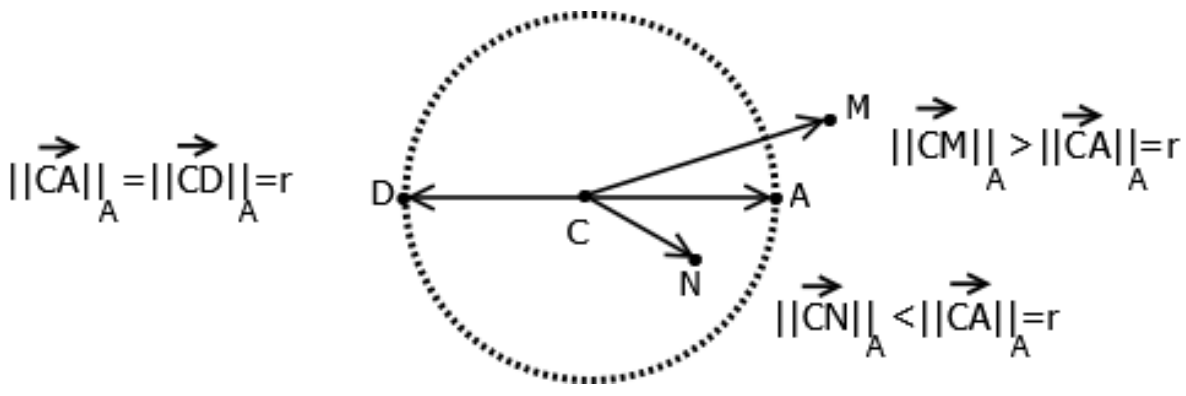

Figure 3.10: A Circle

Proposition 3.12. Let $\mathscr{C}$ be a circle with a center $C$ and a radius $r$. Then, $C$ is inside the circle $\mathscr{C}$.

Proof : Notice that, by Lemma 1.1 we have $\|\overrightarrow{C C}\|_{A}=0<r$ which means by Definition 22 that $C$ is inside the circle $\mathscr{C}$.

Now, we show that if a line has a point inside and a point outside a circle, then this line meets the circle in two points. To prove this property, we need the following Lemma.

Lemma 3.2. Let $\mathscr{C}$ be a circle with a center $C$ and a radius $r$. Let $N, M$ be two distinct points with $\|\overrightarrow{C N}\|_{A}<r$ and $\|\overrightarrow{C M}\|_{A}>r$. Then, for any point $D$ such that $\|\overrightarrow{C D}\|_{A}=$ rwe must have

$$
\left(\langle\overrightarrow{C M}, \overrightarrow{M N}\rangle_{A}\right)^{2}-\|\overrightarrow{M N}\|_{A}^{2}\left(\|\overrightarrow{C M}\|_{A}^{2}-\|\overrightarrow{C D}\|_{A}^{2}\right)>0
$$

Proof : Let $N, M$ be two distinct points with $\|\overrightarrow{C N}\|_{A}<r$ and $\|\overrightarrow{C M}\|_{A}>r$ where $r$ is a radius of a circle $\mathscr{C}$ with a center $C$. Let $D$ be any point such that $\|\overrightarrow{C D}\|_{A}=r$, see Figure 3.10. It follows that

$$
\|\overrightarrow{C N}\|_{A}<\|\overrightarrow{C D}\|_{A}
$$


Now, we have by Equation (1.5), (1.6), and Definitions 5, 4 that

$$
\begin{aligned}
\left(\langle\overrightarrow{C M}, \overrightarrow{M N}\rangle_{A}\right)^{2} & =\left(\langle\overrightarrow{C M}, \overrightarrow{M N}\rangle_{A}\right)\left(\langle\overrightarrow{C M}, \overrightarrow{M N}\rangle_{A}\right) \\
& =\left(\left\langle\overrightarrow{C N}+{ }_{A} \overrightarrow{N M}, \overrightarrow{M N}\right\rangle_{A}\right)\left(\left\langle\overrightarrow{C M}, \overrightarrow{M C}+{ }_{A} \overrightarrow{C N}\right\rangle_{A}\right) \\
& =\left[\langle\overrightarrow{C N}, \overrightarrow{M N}\rangle_{A}-\langle\overrightarrow{M N}, \overrightarrow{M N}\rangle_{A}\right]\left[\langle\overrightarrow{C M}, \overrightarrow{C N}\rangle_{A}-\right. \\
& \left.\langle\overrightarrow{C M}, \overrightarrow{C M}\rangle_{A}\right] .
\end{aligned}
$$

The above equation simplifies by Definition 6 to

$$
\begin{aligned}
\left(\langle\overrightarrow{C M}, \overrightarrow{M N}\rangle_{A}\right)^{2} & =\langle\overrightarrow{C N}, \overrightarrow{M N}\rangle_{A}\langle\overrightarrow{C M}, \overrightarrow{C N}\rangle_{A}- \\
& \langle\overrightarrow{C N}, \overrightarrow{M N}\rangle_{A}\|\overrightarrow{C M}\|_{A}^{2}-\left(\|\overrightarrow{M N}\|_{A}^{2}\langle\overrightarrow{C M}, \overrightarrow{C N}\rangle_{A}+\right. \\
& \|\overrightarrow{M N}\|_{A}^{2}\|\overrightarrow{C M}\|_{A}^{2} .
\end{aligned}
$$

If we combine the first and second terms in the right hand side of the above equation by using Equation (1.5) and Definitions 6, 5, we get

$$
\begin{aligned}
\left(\langle\overrightarrow{C M}, \overrightarrow{M N}\rangle_{A}\right)^{2} & =\langle\overrightarrow{C N}, \overrightarrow{M N}\rangle_{A}\left(\langle\overrightarrow{C M}, \overrightarrow{C N}\rangle_{A}+\langle\overrightarrow{C M}, \overrightarrow{M C}\rangle_{A}\right)- \\
& \|\overrightarrow{M N}\|_{A}^{2}\langle\overrightarrow{C M}, \overrightarrow{C N}\rangle_{A}+\|\overrightarrow{M N}\|_{A}^{2}\|\overrightarrow{C M}\|_{A}^{2} \\
& =\langle\overrightarrow{C N}, \overrightarrow{M N}\rangle_{A}\langle\overrightarrow{C M}, \overrightarrow{M N}\rangle_{A}- \\
& \|\overrightarrow{M N}\|_{A}^{2}\langle\overrightarrow{C M}, \overrightarrow{C N}\rangle_{A}+\|\overrightarrow{M N}\|_{A}^{2}\|\overrightarrow{C M}\|_{A}^{2}
\end{aligned}
$$

Now, we try to do the same thing with the term $\|\overrightarrow{M N}\|_{A}^{2}\|\overrightarrow{C M}\|_{A}^{2}$. By Equations (1.5), (1.6), and Definitions 6, 5, and 4 we can write

$$
\begin{aligned}
\|\overrightarrow{M N}\|_{A}^{2}\|\overrightarrow{C M}\|_{A}^{2} & =\langle\overrightarrow{M N}, \overrightarrow{M N}\rangle_{A}\langle\overrightarrow{C M}, \overrightarrow{C M}\rangle_{A} \\
& =\left\langle\overrightarrow{M C}+{ }_{A} \overrightarrow{C N}, \overrightarrow{M N}\right\rangle_{A}\left\langle\overrightarrow{C M}, \overrightarrow{C N}+_{A} \overrightarrow{N M}\right\rangle_{A} \\
& =\left[\langle\overrightarrow{M C}, \overrightarrow{M N}\rangle_{A}+\langle\overrightarrow{C N}, \overrightarrow{M N}\rangle_{A}\right]\left[\langle\overrightarrow{C M}, \overrightarrow{C N}\rangle_{A}\right. \\
& \left.+\langle\overrightarrow{C M}, \overrightarrow{N M}\rangle_{A}\right] \\
& =\left(\langle\overrightarrow{M C}, \overrightarrow{M N}\rangle_{A}\langle\overrightarrow{C M}, \overrightarrow{C N}\rangle_{A}\right)+\left(\langle\overrightarrow{C M}, \overrightarrow{M N}\rangle_{A}\right)^{2}+ \\
& \left(\langle\overrightarrow{C N}, \overrightarrow{M N}\rangle_{A}\langle\overrightarrow{C M}, \overrightarrow{C N}\rangle_{A}\right)+ \\
& \left(\langle\overrightarrow{C N}, \overrightarrow{M N}\rangle_{A}\langle\overrightarrow{C M}, \overrightarrow{N M}\rangle_{A}\right) .
\end{aligned}
$$


Combining the first and third terms in the right hand side of the above and using Definitions 5, 6, and Equation (1.5) yields

$$
\begin{aligned}
\|\overrightarrow{M N}\|_{A}^{2}\|\overrightarrow{C M}\|_{A}^{2}= & \|\overrightarrow{M N}\|_{A}^{2}\langle\overrightarrow{C M}, \overrightarrow{C N}\rangle_{A}+\left(\langle\overrightarrow{C M}, \overrightarrow{M N}\rangle_{A}\right)^{2}+ \\
& \langle\overrightarrow{C N}, \overrightarrow{M N}\rangle_{A}\langle\overrightarrow{C M}, \overrightarrow{N M}\rangle_{A}
\end{aligned}
$$

Applying Equation (1.5) and Definition 5 again gives

$$
\begin{aligned}
\|\overrightarrow{M N}\|_{A}^{2}\|\overrightarrow{C M}\|_{A}^{2} & =\|\overrightarrow{M N}\|_{A}^{2}\langle\overrightarrow{C M}, \overrightarrow{C N}\rangle_{A}+\left(\langle\overrightarrow{C M}, \overrightarrow{M N}\rangle_{A}\right)^{2}+ \\
& \langle\overrightarrow{C N}, \overrightarrow{M N}\rangle_{A}\left(\langle\overrightarrow{C N}, \overrightarrow{N M}\rangle_{A}+\langle\overrightarrow{N M}, \overrightarrow{N M}\rangle_{A}\right) .
\end{aligned}
$$

If we multiply out the last terms and use Definition 6, we get

$$
\begin{aligned}
\|\overrightarrow{M N}\|_{A}^{2}\|\overrightarrow{C M}\|_{A}^{2} & =\|\overrightarrow{M N}\|_{A}^{2}\langle\overrightarrow{C M}, \overrightarrow{C N}\rangle_{A}+\left(\langle\overrightarrow{C M}, \overrightarrow{M N}\rangle_{A}\right)^{2}+ \\
& \langle\overrightarrow{C N}, \overrightarrow{M N}\rangle_{A}\langle\overrightarrow{C N}, \overrightarrow{N M}\rangle_{A}+\langle\overrightarrow{C N}, \overrightarrow{M N}\rangle_{A}\|\overrightarrow{M N}\|_{A}^{2}
\end{aligned}
$$

Rearranging the preceding equation with use of Equation (1.5) and Definitions 5 and 6 gives

$$
\begin{aligned}
\|\overrightarrow{M N}\|_{A}^{2}\|\overrightarrow{C M}\|_{A}^{2} & =\|\overrightarrow{M N}\|_{A}^{2}\left(\langle\overrightarrow{C M}, \overrightarrow{C N}\rangle_{A}+\langle\overrightarrow{C N}, \overrightarrow{M N}\rangle_{A}\right)+ \\
& \left(\langle\overrightarrow{C M}, \overrightarrow{M N}\rangle_{A}\right)^{2}-\left(\langle\overrightarrow{C N}, \overrightarrow{M N}\rangle_{A}\right)^{2} \\
& =\|\overrightarrow{M N}\|_{A}^{2}\|\overrightarrow{C N}\|_{A}^{2}+\left(\langle\overrightarrow{C M}, \overrightarrow{M N}\rangle_{A}\right)^{2}-\left(\langle\overrightarrow{C N}, \overrightarrow{M N}\rangle_{A}\right)^{2}
\end{aligned}
$$

If we subtract Equation (3.95) from Equation (3.94), we get

$$
\begin{aligned}
\left(\langle\overrightarrow{C M}, \overrightarrow{M N}\rangle_{A}\right)^{2}-\|\overrightarrow{M N}\|_{A}^{2}\|\overrightarrow{C M}\|_{A}^{2} & =\langle\overrightarrow{C N}, \overrightarrow{M N}\rangle_{A}\langle\overrightarrow{C M}, \overrightarrow{M N}\rangle_{A}- \\
& \|\overrightarrow{M N}\|_{A}^{2}\langle\overrightarrow{C M}, \overrightarrow{C N}\rangle_{A}+\|\overrightarrow{M N}\|_{A}^{2}\|\overrightarrow{C M}\|_{A}^{2}- \\
& \|\overrightarrow{M N}\|_{A}^{2}\|\overrightarrow{C N}\|_{A}^{2}-\left(\langle\overrightarrow{C M}, \overrightarrow{M N}\rangle_{A}\right)^{2}+ \\
& \left(\langle\overrightarrow{C N}, \overrightarrow{M N}\rangle_{A}\right)^{2}
\end{aligned}
$$

Combining the first and the fifth terms in the right hand side of the above with aid of 
Equation (1.5) and Definitions 5 and 6 yields

$$
\begin{aligned}
\left(\langle\overrightarrow{C M}, \overrightarrow{M N}\rangle_{A}\right)^{2}-\|\overrightarrow{M N}\|_{A}^{2}\|\overrightarrow{C M}\|_{A}^{2} & =\langle\overrightarrow{C M}, \overrightarrow{M N}\rangle_{A}\left(\langle\overrightarrow{C N}, \overrightarrow{M N}\rangle_{A}+\langle\overrightarrow{M C}, \overrightarrow{M N}\rangle_{A}\right)- \\
& \|\overrightarrow{M N}\|_{A}^{2}\langle\overrightarrow{C M}, \overrightarrow{C N}\rangle_{A}+\|\overrightarrow{M N}\|_{A}^{2}\|\overrightarrow{C M}\|_{A}^{2}- \\
& \|\overrightarrow{M N}\|_{A}^{2}\|\overrightarrow{C N}\|_{A}^{2}+\left(\langle\overrightarrow{C N}, \overrightarrow{M N}\rangle_{A}\right)^{2} \\
& =\langle\overrightarrow{C M}, \overrightarrow{M N}\rangle_{A}\|\overrightarrow{M N}\|_{A}^{2}- \\
& \|\overrightarrow{M N}\|_{A}^{2}\langle\overrightarrow{C M}, \overrightarrow{C N}\rangle_{A}+\|\overrightarrow{M N}\|_{A}^{2}\|\overrightarrow{C M}\|_{A}^{2}- \\
& \|\overrightarrow{M N}\|_{A}^{2}\|\overrightarrow{C N}\|_{A}^{2}-\left(\langle\overrightarrow{C M}, \overrightarrow{M N}\rangle_{A}\right)^{2}+ \\
& \left(\langle\overrightarrow{C N}, \overrightarrow{M N}\rangle_{A}\right)^{2} .
\end{aligned}
$$

Once again, combining the first two terms in the above using Equation (1.5) and Definitions 5 and 6 gives

$$
\begin{aligned}
\left(\langle\overrightarrow{C M}, \overrightarrow{M N}\rangle_{A}\right)^{2}-\|\overrightarrow{M N}\|_{A}^{2}\|\overrightarrow{C M}\|_{A}^{2} & =\|\overrightarrow{M N}\|_{A}^{2}\left(\langle\overrightarrow{C M}, \overrightarrow{M N}\rangle_{A}+\right. \\
& \left.\langle\overrightarrow{C M}, \overrightarrow{N C}\rangle_{A}\right)+\|\overrightarrow{M N}\|_{A}^{2}\|\overrightarrow{C M}\|_{A}^{2}- \\
& \|\overrightarrow{M N}\|_{A}^{2}\|\overrightarrow{C N}\|_{A}^{2}+\left(\langle\overrightarrow{C N}, \overrightarrow{M N}\rangle_{A}\right)^{2} \\
& =-\|\overrightarrow{M N}\|_{A}^{2}\|\overrightarrow{C M}\|_{A}^{2}+\|\overrightarrow{M N}\|_{A}^{2}\|\overrightarrow{C M}\|_{A}^{2}- \\
& \|\overrightarrow{M N}\|_{A}^{2}\|\overrightarrow{C N}\|_{A}^{2}+\left(\langle\overrightarrow{C N}, \overrightarrow{M N}\rangle_{A}\right)^{2},
\end{aligned}
$$

which simplifies to

$$
\left(\langle\overrightarrow{C M}, \overrightarrow{M N}\rangle_{A}\right)^{2}-\|\overrightarrow{M N}\|_{A}^{2}\|\overrightarrow{C M}\|_{A}^{2}=\left(\langle\overrightarrow{C N}, \overrightarrow{M N}\rangle_{A}\right)^{2}-\|\overrightarrow{M N}\|_{A}^{2}\|\overrightarrow{C N}\|_{A}^{2}
$$

It follows from the preceding equation that

$\left(\langle\overrightarrow{C M}, \overrightarrow{M N}\rangle_{A}\right)^{2}-\|\overrightarrow{M N}\|_{A}^{2}\left(\|\overrightarrow{C M}\|_{A}^{2}-\|\overrightarrow{C D}\|_{A}^{2}\right)=\left(\langle\overrightarrow{C M}, \overrightarrow{M N}\rangle_{A}\right)^{2}-$ $\left.\|\overrightarrow{M N}\|\right|_{A} ^{2}\|\overrightarrow{C M}\|_{A}^{2}+\|\overrightarrow{M N}\|_{A}^{2}\|\overrightarrow{C D}\|_{A}^{2}$

$\left(\langle\overrightarrow{C M}, \overrightarrow{M N}\rangle_{A}\right)^{2}-\|\overrightarrow{M N}\|_{A}^{2}\left(\|\overrightarrow{C M}\|_{A}^{2}-\|\overrightarrow{C D}\|_{A}^{2}\right)=\left(\langle\overrightarrow{C N}, \overrightarrow{M N}\rangle_{A}\right)^{2}-$ $\|\overrightarrow{M N}\|_{A}^{2}\|\overrightarrow{C N}\|_{A}^{2}+\|\overrightarrow{M N}\|_{A}^{2}\|\overrightarrow{C D}\|_{A}^{2}$ 


$$
\begin{aligned}
& \quad\left(\langle\overrightarrow{C M}, \overrightarrow{M N}\rangle_{A}\right)^{2}-\|\overrightarrow{M N}\|_{A}^{2}\left(\|\overrightarrow{C M}\|_{A}^{2}-\|\overrightarrow{C D}\|_{A}^{2}\right)=\left(\langle\overrightarrow{C N}, \overrightarrow{M N}\rangle_{A}\right)^{2}+ \\
& \|\overrightarrow{M N}\|_{A}^{2}\left(|| \overrightarrow{C D}\left\|_{A}^{2}-\right\| \overrightarrow{C N} \|_{A}^{2}\right) \mid .
\end{aligned}
$$

Since $\|\overrightarrow{C D}\|_{A}^{2}-\|\overrightarrow{C N}\|_{A}^{2}>0$ by Equation (3.93) and since the other two terms in the right hand side of the preceding equation are positive, we conclude that the Equation (3.92) holds. This ends the proof of this Lemma.

Now, we are ready to prove the Line Circle Intersection Property in arrow spaces.

Theorem 3.12. Let $\mathscr{C}$ be a circle with a center $C$ and a radius $r$, Let $l$ be a line such that $N, M$ are two distinct points on $l$ with $\|\overrightarrow{C N}\|_{A}<r$ and $\|\overrightarrow{C M}\|_{A}>r$. Then, there exists two and only two points $A$ and $B$ on the line l such that $\|\overrightarrow{C A}\|_{A}=\|\overrightarrow{C B}\|_{A}=r$, see figure 3.11 .
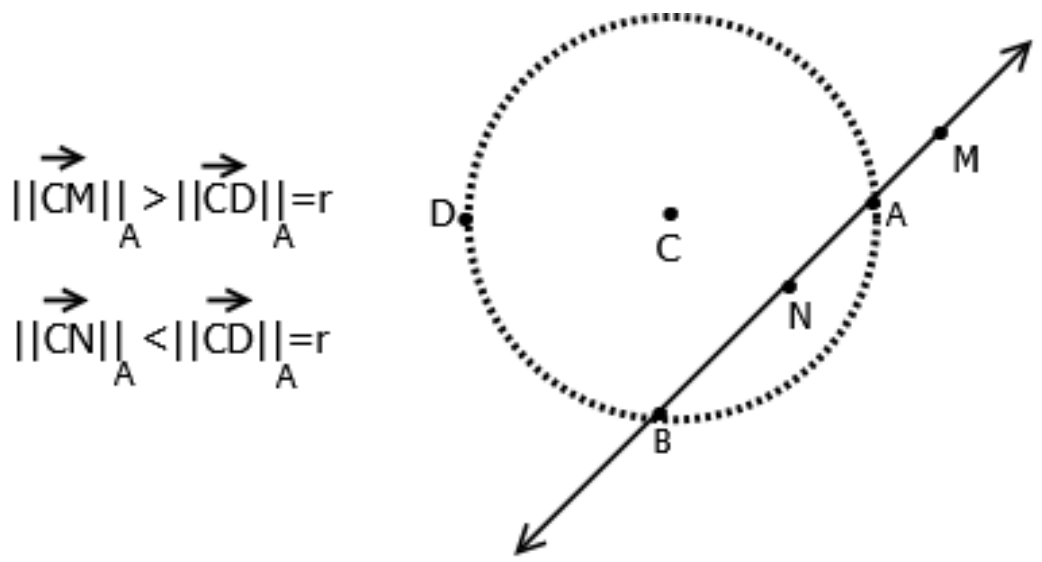

Figure 3.11: Line Circle Intersection Property

Proof : Let $\mathscr{C}$ be a circle with a center $C$ and a radius $r$. Let $D$ be any point on the circle $\mathscr{C}$. This means that $\|\overrightarrow{C D}\|_{A}=r$. Let $l$ be a line that contains two distinct points $N, M$ with

$$
\|\overrightarrow{C N}\|_{A}<\|\overrightarrow{C D}\|_{A},\|\overrightarrow{C M}\|_{A}>\|\overrightarrow{C D}\|_{A}
$$

Now, we are looking for some point (or points) $P$ on $l$ that satisfies the condition $\|\overrightarrow{C P}\|_{A}=r=\|\overrightarrow{C D}\|_{A}$. In other words, $\|\overrightarrow{C P}\|_{A}^{2}=\|\overrightarrow{C D}\|_{A}^{2}$. This means by Definition 6 that $\langle\overrightarrow{C P}, \overrightarrow{C P}\rangle_{A}=\langle\overrightarrow{C D}, \overrightarrow{C D}\rangle_{A}$. This can be rewritten using Definition 5 as follows

$$
\langle\overrightarrow{C P}, \overrightarrow{C P}\rangle_{A}=\|\overrightarrow{C D}\|_{A}^{2}
$$

Notice that, since $N, M$ are on the line $l$ it follows by Theorem 1.11 and Definition 8 that there exists some real number $t_{P}$ such that

$$
\overrightarrow{M P}=t_{P} \overrightarrow{M N}
$$


Then, by Definition 5 Equation (3.96) becomes

$$
\langle\overrightarrow{C P}, \overrightarrow{C P}\rangle_{A}=\langle\overrightarrow{C M}+\overrightarrow{M P}, \overrightarrow{C M}+\overrightarrow{M P}\rangle_{A}=\|\overrightarrow{C D}\|_{A}^{2}
$$

Applying Equations (1.5), (1.2), (1.8), and (3.97) to the preceding equation yields

$$
\begin{aligned}
\langle\overrightarrow{C M}+\overrightarrow{M P}, \overrightarrow{C M}+\overrightarrow{M P}\rangle_{A} & =\|\overrightarrow{C D}\|_{A}^{2} \\
\langle\overrightarrow{C M}, \overrightarrow{C M}\rangle_{A}+2\langle\overrightarrow{C M}, \overrightarrow{M P}\rangle_{A}+\langle\overrightarrow{M P}, \overrightarrow{M P}\rangle_{A} & =\|\overrightarrow{C D}\|_{A}^{2} \\
\|\overrightarrow{C M}\|_{A}^{2}+2 t_{P}\langle\overrightarrow{C M}, \overrightarrow{M N}\rangle_{A}+t_{P}^{2}\|\overrightarrow{M N}\|_{A}^{2} & =\|\overrightarrow{C D}\|_{A}^{2}
\end{aligned}
$$

Rearrainging the above gives the quadratic equation

$$
\|\overrightarrow{M N}\|_{A}^{2} t_{P}^{2}+2\langle\overrightarrow{C M}, \overrightarrow{M N}\rangle_{A} t_{P}+\left(\|\overrightarrow{C M}\|_{A}^{2}-\|\overrightarrow{C D}\|_{A}^{2}\right)=0
$$

which has the solution

$$
t_{P}=\frac{-2\langle\overrightarrow{C M}, \overrightarrow{M N}\rangle_{A} \pm \sqrt{\left(2\langle\overrightarrow{C M}, \overrightarrow{M N}\rangle_{A}\right)^{2}-4\|\overrightarrow{M N}\|_{A}^{2}\left(\|\overrightarrow{C M}\|_{A}^{2}-\|\overrightarrow{C D}\|_{A}^{2}\right)}}{2\|\overrightarrow{M N}\|_{A}^{2}}
$$

It follows now by Lemma 3.2 that the two solutions above are real and distinct. This means by our construction in this proof and by Equation (3.97) that there are exactly two distinct points $A$ and $B$ on the line $l$ that lie, at the same time, on the circle $\mathscr{C}$, which ends the proof of this Theorem.

In the following Theorem we emphasize that if two distinct points $A, B$ lie on a circle, then any point on the line $l_{A B}$ that is between these two points must be inside the circle.

Theorem 3.13. If two distinct points lie on a circle, then any point (on the line that contains the two points) between these two points must be inside the circle, see Figure 3.12 .

Figure 3.12: Points Inside a Circle

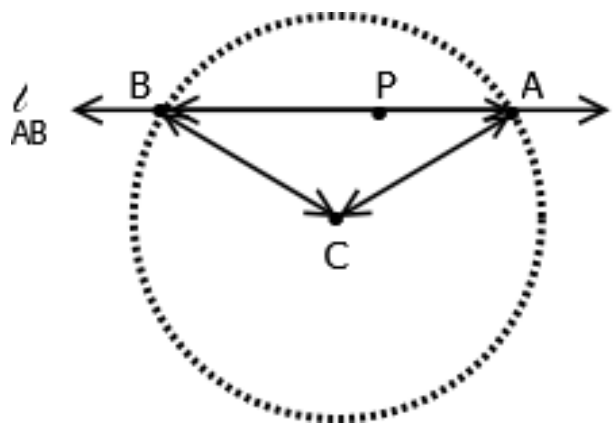


Proof : Let $\mathscr{C}$ be a circle with a center $C$ and a radius $r$. Let $A, B$ be two distinct points with $\|\overrightarrow{C A}\|_{A}=\|\overrightarrow{C B}\|_{A}=r$. Let $l_{A B}$ be the line that contains $A, B$ and $P$ be any point on $l_{A B}$ such that $A \prec P \prec B$. We want to show that the point $P$ is inside the circle $\mathscr{C}$, that is, $\|\overrightarrow{C P}\|_{A}<\|\overrightarrow{C A}\|_{A}$. Now, if $P={ }_{P} C$, then it follows by Proposition 3.12 that $P$ is inside the circle $\mathscr{C}$. Moreover, such point $P$ cannot be on this circle. This is because the line $l_{A B}$ has already two points, $A$ and $B$, that are in common with the circle $\mathscr{C}$ and an application of Theorem 3.12 would role out the possibility of $P$ being on the circle $\mathscr{C}$, that is, $\|\overrightarrow{C P}\|_{A} \neq\|\overrightarrow{C A}\|_{A}$. Now, let $A \prec P \prec B$ with $P \neq_{P} C$. Since $P$ is between $A$ and $B$, it follows by Theorem 3.2 that there exists $t \in(0,1)$ such that

$$
\overrightarrow{A P}=t \overrightarrow{A B}, 0<t<1
$$

If $\|\overrightarrow{C P}\|_{A}<\|\overrightarrow{C A}\|_{A}$, then this is what we want to prove and are done. So assume, by way of contradiction, that

$$
\|\overrightarrow{C A}\|_{A}<\|\overrightarrow{C P}\|_{A}
$$

This means that $\|\overrightarrow{C A}\|_{A}^{2}<\|\overrightarrow{C P}\|_{A}^{2}$. Using Definitions 6, 5, and Equations (1.5), (1.2) we can write the preceding inequality as follows

$$
\begin{aligned}
\|\overrightarrow{C A}\|_{A}^{2} & <\|\overrightarrow{C P}\|_{A}^{2} \\
& <\langle\overrightarrow{C P}, \overrightarrow{C P}\rangle_{A} \\
& <\left\langle\overrightarrow{C A}+_{A} \overrightarrow{A P}, \overrightarrow{C A}+{ }_{A} \overrightarrow{A P}\right\rangle_{A} \\
& <\langle\overrightarrow{C A}, \overrightarrow{C A}\rangle_{A}+2\langle\overrightarrow{C A}, \overrightarrow{A P}\rangle_{A}+\langle\overrightarrow{A P}, \overrightarrow{A P}\rangle_{A} \\
& <\|\overrightarrow{C A}\|_{A}^{2}+2\langle\overrightarrow{C A}, \overrightarrow{A P}\rangle_{A}+\|\overrightarrow{A P}\|_{A}^{2}
\end{aligned}
$$

This simplifies to

$$
\|\overrightarrow{A P}\|_{A}^{2}>-2\langle\overrightarrow{C A}, \overrightarrow{A P}\rangle_{A}
$$

It follows then by Equations (3.98), (1.8), and Lemma 1.9, that the above inequality becomes (notice that $t>0$ and $\|\overrightarrow{A B}\|_{A}^{2} \neq 0$ as $A \neq_{P} B$ )

$$
\begin{aligned}
t^{2}\|\overrightarrow{A B}\|_{A}^{2} & >-2 t\langle\overrightarrow{C A}, \overrightarrow{A B}\rangle_{A} \\
t\|\overrightarrow{A B}\|_{A}^{2} & >-2\langle\overrightarrow{C A}, \overrightarrow{A B}\rangle_{A} \\
t & >\frac{-2\langle\overrightarrow{C A}, \overrightarrow{A B}\rangle_{A}}{\|\overrightarrow{A B}\|_{A}^{2}} .
\end{aligned}
$$


Now, we show that Equation (3.100) is impossible by the following argument, which does not depend on the assumption 3.99, that is, the assumption $\|\overrightarrow{C P}\|_{A}>\|\overrightarrow{C A}\|_{A}$. Indeed, using Definitions 6, 5, and Equations (1.5), (1.2) we have

$$
\begin{aligned}
\|\overrightarrow{C B}\|_{A}^{2} & =\langle\overrightarrow{C B}, \overrightarrow{C B}\rangle_{A} \\
& =\left\langle\overrightarrow{C A}+{ }_{A} \overrightarrow{A B}, \overrightarrow{C A}+{ }_{A} \overrightarrow{A B}\right\rangle_{A} \\
& =\langle\overrightarrow{C A}, \overrightarrow{C A}\rangle_{A}+2\langle\overrightarrow{C A}, \overrightarrow{A B}\rangle_{A}+\langle\overrightarrow{A B}, \overrightarrow{A B}\rangle_{A} \\
& =\|\overrightarrow{C A}\|_{A}^{2}+2\langle\overrightarrow{C A}, \overrightarrow{A B}\rangle_{A}+\|\overrightarrow{A B}\|_{A}^{2}
\end{aligned}
$$

Since $\|\overrightarrow{A B}\|_{A}^{2} \neq 0$ as $A \neq_{P} B$ and by the hypothesis $\|\overrightarrow{C B}\|_{A}^{2}=\|\overrightarrow{C A}\|_{A}^{2}$, the preceding equation simplifies to

$$
\frac{-2\langle\overrightarrow{C A}, \overrightarrow{A B}\rangle_{A}}{\|\overrightarrow{A B}\|_{A}^{2}}=1 .
$$

The above equation is true and it shows that Equation (3.100) means $t>1$. This contradicts Equation (3.98). Therefore, Equation (3.99) is false and we must have $\|\overrightarrow{C P}\|_{A}<\|\overrightarrow{C A}\|_{A}$, as desired.

Theorem 3.14. Let $\mathscr{C}$ be a circle with a center $C$ and a radius $r$. Let $A, B$ be two distinct points with

$$
\|\overrightarrow{C A}\|_{A}=\|\overrightarrow{C B}\|_{A}=r .
$$

Let $l_{A B}$ be the line that contains $A, B$. If $P$ is any point on $l_{A B}$ such that $P$ is inside the circle $\mathscr{C}$, then $P$ must be between $B$ and $A$, that is, $B \prec P \prec A$.

Proof : Let $A, B$ be two distinct points on a circle $\mathscr{C}$ whose center and radius are $C$ and $r$, respectively. Let $P$ be any point on the line $l_{A B}$ such that $P$ is inside $\mathscr{C}$, that is,

$$
\|\overrightarrow{C P}\|_{A}<\|\overrightarrow{C B}\|_{A}=\|\overrightarrow{C A}\|_{A}=r
$$

It follows from the preceding equation that $P \neq_{P} A, P \neq_{P} B$, as otherwise we would have $\|\overrightarrow{C P}\|_{A}=\|\overrightarrow{C B}\|_{A}$ or $\|\overrightarrow{C P}\|_{A}=\|\overrightarrow{C A}\|_{A}$ which contradicts Equation (3.103). Since $P$ is on the line $l_{A B}, A, B$, and it is different than $A$ and $B$ as we just showed, then there exists $t \neq 0$ such that

$$
\overrightarrow{B P}={ }_{A}(t) \overrightarrow{B A}
$$


Notice that, the inequality in 3.103 implies that $\|\overrightarrow{C P}\|_{A}^{2}<\|\overrightarrow{C B}\|_{A}^{2}$ which can be rewritten, using Definitions 6, 5, and Equations (1.5), 1.2 as follows

$$
\begin{aligned}
\|\overrightarrow{C P}\|_{A}^{2} & <\|\overrightarrow{C B}\|_{A}^{2} \\
& <\langle\overrightarrow{C B}, \overrightarrow{C B}\rangle_{A} \\
& <\left\langle\overrightarrow{C P}+{ }_{A} \overrightarrow{P B}, \overrightarrow{C P}+{ }_{A} \overrightarrow{P B}\right\rangle_{A} \\
& <\langle\overrightarrow{C P}, \overrightarrow{C P}\rangle_{A}+2\langle\overrightarrow{C P}, \overrightarrow{P B}\rangle_{A}+\langle\overrightarrow{P B}, \overrightarrow{P B}\rangle_{A} \\
& <\|\overrightarrow{C P}\|_{A}^{2}+2\langle\overrightarrow{C P}, \overrightarrow{P B}\rangle_{A}+\|\overrightarrow{P B}\|_{A}^{2} .
\end{aligned}
$$

The term $\|\overrightarrow{C P}\|_{A}^{2}$ cancels out from both sides of the above inequality, which simplifies after using Definition 4, Equation (1.2), and rearranging to

$$
\begin{gathered}
-2\langle\overrightarrow{C P}, \overrightarrow{P B}\rangle_{A}<\|\overrightarrow{P B}\|_{A}^{2} \\
2\langle\overrightarrow{C P}, \overrightarrow{B P}\rangle_{A}<\|\overrightarrow{P B}\|_{A}^{2} .
\end{gathered}
$$

An application of Definitions 6, 5, and Equations (1.5), (1.2) to the above yields

$$
\begin{aligned}
\|\overrightarrow{P B}\|_{A}^{2} & >2\langle\overrightarrow{C P}, \overrightarrow{B P}\rangle_{A} \\
& >2\left(\langle\overrightarrow{C B}, \overrightarrow{B P}\rangle_{A}+\langle\overrightarrow{B P}, \overrightarrow{B P}\rangle_{A}\right) \\
& >2\langle\overrightarrow{C B}, \overrightarrow{B P}\rangle_{A}+2\|\overrightarrow{B P}\|_{A}^{2}
\end{aligned}
$$

This simplifies by Equation (1.10) to $-\|\overrightarrow{P B}\|_{A}^{2}>2\langle\overrightarrow{C B}, \overrightarrow{B P}\rangle_{A}$, or equivalently

$$
\|\overrightarrow{P B}\|_{A}^{2}<-2\langle\overrightarrow{C B}, \overrightarrow{B P}\rangle_{A}
$$

It follows by Equations (3.104) and (1.8) that the preceding inequality can be rewritten as

$$
\|\overrightarrow{P B}\|_{A}^{2}<-2 t\langle\overrightarrow{C B}, \overrightarrow{B A}\rangle_{A}
$$

Now, we have by Equation (3.101) from Theorem 3.13 that $-2\langle\overrightarrow{C B}, \overrightarrow{B A}\rangle_{A}=\|\overrightarrow{A B}\|_{A}^{2}$ which means that the inequality in (3.106) simplifies to

$$
\|\overrightarrow{P B}\|_{A}^{2}<t\|\overrightarrow{A B}\|_{A}^{2} .
$$


Since $A \neq_{P} B$, we have by Lemma 1.1 that $\|\overrightarrow{A B}\|_{A}^{2} \neq 0$. Thus, dividing both sides of the preceding inequality by $\|\overrightarrow{A B}\|_{A}^{2}$ gives

$$
\frac{\|\overrightarrow{P B}\|_{A}^{2}}{\|\overrightarrow{A B}\|_{A}^{2}}<t
$$

which implies that

$$
t>0 \text {. }
$$

Moreover, we have by 3.104 that $\overrightarrow{B P}=t \overrightarrow{B A}$. Hence, by Equations (1.9) and (1.10) we have

$$
\|\overrightarrow{P B}\|_{A}^{2}=\|(t) \overrightarrow{A B}\|_{A}^{2}=t^{2}\|\overrightarrow{B A}\|_{A}^{2}=t^{2}\|\overrightarrow{A B}\|_{A}^{2}
$$

If we plug in the above equation to the inequality in 3.108 , we get

$$
\frac{t^{2}\|\overrightarrow{A B}\|_{A}^{2}}{\|\overrightarrow{A B}\|_{A}^{2}}<t
$$

that is, $t^{2}<t$. Since we have by 3.109 that $t>0$, then if we divide both sides in the preceding inequality by $t$, we get

$$
t<1 \text {. }
$$

Combining Equations (3.109), (3.111), and (3.104) we conclude that there exists $t \in(0,1)$ such that $\overrightarrow{B P}=t \overrightarrow{B A}$. This means by Theorem 3.1 that $P$ is between $B$ and $A$, as desired.

Next, we introduce a theorem that will lead to the recognition of the tangent line of a circle.

Theorem 3.15. Let $\mathscr{C}$ be a circle with a center $C$ and a radius $r$. Let $A$ be any point on $\mathscr{C}$, that is, $\|\overrightarrow{C A}\|_{A}=r$. If $B$ is any point different than $A$ such that $\langle\overrightarrow{C A}, \overrightarrow{A B}\rangle_{A}=0$, then for any $P \in l_{A B}$ with $P \neq_{P} A$ we must have $\|\overrightarrow{C P}\|_{A}>\|\overrightarrow{C A}\|_{A}$, that is, $P$ is outside the circle $\mathscr{C}$, see Figure 3.13 . 


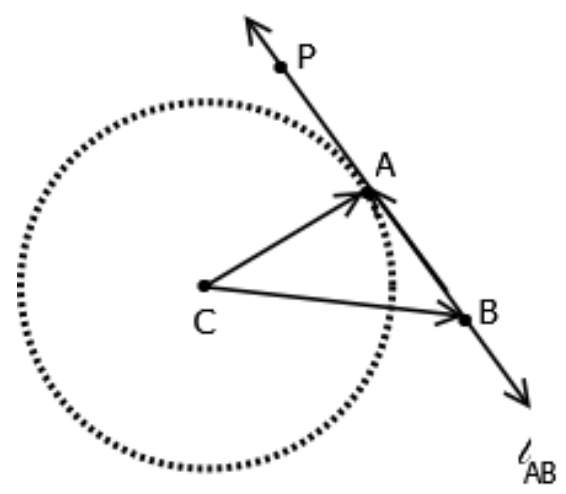

Figure 3.13: An illustration of Theorem 3.15.

Proof : Let $A$ be a point on a circle $\mathscr{C}$ with a center $C$ and a radius $r$. Let $B \neq_{P} A$ be any point such that

$$
\langle\overrightarrow{C A}, \overrightarrow{A B}\rangle_{A}=0
$$

We know, by means of Definition 8 , that any point $P$, other than $A$, on the line $l_{A B}$ is given by the equation

$$
\overrightarrow{A P}={ }_{A} t \overrightarrow{A B}, t \neq 0
$$

Thus, we have by Equations (3.112), (1.8), and (3.113) that

$$
\langle\overrightarrow{C A}, \overrightarrow{A P}\rangle_{A}=\langle\overrightarrow{C A}, t \overrightarrow{A B}\rangle_{A}=t\langle\overrightarrow{C A}, \overrightarrow{A B}\rangle_{A}=0
$$

Now, using Definitions 6, 5, Equations (1.5), (1.2), and (3.114) we have

$$
\begin{aligned}
0=\langle\overrightarrow{C A}, \overrightarrow{A P}\rangle_{A} & =\left\langle\overrightarrow{C P}+_{A} \overrightarrow{P A}, \overrightarrow{A P}\right\rangle_{A}=\langle\overrightarrow{C P}, \overrightarrow{A P}\rangle_{A}+\langle\overrightarrow{P A}, \overrightarrow{A P}\rangle_{A} \\
& =\langle\overrightarrow{C P}, \overrightarrow{A P}\rangle_{A}-\|\overrightarrow{A P}\|_{A}^{2}
\end{aligned}
$$

which means, by Definition 6 and Equation (1.6), that

$$
\langle\overrightarrow{C P}, \overrightarrow{P A}\rangle_{A}=-\|\overrightarrow{A P}\|_{A}^{2}
$$

On the other hand, using Definitions 6, 5, Equations (1.5), (1.2), and (3.115) we have

$$
\begin{aligned}
\|\overrightarrow{C A}\|_{A}^{2} & =\langle\overrightarrow{C A}, \overrightarrow{C A}\rangle_{A}=\left\langle\overrightarrow{C P}+{ }_{A} \overrightarrow{P A}, \overrightarrow{C P}+{ }_{A} \overrightarrow{P A}\right\rangle_{A} \\
& =\|\overrightarrow{C P}\|_{A}^{2}+2\langle\overrightarrow{C P}, \overrightarrow{P A}\rangle_{A}+\|\overrightarrow{A P}\|_{A}^{2} \\
& =\|\overrightarrow{C P}\|_{A}^{2}-2\|\overrightarrow{A P}\|_{A}^{2}+\|\overrightarrow{A P}\|_{A}^{2} \\
& =\|\overrightarrow{C P}\|_{A}^{2}-\|\overrightarrow{A P}\|_{A}^{2} .
\end{aligned}
$$


Since $A \neq_{P} P$, we have by Lemma 1.1 that $\|\overrightarrow{A P}\|_{A}^{2} \neq 0$. Hence, the preceding equation implies that

$$
\|\overrightarrow{C A}\|_{A}^{2}=\|\overrightarrow{C P}\|_{A}^{2}-\|\overrightarrow{A P}\|_{A}^{2}<\|\overrightarrow{C P}\|_{A}^{2}
$$

Taking the positive square root of both sides of the above yields $\|\overrightarrow{C A}\|_{A}<\|\overrightarrow{C P}\|_{A}$. The preceding inequality means by Definition 22 that $P$ is outside the circle which ends the proof of this theorem.

Notice that, Theorem 3.15 gives only a description of the "tangent line" and does not guarantee the existence and uniqueness of a tangent line of a circle at some point on this circle. The following Theorem confirms the existence and uniqueness of a "tangent line" at any given point on a circle.

Theorem 3.16. Let $\mathscr{C}$ be a circle with a center $C$ and a radius $r$. Let $A$ be any point on $\mathscr{C}$, that is, $\|\overrightarrow{C A}\|_{A}=r$. Then, there exists a unique line l that passes through $A$ such that

$$
\langle\overrightarrow{C A}, \overrightarrow{A B}\rangle_{A}=0
$$

for any point B on l, see Figure 3.14.

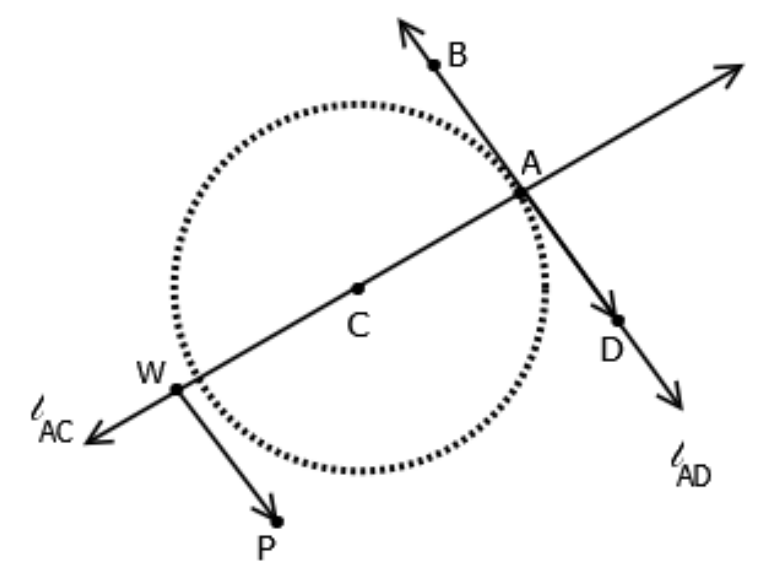

Figure 3.14: The Existence of a Tangent Line of a Circle

Proof : Let $\mathscr{C}$ be a circle with a center $C$ and a radius $r$ and let $A$ be any point such that $\|\overrightarrow{C A}\|_{A}=r$. Since $r>0$, we see that $C \neq_{P} A$ as otherwise we would have $\|\overrightarrow{C A}\|_{A}=r=0$. Then, we have by Theorem 1.11 that there exists a unique line $l_{A C}$ that contains $A$ and $C$. Now, let $P$ be any point that does not lie on the line $l_{A C}$. Then, an application of Theorem 2.12 to the line $l_{A C}$ and the point $P$ implies that there exists a unique point $W$ on $l_{A C}$ such that

$$
\langle\overrightarrow{W C}, \overrightarrow{W P}\rangle_{A}=0
$$


For the point $W$, we consider the following three possible cases:

.1 If $W={ }_{P} A$, then Equation (3.117) would become $\langle\overrightarrow{A C}, \overrightarrow{A P}\rangle_{A}=0$, which is equivalent by Definition 4 and Equation (1.6) to the equation $\langle\overrightarrow{C A}, \overrightarrow{A P}\rangle_{A}=0$ Thus, by means of Theorem $1.11, l_{A P}$ is the unique line that passes through $A, P$. Moreover, it follows by Corollary 2.2 that any point $B$ on $l_{A P}$ satisfies Equation (3.116), that is, $\langle\overrightarrow{C A}, \overrightarrow{A B}\rangle_{A}=0$.

2. If $W \neq{ }_{P} A$ and $W={ }_{P} C$, then since the proof of Theorem 2.12 does not depend on the choice of any of the points $A$ and $C$ we would also have

$$
\langle\overrightarrow{C A}, \overrightarrow{C P}\rangle_{A}=0
$$

Now, if we apply Equation (2.7) to the arrow $\overrightarrow{C P}$ and the point $A$, we get a unique arrow $\overrightarrow{A D}$ such that

$$
\overrightarrow{C P} \Re \overrightarrow{A D}
$$

Since the relation $\Re$ is reflexive, we also have $\overrightarrow{C A} \Re \overrightarrow{C A}$. If we apply Equation (2.39) to the preceding relation and the relation in 3.119, we get that

$$
\langle\overrightarrow{C A}, \overrightarrow{C P}\rangle_{A}=\langle\overrightarrow{C A}, \overrightarrow{A D}\rangle_{A}
$$

But by Equation (3.118) we have $\langle\overrightarrow{C A}, \overrightarrow{C P}\rangle_{A}=0$. This means by Equation (3.120) that $\langle\overrightarrow{C A}, \overrightarrow{A D}\rangle_{A}=0$. Notice that, we have by Theorem 1.11 that $l_{A D}$ is the unique line that passes through $A, D$. Moreover, it follows by Corollary 2.2 that any point $B$ on $l_{A D}$ satisfies Equation (3.116), that is $\langle\overrightarrow{C A}, \overrightarrow{A B}\rangle_{A}=0$, which ends the proof for this case.

3. If $W \neq_{P} A$ and $W \neq_{P} C$, then since $A, C$, and $W$ are points on the line $l_{A C}$ we conclude from Equation (3.117) and Corollary 2.2 that

$$
\langle\overrightarrow{C A}, \overrightarrow{W P}\rangle_{A}=0 \text {. }
$$

If we apply Equation (2.7) to the arrow $\overrightarrow{W P}$ and the point $A$, we get a unique arrow $\overrightarrow{A D}$ such that

$$
\overrightarrow{W P} \Re \overrightarrow{A D}
$$

Since the relation $\Re$ is reflexive we also have $\overrightarrow{C A} \Re \overrightarrow{C A}$. If we apply Equation (2.39) to the preceding relation and the relation in 3.122, we get that

$$
\langle\overrightarrow{C A}, \overrightarrow{W P}\rangle_{A}=\langle\overrightarrow{C A}, \overrightarrow{A D}\rangle_{A}
$$


But by Equation (3.121) we have $\langle\overrightarrow{C A}, \overrightarrow{W P}\rangle_{A}=0$. This means by Equation (3.123) that $\langle\overrightarrow{C A}, \overrightarrow{A D}\rangle_{A}=0$. Notice that, we have by Theorem 1.11 that $l_{A D}$ is the unique line that passes through $A, D$. Moreover, it follows by Corollary 2.2 that any point $B$ on $l_{A D}$ satisfies Equation (3.116), that is $\langle\overrightarrow{C A}, \overrightarrow{A B}\rangle_{A}=0$, which ends the proof for this theorem.

Now, we can define a tangent line of a circle at a point.

Definition 23. Let $\mathscr{C}$ be a circle with a center $C$. Let $A$ be any point on $\mathscr{C}$. Then, we define the unique line $l$ (which is obtained from Theorem 3.16) that passes through $A$ such that $\langle\overrightarrow{C A}, \overrightarrow{A B}\rangle_{A}=0$ for any point $B$ on $l$ the tangent line of $\mathscr{C}$ at $A$. See Figure 3.15 .

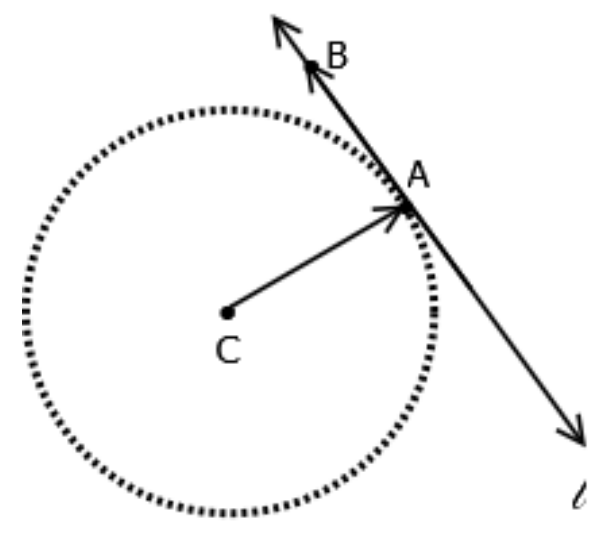

Figure 3.15: A Tangent Line of a Circle in an Arrow Space.

\subsection{Triangles in Arrow Spaces}

In this section, we study more geometrical properties in an arrow space. These properties are related to similar, congruent, and right triangles, as well as the Pythagorean Theorem. Using our tools of an arrow space, we prove the well known Side-AngleSide criterion for congruence of triangles. In his book, Euclid proves this criterion using superposition, a method that has been criticized as it cannot be justified on the basis of his axioms. Hilbert, on the other hand, imposes it as an axiom. In [20], Hartshorne shows that in a geometry over an ordered field, there exist enough rigid motions (mappings that preserve the structure in such geometry) to prove the SideAngle-Side criterion. In this dissertation, we utilize arrow space tools in order to bring this about as a theorem (theorem 3.19). Since this theorem involves triangles, and we have not yet defined a triangle, we start by defining a triangle in arrow spaces. 
Definition 24. Let $A, B$, and $C$ be three points such that $C \notin l_{A B}$. We call $A, B$, and $C$ noncollinear points and define a triangle that is formed by $A, B$, and $C$, denoted by $\triangle A B C$, to be the set of the points $A, B$, and $C$ together with all points $P$, where $A \prec P \prec B, A \prec P \prec C$, and $B \prec P \prec C$, see Figure 3.16.

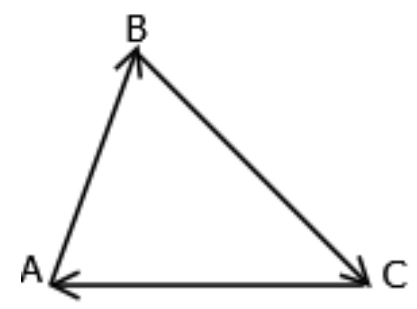

Figure 3.16: A Triangle

Now that we defined a triangle, we would like now to implement some results from Sections 2.1 and 2.4 to appoint some special types of triangles. We start with a definition of the right triangle which exists as a result of Theorem 2.12.

Definition 25. If the pre-inner product of two of the three arrows that form a triangle $\triangle A B C$ is zero, say $\langle\overrightarrow{A B}, \overrightarrow{A C}\rangle_{A}=0$, then this triangle is called a right triangle, see Figure 3.17.

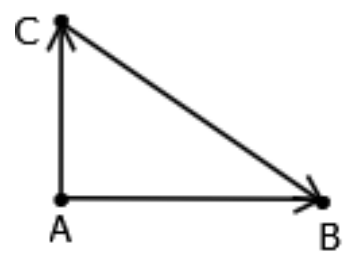

Figure 3.17: A Right Triangle.

Remark 3.4. Notice that, according to Theorem 2.1 and the preceding definition, we see that Pythagorean Theorem holds in arrow spaces.

We presented in Proposition 2.1 a triangle that is formed by three arrows two of which had equal length. This triangle is called an isosceles triangle.

Definition 26. If two of the three arrows that form a triangle $\triangle A B C$ have equal length, say $\|\overrightarrow{A B}\|_{A}=\|\overrightarrow{A C}\|_{A}$, the $\triangle A B C$ is called an isosceles triangle. 
With the aid of Definition 18, we show that the Law of Cosines holds in any arrow space $\mathscr{P}_{A}$.

Theorem 3.17. (The Law of Cosine for Arrow Spaces) Let A, B, and $C$ be three points such that $\overrightarrow{A B}, \overrightarrow{A C}$ and $\overrightarrow{B C}$ form a Triangle $\triangle A B C$, as illustrated in Figure 3.18. Then,

$$
\left\|\overrightarrow{A B}+{ }_{A} \overrightarrow{B C}\right\|_{A}^{2}=\|\overrightarrow{A B}\|_{A}^{2}+\|\overrightarrow{B C}\|_{A}^{2}-2\|\overrightarrow{A B}\|_{A}\|\overrightarrow{C B}\|_{A} \cos \angle B A C
$$

if and only if $\cos \angle B A C=\frac{\langle\overrightarrow{B A}, \overrightarrow{B C}\rangle_{A}}{\|\overrightarrow{B A}\|_{A}\|\overrightarrow{B C}\|_{A}}$.

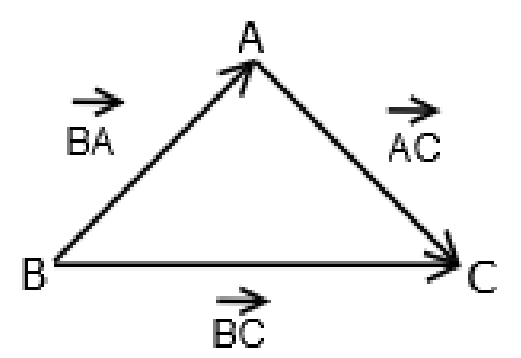

Figure 3.18: A Triangle for The Law of Cosines.

Proof : Notice that, by Equations (1.6), (1.5), and Definition 6 we can write

$$
\begin{aligned}
\left\|\overrightarrow{A B}+{ }_{A} \overrightarrow{B C}\right\|_{A}^{2} & =\left\langle\overrightarrow{A B}+{ }_{A} \overrightarrow{B C}, \overrightarrow{A B}+{ }_{A} \overrightarrow{B C}\right\rangle_{A} \\
& =\|\overrightarrow{A B}\|_{A}^{2}+\|\overrightarrow{B C}\|_{A}^{2}+2\langle\overrightarrow{A B}, \overrightarrow{B C}\rangle_{A} \\
& =\|\overrightarrow{A B}\|_{A}^{2}+\|\overrightarrow{B C}\|_{A}^{2}-2\langle\overrightarrow{B A}, \overrightarrow{B C}\rangle_{A}
\end{aligned}
$$

Rearrainging the preceding equation by multiplying the third term in the right hand side by (notice that $\|\overrightarrow{B A}\|_{A},\|\overrightarrow{B C}\|_{A} \neq 0$ as $A \neq \neq_{P} B, B \neq{ }_{P} C$ )

$$
\frac{\|\overrightarrow{B A}\|_{A}\|\overrightarrow{B C}\|_{A}}{\|\overrightarrow{B A}\|_{A}\|\overrightarrow{B C}\|_{A}}
$$

yields

$$
\left\|\overrightarrow{A B}+{ }_{A} \overrightarrow{B C}\right\|_{A}^{2}=\|\overrightarrow{A B}\|_{A}^{2}+\|\overrightarrow{B C}\|_{A}^{2}-\|\overrightarrow{B A}\|_{A}\|\overrightarrow{B C}\|_{A} \frac{\langle\overrightarrow{B A}, \overrightarrow{B C}\rangle_{A}}{\|\overrightarrow{B A}\|_{A}\|\overrightarrow{B C}\|_{A}}
$$


If Definition 18 holds, Equation (3.125) becomes Equation (3.124). On the other hand, applying the Laws of Cosines from classical trigonometry to the triangle of Figure 3.18 gives us Equation (3.124). Therefore, if we compare Equation (3.124) with Equation (3.125), we deduce that

$$
\cos \angle B A C=\frac{\langle\overrightarrow{B A}, \overrightarrow{B C}\rangle_{A}}{\|\overrightarrow{B A}\|_{A}\|\overrightarrow{B C}\|_{A}} .
$$

Definition 27. Let $A, B$, and $C$ be three distinct points. Let $\angle B A C$ be the angle between $\overrightarrow{B A}_{\infty}$ and $\overrightarrow{B C}_{\infty}$. Define a sine function of $\angle B A C$ as

$$
\sin \angle B A C=\sqrt{1-\cos ^{2} \angle B A C} .
$$

Proposition 3.7 shows that $0 \leq \sin \angle B A C \leq 1$.

We next show that the Law of Sines holds for arrow spaces.

Theorem 3.18. (The Law of Sines for an arrow space $\mathscr{P}_{A}$ ) Let $A, B$, and $C$ be three points in an arrow space $\mathscr{P}_{A}$ such that $\overrightarrow{A B}, \overrightarrow{A C}$ and $\overrightarrow{B C}$ form a Triangle $\triangle A B C$, as illustrated in Figure 3.19. Then

$$
\frac{\sin \angle C A B}{\|\overrightarrow{C B}\|_{A}}=\frac{\sin \angle B C A}{\|\overrightarrow{A B}\|_{A}}=\frac{\sin \angle A B C}{\|\overrightarrow{A C}\|_{A}}=q(\overrightarrow{A C}, \overrightarrow{A B}),
$$

where

$$
q^{2}(\overrightarrow{A C}, \overrightarrow{A B})=\frac{\|\overrightarrow{A C}\|_{A}^{2}\|\overrightarrow{A B}\|_{A}^{2}-\left(\langle\overrightarrow{A B}, \overrightarrow{A C}\rangle_{A}\right)^{2}}{\|\overrightarrow{A C}\|_{A}^{2}\|\overrightarrow{A B}\|_{A}^{2}\|\overrightarrow{B C}\|_{A}^{2}} .
$$

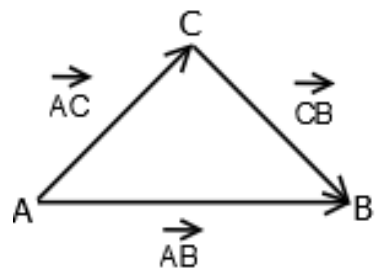

Figure 3.19: A Triangle for The Law of Sines.

Proof : Let $\cos \angle C A B=\frac{\langle\overrightarrow{A C}, \overrightarrow{A B}\rangle_{A}}{2\|\overrightarrow{A C}\|_{A}\|\overrightarrow{A B}\|_{A}}$. We want to show that Equation (3.127) holds. We will show that

$$
\frac{\sin \angle C A B}{\|\overrightarrow{C B}\|_{A}}=\frac{\sin \angle B C A}{\|\overrightarrow{A B}\|_{A}}
$$


and leave the details of proving the similar identity

$$
\frac{\sin \angle A B C}{\|\overrightarrow{A C}\|_{A}}=\frac{\sin \angle B C A}{\|\overrightarrow{A B}\|_{A}}
$$

to the reader. To prove Equation (3.128), it is enough to show that

$$
\frac{\sin ^{2} \angle C A B}{\|\overrightarrow{C B}\|_{A}^{2}}=\frac{\sin ^{2} \angle B C A}{\|\overrightarrow{A B}\|_{A}^{2}}
$$

since Definition 27 implies that the sine function has nonnegative range. Furthermore, Definition 27 implies that Equation (3.128) is equivalent to

$$
\frac{1-\cos ^{2} \angle C A B}{\|\overrightarrow{C B}\|_{A}^{2}}=\frac{1-\cos ^{2} \angle B C A}{\|\overrightarrow{A B}\|_{A}^{2}} .
$$

Thus, in order to show Equation (3.128) we need only to show that

$$
\|\overrightarrow{A B}\|_{A}^{2}\left(1-\cos ^{2} \angle C A B\right)=\|\overrightarrow{C B}\|_{A}^{2}\left(1-\cos ^{2} \angle B C A\right) .
$$

Notice that, by Definition 5, 6, Equations (1.5), (1.6), and (1.2) we can write

$$
\begin{aligned}
\|\overrightarrow{A B}\|_{A}^{2} & =\langle\overrightarrow{A B}, \overrightarrow{A B}\rangle_{A}=\left\langle\overrightarrow{A C}+{ }_{A} \overrightarrow{C B}, \overrightarrow{A C}+{ }_{A} \overrightarrow{C B}\right\rangle_{A} \\
& =\|\overrightarrow{A C}\|_{A}^{2}+\|\overrightarrow{C B}\|_{A}^{2}+2\langle\overrightarrow{A C}, \overrightarrow{C B}\rangle_{A} \\
& =\|\overrightarrow{A C}\|_{A}^{2}+\|\overrightarrow{C B}\|_{A}^{2}-2\langle\overrightarrow{C A}, \overrightarrow{A B}\rangle_{A} .
\end{aligned}
$$

Also, following a similar calculation to the above we can write

$$
\begin{aligned}
\cos ^{2} \angle C A B & =\frac{\left(\langle\overrightarrow{A B}, \overrightarrow{A C}\rangle_{A}\right)^{2}}{\|\overrightarrow{A B}\|_{A}^{2}\|\overrightarrow{A C}\|_{A}^{2}}=\frac{\left(\left\langle\overrightarrow{A C}+{ }_{A} \overrightarrow{C B}, \overrightarrow{A C}\right\rangle_{A}\right)^{2}}{\|\overrightarrow{A B}\|_{A}^{2}\|\overrightarrow{A C}\|_{A}^{2}} \\
& =\frac{\left(\|\overrightarrow{A C}\|_{A}^{2}-\langle\overrightarrow{C A}, \overrightarrow{C B}\rangle_{A}\right)^{2}}{\|\overrightarrow{A B}\|_{A}^{2}\|\overrightarrow{A C}\|_{A}^{2}} .
\end{aligned}
$$

Now, we rewrite the left hand side of Equation 3.129 using the preceding equation and Equation (3.130) as follows

$$
\begin{aligned}
\|\overrightarrow{A B}\|_{A}^{2}\left(1-\cos ^{2} \angle C A B\right) & =\|\overrightarrow{A B}\|_{A}^{2}-\|\overrightarrow{A B}\|_{A}^{2} \frac{\left(\|\overrightarrow{A C}\|_{A}^{2}-\langle\overrightarrow{C A}, \overrightarrow{C B}\rangle_{A}\right)^{2}}{\|\overrightarrow{A B}\|_{A}^{2}\|\overrightarrow{A C}\|_{A}^{2}} \\
& =\frac{\|\overrightarrow{A B}\|_{A}^{2}\|\overrightarrow{A C}\|_{A}^{2}-\left[\|\overrightarrow{A C}\|_{A}^{2}-\langle\overrightarrow{C A}, \overrightarrow{C B}\rangle_{A}\right]^{2}}{\|\overrightarrow{A C}\|_{A}^{2}} .
\end{aligned}
$$


The numerator of the right hand side of the above simplifies nicely. Indeed, if we, use Equation (3.130) to substitute for $\|\overrightarrow{A B}\|_{A}^{2}$, expand the second term, and simplify, we get

$$
\begin{aligned}
\|\overrightarrow{A B}\|_{A}^{2}\left(1-\cos ^{2} \angle C A B\right) & =\frac{\|\overrightarrow{A C}\|_{A}^{2}\|\overrightarrow{C B}\|_{A}^{2}-\left(\langle\overrightarrow{C A}, \overrightarrow{C B}\rangle_{A}\right)^{2}}{\|\overrightarrow{A C}\|_{A}^{2}} \\
& =\|\overrightarrow{C B}\|_{A}^{2}-\frac{\left(\langle\overrightarrow{C A}, \overrightarrow{C B}\rangle_{A}\right)^{2}}{\|\overrightarrow{A C}\|_{A}^{2}} \\
& =\|\overrightarrow{C B}\|_{A}^{2}\left(1-\frac{\left(\langle\overrightarrow{C A}, \overrightarrow{C B}\rangle_{A}\right)^{2}}{\|\overrightarrow{C B}\|_{A}^{2}\|\overrightarrow{C A}\|_{A}^{2}}\right)
\end{aligned}
$$

By means of Definition 18, the above is equivalent to

$$
\|\overrightarrow{A B}\|_{A}^{2}\left(1-\cos ^{2} \angle C A B\right)=\|\overrightarrow{C B}\|_{A}^{2}\left(1-\cos ^{2} \angle B C A\right),
$$

which is precisely the identity of Equation (3.129).

Observe that when verifying Equation (3.129), we showed that

$$
\|\overrightarrow{A B}\|_{A}^{2} \sin ^{2} \angle C A B=\frac{\|\overrightarrow{A B}\|_{A}^{2}\|\overrightarrow{A C}\|_{A}^{2}-\left[\langle\overrightarrow{A B}, \overrightarrow{A C}\rangle_{A}\right]^{2}}{\|\overrightarrow{A C}\|_{A}^{2}}
$$

This in turn leads to the auxiliary identity

$$
\frac{\sin ^{2} \angle C A B}{\|\overrightarrow{C B}\|_{A}}=\frac{1-\cos ^{2} \angle C A B}{\|\overrightarrow{C B}\|_{A}}=\frac{\|\overrightarrow{A B}\|_{A}^{2}\|\overrightarrow{A C}\|_{A}^{2}-\left[\langle\overrightarrow{A B}, \overrightarrow{A C}\rangle_{A}\right]^{2}}{\|\overrightarrow{A C}\|_{A}^{2}\|\overrightarrow{A B}\|_{A}^{2}\|\overrightarrow{C B}\|_{A}^{2}}
$$

Now, assume that Equation (3.127) holds. Then

$$
q^{2}(\overrightarrow{A C}, \overrightarrow{A B})=\frac{\sin ^{2} \angle C A B}{\|\overrightarrow{C B}\|_{A}}=\frac{1-\cos ^{2} C A B}{\|\overrightarrow{C B}\|_{A}}=\frac{\|\overrightarrow{A B}\|_{A}^{2}\|\overrightarrow{A C}\|_{A}^{2}-\left[\langle\overrightarrow{A B}, \overrightarrow{A C}\rangle_{A}\right]^{2}}{\|\overrightarrow{A C}\|_{A}^{2}\|\overrightarrow{A B}\|_{A}^{2}\|\overrightarrow{C B}\|_{A}^{2}}
$$


This means

$$
\begin{aligned}
\sin ^{2} \angle C A B=1-\cos ^{2} \angle C A B & =\frac{\|\overrightarrow{A B}\|_{A}^{2}\|\overrightarrow{A C}\|_{A}^{2}-\left[\langle\overrightarrow{A B}, \overrightarrow{A C}\rangle_{A}\right]^{2}}{\|\overrightarrow{A C}\|_{A}^{2}\|\overrightarrow{A B}\|_{A}^{2}} \\
& =1-\frac{\left[\langle\overrightarrow{A B}, \overrightarrow{A C}\rangle_{A}\right]^{2}}{\|\overrightarrow{A C}\|_{A}^{2}\|\overrightarrow{A B}\|_{A}^{2}}
\end{aligned}
$$

which in turn implies

$$
\cos ^{2} \angle C A B=\frac{\left[\langle\overrightarrow{A B}, \overrightarrow{A C}\rangle_{A}\right]^{2}}{\|\overrightarrow{A B}\|_{A}^{2}\|\overrightarrow{A C}\|_{A}^{2}}
$$

as desired.

\subsection{Similarity of Triangles.}

We start with the definition of similar triangles and then prove the similarity theorems of triangles in arrow spaces.

Definition 28. Let $\triangle A B C, \triangle E F G$ be two triangles in an arrow space $\mathscr{P}_{A}$, their sides determined by the arrows $\overrightarrow{A B}, \overrightarrow{A C}, \overrightarrow{B C}$ and $\overrightarrow{E F}, \overrightarrow{E G}, \overrightarrow{F G}$, respectively, as illustrated in Figure 3.20. We say that Triangle $\triangle A B C$ is similar to Triangle $\triangle E F G$ if the corresponding sides are in proportion. That is, there exists a positive real number $s$ such that $\frac{\|\overrightarrow{A B}\|_{A}}{\|\overrightarrow{E F}\|_{A}}=\frac{\|\overrightarrow{B C}\|_{A}}{\|\overrightarrow{F G}\|_{A}}=\frac{\|\overrightarrow{A C}\|_{A}}{\|\overrightarrow{E G}\|_{A}}=s$. In particular, if $s=1$, we say that the Triangles $\triangle A B C$ and $\triangle E F G$ are congruent.
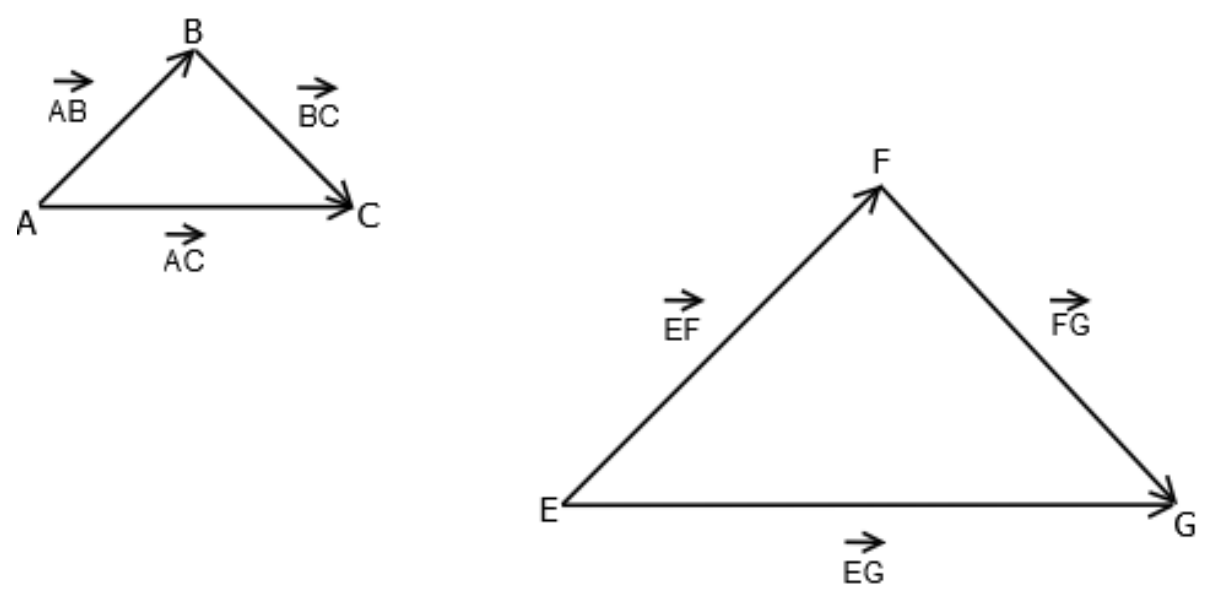

Figure 3.20: Similar Triangles a an arrow space $\mathscr{P}_{A}$. 
Now, we prove the Side-Angle-Side Similarity Theorem from which we conclude that Side-Angle-Side (S.A.S.) congruent Theorem of Triangles hold in arrow spaces, where $s=1$.

Theorem 3.19. (Side-Angle-Side Similarity Theorem in Arrow Spaces) Let $\triangle A B C, \triangle E F G$ be two triangles in an arrow space $\mathscr{P}_{A}$, as in Figure 3.20. If $\cos \angle A B C=\cos \angle E F G$, and $\frac{\|\overrightarrow{A B}\|_{A}}{\|\overrightarrow{E F}\|_{A}}=\frac{\|\overrightarrow{B C}\|_{A}}{\|\overrightarrow{F G}\|_{A}}=s$, then $\triangle E F G$ and $\triangle A B C$ are similar.

Proof : Let $\triangle E F G$ and $\triangle A B C$ be two triangles as illustrated in Figure 4. Since $\cos \angle A B C=\cos \angle E F G$, we have

$$
\frac{\langle\overrightarrow{B A}, \overrightarrow{B C}\rangle_{A}}{\|\overrightarrow{B A}\|_{A}\|\overrightarrow{B C}\|_{A}}=\frac{\langle\overrightarrow{F E}, \overrightarrow{F G}\rangle_{A}}{\|\overrightarrow{F E}\|_{A}\|\overrightarrow{F G}\|_{A}}
$$

Let

$$
\frac{\|\overrightarrow{A B}\|_{A}}{\|\overrightarrow{E F}\|_{A}}=\frac{\|\overrightarrow{B C}\|_{A}}{\|\overrightarrow{F G}\|_{A}}=s, \quad s>0
$$

It is enough to show that

$$
\frac{\|\overrightarrow{A C}\|_{A}}{\|\overrightarrow{E G}\|_{A}}=s
$$

Notice that, by Definitions 5, 6, Equations (1.8), (1.2), and (1.5) we can write

$$
\begin{aligned}
\|\overrightarrow{A C}\|_{A}^{2} & =\langle\overrightarrow{A C}, \overrightarrow{A C}\rangle_{A}=\left\langle\overrightarrow{A B}+{ }_{A} \overrightarrow{B C}, \overrightarrow{A B}+_{A} \overrightarrow{B C}\right\rangle_{A} \\
& =\|\overrightarrow{A B}\|_{A}^{2}+\|\overrightarrow{B C}\|_{A}^{2}+2\langle\overrightarrow{A B}, \overrightarrow{B C}\rangle_{A} \\
& =\|\overrightarrow{A B}\|_{A}^{2}+\|\overrightarrow{B C}\|_{A}^{2}-2\langle\overrightarrow{B A}, \overrightarrow{B C}\rangle_{A} .
\end{aligned}
$$

This is equivalent to

$$
\|\overrightarrow{A C}\|_{A}^{2}=\|\overrightarrow{A B}\|_{A}^{2}+\|\overrightarrow{B C}\|_{A}^{2}-2\|\overrightarrow{A B}\|_{A}\|\overrightarrow{B C}\|_{A} \frac{\langle\overrightarrow{B A}, \overrightarrow{B C}\rangle_{A}}{\|\overrightarrow{A B}\|_{A}\|\overrightarrow{B C}\|_{A}}
$$

Using Equations (3.132) and (3.133), the preceding equation can be rewritten as

$$
\begin{aligned}
\|\overrightarrow{A C}\|_{A}^{2} & =s^{2}\|\overrightarrow{E F}\|_{A}^{2}+s^{2}\|\overrightarrow{F G}\|_{A}^{2}-2 s^{2}\|\overrightarrow{F E}\|_{A}\|\overrightarrow{F G}\|_{A} \frac{\langle\overrightarrow{F E}, \overrightarrow{F G}\rangle_{A}}{\|\overrightarrow{F E}\|\|\overrightarrow{F G}\|_{A}} \\
& =s^{2}\left[\|\overrightarrow{E F}\|_{A}^{2}+\|\overrightarrow{F G}\|_{A}^{2}-2\langle\overrightarrow{F E}, \overrightarrow{F G}\rangle_{A}\right] .
\end{aligned}
$$


Again, by Definitions 5, 6, Equations (1.8), (1.2), and (1.5) we can rewrite the right hand side of the preceding equation as follows

$$
\begin{aligned}
s^{2}\left[\|\overrightarrow{E F}\|_{A}^{2}+\|\overrightarrow{F G}\|_{A}^{2}-2\langle\overrightarrow{F E}, \overrightarrow{F G}\rangle_{A}\right] & =s^{2}\left\langle\overrightarrow{E F}+{ }_{A} \overrightarrow{F G}, \overrightarrow{E F}+_{A} \overrightarrow{F G}\right\rangle_{A} \\
& =s^{2}\langle\overrightarrow{E G}, \overrightarrow{E G}\rangle_{A}=s^{2}\|\overrightarrow{E G}\|_{A}^{2} .
\end{aligned}
$$

Combining the above equation with Equation (3.135) yields

$$
\frac{\|\overrightarrow{A C}\|_{A}^{2}}{\|\overrightarrow{E G}\|_{A}^{2}}=s^{2}
$$

Taking the positive square root we obtain Equation (3.134), as desired.

Next, we show the angle-angle-angle (A.A.A.) similarity theorem of triangles.

Theorem 3.20. (Angle-Angle-Angle similarity in arrow spaces) Let $\triangle A B C, \triangle E F G$ be two triangles in an arrow space $\mathscr{P}_{A}$, as in Figure 3.20. If

$$
\cos \angle B A C=\cos \angle F E G, \cos \angle A B C=\cos \angle E F G, \cos \angle A C B=\cos \angle E G F,
$$

then $\triangle A B C, \triangle E F G$ are similar.

Proof : By assumption and Definition 27 we have

$$
\sin \angle B A C=\sin \angle F E G, \sin \angle A B C=\sin \angle E F G, \sin \angle A C B=\sin \angle E G F .
$$

We want to show that

$$
\frac{\|\overrightarrow{A B}\|_{A}}{\|\overrightarrow{E F}\|_{A}}=\frac{\|\overrightarrow{A C}\|_{A}}{\|\overrightarrow{E G}\|_{A}}=\frac{\|\overrightarrow{B C}\|_{A}}{\|\overrightarrow{F G}\|_{A}}
$$

Now, by Theorem 3.18 we have

$$
\frac{\|\overrightarrow{A B}\|_{A}}{\sin \angle A C B}=\frac{\|\overrightarrow{B C}\|_{A}}{\sin \angle B A C}=\frac{\|\overrightarrow{A C}\|_{A}}{\sin \angle A B C}
$$

and

$$
\frac{\sin \angle E G F}{\|\overrightarrow{E F}\|_{A}}=\frac{\sin \angle F E G}{\|\overrightarrow{F G}\|_{A}}=\frac{\sin \angle E F G}{\|\overrightarrow{E G}\|_{A}} .
$$

Equations (3.138) and (3.139) imply that

$$
\frac{\sin \angle E G F}{\|\overrightarrow{E F}\|_{A}} \frac{\|\overrightarrow{A B}\|_{A}}{\sin \angle A C B}=\frac{\sin \angle F E G}{\|\overrightarrow{F G}\|_{A}} \frac{\|\overrightarrow{B C}\|_{A}}{\sin \angle B A C}=\frac{\sin \angle E F G}{\|\overrightarrow{E G}\|_{A}} \frac{\|\overrightarrow{A C}\|_{A}}{\sin \angle A B C}
$$


By applying 3.136 to 3.140, we obtain Equation (3.137), that is

$$
\frac{\|\overrightarrow{A B}\|_{A}}{\|\overrightarrow{E F}\|_{A}}=\frac{\|\left.\overrightarrow{A C}\right|_{A}}{\|\overrightarrow{E G}\|_{A}}=\frac{\|\overrightarrow{B C}\|_{A}}{\|\overrightarrow{F G}\|_{A}}
$$

as desired.

Theorem 3.21. Let $\triangle A B C, \triangle E F G$ be two triangles in an arrow space $\mathscr{P}_{A}$, as in Figure 3.20. If

$$
\frac{\|\overrightarrow{A B}\|_{A}}{\|\overrightarrow{E F}\|_{A}}=\frac{\|\overrightarrow{A C}\|_{A}}{\|\overrightarrow{E G}\|_{A}}=\frac{\|\overrightarrow{B C}\|_{A}}{\|\overrightarrow{F G}\|_{A}}=s, s>0
$$

then

$$
\cos \angle B A C=\cos \angle F E G, \cos \angle A B C=\cos \angle E F G, \cos \angle A C B=\cos \angle E G F,
$$

Proof : Notice that, by Definitions 5, 6, Equations (1.8), (1.2), and (1.5) we can write

$$
\begin{aligned}
\|\overrightarrow{A C}\|_{A}^{2} & =\langle\overrightarrow{A C}, \overrightarrow{A C}\rangle_{A}=\left\langle\overrightarrow{A B}+{ }_{A} \overrightarrow{B C}, \overrightarrow{A B}+_{A} \overrightarrow{B C}\right\rangle_{A} \\
& =\|\overrightarrow{A B}\|_{A}^{2}+\|\overrightarrow{B C}\|_{A}^{2}+2\langle\overrightarrow{A B}, \overrightarrow{B C}\rangle_{A} \\
& =\|\overrightarrow{A B}\|_{A}^{2}+\|\overrightarrow{B C}\|_{A}^{2}-2\langle\overrightarrow{B A}, \overrightarrow{B C}\rangle_{A} .
\end{aligned}
$$

Similarly,

$$
s^{2}\|\overrightarrow{E G}\|_{A}^{2}=s^{2}\|\overrightarrow{E F}\|_{A}^{2}+s^{2}\|\overrightarrow{F G}\|_{A}^{2}-2 s^{2}\langle\overrightarrow{F E}, \overrightarrow{F G}\rangle_{A} .
$$

Now, it follows by the hypothesis, Equation (3.142), that

$$
\|\overrightarrow{A C}\|_{A}^{2}=s^{2}\|\overrightarrow{E G}\|_{A}^{2},\|\overrightarrow{A B}\|_{A}^{2}=\|\overrightarrow{E F}\|_{A}^{2},\|\overrightarrow{B C}\|_{A}^{2}=s^{2}\|\overrightarrow{F G}\|_{A}^{2} .
$$

If we plug in the preceding equation into equation 3.143, we get

$$
s^{2}\|\overrightarrow{E G}\|_{A}^{2}=s^{2}\|\overrightarrow{E F}\|_{A}^{2}+s^{2}\|\overrightarrow{F G}\|_{A}^{2}-2\langle\overrightarrow{B A}, \overrightarrow{B C}\rangle_{A} .
$$

If we combine 3.144 and 3.146 we conclude that

$$
\langle\overrightarrow{B A}, \overrightarrow{B C}\rangle_{A}=s^{2}\langle\overrightarrow{F E}, \overrightarrow{F G}\rangle_{A}
$$

Since by the hypothesis, Equation (3.142), we have that

$$
\|\overrightarrow{B A}\|_{A}\|\overrightarrow{B C}\|_{A}=s^{2}\|\overrightarrow{F E}\|_{A}\|\overrightarrow{F G}\|_{A}
$$


Dividing Equation (3.147) by Equation (3.148) yields

$$
\frac{\langle\overrightarrow{B A}, \overrightarrow{B C}\rangle_{A}}{\|\overrightarrow{B A}\|_{A}\|\overrightarrow{B C}\|_{A}}=\frac{s^{2}\langle\overrightarrow{F E}, \overrightarrow{F G}\rangle_{A}}{s^{2}\|\overrightarrow{F E}\|_{A}\|\overrightarrow{F G}\|_{A}}=\frac{\langle\overrightarrow{F E}, \overrightarrow{F G}\rangle_{A}}{\|\overrightarrow{F E}\|_{A}\|\overrightarrow{F G}\|_{A}}
$$

Combining this identity with Definition 18 shows that $\cos \angle A B C=\cos \angle E F G$. Similar arguments, which are left to the reader, show that $\cos \angle B A C=\cos \angle F E G$, and $\cos \angle A C B=\cos \angle E G F$.

\subsection{Axioms of Congruence in Arrow Spaces}

In Theorem 3.19 of Section 3.7, if $s=1$, then this theorem is the side-angle-side congruence theorem of triangles. It is the sixth axiom found in Hilbert's axioms, namely, Axiom C6. In other words, this axiom follows as a theorem in arrow spaces. In this section, we prove the five remaining axioms of congruence in arrow spaces. We start with line segment congruence by restating the definition of congruent line segments in terms of arrows.

Definition 29. Two arrows $\overrightarrow{A B}$ and $\overrightarrow{C D}$ are said to be congruent if $\|\overrightarrow{A B}\|_{A}=\|\overrightarrow{C D}\|_{A}$.

Theorem 3.3. (The Axiom $C 1$ of Congruence) Given an arrow $\overrightarrow{A B}$, and given a ray $\overrightarrow{C P}_{\infty}$ originating at a point $C$, there exists a unique arrow $\overrightarrow{C D}_{\text {with }} D \in \overrightarrow{C P}_{\infty}$ such that the two arrows $\overrightarrow{A B}, \overrightarrow{C D}$ are congruent.

Proof : Let $\|\overrightarrow{A B}\|_{A}=s>0$. Using Theorem 3.6, let $M \in \overrightarrow{C P}_{\infty}$ be such that the arrow $\overrightarrow{C M}$ is the unique unit arrow. By Definition 16 and Axiom 3 , there exists a unique point $D \in \overrightarrow{C P}_{\infty}$ such that $\overrightarrow{C D}={ }_{A}(s) \overrightarrow{C M}$. Now, since $\|\overrightarrow{A B}\|_{A}=s>0$, it follows by 1.9 that

$$
\|\overrightarrow{C D}\|_{A}=\|(s) \overrightarrow{C M}\|_{A}=s\|\overrightarrow{C M}\|_{A}=s=\|\overrightarrow{A B}\|_{A}
$$

The next axiom is about the transitivity and reflexivity of the congruence relation of arrows.

Theorem 3.4. (The Axiom $C 2$ of Congruence) If $\overrightarrow{A B}$ is congruent to $\overrightarrow{C D}$ and $\overrightarrow{C D}$ is congruent to $\overrightarrow{E F}$, then $\overrightarrow{A B}$ is congruent to $\overrightarrow{E F}$. Every arrow is congruent to itself.

Proof : The proof is immediate from Definition 29.

We need the following proposition to prove the next axiom. 
Proposition 3.13. Let $A, B, C$ be three distinct points that lie on a line. If the two arrows $\overrightarrow{A B}, \overrightarrow{B C}$ have the point $B$ as the only common point, then we must have that

$$
\frac{\langle\overrightarrow{B A}, \overrightarrow{B C}\rangle_{A}}{\|\overrightarrow{B A}\|_{A}\|\overrightarrow{B C}\|_{A}}=-1
$$

that is, $A \prec B \prec C$.

Proof : Since the three points $A, B$, and $C$ lie on the same line, it follows by Proposition 1.8 that

If

$$
\frac{\langle\overrightarrow{B A}, \overrightarrow{B C}\rangle_{A}}{\|\overrightarrow{B A}\|_{A}\|\overrightarrow{B C}\|_{A}}= \pm 1
$$

$$
\frac{\langle\overrightarrow{B A}, \overrightarrow{B C}\rangle_{A}}{\|\overrightarrow{B A}\|_{A}\|\overrightarrow{B C}\|_{A}}=-1,
$$

then this is exactly the desired equation 3.149 , and we are done. Thus, assume, by way of contradiction, that

$$
\frac{\langle\overrightarrow{B A}, \overrightarrow{B C}\rangle_{A}}{\|\overrightarrow{B A}\|_{A}\|\overrightarrow{B C}\|_{A}}=1,
$$

Now, the preceding equation implies, by means of Theorem 1.9 Part 3, that there exists $t>0$ such that

$$
\overrightarrow{B A}={ }_{A}(t) \overrightarrow{B C}, t>0
$$

We consider two possibles cases for $t$, namely $t<1$ and $t>1$.

Case 1. If $t<1$ in Equation 3.151, this means by Theorem 3.1 that $B \prec A \prec C$. This means that $A$ is another common point other than $B$ that the two arrows $\overrightarrow{A B}, \overrightarrow{B C}$ share, which is a contradiction to the hypothesis of this proposition.

Case 2. If $t>1$, then we have by Theorem 3.4 that $B \prec C \prec A$. This means again that there are two points, in this case $B$ and $C$, in common between the two arrows $\overrightarrow{A B}, \overrightarrow{B C}$, which contradicts the hypothesis, too. Thus, Equation 3.150 is impossible and we must have Equation 3.149, as desired.

Now, we prove the analog of the third axiom of congruence in arrow spaces.

Theorem 3.5. (The Axiom $C 3$ of Congruence) On the line let $\overrightarrow{A B}$ and $\overrightarrow{B C}$ be two arrows which except for $B$ have no point in common. Furthermore, on the same or on another line $\ell^{\prime}$ let $\overrightarrow{A^{\prime} B^{\prime}}$ and $\overrightarrow{B^{\prime} C^{\prime}}$ be two arrows which except for $B^{\prime}$ have no point in common. In the case, if $\overrightarrow{A B}$ is congruent to $\overrightarrow{A^{\prime} B^{\prime}}$ and $\overrightarrow{B C}$ is congruent to $\overrightarrow{B^{\prime} C^{\prime}}$, then $\overrightarrow{A C}$ is congruent to $\overrightarrow{A^{\prime} C^{\prime}}$. 
Proof : Let $\overrightarrow{A B}$ and $\overrightarrow{B C}$ be two arrows which except for $B$ have no point in common, where $A, B, C \in l$ and $A^{\prime}, B^{\prime}, C^{\prime} \in l^{\prime}$. Since $\overrightarrow{A B}$ is congruent to $\overrightarrow{A^{\prime} B^{\prime}}$ and $\overrightarrow{B C}$ is congruent to $\overrightarrow{B^{\prime} C^{\prime}}$, it follows by Definition 29 that

$$
\|\overrightarrow{A B}\|_{A}=\left\|\overrightarrow{A^{\prime} B^{\prime}}\right\|_{A}=s \text { and }\|\overrightarrow{B C}\|_{A}=\left\|\overrightarrow{B^{\prime} C^{\prime}}\right\|_{A}=t
$$

Notice that, since $A, B, C \in l$ and since $B$ is the only common point between $\overrightarrow{A B}$ and $\overrightarrow{B C}$, it follows by Proposition 3.13 that $A \prec B \prec C$. Similarly, $A^{\prime} \prec B^{\prime} \prec C^{\prime}$. This means that we have

$$
\frac{\langle\overrightarrow{B A}, \overrightarrow{B C}\rangle_{A}}{\|\overrightarrow{B A}\|_{A}\|\overrightarrow{B C}\|_{A}}=-1, \text { and } \frac{\left\langle\overrightarrow{B^{\prime} A^{\prime},}, \overrightarrow{B^{\prime} C^{\prime}}\right\rangle_{A}}{\left\|\overrightarrow{B^{\prime} A^{\prime}}\right\|_{A}\left\|\overrightarrow{B^{\prime} C^{\prime}}\right\|_{A}}=-1 \text {. }
$$

The equations in the above mean, according to Theorem 1.10 Part 2, that

$$
\|\overrightarrow{A C}\|_{A}=\|\overrightarrow{A B}\|_{A}+\|\overrightarrow{B C}\|_{A} \text {, and }\left\|\overrightarrow{A^{\prime} C^{\prime}}\right\|_{A}=\left\|\overrightarrow{A^{\prime} B^{\prime}}\right\|_{A}+\left\|\overrightarrow{B^{\prime} C^{\prime}}\right\|_{A}
$$

Combining the above equations and Equation 3.152 yields

$$
\|\overrightarrow{A C}\|_{A}=\left\|\overrightarrow{A^{\prime} C^{\prime}}\right\|_{A}=s+t
$$

This means, by Definition 29 , that $\overrightarrow{A C}$ is congruent to $\overrightarrow{A^{\prime} C^{\prime}}$, as desired.

Since the two following axioms are related to angles' congruent, we give a definition of congruent angles in arrow spaces.

Definition 30. Two angles $\angle A B C, \angle E F G$ are said to be congruent, denoted by $\angle A B C \cong \angle E F G$, in an arrow space if $\cos \angle A B C=\cos \angle E F G$.

Next, we prove the following axiom which is about laying off angles.

Theorem 3.6. (The Axiom $C 4$ of Congruence) Given an angle $\angle B A C$ and given a ray $\overrightarrow{D F}_{\infty}$, there exists a unique ray $\overrightarrow{D E}_{\infty}$ on a given side of the line $l_{D F}$, such that $\angle B A C \cong \angle E D F$.

Proof : Let an angle $\angle B A C$ and a ray $\overrightarrow{D F}_{\infty}$ be given. Using Theorem 3.10, let $S_{1}$ be a given side of the line $l_{D F}$ and $H$ be any point in $S_{1}$. Let $L \in l_{D F}$ be such that $\overrightarrow{D L}={ }_{A}(-1) \overrightarrow{D F}$. Then, the three points $L, F, H$ are distinct. Consider the triangle $\triangle L E F$, see Figure 3.21. Then, it follows by the setting of the proof of Theorem 3.10 that for every point $P$ such that $A \prec P \prec H$ or $H \prec P \prec F$, we must have $P \in S_{1}$. Now, we consider the two following cases. 

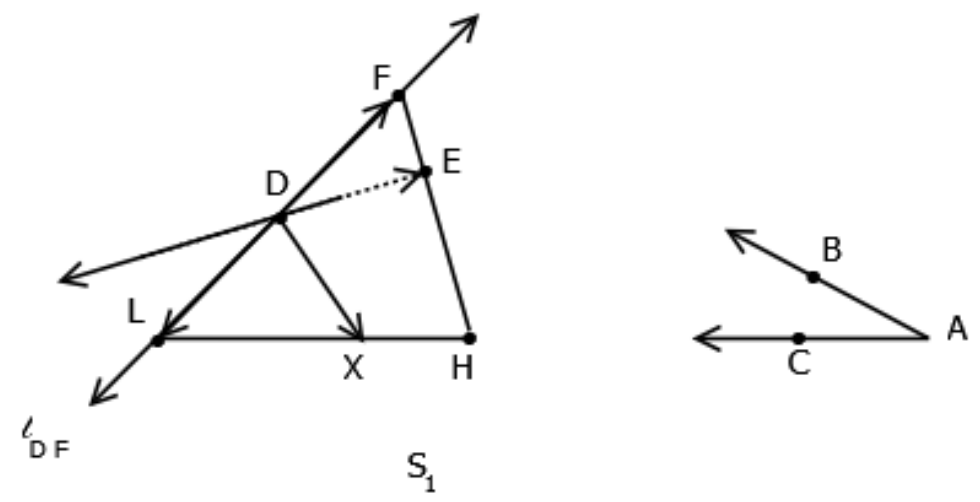

Figure 3.21: An illustration of Theorem 3.6 for laying off angles.

Case 1. If $\cos \angle H D F=\cos \angle B A C$, then we put $E={ }_{P} H$ to get the desired ray $\overrightarrow{D E}_{\infty}$ and we are done.

Case 2. If $\cos \angle H D F \neq \cos \angle B A C$, we apply Theorem 3.1 to the triangle $\triangle L E H$, where we put $\psi=\angle B A C$ to conclude that there exists a unique point $E$ either between $H, L$ or between $H, F$ such that $\cos \angle E D F=\cos \angle B A C$, as desired.

The last axiom affirms that the congruence relation of angles is transitive and reflexive. The proof is straightforward and follows from the Definition of congruent angles.

Theorem 3.7. (The Axiom $C 5$ of Congruence)

If $\angle B A C \cong \angle E D F$ and $\angle B A C \cong \angle L M N$, then $\angle L M N \cong \angle E D F$. Every angle is congruent to itself.

Proof : The proof is immediate from Definition 30. 


\section{Chapter 4}

\section{Trigonometry in Complex Inner Product Spaces}

The purpose of this chapter is solving problems in Geometry using arrows and vectors. We adopt an appropriate definition of an angle in a Hilbert space over the complex field. It serves as a main tool for the enhancement of geometry and trigonometry of complex inner product spaces. A "sine theorem" is shown to hold based on which similarity theorems are proven. Trigonometric identities are shown to hold that lead to a main result that the sum of angles in a "triangle" in a complex Hilbert space is $\pi$. Examples are given.

\subsection{An Appropriate Definition of The Cosine of An Angle in Complex Hilbert Spaces}

Hilbert spaces over the field of complex numbers play an important role in mathematical physics. Witness their relevance to the foundation of quantum mechanics. See F. Gallone [18] and the references there in. Compare also with Carl M. Bender et al. [7] and the references there in. The purpose of this chapter is to equip complex Hilbert spaces with a structure analogous to the structure of Euclidean geometry and trigonometry. By doing so, we obtain new tools for the relative estimate and for the relative comparison of ratios of norms of elements in a complex Hilbert space. Of particular interest are vector spaces of operators and function spaces over the field of complex numbers. Central to our study is a proper choice of a measure of an angle in a complex Hilbert space.

The importance of angles in Euclidean geometry, in Hilbert spaces, and in the mathematical sciences can hardly be overestimated. While the definition of an angle and its measure in Euclidean geometry and in a real Hilbert space is well established 
and accepted, the same does not hold true in a real Banach space and in a complex Hilbert space. The latter were subject to numerous investigations. A partial list includes Scharnhorst [23]; J. E. Valentine and S. G. Wayment [27]; A. Galantai and Cs. J. Hegedus [15]; and more recently V. W. Thurey [26]; V. Balestro, H. Martini, and R. Teixeira [5] and their references. It is yet to be determined which of the definitions of a measure of an angle in a complex Hilbert space is most appropriate and useful.

Let the vectors $\vec{x}, \vec{y}$ be represented by two arrows emanating from the same point. Let the arrows representing the three vectors $\vec{x}, \vec{y}$, and $\vec{x}-\vec{y}$ form a triangle. We assume throughout this chapter that the fundamental functions $\cos \theta$ and $\sin \theta$ and their properties follow from their representation as Taylor series as given in calculus. Given an inner product space over the field of complex numbers, we use throughout this chapter the definition

$$
\cos \angle \theta(\vec{x}, \vec{y})=\cos \theta(\vec{x}, \vec{y}):=\frac{\langle\vec{x}, \vec{y}\rangle+\langle\vec{y}, \vec{x}\rangle}{2\|\vec{x}\|\|\vec{y}\|},
$$

where $\angle \theta(\vec{x}, \vec{y})$ denotes the angle domain between vectors $\vec{x}$ and $\vec{y}$. Notice that $\cos \theta(\vec{x}, \vec{y})$ is a monotone decreasing function on the interval $0 \leq \theta \leq \pi$ and hence invertible. It is readily observed that $\cos \theta(\vec{x}, \vec{y})=\operatorname{Re}\langle\vec{x}, \vec{y}\rangle$ and is real even in a complex Hilbert space. Moreover, in a real Hilbert space, Definition 4.1 is compatible with the definition of the measure of an angle in a real Hilbert space. Furthermore, in $\mathbb{R}^{3}$ it is consistent with the definition of the measure of an angle in Euclidean geometry. It is noteworthy that Hartshorne [[20], pp. 141-142] defines the measure of an angle via the tangent function rather than by the cosine function. A confirmation to the utility of Definition 4.1 is provided by H. Gingold et al. [19]. With Definition 4.1, it was shown in [19] that the projection of a complex Hilbert space onto bowl-shaped subsets of a sphere is conformal if and only if the projection is stereographic.

It is noteworthy that if $\vec{x}, \vec{y}$ are unit vectors, then

$$
\cos \theta(\vec{x}, \vec{y}):=\frac{\langle\vec{x}, \vec{y}\rangle+\langle\vec{y}, \vec{x}\rangle}{2}
$$

is obtained as the coefficient of the linear term in the expansion of $\|\vec{y}+\delta \vec{x}\|$ as a Taylor series about $\delta=0$. Thus, cementing the fact that $\cos \theta(\vec{x}, \vec{y})$ is derived from the variation function of the magnitude of the vector $\vec{x}$ about the vector $\vec{y}$. Indeed, with $a=\langle\vec{x}, \vec{y}\rangle+\langle\vec{y}, \vec{x}\rangle$ we have

$$
\langle\vec{y}+\delta \vec{x}, \vec{y}+\delta \vec{x}\rangle^{\frac{1}{2}}=\left[1+a \delta+\delta^{2}\right]^{\frac{1}{2}}=1+\frac{1}{2}\left(a \delta+\delta^{2}\right)+\sum_{k=2}^{\infty}\left(\begin{array}{c}
\frac{1}{2} \\
k
\end{array}\right)\left(a \delta+\delta^{2}\right)^{k},
$$


with the series being absolutely convergent for $\delta$ small enough.

There are additional reasons to accept Definition 4.1 as a natural and useful definition and to prefer it to the textbooks' definition of $\cos \theta(\vec{x}, \vec{y}):=\frac{\langle\vec{x}, \vec{y}\rangle}{\|\vec{x}\|\|\vec{y}\|}$. Compare with S. Axler [4]. In an inner product space over the complex numbers, the definition $\cos \theta(\vec{x}, \vec{y})=\frac{\langle\vec{x}, \vec{y}\rangle}{\|\vec{x}\|\|\vec{y}\|}$ creates ambiguity. The ambiguity stems from the fact that we may have $\langle\vec{x}, \vec{y}\rangle \neq\langle\vec{y}, \vec{x}\rangle$, and consequently, we could end up with

$$
\cos \theta(\vec{x}, \vec{y})=\overline{\cos \theta(\vec{y}, \vec{x})}=\frac{\langle\vec{x}, \vec{y}\rangle}{\|\vec{x}\|\|\vec{y}\|} \neq \cos \theta(\vec{y}, \vec{x})=\frac{\langle\vec{y}, \vec{x}\rangle}{\|\vec{x}\|\|\vec{y}\|}
$$

Let

$$
\vec{x}:=\left(x_{1}, x_{2}, \ldots, x_{n}\right) \in \mathbb{R}^{n}, \vec{y}:=\left(y_{1}, y_{2}, \ldots, y_{n}\right) \in \mathbb{R}^{n}, x_{j}, y_{j} \in \mathbb{R} \text { for } j=1,2, \ldots, n,
$$

and consider the Euclidean inner product $\langle x, y\rangle_{E}:=\sum_{j=1}^{n} x_{j} y_{j}$. It is well known that if one defines the cosine of the measure $\theta(\vec{x}, \vec{y})$ of an angle between two vectors $\vec{x}$ and $\vec{y}$ to be $\cos \theta(\vec{x}, \vec{y})=\frac{\langle\vec{x}, \vec{y}\rangle_{E}}{\|\vec{x}\| \|}$, then this value for $n=2,3$ coincides with the value of $\cos \theta(\vec{x}, \vec{y})$ as defined in elementary trigonometry. However, to the best of our knowledge, there is no guarantee that the elementary theorems of geometry and the trigonometric identities hold in a real Hilbert space with a general inner product. The more so if it is a complex Hilbert space. This chapter is also to fill this gap.

Put

$$
\langle\vec{x}, \vec{y}\rangle=\rho \exp i \theta, \rho \geq 0,0 \leq \theta \leq 2 \pi .
$$

The important concept of orthogonality of $\vec{x}$ and $\vec{y}$ as defined by textbooks as $\langle\vec{x}, \vec{y}\rangle=0$, namely $\rho=0$, implies orthogonality in the sense that

$$
\langle\vec{x}, \vec{y}\rangle+\langle\vec{y}, \vec{x}\rangle=0
$$

However, if $\langle\vec{x}, \vec{y}\rangle+\langle\vec{y}, \vec{x}\rangle=0$, this does not imply that $\langle\vec{x}, \vec{y}\rangle=\langle\vec{y}, \vec{x}\rangle=0$. Then,

$$
\langle\vec{x}, \vec{y}\rangle+\langle\vec{y}, \vec{x}\rangle=\rho \exp i \theta+\rho \exp (-i \theta)=2 \rho \cos \theta .
$$

So, $\rho>0$ is possible and then $\langle\vec{x}, \vec{y}\rangle+\langle\vec{y}, \vec{x}\rangle=0$ implies that $\theta=\frac{\pi}{2}$, or $\frac{3 \pi}{2}$.

Similarity is an important concept in numerous applications. See Hartshorne [[20], Chap. 4, Sect. 20]. Similarity theorems in Euclidean geometry play a very important role. They underline the development of trigonometry, its definitions, and its identities. They beg the question, does there exist an analog to trigonometry in complex valued Hilbert spaces? The answer is yes if one adopts Definition 4.1. It is also shown in this chapter that congruence theorems between triangles are a special 
case of similarity theorems. Moreover, it is shown in this chapter that a "cosine theorem" holds in complex Hilbert spaces thanks to Definition 4.1. Furthermore, it is shown that a "cosine theorem" in complex Hilbert spaces holds if and only if a corresponding "sine theorem" holds. This is given in Section 4.2. It follows then that the property of similarity of triangles is invariant with respect to isometries.

As an application of the sine theorem we have

Example 2. Consider the space of all continuous complex-valued functions on $[a, b]$ with inner product defined by $\langle f, g\rangle=\int_{a}^{b} f(s) \bar{g}(s) d s$ where $f, g \in C[a, b]$ and $a, b$ are real numbers. Assume

$$
\int_{a}^{b} f(s) \bar{f}(s) d s>0 \quad \text { and } \quad \int_{a}^{b} g(s) \bar{g}(s) d s>0 .
$$

Then,

$$
\frac{\sin ^{2} \theta(f, g)}{\int_{a}^{b}(f(s)-g(s))(\bar{f}(s)-\bar{g}(s)) d s}=\frac{\sin ^{2} \theta(f-g, f)}{\int_{a}^{b} g(s) \bar{g}(s) d s}=\frac{\sin ^{2} \theta(g-f, g)}{\int_{a}^{b} f(s) \bar{f}(s) d s} .
$$

Example 3. Let $\operatorname{Mat}(n, n, \mathbb{C})$ be the vector space of $n \times n$ complex matrices with the Frobenius inner product given by

$$
\langle A, B\rangle=\operatorname{trace}\left(A^{*} B\right), \quad A, B \in \operatorname{Mat}(n, n, \mathbb{C}) .
$$

The sine theorem implies that

$$
\frac{\sin ^{2} \theta(A, B)}{\operatorname{trace}\left((A-B)^{*}(A-B)\right)}=\frac{\sin ^{2} \theta(A-B, A)}{\operatorname{trace}\left(B^{*} B\right)}=\frac{\sin ^{2} \theta(B-A, B)}{\operatorname{trace}\left(A^{*} A\right)} .
$$

In Section 4.4, it is shown that with Definition 4.1, the identities of elementary trigonometry hold in complex Hilbert spaces. Consequently, a theorem is proven in Section 4.4 that the sum of angles in a triangle in a complex Hilbert space is $\pi$. Thanks to this we conclude that a triangle in a complex Hilbert space must have at least two acute angles in the sense of Definition 4.1. This has ramifications on projections in any Hilbert space as follows.

Consider a triangle with sides being arrows representing the vectors $\vec{x}, \vec{y}$, and $\vec{x}-\vec{y}$ in a complex Hilbert space. See Figure 4.1.

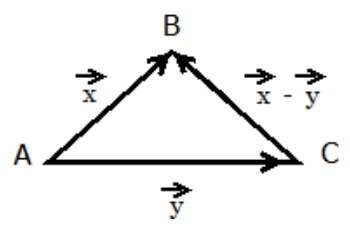

Figure 4.1: Projections 
Consider the cosines of the three angles according to convention Definition 4.1. Then

- Two out of three cosines below must be nonnegative

$$
\cos \theta(-\vec{x}, \vec{y}-\vec{x}) \quad \cos \theta(\vec{x}-\vec{y},-\vec{y}) \quad \cos \theta(\vec{y}, \vec{x}) .
$$

- Two of the components out of the three below must be nonnegative

$$
\begin{gathered}
\|\vec{x}\| \cos \theta(-\vec{x}, \vec{y}-\vec{x}),\|\vec{y}-\vec{x}\| \cos \theta(\vec{x}-\vec{y},-\vec{y}), \text { and } \\
\|\vec{y}\| \cos \theta(\vec{y}, \vec{x}) .
\end{gathered}
$$

- Evidently, the three projections below

$$
\begin{aligned}
& \|\vec{x}\| \cos \theta(-\vec{x}, \vec{y}-\vec{x}) \frac{\vec{x}-\vec{y}}{\|\vec{x}-\vec{y}\|} \\
& \|\vec{y}-\vec{x}\| \cos \theta(\vec{x}-\vec{y},-\vec{y}) \frac{\frac{\vec{y}}{\|\vec{y}\|}}{\|\vec{y}\| \cos \theta(\vec{y}, \vec{x}) \frac{\vec{x}}{\|\vec{x}\|},}
\end{aligned}
$$

are collinear with the $\vec{x}-\vec{y}, \vec{y}$, and $\vec{x}$, respectively. However, two out of the three projections above must have the same directions as the directions of $\vec{x}-\vec{y}, \vec{y}$, and $\vec{x}$, respectively. It is possible to verify this independently by simple arguments.

It goes without saying that our adoption of Definition 4.1 leads to the construction of a projection that is different than traditional projection of a vector $\vec{x}$ on a vector $\vec{y}$ in a complex Hilbert space. The central result of this chapter, namely that the sum of the measures of the three angles in a triangle of complex Hilbert space is $\pi$, provides an alternative proof that the shortest distance from a point to a line is unique.

\subsection{Cosine and Sine Theorems in Complex Hilbert Spaces}

Let $\mathbb{X}$ be a complex Hilbert space. We must first define what we mean by an angle between two vectors of $\mathbb{X}$. Let $\vec{x}$ and $\vec{y}$ be two nonzero vectors in $\mathbb{X}$ emanating from a fixed origin $O$. Let $\mathbb{P}$ be the two dimensional subspace in $\mathbb{X}$ determined by $\vec{x}$ and $\vec{y}$. Let

$$
l_{x}:=t \vec{x}, t \geq 0 \quad l_{y}:=t \vec{y}, t \geq 0 .
$$




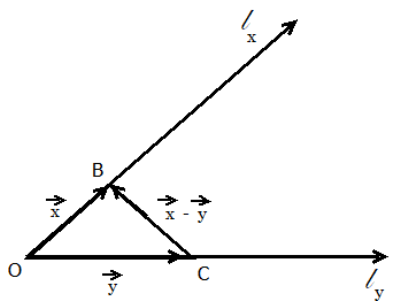

Figure 4.2: Definition of an Angle

Geometrically, as shown in Figure 4.2, $l_{x}$ and $l_{y}$ are the rays in the directions of $\vec{x}$ and $\vec{y}$ which determine the sides of the angle $\angle \theta(\vec{x}, \vec{y})$. We define $\angle \theta(\vec{x}, \vec{y})$ to be the convex subset of $\mathbb{P}$ between $l_{x}$ and $l_{y}$ determined by positive combinations of $\vec{x}$ and $\vec{y}$, i.e.

$$
\angle \theta(\vec{x}, \vec{y}):=\{\alpha \vec{x}+\beta \vec{y} \mid \alpha, \beta>0\} .
$$

We next define the cosine function of an angle to be the real part of the inner product of the two vectors which determine the sides of the angle. This ensures that the cosine is always a real number. Furthermore, this definition of cosine is consistent with the cosine theorem as defined in elementary trigonometry.

We should remark that our definition of cosine avoids reference to angle measure. This is no accident since V. Balestro et al. [6] and M. Obst [22] have shown that in an abstract Hilbert space, the definition of angle measure is nontrivial and not "natural"

Definition 31. Let $\vec{x}$ and $\vec{y}$ be two nonzero vectors in a complex Hilbert space $\mathbb{X}$. Let $\angle \theta(\vec{x}, \vec{y})$ denote the angle region as defined by Equation (4.2). See Figure 4.2. Define a cosine function of $\angle \theta(\vec{x}, \vec{y})$ as

$$
\cos \angle \theta(\vec{x}, \vec{y})=\cos \theta(\vec{y}, \vec{x}):=\frac{\langle\vec{x}, \vec{y}\rangle+\langle\vec{y}, \vec{x}\rangle}{2\|\vec{x}\|\|\vec{y}\|} .
$$

Proposition 4.1. The cosine function of $\angle \theta(\vec{x}, \vec{y})$ given by Definition 31 has the following properties:

1. $|\cos \theta(\vec{x}, \vec{y})| \leq 1$,

2. $\cos \theta(\vec{x}, \vec{y})=\cos \theta(-\vec{x},-\vec{y})$

Proof : By Cauchy-Schwarz inequality in $\mathbb{X}$ we have,

$$
\begin{aligned}
|\cos \theta(\vec{x}, \vec{y})| & =\left|\frac{\langle\vec{x}, \vec{y}\rangle+\langle\vec{y}, \vec{x}\rangle}{2\|\vec{x}\|\|\vec{y}\|}\right| \leq \frac{|\langle\vec{x}, \vec{y}\rangle|+|\langle\vec{y}, \vec{x}\rangle|}{2\|\vec{x}\|\|\vec{y}\|} \\
& \leq \frac{\|\vec{x}\|\|\vec{y}\|+\|\vec{x}\|\|\vec{y}\|}{2\|\vec{x}\|\|\vec{y}\|}=1 .
\end{aligned}
$$

Property 2 follows immediately from (4.3) in Definition 31. 
With the aid of Definition 31, we show that the cosine theorem holds in the complex Hilbert space $\mathbb{X}$.

Proposition 4.2. (The Cosine Theorem for Complex Hilbert Spaces) Let $\mathbb{X}$ be a complex Hilbert space. Let $\vec{x}, \vec{y}$, and $\vec{x}+\vec{y}$ be three nonzero vectors of $\mathbb{X}$ which form Triangle $A B C$, as illustrated in Figure 4.3. Then,

$$
\|\vec{x}+\vec{y}\|^{2}=\|\vec{x}\|^{2}+\|\vec{y}\|^{2}-2\|\vec{x}\|\|\vec{y}\| \cos \theta(-\vec{x}, \vec{y})
$$

if and only if $\cos \theta(\vec{x}, \vec{y})=\frac{\langle\vec{x}, \vec{y}\rangle+\langle\vec{y}, \vec{x}\rangle}{2\|\vec{x}\|\|\vec{y}\|}$.

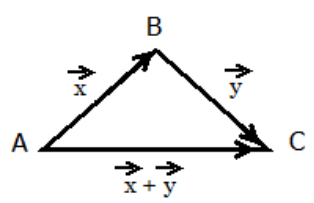

Figure 4.3: The Cosine Theorem

Proof : Notice that

$$
\begin{aligned}
\|\vec{x}+\vec{y}\|^{2} & =\langle\vec{x}+\vec{y}, \vec{x}+\vec{y}\rangle \\
& =\|\vec{x}\|^{2}+\|\vec{y}\|^{2}-2\|\vec{x}\|\|\vec{y}\| \frac{\langle-\vec{x}, \vec{y}\rangle+\langle\vec{y},-\vec{x}\rangle}{2\|\vec{x}\|\|\vec{y}\|} .
\end{aligned}
$$

If Definition 31 holds, Equation (4.5) becomes Equation (4.4). On the other hand, applying the cosine theorem from classical trigonometry to the triangle of Figure 4.3 gives us Equation (4.4). Therefore, if we compare Equation (4.4) with Equation (4.5), we deduce that $\cos \theta(\vec{x}, \vec{y})=\frac{\langle\vec{x}, \vec{y}\rangle+\langle\vec{y}, \vec{x}\rangle}{2\|\vec{x}\|\|\vec{y}\|}$.

Definition 32. Let $\vec{x}$ and $\vec{y}$ be two nonzero vectors in a complex Hilbert space $\mathbb{X}$. Let $\angle \theta(\vec{x}, \vec{y})$ be the angle region between $\vec{x}$ and $\vec{y}$ as defined by 4.2. Define a sine function of $\angle \theta(\vec{x}, \vec{y})$ as

$$
\sin \angle \theta(\vec{x}, \vec{y})=\sin \theta(\vec{x}, \vec{y}):=\sqrt{1-\cos ^{2} \theta(\vec{x}, \vec{y})} .
$$

Property 1 of Proposition 4.1 shows that $0 \leq \sin \theta(\vec{x}, \vec{y}) \leq 1$.

We next show that the sine theorem holds for a complex Hilbert space $\mathbb{X}$. 
Theorem 4.1. (The Sine Theorem for a Complex Hilbert Space) Let $\mathbb{X}$ be a complex Hilbert space. Let $\vec{x}, \vec{y}$, and $\vec{x}-\vec{y}$ be three nonzero vectors of $\mathbb{X}$ which form Triangle $A B C$, as illustrated in Figure 4.1. Then,

$$
\begin{gathered}
\cos \theta(\vec{x}, \vec{y})=\frac{\langle\vec{x}, \vec{y}\rangle+\langle\vec{y}, \vec{x}\rangle}{2\|\vec{x}\|\|\vec{y}\|} \text { if and only if } \\
\frac{\sin \theta(\vec{x}, \vec{y})}{\|\vec{y}-\vec{x}\|}=\frac{\sin \theta(\vec{x}-\vec{y}, \vec{x})}{\|\vec{y}\|}=\frac{\sin \theta(\vec{y}-\vec{x}, \vec{y})}{\|\vec{x}\|}=q(x, y),
\end{gathered}
$$

where

$$
q^{2}(x, y)=\frac{4\|\vec{x}\|^{2}\|\vec{y}\|^{2}-a^{2}}{4\|\vec{x}\|^{2}\|\vec{y}\|^{2}\|\vec{y}-\vec{x}\|^{2}}, \quad a=\langle\vec{x}, \vec{y}\rangle+\langle\vec{y}, \vec{x}\rangle .
$$

Proof : Let $\cos \theta(\vec{x}, \vec{y})=\frac{\langle\vec{x}, \vec{y}\rangle+\langle\vec{y}, \vec{x}\rangle}{2\|\vec{x}\|\|\vec{y}\|}$. We want to show that Equation (4.7) holds. We will show that

$$
\frac{\sin \theta(\vec{x}, \vec{y})}{\|\vec{x}-\vec{y}\|}=\frac{\sin \theta(\vec{x}-\vec{y}, \vec{x})}{\|\vec{y}\|}
$$

and the proof of the similar identity

$$
\frac{\sin \theta(\vec{x}-\vec{y}, \vec{x})}{\|\vec{y}\|}=\frac{\sin \theta(\vec{y}-\vec{x}, \vec{y})}{\|\vec{x}\|}
$$

should follow by a similar argument. To prove Equation (4.8), it is enough to show that

$$
\frac{\sin ^{2} \theta(\vec{x}, \vec{y})}{\|\vec{x}-\vec{y}\|^{2}}=\frac{\sin ^{2} \theta(\vec{x}-\vec{y}, \vec{x})}{\|\vec{y}\|^{2}}
$$

since Definition 32 implies that the sine function has nonnegative range. Furthermore, Definition 32 implies that 4.8 is equivalent to

$$
\frac{1-\cos ^{2} \theta(\vec{x}, \vec{y})}{\|\vec{x}-\vec{y}\|^{2}}=\frac{1-\cos ^{2} \theta(\vec{x}-\vec{y}, \vec{x})}{\|\vec{y}\|^{2}} .
$$

Thus, in order to show 4.8 we need only to show that

$$
\|\vec{y}\|^{2}\left[1-\cos ^{2} \theta(\vec{x}, \vec{y})\right]=\|\vec{x}-\vec{y}\|^{2}\left[1-\cos ^{2} \theta(\vec{x}-\vec{y}, \vec{x})\right] .
$$

Notice that

$$
\begin{aligned}
\cos ^{2} \theta & (\vec{x}-\vec{y}, \vec{x})=\frac{[\langle\vec{x}, \vec{x}-\vec{y}\rangle+\langle\vec{x}-\vec{y}, \vec{x}\rangle]^{2}}{[2\|\vec{x}\|\|\vec{x}-\vec{y}\|]^{2}} \\
& =\frac{\left[2\|\vec{x}\| \|^{2}-\langle\vec{x}, \vec{y}\rangle-\langle\vec{y}, \vec{x}\rangle\right]^{2}}{[2\|\vec{x}\|\|\vec{x}-\vec{y}\|]^{2}}=\frac{\left[2\|\vec{x}\| \|^{2}-a\right]^{2}}{[2\|\vec{x}\|\|\vec{x}-\vec{y}\|]^{2}} .
\end{aligned}
$$


Thus,

$$
\begin{aligned}
\|\vec{x}-\vec{y}\|^{2}\left[1-\cos ^{2} \theta(\vec{x}-\vec{y}, \vec{x})\right]=\|\vec{x}-\vec{y}\|^{2}-\frac{\left[2\|\vec{x}\|^{2}-a\right]^{2}}{4\|\vec{x}\|^{2}} \\
=\frac{4\|\vec{x}\|^{2}\|\vec{x}-\vec{y}\|^{2}-\left[2\|\vec{x}\|^{2}-a\right]^{2}}{4\|\vec{x}\|^{2}} \\
=\frac{4\|\vec{x}\|^{2}\left(\|\vec{x}\|^{2}+\|\vec{y}\| \|^{2}-a\right)-\left[2\|\vec{x}\|^{2}-a\right]^{2}}{4\|\vec{x}\|^{2}} .
\end{aligned}
$$

Rearranging terms in the above yields

$$
\begin{aligned}
\|\vec{x}-\vec{y}\|^{2}\left[1-\cos ^{2} \theta(\vec{x}-\vec{y}, \vec{x})\right] & =\frac{4\|\vec{x}\|^{2}\|\vec{y}\|^{2}-a^{2}}{4\|\vec{x}\|^{2}} \\
& =\|\vec{y}\|^{2}-\frac{a^{2}}{4\|\vec{x}\|^{2}} \\
& =\|\vec{y}\|^{2}-\|\vec{y}\|^{2} \frac{[\langle\vec{x}, \vec{y}\rangle+\langle\vec{y}, \vec{x}\rangle]^{2}}{[2\|\vec{x}\|\|\vec{y}\|]^{2}} \\
& =\|\vec{y}\|^{2}\left[1-\cos ^{2} \theta(\vec{x}, \vec{y})\right],
\end{aligned}
$$

which is precisely the identity of Equation (4.9).

Observe that when verifying Equation (4.9), we showed that

$$
\|\vec{y}\|^{2} \sin ^{2} \theta(\vec{x}, \vec{y})=\frac{4\|\vec{x}\|^{2}\|\vec{y}\|^{2}-a^{2}}{4\|\vec{x}\|^{2}} .
$$

This in turn leads to the auxiliary identity

$$
\frac{\sin ^{2} \theta(\vec{x}, \vec{y})}{\|\vec{x}-\vec{y}\|^{2}}=\frac{1-\cos ^{2} \theta(\vec{x}, \vec{y})}{\|\vec{x}-\vec{y}\|^{2}}=\frac{4\|\vec{x}\|^{2}\|\vec{y}\|^{2}-a^{2}}{4\|\vec{x}\|^{2}\|\vec{y}\|^{2}\|\vec{x}-\vec{y}\|^{2}} .
$$

Now assume that Equation (4.7) holds. Then,

$$
q^{2}(x, y)=\frac{\sin ^{2} \theta(\vec{x}, \vec{y})}{\|\vec{y}-\vec{x}\|^{2}}=\frac{\sin ^{2} \theta(\vec{x}, \vec{y})}{\|\vec{y}-\vec{x}\|^{2}}=\frac{4\|\vec{x}\|^{2}\|\vec{y}\|^{2}-a^{2}}{4\|\vec{x}\|^{2}\|\vec{y}\|^{2}\|\vec{y}-\vec{x}\|^{2}} .
$$

This means

$$
\sin ^{2} \theta(\vec{x}, \vec{y})=1-\cos ^{2} \theta(\vec{x}, \vec{y})=\frac{4\|\vec{x}\|^{2}\|\vec{y}\|^{2}-a^{2}}{4\|\vec{x}\|^{2}\|\vec{y}\|^{2}}
$$

which in turn implies $\cos ^{2} \theta(\vec{x}, \vec{y})=\frac{a^{2}}{4\|\vec{x}\|^{2}\|\vec{y}\|^{2}}$, as desired. 


\subsection{Similarity of Triangles in Complex Hilbert Spaces.}

We start with the definition of similar triangles in a complex inner product spaces $\mathbb{X}$ and then prove the similarity theorems of triangles in $\mathbb{X}$.

Definition 33. Let $\mathbb{X}$ be a complex Hilbert space and let $A B C, E F G$ be two triangles in $\mathbb{X}$, their sides determined by the nonzero vectors $\vec{x}, \vec{y}, \vec{x}+\vec{y}$ and $\overrightarrow{x^{\prime}}, \overrightarrow{y^{\prime}}, \overrightarrow{x^{\prime}}+\overrightarrow{y^{\prime}}$, respectively, as illustrated in Figure 4.4. We say that Triangle $A B C$ is similar to Triangle $E F G$ if the corresponding sides are in proportion. That is, there exists a positive real number $s$ such that

$$
\frac{\|\vec{x}\|}{\left\|\overrightarrow{x^{\prime}}\right\|}=\frac{\|\vec{y}\|}{\left\|\overrightarrow{y^{\prime}}\right\|}=\frac{\|\vec{x}+\vec{y}\|}{\left\|\overrightarrow{x^{\prime}}+\overrightarrow{y^{\prime}}\right\|}=s .
$$

In particular, if $s=1$, we say that the Triangles $A B C$ and $E F G$ are congruent.
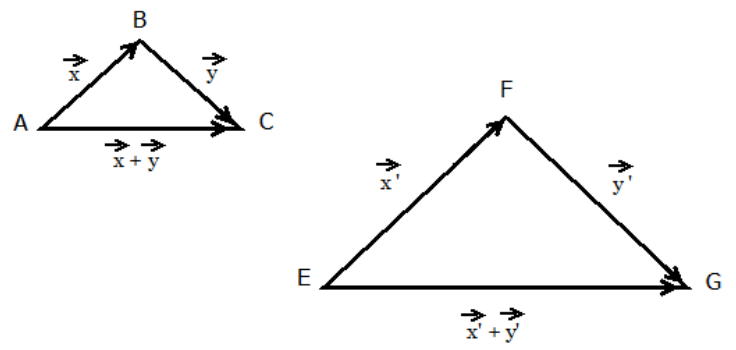

Figure 4.4: Similar Triangles in a Complex Hilbert Space

Theorem 4.2. (Side-Angle-Side (S.A.S.) Similarity in Complex Hilbert Spaces) Let $A B C$ and $E F G$ be two triangles in a complex Hilbert space $\mathbb{X}$, their sides given by the nonzero vectors $\vec{x}, \vec{y}, \vec{x}+\vec{y}$ and $\overrightarrow{x^{\prime}}, \overrightarrow{y^{\prime}}, \overrightarrow{x^{\prime}}+\overrightarrow{y^{\prime}}$, respectively as in Figure 4.4 . If

$$
\cos \theta(-\vec{x}, \vec{y})=\cos \theta^{\prime}\left(-\overrightarrow{x^{\prime}}, \overrightarrow{y^{\prime}}\right) \text { and } \frac{\|\vec{x}\|}{\left\|\overrightarrow{x^{\prime}}\right\|}=\frac{\|\vec{y}\|}{\left\|\overrightarrow{y^{\prime}}\right\|}
$$

then the triangles $E F G$ and $A B C$ are similar.

Proof : Let $A B C$ and $E F G$ be two triangles as illustrated in Figure 4.4. Since $\cos \theta(-\vec{x}, \vec{y})=\cos \theta^{\prime}\left(-\overrightarrow{x^{\prime}}, \overrightarrow{y^{\prime}}\right)$, we have

$$
\frac{\langle-\vec{x}, \vec{y}\rangle+\langle\vec{y},-\vec{x}\rangle}{2\|\vec{x}\|\|\vec{y}\|}=\frac{\left\langle-\overrightarrow{x^{\prime}}, \overrightarrow{y^{\prime}}\right\rangle+\left\langle\overrightarrow{y^{\prime}},-\overrightarrow{x^{\prime}}\right\rangle}{2\left\|\overrightarrow{x^{\prime}}\right\|\left\|\overrightarrow{y^{\prime}}\right\|} .
$$


Let

$$
\frac{\|\vec{x}\|}{\left\|\overrightarrow{x^{\prime}}\right\|}=\frac{\|\vec{y}\|}{\left\|\overrightarrow{y^{\prime}}\right\|}=s, \quad s>0
$$

It is enough to show that $\frac{\|\vec{x}+\vec{y}\|}{\left\|\overrightarrow{x^{\prime}}+\overrightarrow{y^{\prime}}\right\|}=s$. Equations (4.10) and (4.11) imply that

$$
\begin{aligned}
\|\vec{x}+\vec{y}\|^{2} & =\|\vec{x}\|^{2}+\|\vec{y}\|^{2}-2\|\vec{x}\|\|\vec{y}\| \frac{\langle-\vec{x}, \vec{y}\rangle+\langle\vec{y},-\vec{x}\rangle}{2\|\vec{x}\|\|\vec{y}\|} \\
& =s^{2}\left\|\overrightarrow{x^{\prime}}\right\|^{2}+s^{2}\left\|\overrightarrow{y^{\prime}}\right\|^{2}-2 s^{2}\left\|\overrightarrow{x^{\prime}}\right\|\left\|\overrightarrow{y^{\prime}}\right\| \frac{\left\langle-\overrightarrow{x^{\prime}}, \overrightarrow{y^{\prime}}\right\rangle+\left\langle\overrightarrow{y^{\prime}},-\overrightarrow{x^{\prime}}\right\rangle}{2\left\|\overrightarrow{x^{\prime}}\right\| \overrightarrow{y^{\prime}} \|} \\
& =s^{2}\left[\left\|\overrightarrow{x^{\prime}}\right\|^{2}+\left\|\overrightarrow{y^{\prime}}\right\|^{2}-\left\langle-\overrightarrow{x^{\prime}}, \overrightarrow{y^{\prime}}\right\rangle+\left\langle\overrightarrow{y^{\prime}},-\overrightarrow{x^{\prime}}\right\rangle\right] \\
& =s^{2}\left\langle\overrightarrow{x^{\prime}}+\overrightarrow{y^{\prime}}, \overrightarrow{x^{\prime}}+\overrightarrow{y^{\prime}}\right\rangle=s^{2}\left\|\overrightarrow{x^{\prime}}+\overrightarrow{y^{\prime}}\right\|^{2} .
\end{aligned}
$$
result.

Hence, $\frac{\|\vec{x}+\vec{y}\|^{2}}{\left\|\overrightarrow{x^{3}}+y^{\prime}\right\|^{2}}=s^{2}$, and by taking the positive square root we obtain the desired

Theorem 4.3. (Angle-Angle-Angle (A.A.A.) Similarity in Complex Hilbert Spaces) Let $A B C$ and $E F G$ be two triangles in a complex Hilbert space $\mathbb{X}$, their sides given by the nonzero vectors $\vec{x}, \vec{y}, \vec{x}+\vec{y}$ and $\overrightarrow{x^{\prime}}, \overrightarrow{y^{\prime}}, \overrightarrow{x^{\prime}}+\overrightarrow{y^{\prime}}$, respectively as in Figure 4.4. If

$$
\begin{gathered}
\sin \theta(\vec{x}+\vec{y}, \vec{x})=\sin \theta^{\prime}\left(\overrightarrow{x^{\prime}}+\overrightarrow{y^{\prime}}, \overrightarrow{x^{\prime}}\right) \quad \sin \theta(-\vec{x}, \vec{y})=\sin \theta^{\prime}\left(-\overrightarrow{x^{\prime}}, \overrightarrow{y^{\prime}}\right) \\
\sin \theta(-\vec{x}-\vec{y},-\vec{y})=\sin \theta^{\prime}\left(-\overrightarrow{x^{\prime}}-\overrightarrow{y^{\prime}},-\overrightarrow{y^{\prime}}\right),
\end{gathered}
$$

then Triangles EFG and $A B C$ are similar.

Proof : We want to show that

$$
\frac{\|\vec{y}\|}{\left\|\overrightarrow{y^{\prime}}\right\|}=\frac{\|\vec{x}\|}{\left\|\overrightarrow{x^{\prime}}\right\|}=\frac{\|\vec{x}+\vec{y}\|}{\left\|\overrightarrow{x^{\prime}}+\overrightarrow{y^{\prime}}\right\|}
$$

Now, by Theorem 4.1 we have

$$
\frac{\|\vec{y}\|}{\sin \theta(\vec{x}+\vec{y}, \vec{x})}=\frac{\|\vec{x}\|}{\sin \theta(-\vec{x}-\vec{y},-\vec{y})}=\frac{\|\vec{x}+\vec{y}\|}{\sin \theta(-\vec{x}, \vec{y})} .
$$

and

$$
\frac{\sin \theta\left(\overrightarrow{x^{\prime}}+\overrightarrow{y^{\prime}}, \overrightarrow{x^{\prime}}\right)}{\left\|\overrightarrow{y^{\prime}}\right\|}=\frac{\sin \theta\left(-\overrightarrow{x^{\prime}}-\overrightarrow{y^{\prime}},-\overrightarrow{y^{\prime}}\right)}{\left\|\overrightarrow{x^{\prime}}\right\|}=\frac{\sin \theta\left(-\overrightarrow{x^{\prime}}, \overrightarrow{y^{\prime}}\right)}{\left\|\overrightarrow{x^{\prime}}+\overrightarrow{y^{\prime}}\right\|}
$$


Equations (4.13) and (4.14) imply that

$$
\begin{aligned}
\frac{\|\vec{y}\| \sin \theta\left(\overrightarrow{x^{\prime}}+\overrightarrow{y^{\prime}}, \overrightarrow{x^{\prime}}\right)}{\left\|\overrightarrow{y^{\prime}}\right\| \sin \theta(\vec{x}+\vec{y}, \vec{x})}=\frac{\|\vec{x}\| \sin \theta\left(-\overrightarrow{x^{\prime}}-\overrightarrow{y^{\prime}},-\overrightarrow{y^{\prime}}\right)}{\left\|\overrightarrow{x^{\prime}}\right\| \sin \theta(-\vec{x}-\vec{y},-\vec{y})} \\
=\frac{\|\vec{x}+\vec{y}\| \sin \theta\left(-\overrightarrow{x^{\prime}}, \overrightarrow{y^{\prime}}\right)}{\left\|\overrightarrow{x^{\prime}}+\overrightarrow{y^{\prime}}\right\| \sin \theta(-\vec{x}, \vec{y})} .
\end{aligned}
$$

By applying Equations (4.12) to Equation (4.15), we obtain

$$
\frac{\|\vec{y}\|}{\left\|\overrightarrow{y^{\prime}}\right\|}=\frac{\|\vec{x}\|}{\left\|\overrightarrow{x^{\prime}}\right\|}=\frac{\|\vec{x}+\vec{y}\|}{\left\|\overrightarrow{x^{\prime}}+\overrightarrow{y^{\prime}}\right\|}
$$

as desired.

Corollary 4.1. Let $A B C$ and EFG be two triangles in a complex Hilbert space $\mathbb{X}$, their sides given by the nonzero vectors $\vec{x}, \vec{y}, \vec{x}+\vec{y}$ and $\overrightarrow{x^{\prime}}, \overrightarrow{y^{\prime}}, \overrightarrow{x^{\prime}}+\overrightarrow{y^{\prime}}$, respectively as in Figure 4.4. Assume that

$$
\frac{\|\vec{x}\|}{\left\|\overrightarrow{x^{\prime}}\right\|}=\frac{\|\vec{y}\|}{\left\|\overrightarrow{y^{\prime}}\right\|}=\frac{\|\vec{x}+\vec{y}\|}{\left\|\overrightarrow{x^{\prime}}+\overrightarrow{y^{\prime}}\right\|}=s, \quad s>0 .
$$

Then,

$$
\begin{aligned}
& \cos \theta(\vec{y},-\vec{x})=\cos \theta\left(\overrightarrow{y^{\prime}},-\overrightarrow{x^{\prime}}\right), \cos \theta(\vec{x}+\vec{y}, \vec{x})=\cos \theta\left(\overrightarrow{x^{\prime}}+\overrightarrow{y^{\prime}}, \overrightarrow{x^{\prime}}\right), \\
& \cos \theta(-\vec{x}-\vec{y},-\vec{y})=\cos \theta\left(-\overrightarrow{x^{\prime}}-\overrightarrow{y^{\prime}},-\overrightarrow{y^{\prime}}\right) .
\end{aligned}
$$

Proof: Since $\frac{\|\vec{x}\|}{\left\|\overrightarrow{x^{\prime}}\right\|}=\frac{\|\vec{y}\|}{\left\|\overrightarrow{y^{\prime}}\right\|}=\frac{\|\vec{x}+\vec{y}\|}{\left\|\overrightarrow{x^{\prime}}+\overrightarrow{y^{\prime}}\right\|}=s, s>0$ and

$$
\|\vec{x}+\vec{y}\|^{2}=\|\vec{x}\|^{2}+\|\vec{y}\|^{2}-[\langle\vec{y},-\vec{x}\rangle+\langle-\vec{x}, \vec{y}\rangle]
$$

it follows that

$$
s^{2}\left\|\overrightarrow{x^{\prime}}+\overrightarrow{y^{\prime}}\right\|^{2}=s^{2}\left\|\overrightarrow{x^{\prime}}\right\|^{2}+s^{2}\left\|\overrightarrow{y^{\prime}}\right\|^{2}-[\langle\vec{y},-\vec{x}\rangle+\langle-\vec{x}, \vec{y}\rangle] .
$$

Furthermore,

$$
s^{2}\left\|\overrightarrow{x^{\prime}}+\overrightarrow{y^{\prime}}\right\|^{2}=s^{2}\left\|\overrightarrow{x^{\prime}}\right\|^{2}+s^{2}\left\|\overrightarrow{y^{\prime}}\right\|^{2}-s^{2}\left[\left\langle\overrightarrow{y^{\prime}},-\overrightarrow{x^{\prime}}\right\rangle+\left\langle-\overrightarrow{x^{\prime}}, \overrightarrow{y^{\prime}}\right\rangle\right] .
$$

If we combine Equation (4.18) and Equation (4.19), we conclude that

$$
\langle\vec{y},-\vec{x}\rangle+\langle-\vec{x}, \vec{y}\rangle=s^{2}\left[\left\langle\overrightarrow{y^{\prime}},-\overrightarrow{x^{\prime}}\right\rangle+\left\langle-\overrightarrow{x^{\prime}}, \overrightarrow{y^{\prime}}\right\rangle\right]
$$


Since $\|\vec{x}\|\|\vec{y}\|=s^{2}\left\|\overrightarrow{x^{\prime}}\right\|\left\|\overrightarrow{y^{\prime}}\right\|$, Equation (4.20) is equivalent to

$$
\frac{\langle\vec{y},-\vec{x}\rangle+\langle-\vec{x}, \vec{y}\rangle}{\|\vec{x}\|\|\vec{y}\|}=\frac{s^{2}\left[\left\langle\overrightarrow{y^{\prime}},-\overrightarrow{x^{\prime}}\right\rangle+\left\langle-\overrightarrow{x^{\prime}}, \overrightarrow{y^{\prime}}\right\rangle\right]}{s^{2}\left\|\overrightarrow{x^{\prime}}\right\|\left\|\overrightarrow{y^{\prime}}\right\|}=\frac{\left\langle\overrightarrow{y^{\prime}},-\overrightarrow{x^{\prime}}\right\rangle+\left\langle-\overrightarrow{x^{\prime}}, \overrightarrow{y^{\prime}}\right\rangle}{\left\|\overrightarrow{x^{\prime}}\right\|\left\|\overrightarrow{y^{\prime}}\right\|} \text {. }
$$

Combining this identity with Definition 31 shows that

$$
\cos \theta(\vec{y},-\vec{x})=\cos \theta\left(\overrightarrow{y^{\prime}},-\overrightarrow{x^{\prime}}\right) .
$$

Similar arguments, which are left to the reader, show that

$$
\cos \theta(-\vec{x}-\vec{y},-\vec{y})=\cos \theta\left(-\overrightarrow{x^{\prime}}-\overrightarrow{y^{\prime}},-\overrightarrow{y^{\prime}}\right)
$$

and

$$
\cos \theta(\vec{x}+\vec{y}, \vec{x})=\cos \theta\left(\overrightarrow{x^{\prime}}+\overrightarrow{y^{\prime}}, \overrightarrow{x^{\prime}}\right)
$$

\subsection{Addition Formulas in Complex Hilbert Spaces}

In this section we develop addition formulas for the cosine and sine functions of a complex Hilbert space as defined in Definitions 31 and 32. But in order to do so, we need the following two propositions.

Proposition 4.3. Let $A B C$ be a triangle in a complex Hilbert space whose sides are the nonzero vectors $\vec{x}, \vec{y}$, and $\vec{x}+\vec{y}$ with $\langle-\vec{x}, \vec{y}\rangle+\langle\vec{y},-\vec{x}\rangle=0$. See Figure 4.5.Then

$$
\cos \theta(-\vec{x}, \vec{y})=0 \quad \sin \theta(-\vec{x}, \vec{y})=1
$$

and

$\cos \theta(\vec{x}+\vec{y}, \vec{x})=\sin \theta(-\vec{x}-\vec{y},-\vec{y}) \quad \sin \theta(\vec{x}+\vec{y}, \vec{x})=\cos \theta(-\vec{x}-\vec{y},-\vec{y})$.

Proof: Let $A B C$ be the triangle illustrated in Figure 4.5.

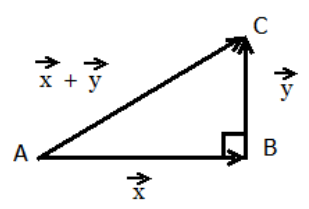

Figure 4.5: $\cos \theta=\sin \left(\frac{\pi}{2}-\theta\right)$ 
First, we prove Equation (4.21). Indeed, since $\langle-\vec{x}, \vec{y}\rangle+\langle\vec{y},-\vec{x}\rangle=0$, Definition 31 implies that

$$
\cos \theta(-\vec{x}, \vec{y})=\frac{\langle-\vec{x}, \vec{y}\rangle+\langle\vec{y},-\vec{x}\rangle}{2\|\vec{x}\|\|\vec{y}\|}=0 .
$$

Then, combining Equation (4.23) with Definition 32 shows that $\sin \theta(-\vec{x}, \vec{y})=1$.

To show the first identity of (4.22) is true, notice that by Theorem 4.1

$$
\frac{\sin \theta(-\vec{x}-\vec{y},-\vec{y})}{\|\vec{x}\|}=\frac{\sin \theta(-\vec{x}, \vec{y})}{\|\vec{x}+\vec{y}\|} .
$$

Since $\sin \theta(-\vec{x}, \vec{y})=1$, it follows that Equation (4.24) can be written as

$$
\sin \theta(-\vec{x}-\vec{y},-\vec{y})=\frac{\|\vec{x}\|}{\|\vec{x}+\vec{y}\|} .
$$

On the other hand, since $\langle-\vec{x}, \vec{y}\rangle+\langle\vec{y},-\vec{x}\rangle=0=\langle\vec{x}, \vec{y}\rangle+\langle\vec{y}, \vec{x}\rangle$, Definition 31 becomes

$$
\begin{aligned}
\cos \theta(\vec{x}+\vec{y}, \vec{x}) & =\frac{\langle\vec{x}+\vec{y}, \vec{x}\rangle+\langle\vec{x}, \vec{x}+\vec{y}\rangle}{2\|\vec{x}\|\|\vec{x}+\vec{y}\|} \\
& =\frac{\langle\vec{x}, \vec{x}\rangle+\langle\vec{y}, \vec{x}\rangle+\langle\vec{x}, \vec{x}\rangle+\langle\vec{x}, \vec{y}\rangle}{2\|\vec{x}\|\|\vec{x}+\vec{y}\|} \\
& =\frac{\|\vec{x}\|}{\|\vec{x}+\vec{y}\|} .
\end{aligned}
$$

Combining Equations (4.25) and (4.26) completes the proof. A similar argument left to the reader proves the second identity of (4.22).

Proposition 4.4. Let $\vec{x}$ and $\vec{y}$ be two nonzero linearly independent vectors in a complex Hilbert space $\mathbb{X}$ with $\langle\vec{x}, \vec{y}\rangle+\langle\vec{y}, \vec{x}\rangle \neq 0$. Then

$$
\frac{1}{\cos ^{2} \theta(\vec{x}, \vec{y})}=\frac{\sin ^{2} \theta(\vec{x}, \vec{y})}{\cos ^{2} \theta(\vec{x}, \vec{y})}+1 .
$$

Proof : Definition 32 implies that

$$
\sin ^{2} \theta(\vec{x}, \vec{y})+\cos ^{2} \theta(\vec{x}, \vec{y})=1
$$

Since $\langle\vec{x}, \vec{y}\rangle+\langle\vec{y}, \vec{x}\rangle \neq 0$, and $\cos \theta(\vec{x}, \vec{y})=\frac{\langle\vec{x}, \vec{y}\rangle+\langle\vec{y}, \vec{x}\rangle}{2\|\vec{x}\|\|\vec{y}\|}$, we deduce that $\cos \theta(\vec{x}, \vec{y}) \neq 0$. Therefore, Equation (4.28) is equivalent to Equation (4.27). 
We are now in a position to prove the trigonometric addition formulas for a complex Hilbert space.

Theorem 4.4. Let $\vec{x}, \vec{y}$, and $\vec{z}$ be three nonzero vectors as in a complex Hilbert space $\mathbb{X}$ as illustrated in Figure 4.6, such that $\|\vec{y}\|=1, \cos \theta(\vec{x}, \vec{y})>0$ and $\cos \theta(\vec{y}, \vec{z})>0$. Then,

$$
\begin{aligned}
\cos [\angle \theta(\vec{x}, \vec{y})+\angle \theta(\vec{y}, \vec{z})] & =\cos \theta(\vec{x}, \vec{y}) \cos \theta(\vec{y}, \vec{z}) \\
& -\sin \theta(\vec{x}, \vec{y}) \sin \theta(\vec{y}, \vec{z}),
\end{aligned}
$$

and

$$
\begin{aligned}
\sin [\angle \theta(\vec{x}, \vec{y})+\angle \theta(\vec{y}, \vec{z})] & =\sin \theta(\vec{x}, \vec{y}) \cos \theta(\vec{y}, \vec{z}) \\
& +\sin \theta(\vec{y}, \vec{z}) \cos \theta(\vec{x}, \vec{y}) .
\end{aligned}
$$

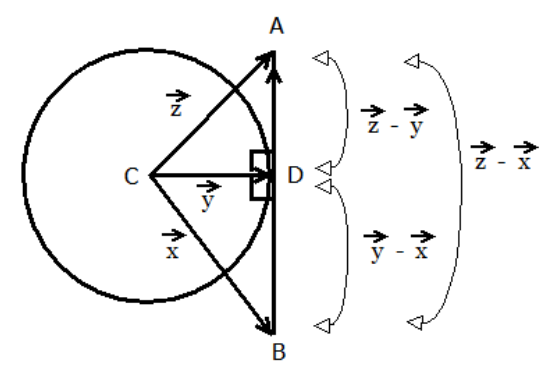

Figure 4.6: The Formulas of Cosine and Sine

Proof : Applying Theorem 4.1 to Triangle $D B C$ shows that

$$
\sin \theta(\vec{y}-\vec{x},-\vec{x})=\frac{\sin \theta(\vec{y}-\vec{x},-\vec{x})}{\|\vec{y}\|}=\frac{\sin \theta(\vec{x}, \vec{y})}{\|\vec{y}-\vec{x}\|}, \quad \text { since }\|\vec{y}\|=1 .
$$

Also by (4.22) of Proposition 4.3 we have $\cos \theta(\vec{x}, \vec{y})=\sin \theta(\vec{y}-\vec{x},-\vec{x})$. Thus, Equation (4.31) simplifies to

$$
\|\vec{y}-\vec{x}\|=\frac{\sin \theta(\vec{x}, \vec{y})}{\cos \theta(\vec{x}, \vec{y})}
$$

Similarly, in Triangle $D B C$, we apply Theorem 4.1 to obtain

$$
\frac{\sin \theta(\vec{x}-\vec{y},-\vec{y})}{\|\vec{x}\|}=\frac{\sin \theta(\vec{y}-\vec{x},-\vec{x})}{\|\vec{y}\|}=\sin \theta(\vec{y}-\vec{x},-\vec{x}) .
$$


Since Proposition 4.3 implies that $1=\sin \theta(\vec{x}-\vec{y},-\vec{y})$, by once again applying (4.22), we see that Equation (4.33) becomes

$$
\|\vec{x}\|=\frac{1}{\cos \theta(\vec{x}, \vec{y})}
$$

The corresponding formulas for Triangle $A C D$ are obtained by replacing $\vec{x}$ with $\vec{z}$ in Equations (4.32) and (4.34), namely

$$
\|\vec{y}-\vec{z}\|=\frac{\sin \theta(\vec{y}, \vec{z})}{\cos \theta(\vec{y}, \vec{z})}
$$

and

$$
\|\vec{z}\|=\frac{1}{\cos \theta(\vec{y}, \vec{z})}
$$

By construction (see Figure 4.6), we have

$$
\begin{aligned}
\|\vec{z}-\vec{x}\|^{2} & =(\|\vec{z}-\vec{y}\|+\|\vec{y}-\vec{x}\|)^{2} \\
& =\|\vec{z}-\vec{y}\|^{2}+2\|\vec{z}-\vec{y}\|\|\vec{y}-\vec{x}\|+\|\vec{y}-\vec{x}\|^{2} .
\end{aligned}
$$

Substitute Equations (4.35) and (4.32) into Equation (4.37) to obtain

$$
\|\vec{z}-\vec{x}\|^{2}=\frac{\sin ^{2} \theta(\vec{z}, \vec{y})}{\cos ^{2} \theta(\vec{z}, \vec{y})}+2 \frac{\sin \theta(\vec{z}, \vec{y}) \sin \theta(\vec{x}, \vec{y})}{\cos \theta(\vec{z}, \vec{y}) \cos \theta(\vec{x}, \vec{y})}+\frac{\sin ^{2} \theta(\vec{x}, \vec{y})}{\cos ^{2} \theta(\vec{x}, \vec{y})}
$$

Now the cosine theorem applied to Triangle $A B C$ shows that

$$
\|\vec{z}-\vec{x}\|^{2}=\|\vec{x}\|^{2}+\|\vec{z}\|^{2}-2\|\vec{x}\|\|\vec{z}\| \cos [\angle \theta(\vec{x}, \vec{y})+\angle \theta(\vec{y}, \vec{z})] .
$$

Substitute Equations (4.34) and (4.36) into (4.39) to obtain

$$
\|\vec{z}-\vec{x}\|^{2}=\frac{1}{\cos ^{2} \theta(\vec{x}, \vec{y})}+\frac{1}{\cos ^{2} \theta(\vec{z}, \vec{y})}-2 \frac{\cos [\angle \theta(\vec{x}, \vec{y})+\angle \theta(\vec{y}, \vec{z})]}{\cos \theta(\vec{x}, \vec{y}) \cos \theta(\vec{z}, \vec{y})}
$$

Then, apply Proposition 4.4 to (4.40) and obtain

$$
\|\vec{z}-\vec{x}\|^{2}=2+\frac{\sin ^{2} \theta(\vec{x}, \vec{y})}{\cos ^{2} \theta(\vec{x}, \vec{y})}+\frac{\sin ^{2} \theta(\vec{y}, \vec{z})}{\cos ^{2} \theta(\vec{y}, \vec{z})}-2 \frac{\cos [\angle \theta(\vec{x}, \vec{y})+\angle \theta(\vec{y}, \vec{z})]}{\cos \theta(\vec{x}, \vec{y}) \cos \theta(\vec{z}, \vec{y})} .
$$

Set Equation (4.38) equal to Equation (4.41) to obtain

$$
\frac{\sin \theta(\vec{y}, \vec{z}) \sin \theta(\vec{x}, \vec{y})}{\cos \theta(\vec{y}, \vec{z}) \cos \theta(\vec{x}, \vec{y})}=1-\frac{\cos [\angle \theta(\vec{x}, \vec{y})+\angle \theta(\vec{y}, \vec{z})]}{\cos \theta(\vec{x}, \vec{y}) \cos \theta(\vec{y}, \vec{z})}
$$


Now if we multiply both sides of (4.42) by $-\cos \theta(\vec{x}, \vec{y}) \cos \theta(\vec{y}, \vec{z})$, we obtain Identity (4.29).

Now, we verify Identity (4.30). First add Equations (4.32) and (4.35) to obtain

$$
\|\vec{y}-\vec{x}\|+\|\vec{y}-\vec{z}\|=\frac{\sin \theta(\vec{x}, \vec{y}) \cos \theta(\vec{y}, \vec{z})+\sin \theta(\vec{y}, \vec{z}) \cos \theta(\vec{x}, \vec{y})}{\cos \theta(\vec{x}, \vec{y}) \cos \theta(\vec{y}, \vec{z})}
$$

Next, observe that Proposition 4.3 says

$$
\cos \theta(\vec{y}, \vec{z})=\sin \theta(\vec{y}-\vec{z},-\vec{z})=\sin \theta(\vec{x}-\vec{z},-\vec{z}) .
$$

Combine Equations (4.44) and (4.34) to obtain

$$
\cos \theta(\vec{x}, \vec{y}) \cos \theta(\vec{y}, \vec{z})=\frac{\sin \theta(\vec{x}-\vec{z},-\vec{z})}{\|\vec{x}\|}
$$

Now, the sine theorem applied to Triangle $A B C$ gives

$$
\frac{\sin [\angle \theta(\vec{x}, \vec{y})+\angle \theta(\vec{y}, \vec{z})]}{\|\vec{y}-\vec{x}\|+\|\vec{y}-\vec{z}\|}=\frac{\sin \theta(\vec{x}-\vec{z},-\vec{z})}{\|\vec{x}\|}
$$

Combine Equations (4.45) and (4.46) to obtain

$$
\frac{\sin [\angle \theta(\vec{x}, \vec{y})+\angle \theta(\vec{y}, \vec{z})]}{\|\vec{y}-\vec{x}\|+\|\vec{y}-\vec{z}\|}=\cos \theta(\vec{x}, \vec{y}) \cos \theta(\vec{y}, \vec{z}) .
$$

Then, if we substitute (4.43) into (4.46) and simplify, we obtain

$$
\frac{\sin [\angle \theta(\vec{x}, \vec{y})+\angle \theta(\vec{y}, \vec{z})]}{\sin \theta(\vec{x}, \vec{y}) \cos \theta(\vec{y}, \vec{z})+\sin \theta(\vec{y}, \vec{z}) \cos \theta(\vec{x}, \vec{y})}=1
$$

an identity equivalent to Identity (4.30).

Observe that the left sides of Equations (4.29) and (4.30) are defined only for angles $\angle \theta(\vec{x}, \vec{y})$ and $\angle \theta(\vec{y}, \vec{z})$ whose cosines are nonnegative. However, the right sides of Equations (4.29) and (4.30) are well defined (via Definition 31 and Definition 32) for $\angle \theta(\vec{x}, \vec{y})$ and $\angle \theta(\vec{y}, \vec{z})$ with negative, zero, and positive values for cosine. Hence, we define the left sides of Equations (4.29) and (4.30) for all angles $\angle \theta(\vec{x}, \vec{y})$ and $\angle \theta(\vec{y}, \vec{z})$ by the corresponding well defined right hand sides.

We end this chapter by applying above defined extensions of Equations (4.29) and (4.30) to show that the cosine of the sum of the interior angles of any triangle in a complex Hilbert space is $\pi$. 
Theorem 4.5. Let $A B C$ be a triangle in a complex Hilbert space $\mathbb{X}$ whose sides are the nonzero vectors $\vec{x}, \vec{y}$, and $\vec{x}-\vec{y}$; see Figure 4 .1. Let

$$
\alpha:=\angle \theta(\vec{x}, \vec{y}) \quad \beta:=\angle \theta(\vec{x}-\vec{y}, \vec{x}), \quad \gamma:=\angle \theta(\vec{y}, \vec{y}-\vec{x}) .
$$

Then $\cos (\alpha+\beta+\gamma)=-1$.

Proof : Let

$$
\begin{gathered}
a:=\langle\vec{x}, \vec{y}\rangle+\langle\vec{y}, \vec{x}\rangle \quad b:=\langle\vec{x}, \vec{x}-\vec{y}\rangle+\langle\vec{x}-\vec{y}, \vec{x}\rangle \\
c:=\langle\vec{y}, \vec{y}-\vec{x}\rangle+\langle\vec{y}-\vec{x}, \vec{y}\rangle .
\end{gathered}
$$

Then,

$\cos \alpha=\frac{a}{2\|\vec{x}\|\|\vec{y}\|} \quad \cos \beta=\frac{b}{2\|\vec{x}\|\|\vec{x}-\vec{y}\|} \quad \cos \gamma=\frac{c}{2\|\vec{y}\|\|\vec{x}-\vec{y}\|}$, and

$$
\begin{gathered}
\sin \alpha=\sqrt{1-\frac{a^{2}}{4\|\vec{x}\|^{2}\|\vec{y}\|^{2}}} \quad \sin \beta=\sqrt{1-\frac{b^{2}}{4\|\vec{x}\|^{2}\|\vec{x}-\vec{y}\|^{2}}} \\
\sin \gamma=\sqrt{1-\frac{c^{2}}{4\|\vec{y}\|^{2}\|\vec{x}-\vec{y}\|^{2}}}
\end{gathered}
$$

Now by (4.29) we have

$$
\cos ((\alpha+\beta)+\gamma)=\cos (\alpha+\beta) \cos \gamma-\sin (\alpha+\beta) \sin \gamma
$$

By applying Equations (4.29) and (4.30) to Equation (4.48), we rewrite Equation (4.48) as

$$
\cos ((\alpha+\beta)+\gamma)=T_{1}-T_{2}-T_{3}-T_{4}
$$

where

$$
\begin{array}{ll}
T_{1}:=\cos \alpha \cos \beta \cos \gamma & T_{2}:=\sin \alpha \sin \beta \cos \gamma \\
T_{3}:=\sin \alpha \cos \beta \sin \gamma & T_{4}=\cos \alpha \sin \beta \sin \gamma
\end{array}
$$

Notice that

$$
\|\vec{x}-\vec{y}\|^{2}=\|\vec{x}\|^{2}+\|\vec{y}\|^{2}-a \quad c=2\|\vec{y}\|^{2}-a \quad b=2\|\vec{x}\|^{2}-a .
$$

The identities at (4.49) imply that

$$
a+b=2\|\vec{x}\|^{2} \quad \text { and } \quad b+c=2\|\vec{x}\|^{2}+2\|\vec{y}\|^{2}-2 a .
$$


Then,

$$
\begin{aligned}
T_{1} & =\frac{a b c}{8\|\vec{x}\|^{2}\|\vec{y}\|^{2}\|\vec{x}-\vec{y}\|^{2}}=\frac{a\left(2\|\vec{x}\|^{2}-a\right)\left(2\|\vec{y}\|^{2}-a\right)}{8\|\vec{x}\|^{2}\|\vec{y}\|^{2}\|\vec{x}-\vec{y}\|^{2}} \\
& =\frac{a\left(4\|\vec{x}\|^{2}\|\vec{y}\|^{2}-2 a\|\vec{x}\|^{2}-2 a\|\vec{y}\|^{2}+a^{2}\right)}{8\|\vec{x}\|^{2}\|\vec{y}\|^{2}\|\vec{x}-\vec{y}\|^{2}}
\end{aligned}
$$

To calculate $T_{2}$, we need to first calculate an expression for $\sin \alpha \sin \beta$. Use the identities of (4.49) to obtain

$$
\begin{aligned}
\sin \alpha \sin \beta & =\sqrt{1-\frac{a^{2}}{4\|\vec{x}\|^{2}\|\vec{y}\|^{2}}} \sqrt{1-\frac{b^{2}}{4\|\vec{x}\|^{2}\|\vec{x}-\vec{y}\|^{2}}} \\
& =\sqrt{\frac{\left.4\|\vec{x}\|\right|^{2}\|\vec{y}\|^{2}-a^{2}}{4\|\vec{x}\|^{2}\|\vec{y}\|^{2}}} \sqrt{1-\frac{\left(2\|\vec{x}\|^{2}-a\right)^{2}}{4\|\vec{x}\|^{2}\left(\|\vec{x}\|^{2}+\|\vec{y}\|^{2}-a\right)}} \\
& =\sqrt{\frac{\left(4\|\vec{x}\|^{2}\|\vec{y}\|^{2}-a^{2}\right)^{2}}{16\|\vec{x}\|^{4}\|\vec{y}\|^{2}\|\vec{x}-\vec{y}\|^{2}}} .
\end{aligned}
$$

Applying the Cauchy-Schwarz inequality to the previous line shows that

$$
\sin \alpha \sin \beta=\frac{4\|\vec{x}\|^{2}\|\vec{y}\|^{2}-a^{2}}{4\|\vec{x}\|^{2}\|\vec{y}\|\|\vec{x}-\vec{y}\|} .
$$

Thus, by (4.53) we have

$$
T_{2}=\frac{\left(4\|\vec{x}\|^{2}\|\vec{y}\|^{2}-a^{2}\right)\left(2\|\vec{y}\|^{2}-a\right)}{8\|\vec{x}\|\left\|^{2}\right\| \vec{y}\left\|^{2}\right\| \vec{x}-\vec{y} \|^{2}}=\frac{\left(4\|\vec{x}\|\left\|^{2}\right\| \vec{y} \|^{2}-a^{2}\right) c}{8\|\vec{x}\|^{2}\|\vec{y}\|^{2}\|\vec{x}-\vec{y}\|^{2}} .
$$

Calculations similar to the ones used obtain Identity (4.54) show that

$$
T_{3}=\frac{\left(4\|\vec{x}\|^{2}\|\vec{y}\|^{2}-a^{2}\right)\left(2\|\vec{x}\|^{2}-a\right)}{8\|\vec{x}\|\left\|^{2}\right\| \vec{y}\left\|^{2}\right\| \vec{x}-\vec{y} \|^{2}}=\frac{\left(4\|\vec{x}\|\left\|^{2}\right\| \vec{y} \|^{2}-a^{2}\right) b}{8\|\vec{x}\|^{2}\|\vec{y}\|^{2}\|\vec{x}-\vec{y}\|^{2}} .
$$

and that

$$
T_{4}=\frac{a\left(4\|\vec{x}\|^{2}\|\vec{y}\|^{2}-a^{2}\right)}{8\|\vec{x}\|^{2}\|\vec{y}\|^{2}\|\vec{x}-\vec{y}\|^{2}} .
$$

It follows by Equations (4.51), (4.54), (4.55), and (4.56) that

$$
\begin{aligned}
& T_{1}-T_{2}-T_{3}-T_{4}=\frac{a b c-\left(4\|\vec{x}\|^{2}\|\vec{y}\|^{2}-a^{2}\right)(a+b+c)}{8\|\vec{x}\|^{2}\|\vec{y}\|^{2}\|\vec{x}-\vec{y}\|^{2}} \\
& =\frac{-8\|\vec{x}\|\left\|^{4}\right\| \vec{y}\left\|^{2}-8\right\| \vec{x}\left\|^{2}\right\| \vec{y}\left\|^{4}+8\right\| \vec{x}\left\|^{2}\right\| \vec{y} \|^{2} a}{8\|\vec{x}\|^{2}\|\vec{y}\|^{2}\left(\|\vec{x}\|^{2}+\|\vec{y}\|^{2}-a\right)}=-1 .
\end{aligned}
$$


Remark 4.1. Let $\vec{x}$ and $\vec{y}$ be two vectors in a complex Hilbert space. At the beginning of Section 4.2 , we defined $\angle \theta(\vec{x}, \vec{y})$ to be the convex subset of $\mathbb{P}$ between the rays $l_{x}$ and $l_{y}$, where $\mathbb{P}$ is the plane determined by $\vec{x}$ and $\vec{y}$. The complementary set $P-\angle \theta(\vec{x}, \vec{y})$ is the complementary angle $\angle \cos (2 \pi-\theta)(\vec{x}, \vec{y})$. We define

$$
\begin{aligned}
\cos (2 \pi-\theta)(\vec{x}, \vec{y}) & :=\cos \theta(\vec{x}, \vec{y}) \\
\sin (2 \pi-\theta)(\vec{x}, \vec{y}) & :=-\sin \theta(\vec{x}, \vec{y}) .
\end{aligned}
$$

The definitions of (4.58) are then consistent with the additions formulas of Theorem 4.4 if we define

$$
\begin{aligned}
\cos (-\theta)(\vec{x}, \vec{y}) & :=\cos \theta(\vec{x}, \vec{y}) \\
\sin (-\theta)(\vec{x}, \vec{y}) & :=-\sin \theta(\vec{x}, \vec{y}) .
\end{aligned}
$$




\section{Chapter 5}

\section{Appendices}

\section{.1 Appendix A: Euclid's Axioms}

1. To draw a line through two points.

2. To extend a given line.

3. To draw a circle with given center through a given point.

4. All right angles angles are equal.

5. If a line crossing two other lines makes the interior angles on the same side less than two right angles, then these two lines will meet on the side when extended for enough.

\section{.2 Appendix B: Euclid's Common Notions}

1. Things equal to the same thing are equal.

2. Equals added to equals are equal.

3. Equals subtracted from equals are equal.

4. Things which coincide are equal.

5. The whole is greater than the part. 


\section{.3 Appendix C: Hilbert's Axioms of Plane Geometry}

\section{Axioms of Incidence:}

I1. For every two distinct points $A$ and $B$ there exists a unique line $\ell$ that contains each of the points $\mathrm{A}$ and $\mathrm{B}$.

I2. There exists at least two points on a line.

I3. There exist at least three points that do not lie on a line.

\section{Axioms of Betweeness:}

B1. If a point $\mathrm{B}$ lies between a point $A$ and a point $C$, then the points $A, B$, and $C$ are three distinct points of a line, and $B$ then also lies between $C$ and $A$.

B2. For two points $A$ and $C$, there always exists at least one point $B$ on the line $l_{A C}$ such that $C$ lies between $A$ and $B$.

B3. Of any three points on a line $\ell$ there exists no more than one point that lies between the other two.

B4. Let $A, B$ and $C$ be three noncollinear points and let $\ell$ be a line not containing any of $A, B$, and $C$. If $\ell$ contains a point $D$ lying between $A$ and $B$, then it must also contain either a point between $A$ and $C$ or a point between $B$ and $C$.

\section{Axioms of Congruence:}

C1. Given a line segment $\overline{A B}$, and given a ray $\overrightarrow{C P}_{\infty}$ originating at a point $C$, there exists a unique point $D$ on the ray $\overrightarrow{C P}_{\infty}$ such that $\overline{A B} \cong \overline{C D}$.

C2. If $\overline{A B} \cong \overline{C D}$ and $\overline{C D} \cong \overline{E F}$, then $\overline{A B} \cong \overline{E F}$. Every line segment is congruent to itself.

C3. On the line $\ell$ let $\overline{A B}$ and $\overline{B C}$ be two line segments which except for $B$ have no point in common. Furthermore, on the same or on another line $\ell^{\prime}$ let $\overline{A^{\prime} B^{\prime}}$ and $\overline{B^{\prime} C^{\prime}}$ be two line segments which except for $B^{\prime}$ have no point in common. In the case, if $\overline{A B} \cong \overline{A^{\prime} B^{\prime}}$ and $\overline{B C} \cong \overline{B^{\prime} C^{\prime}}$, then $\overline{A C} \cong \overline{A^{\prime} C^{\prime}}$.

C4. Given an angle $\angle B A C$ and given a ray $\overrightarrow{D F}_{\infty}$, there exists a unique ray $\overrightarrow{D E}_{\infty}$ on a given side of the line $l_{D F}$, such that $\angle B A C \cong \angle E D F$.

C5. For any three angles $\angle B A C, \angle E D F, \angle L M N$, if $\angle B A C \cong \angle E D F$ and $\angle B A C \cong \angle L M N$, then $\angle L M N \cong \angle E D F$. Every angle is congruent to itself.

C6. (Side Angle Side) Given triangles $\triangle A B C, \triangle D E F$, suppose that $A B \cong$ $D E$, and $A C \cong D F$, and $\angle B A C \cong \angle E D F$, Then, the two triangles are congruent, namely, $B C \cong \angle E F, \angle A B C \cong \angle D E F$, and $\angle A C B \cong \angle D F E$. 
4. Axiom of Parallel: (P) For each line $\ell$ and each point $A$ then there is at most one line containing $A$ and parallel to $\ell$.

5. Axiom of Circle-Circle-Intersection Property: (E) Given two circles $\Gamma_{1}, \Gamma_{2}$. If $\Gamma_{1}$ contains at least one point inside $\Gamma_{2}$, and $\Gamma_{1}$ contains at least one point outside $\Gamma_{2}$, the two circles meet at two points.

We consider the definition of Euclidean Plane that Hartshorne uses, see [20] on page 112.

Definition of A Euclidean Plane. A Euclidean Plane is a set of points with subsets called lines, and undefined notions of betweenness and congruence satisfying the axioms I1-I3, B1-B4, C1-C6, P and E that are stated above.

\section{.4 Appendix D: Axioms of Vector Spaces and Axioms of The Real Numbers}

A vector space $V$ over a field $\mathbf{K}$ is a nonempty set of elements $v, u, \ldots$ (called vectors) together with two algebraic operations. These operations are called vector addition and multiplication of vectors by scalars, that is, by elements of $\mathbf{K}$ such that the two operations satisfy the following ten axioms.

1. Vector addition associates with each ordered pair $(u, v)$ of vectors a vector $(u+v)$ in such a way the four following properties hold.

2. Commutative $u+v=v+u$ for all $u, v \in V$.

3. Associativity. $(u+v)+w=u+(v+w)$ for all $u, v, w \in V$.

4. Additive identity. There exists an element $\mathbf{0} \in V$ (the zero vector) such that $v+\mathbf{0}=\mathbf{0}$ for all $v \in V$.

5. Additive inverse For every $v \in V$, there exists $w \in V$ such that $v+w=\mathbf{0}$.

6. Scalar multiplication associates with every vector $v$ and scalar $t$ a vector $t v$ in such a way the four following properties hold.

7. Multiplicative identity. $1 v=v$ for all $v \in V$.

8. Associativity. $(s t) v=s(t v)$ for all $v \in V$ and all $s, t \in \mathbf{K}$.

9. Distributive property. $s(u+v)=s u+s v$ for all $u, v \in V$ and all $s \in \mathbf{K}$.

10. Distributive property. $(s+t) v=s v+t v$ for all $v \in V$ and all $s, t \in \mathbf{K}$. 
The Axioms of The Real Numbers

Algebraic Axioms: for each $a, b, c \in \mathbb{R}$

1. $(a+b)+c=a+(b+c)$ (the associative law for addition),

2. $a+b=b+a$ (the commutative law for addition),

3. there exists $0 \in \mathbb{R}$ such that for each $a \in \mathbb{R}, a+0=a$,

4. for each $a \in \mathbb{R}$ there exists an element $-a \in \mathbb{R}$ such that $a+(-a)=0$,

5. $a(b c)=(a b) c$ (the associative law for multiplication),

6. $a b=b a$ (the commutative law for multiplication),

7. there exists $1 \in \mathbb{R}$ such that for each $a \in \mathbb{R}, a 1=a$,

8. for all $a \neq 0 \in \mathbb{R}$, there exists $\frac{1}{a} \in \mathbb{R}$ such that $a\left(\frac{1}{a}\right)=1$ (the law of reciprocal), and

9. $a(b+c)=a b+a c$ (the distributive law).

\section{The Axioms of Order:}

1. $a<b$ and $b<c$ implies that $a<c$ (the transitive law);

2. for all $a, b \in \mathbb{R}$ exactly one pf the following holds: $a<b$, or $a>b$, or $a=b$;

3. for all $a, b, c \in \mathbb{R}, a<b$ implies $a+c<b+c$ (the law of compatibility with addition);

4. for all $a, b, c \in \mathbb{R}$, if $a<b$ and $c>0$, then $a c<b c$ (the law of compatibility with multiplication).

The algebraic axioms and the axioms of order stated above make the set of real numbers into what is called an ordered field. In addition, this ordered field satisfies the two following axioms.

The Axiom of Archimedes: for all $a>0$ in $\mathbb{R}$ there exists $n \in \mathbb{N}$ such that $n>a$.

The Axiom of Completeness: every non-empty subset of $\mathbb{R}$ that is bounded above has a least upper bound in $\mathbb{R}$. 


\section{Bibliography}

[1] H. Albahboh ; H. Gingold ; J. Quaintance, Trigonometry in complex inner product spaces. Linear Algebra Appl. 575 (2019), 216-234

[2] H. Anton, Elementary Linear Algebra. 9. ed. J. Wiley, 2005.

[3] T. M. Apostol, Calculus. 2nd ed. Waltham, Mass. Blaisdell Pub., 1967.

[4] S. Axler, Linear Algebra Done Right. Third Edition. Springer International Publishing, 2015.

[5] V. Balestro, A. G. Horváth, H. Martini, and R. Teixeira, Angles in Normed Spaces, Aequationes Math., 91(2017), No. 2, 201-236.

[6] V. Balestro, A. G. Horváth, and H. Martini, Angle measures, general rotations, and roulettes in normed planes, Analysis and Mathematical Physics, 7(2017), No. 4, 549-575.

[7] Carl M. Bender, Dorje C. Brody, and Hugh F. Jones, Complex Extension of Quantum Mechanics, arXiv:quant-ph/0208076v2 30 Oct2002.

[8] D. D. Berkey, Calculus. 2nd ed. New York. Saunders College Pub., 1988.

[9] L. Bers, F. Karal, Calculus. 2nd ed. New York. Holt, Rinehart and Winston, 1976.

[10] W. E. Boyce, R.C. Diprima, and D.B. Meade, Elementary Differential Equations and Boundary Value Problems, 11th edition, John Wiley and Sons, Inc., 2017.

[11] F. Brauer\& A Nohel, The Qualitative Theory of Ordinary Differential Equations: An Introduction. Dover Publications [Paperback], Mineola NY, 1989. Reprinted from W. A. Benjamin, Inc. New York, 1969.

[12] R. C. Buck, E. F. Buck, Advanced Calculus. 2nd ed. New York. McGraw-Hill 1965. 
[13] M. J. Crowe, A History of Vector Analysis: The Evolution of the Idea of a Vectorial System. Notre Dame. University of Notre Dame Press, 1967.

[14] K. O. Friedrichs, From Pythagoras to Einstein, The Mathematical Association of America, 1965.

[15] A. Galantai and Cs.J. Hegedus, Jordan's Principal Angles In Complex Vector Spaces, Numerical Linear Algebra With Applications, 13 (2006), 589-598.

[16] J. Gallier, J. Quaintance, Algebra, Topology, Differential Calculus and Optimization Theory For Computer Science and Engineering, 2019.

[17] J. Gallier, J. Quaintance, Aspects of Convex Geometry Polyhedra, Linear Programming, Shellings, Voronoi Diagrams, Delaunay Triangulations 2017.

[18] F. Gallone, Hilbert Space and Quantum Mechanics, World Scientific, Singapore, 2015.

[19] H. Gingold; Y. Gingold; S. Hamad, A spherical projection of a complex Hilbert space is conformal iff it is the stereographic projection. Differ. Geom. Dyn. Syst. 20 (2018), 38-70.

[20] R. Hartshorne, Geometry: Euclid and Beyond, Springer, New York, 2000.

[21] Jordan \& P. Smith, Nonlinear Ordinary Differential Equations: An Introduction for Scientists and Engineers, 3rd edition, Oxford Texts in Applied and Engineering Mathematics, 2007.

[22] M. Obst, A perimeter-based angle measure in Minkowski planes, Aequationes Math., 12(2018), No. 1, 135-163.

[23] K. Scharnhorst, Angles in Complex Vector Spaces, Acta Appl. Math., 69 (2001), No. 1, 95-103.

[24] L. L. Severance, The Theory of Equipollences: Method of Analytical Geometry of Sig. Bellavitis. Meadville, Pa. Tribune 1930.

[25] J. Stewart, Essential Calculus: Early Transcendentals. Belmont, CA. Thomson Higher Education 2007.

[26] V. W. Thurey, The complex Angle in Normed Spaces, Rev. Roumaine Math. Pures Appl., 60 (2015), No. 2, 177-197.

[27] J. E. Valentine and S. G. Wayment, Wilson Angles in Linear Normed Spaces, Pacific Journal of Mathematics, 36(1971), No 1, 239-243. 